\title{
PLANETS AROUND LOW-MASS STARS (PALMS). IV. THE OUTER ARCHITECTURE OF M DWARF PLANETARY SYSTEMS*
}

\author{
Brendan P. Bowler ${ }^{1,2,5,6}$, Michael C. Liu ${ }^{2}$, Evgenya L. Shkolnik ${ }^{3}$, and Motohide Tamura ${ }^{4}$ \\ ${ }^{1}$ California Institute of Technology, Division of Geological and Planetary Sciences, 1200 East California Boulevard, \\ Pasadena, CA 91101, USA; bpbowler@ caltech.edu \\ ${ }^{2}$ Institute for Astronomy, University of Hawai'i, 2680 Woodlawn Drive, Honolulu, HI 96822, USA \\ ${ }^{3}$ Lowell Observatory, 1400 West Mars Hill Road, Flagstaff, AZ 86001, USA \\ ${ }^{4}$ National Astronomical Observatory of Japan, 2-21-1 Osawa, Mitaka, Tokyo 181-8588, Japan \\ Received 2014 July 24; accepted 2014 November 6; published 2014 December 23
}

\begin{abstract}
We present results from a high-contrast adaptive optics imaging search for giant planets and brown dwarfs ( $\gtrsim 1 M_{\text {Jup }}$ ) around 122 newly identified nearby $(\lesssim 40$ pc) young M dwarfs. Half of our targets are younger than $135 \mathrm{Myr}$ and $90 \%$ are younger than the Hyades (620 Myr). After removing 44 close stellar binaries (implying a stellar companion fraction of $>35.4 \% \pm 4.3 \%$ within $100 \mathrm{AU}), 27$ of which are new or spatially resolved for the first time, our remaining sample of 78 single $\mathrm{M}$ dwarfs makes this the largest imaging search for planets around young low-mass stars $\left(0.1-0.6 M_{\odot}\right)$ to date. Our $H$ - and $K$-band coronagraphic observations with Keck/NIRC2 and Subaru/HiCIAO achieve typical contrasts of 12-14 mag and 9-13 mag at $1^{\prime \prime}$, respectively, which correspond to limiting planet masses of $0.5-10 M_{\text {Jup }}$ at 5-33 AU for $85 \%$ of our sample. We discovered four young brown dwarf companions: 1RXS $\mathrm{J} 235133.3+312720 \mathrm{~B}\left(32 \pm 6 M_{\text {Jup }} ; \mathrm{LO}_{-1}^{+2} ; 120 \pm 20 \mathrm{AU}\right), \mathrm{GJ} 3629 \mathrm{~B}\left(64_{-23}^{+30} M_{\text {Jup }} ; \mathrm{M} 7.5 \pm 0.5 ; 6.5 \pm 0.5 \mathrm{AU}\right)$, 1RXS J034231.8+121622 B (35 $\pm 8 M_{\text {Jup }} ; \mathrm{LO} \pm 1 ; 19.8 \pm 0.9$ AU), and 2MASS J15594729+4403595 B (43 \pm $\left.9 M_{\text {Jup }} ; \mathrm{M} 8.0 \pm 0.5 ; 190 \pm 20 \mathrm{AU}\right)$. Over 150 candidate planets were identified; we obtained follow-up imaging for $56 \%$ of these but all are consistent with background stars. Our null detection of planets enables strong statistical constraints on the occurrence rate of long-period giant planets around single $\mathrm{M}$ dwarfs. We infer an upper limit (at the $95 \%$ confidence level) of $10.3 \%$ and $16.0 \%$ for $1-13 M_{\text {Jup }}$ planets between 10-100 AU for hot-start and cold-start (Fortney) evolutionary models, respectively. Fewer than 6.0\% (9.9\%) of M dwarfs harbor massive gas giants in the 5-13 $M_{\text {Jup }}$ range like those orbiting HR 8799 and $\beta$ Pictoris between 10-100 AU for a hot-start (coldstart) formation scenario. The frequency of brown dwarf (13-75 $M_{\mathrm{Jup}}$ ) companions to single $\mathrm{M}$ dwarfs between 10-100 AU is $2.8_{-1.5}^{+2.4} \%$. Altogether we find that giant planets, especially massive ones, are rare in the outskirts of $\mathrm{M}$ dwarf planetary systems. Although the first directly imaged planets were found around massive stars, there is currently no statistical evidence for a trend of giant planet frequency with stellar host mass at large separations as predicted by the disk instability model of giant planet formation.
\end{abstract}

Key words: binaries: visual - brown dwarfs - planetary systems - stars: individual (2MASS J15594729+4403595, GJ 3629, 1RXS J034231.8+121622) - stars: low-mass

Supporting material: machine-readable table

\section{INTRODUCTION}

M dwarfs with masses between $0.1-0.6 M_{\odot}$ constitute the peak of the initial mass function and vastly outnumber all earliertype stars put together. In the solar neighborhood, they make up $\approx 75 \%$ of stars (Henry et al. 2006; Kirkpatrick et al. 2012), which is a good estimate for their galactic-wide rate (Bochanski et al. 2010), and there is some evidence that $\mathrm{M}$ dwarfs represent even larger fractions of stellar populations in evolved galaxies (van Dokkum \& Conroy 2010; Conroy \& van Dokkum 2012). Their abundance and relatively low close binary fractions $(\approx 30 \%$; Fischer \& Marcy 1992; Delfosse et al. 2004; Janson et al. 2012;

\footnotetext{
* Some of the data presented herein were obtained at the W.M. Keck Observatory, which is operated as a scientific partnership among the California Institute of Technology, the University of California and the National Aeronautics and Space Administration. The Observatory was made possible by the generous financial support of the W.M. Keck Foundation. This work was also based on data collected at Subaru Telescope, which is operated by the National Astronomical Observatory of Japan.

5 Caltech Joint Center for Planetary Astronomy Fellow.

6 Visiting Astronomer at the Infrared Telescope Facility, which is operated by the University of Hawaii under Cooperative Agreement No. NNX-08AE38A with the National Aeronautics and Space Administration, Science Mission Directorate, Planetary Astronomy Program.
}

Dieterich et al. 2012) mean that low-mass stars may also be the most common sites of planet formation (Lada 2006).

At small separations ( $\lesssim 2 \mathrm{AU})$ where radial velocity and transit techniques are most sensitive, the frequency of giant planets between $\sim 1-10 M_{\text {Jup }}$ has been found to be relatively low around single $\mathrm{M}$ dwarfs $(2.5 \% \pm 0.9 \%)$ compared to highmass A-type stars (11\% $\pm 2 \%$; Johnson et al. 2010). This wellestablished trend between planet occurrence rate and stellar host mass (Butler et al. 2004, 2006; Endl et al. 2006; Johnson et al. 2007; Lovis \& Mayor 2007; Cumming et al. 2008; Bowler et al. 2010; Bonfils et al. 2013; Gaidos et al. 2013) lends support to the core accretion plus migration model of planet formation (Pollack et al. 1996; Alibert et al. 2005), which predicts fewer gas giants around $\mathrm{M}$ dwarfs as a result of lengthened timescales for planetesimal growth (Laughlin et al. 2004; Ida \& Lin 2005; Kennedy \& Kenyon 2008).

On the other hand, recent radial velocity and transit surveys are showing that Earth- to Neptune-sized planets not only exist in this stellar mass regime (e.g., Udry et al. 2007; Mayor et al. 2009; Charbonneau et al. 2009; Muirhead et al. 2012) but appear to be quite common (Bonfils et al. 2013; Berta et al. 2013). In particular, Swift et al. (2013) and Dressing \& 
Charbonneau (2013) find that the average rate of small planets from Kepler is about one per star for periods shorter than 50 days, implying a vast galaxy-wide presence of rocky planets (Morton \& Swift 2014). This in turn has generated increasing interest in the habitability of planets around $M$ dwarfs since the nearest examples of habitable Earths may orbit low-mass stars (e.g., Joshi et al. 1997; Cantrell et al. 2013; Quintana et al. 2014).

Far less is known about planets at moderate separations of 2-10 AU. Although microlensing probes the full range of planetary masses in this region (Gould \& Loeb 1992), the masses and metallicities of the host stars are usually poorly constrained with this technique and so are of limited value for statistical constraints. The lensing signal from the star itself becomes very weak beyond projected separations of $\sim 10$ AU (Han 2006, 2009), leading to an ambiguity between isolated planetary-mass objects and bound planets on wide orbits (Sumi et al. 2011). Nevertheless, initial statistical results point to a large reservoir of planets orbiting $\mathrm{M}$ dwarfs at moderate separations. Gould et al. (2010) find that the frequency of planets in the ice giant to gas giant range ( $\gtrsim 0.05 M_{\text {Jup }}$ ) is a factor of 8 time larger than those from Doppler studies at small separations. In a follow-up study, Cassan et al. (2012) measure a frequency of $17_{-9}^{+6} \%\left(52_{-29}^{+22} \%\right)$ for 0.3-10 $M_{\text {Jup }}\left(10-30 M_{\oplus}\right)$ planets between 0.5-10 AU. Across the entire range of sensitivity $\left(10 M_{\oplus}-10 M_{\mathrm{Jup}}, 0.5-10 \mathrm{AU}\right)$, these occurrence rates imply that $\mathrm{M}$ dwarfs harbor on average $1.6_{-0.9}^{+0.7}$ planets per star. This result was recently bolstered by Clanton \& Gaudi (2014), who found that the total number of $1-10^{4} M_{\oplus}$ planets with periods of $1-10^{4}$ days is $1.9 \pm$ 0.5 by combining statistical results from radial velocity and microlensing surveys.

Another form of planet population statistical analysis in this intermediate-separation regime comes from combining longbaseline radial velocity monitoring with adaptive optics (AO) imaging. Montet et al. (2014) apply this method to old M dwarfs in the field and find a frequency of $6.5 \% \pm 3 \%$ for $1-13 M_{\mathrm{Jup}}$ planets within $20 \mathrm{AU}$, which is consistent with microlensing results over the same region.

Beyond $\sim 10 \mathrm{AU}$, direct imaging is the best way to study the outer architecture of planetary systems. Following the discoveries of planets orbiting the A-type stars HR 8799, Fomalhaut, and $\beta$ Pic (Marois et al. 2008, 2010b; Kalas et al. 2008; Lagrange et al. 2010), high-mass stars have received the most attention in direct imaging planet searches (Ehrenreich et al. 2010; Janson et al. 2011; Vigan et al. 2012; Rameau et al. 2013a; Nielsen et al. 2013). Yet despite their prevalence in the galaxy, imaging surveys have mostly neglected low-mass stars, so little is known about the demographics of gas giants on wide orbits around $\mathrm{M}$ dwarfs. This is largely due to a dearth of known nearby young $\mathrm{M}$ dwarfs, a population that has been substantially enlarged over the past few years (Shkolnik et al. 2009, 2012; Schlieder et al. 2012c; Malo et al. 2013, 2014a; Rodriguez et al. 2013). Low-mass stars are also optically faint and typically result in poorer AO performance than their brighter, earliertype counterparts. Furthermore, few of the surveys that have incorporated $\mathbf{M}$ dwarfs expressly vetted close binaries from their statistical analyses, which is crucial if the results are to be compared with radial velocity planet searches of single stars. A handful of surveys sensitive to 1-10 $M_{\text {Jup }}$ companions have targeted single, young, M0-M5 stars: Biller et al. (2007) observed 12 targets with Very Large Telescope (VLT)/MMT Simultaneous Differential Imaging (SDI), Lafrenière et al. (2007a) imaged 16 stars with Gemini-North/NIRI, Chauvin et al.
(2010) imaged 16 single M dwarfs with VLT/NaCo, Delorme et al. (2012) targeted 12 stars with VLT/NaCo in $L^{\prime}$ band, and Biller et al. (2013) observed 35 single $\mathrm{M}$ dwarfs with GeminiSouth/NICI. ${ }^{7}$

The aim of the Planets Around Low-Mass Stars (PALMS) survey is to find young giant planets and brown dwarfs for spectroscopic characterization and to measure the frequency of gas giants orbiting $\mathrm{M}$ dwarfs beyond $10 \mathrm{AU}$. In Bowler et al. (2012b) and Bowler et al. (2012a), we discovered two new brown dwarf companions to young $M$ dwarfs in our sample. ${ }^{8}$ In this paper, we present two additional substellar companion discoveries and the statistical analysis of our entire sample. Below we describe our target selection, observations, processing pipeline, discoveries, survey statistical analysis, and implications for giant planet formation around low-mass stars.

\section{TARGET SELECTION}

Our targets are selected primarily for their youth and proximity in order to achieve the highest sensitivity to giant planets at small separations. Previously known visual binaries with physical separations $\lesssim 100$ AU have been excluded since moderateseparation ( 5-100 AU) binaries disperse protoplanetary disks on rapid timescales (Duchêne 2010; Kraus et al. 2012), limiting the raw ingredients of planet formation and diminishing the region of dynamically stable orbits in these systems. In addition, we have specifically designed our survey to compare with statistical results from radial velocity programs, which generally discard close binaries from their samples. We have also prioritized targets not previously observed in direct imaging surveys to minimize target selection biases and increase the chances of new discoveries.

Among our 122 targets, 69 originate from a recent search for nearby young M dwarfs by Shkolnik et al. (2009) and Shkolnik et al. (2012). Motivated by the dearth of known lowmass members of young moving groups (YMGs), Shkolnik et al. (2009) identified 144 X-ray active $M$ dwarfs with distances $\lesssim 30$ pc and ages of $\sim 10-300$ Myr. In a follow-up study, Shkolnik et al. (2012) obtained parallaxes for about half of these systems and found several dozen probable kinematic members of YMCs. Targets from Shkolnik et al. (2009) have been vetted for close spectroscopic binaries with few-day periods (Shkolnik et al. 2010), which also produce activity as a result of rotationally enhanced dynamo activity caused by tidal locking (e.g., Torres et al. 2002; Kraus et al. 2011).

Another 42 targets in our sample are drawn from an ongoing, complementary search for nearby young M dwarfs using Galaxy Evolution Explorer (GALEX) data (Shkolnik et al. 2011; E. L. Shkolnik et al., in preparation). Among these, 10 systems have been kinematically tied to YMGs by Malo et al. (2013), Lépine \& Simon (2009), and Riedel et al. (2014) and two new candidate members are identified in this work (LHS 2613 and NLTT 48651). Seven other systems (LHS1864 AB, NLTT 26359, LHS 2672, G 202-48, GJ 3997 AB; LP 447-38 AB; LHS 3321) either show $\mathrm{H} \alpha$ absorption or have red $N U V-W 1$

\footnotetext{
7 Other imaging programs that have also observed single young M dwarfs with ground-based adaptive optics or the Hubble Space Telescope have primarily been sensitive to brown dwarfs at wide separations, rarely reaching 1-5 $M_{\text {Jup }}$ limits at small separations of $\sim 10$ AU (McCarthy \& Zuckerman 2004; Lowrance et al. 2005; Daemgen et al. 2007; Allen \& Reid 2008). 8 As part of a complementary imaging survey targeting a much larger sample of young $\mathrm{M}$ dwarfs with shorter exposures, we have also discovered the young L-type companion 2MASS J01225093-2439505 B which has a mass at the deuterium-burning limit (Bowler et al. 2013).
} 

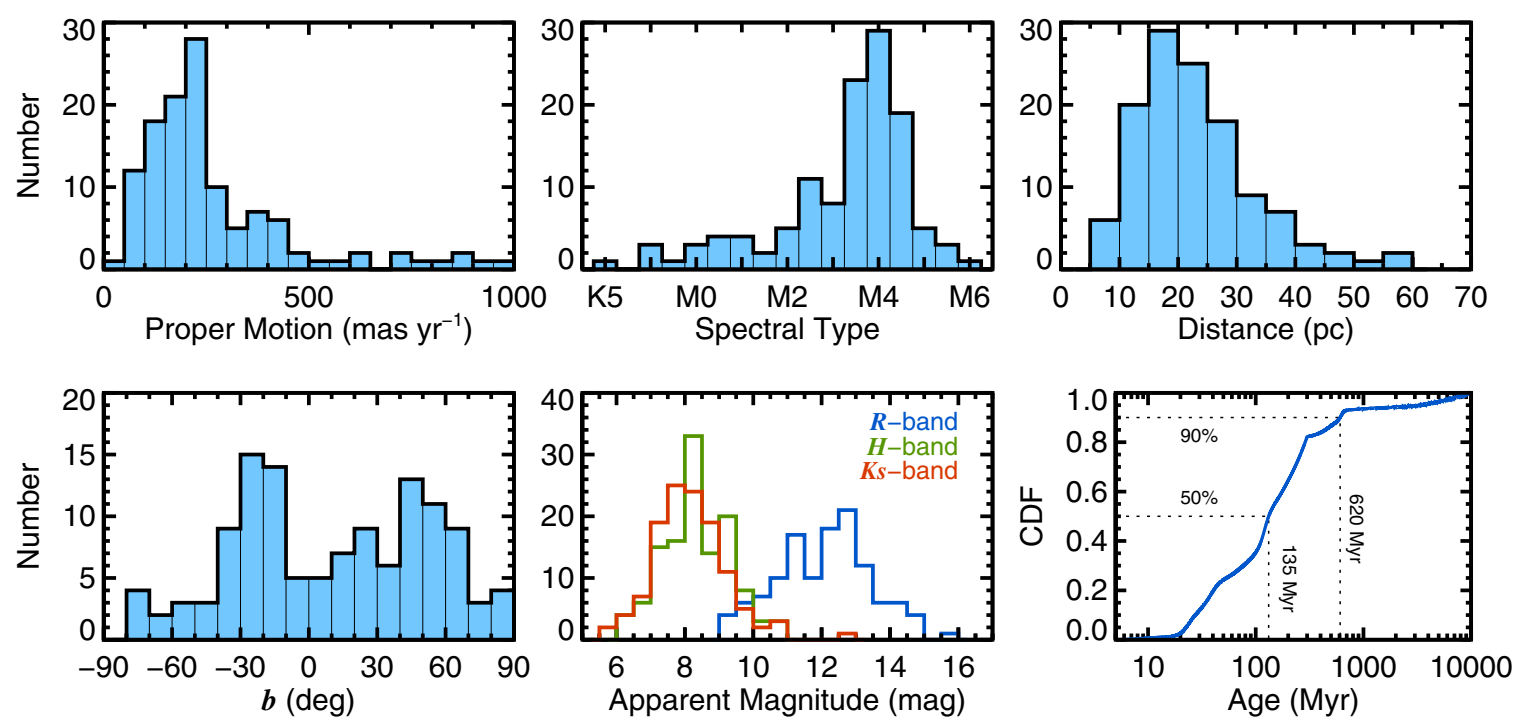

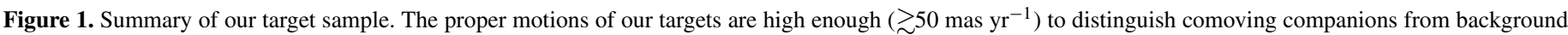

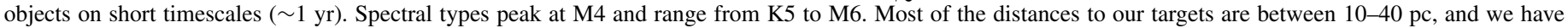

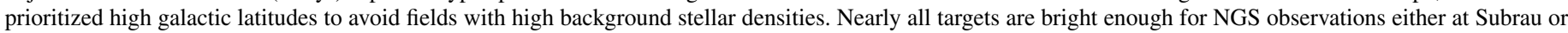

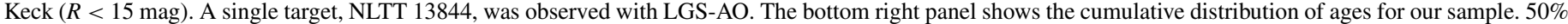
are younger than $135 \mathrm{Myr}$ and $90 \%$ of our targets are younger than $620 \mathrm{Myr}$.

colors (>13 mag) compared to YMG members (Rodriguez et al. 2013). These targets appear to be old inactive field stars that passed early $N U V$ selection cuts, so we adopt minimum ages from the activity-lifetime relations of West et al. (2008). One system, 2MASS J04220833-2849053 AB, has its age constrained from the detection of Li I $\lambda 6708$ absorption by Torres et al. (2006; see Appendix C). Similarly, the age of 2MASS J15594729+4403595 is constrained from signatures of low gravity in the spectrum of its substellar companion (Section 5.1.4). The remaining 21 of these 42 targets show photometric and spectroscopic indications of youth similar to known YMG members. A detailed analysis of their ages, including a discussion of high-resolution optical spectroscopy for these targets, will be presented in a forthcoming paper (E. L. Shkolnik et al., in preparation). For this work we adopt conservative age ranges of 10-300 Myr similar to Shkolnik et al. (2009) for the 21 targets without age estimates in the literature.

Finally, 11 targets are compiled from the literature from recent searches for $\mathrm{M}$ dwarf members of YMGs. Six originate from Schlieder et al. (2012a, 2012b, 2012c), while another five are from Looper et al. (2010b, TWA 30A), Riedel et al. (2011, AP Col), Scholz et al. (2005a, L 449-1 AB), Lépine \& Simon (2009, TYC 7443-1102-1), and Lowrance et al. (2005, GJ 354.1 B).

Where available, age estimates and YMG memberships have been taken from the literature. Altogether, 46 targets (38\% of our total sample) are associated with YMGs. Ages for YMG members (or likely members) are listed in Table $1 . .^{9}$ Five systems are kinematically linked to YMGs here for the first time: LHS 2613 (Argus), 1RXS J022735.8+471021 (AB Dor),

\footnotetext{
9 Recently the ages of several of the youngest moving groups have been called into question from Li depletion boundary measurements. For example, Binks \& Jeffries (2014) find an older age of $21 \pm 4$ for the $\beta$ Pic YMG from its Li-depletion boundary compared to its isochronal age of $\approx 12 \mathrm{Myr}$. These results are bolstered by recent studies by Malo et al. (2014b) and Mamajek \& Bell (2014). Similarly, Kraus et al. (2014) infer a Li-depletion age of $\approx 40 \mathrm{Myr}$ for the Tuc-Hor moving group, which is roughly 10 Myr older than its age from isochrone fitting. Here we adopt the more recently determined and internally consistent ages of $23 \pm 3$ Myr for the $\beta$ Pic MG and $35 \pm 5$ Myr for Tuc-Hor.
}

Table 1

Adopted Ages for Young Moving Group Members

\begin{tabular}{lccc}
\hline \hline Moving Group & No. of Targets & Age & Age Ref \\
\hline TWA & 1 & $8 \pm 2 \mathrm{Myr}$ & $1,2,3,4,5$ \\
$\beta$ Pic & 8 & $23 \pm 3 \mathrm{Myr}$ & $6,7,8,9$ \\
Carina, Columba & 5 & $30 \pm 5 \mathrm{Myr}$ & 10 \\
Tuc-Hor & 3 & $35 \pm 5 \mathrm{Myr}$ & $10,11,12,13$ \\
Argus & 6 & $40 \pm 5 \mathrm{Myr}$ & $10,14,15$ \\
AB Doradus & 10 & $120 \pm 10 \mathrm{Myr}$ & $16,17,18,19$ \\
Castor & 3 & $400 \pm 100 \mathrm{Myr}$ & $20,21,22,23$ \\
Ursa Major & 6 & $500 \pm 100 \mathrm{Myr}$ & $24,25,26$ \\
Hyades & 4 & $620 \pm 30 \mathrm{Myr}$ & $27,28,29,30$ \\
\end{tabular}

References. (1) Webb et al. 1999; (2) Navascués 2006; (3) Mamajek 2005; (4) Torres et al. 2006; (5) Weinberger et al. 2013; (6) Yee \& Jensen 2010; (7) Binks \& Jeffries 2014; (8) Malo et al. 2014b; (9) Mamajek \& Bell 2014; (10) Torres et al. 2008; (11) Torres et al. 2000; (12) Zuckerman et al. 2001; (13) Kraus et al. 2014; (14) Torres et al. 2003; (15) Silva et al. 2013; (16) Zuckerman et al. 2004; (17) Ortega et al. 2007; (18) Luhman et al. 2005; (19) Barenfeld et al. 2013; (20) Barrado y Navascués 1998; (21) Torres \& Ribas 2002; (22) Ribas 2003; (23) Mamajek 2012; (24) Eggen 1983; (25) Soderblom \& Mayor 1993; (26) King et al. 2003; (27) Perryman et al. 1998; (28) Eggen 1998; (29) Lebreton et al. 2001; (30) Degennaro et al. 2009.

\section{NLTT 48651 (AB Dor), GJ 354.1 B (Carina), and G 227-22} (UMa).

Figure 1 and Table 2 summarize the properties of our sample. Proper motions mostly originate from the UCAC4 database (Zacharias et al. 2013) and generally fall between 100-500 mas $\mathrm{yr}^{-1}$, which is high enough so that background stars can be distinguished from bona fide comoving companions on timescales of about one year. Spectral types are compiled from the literature and range from K5 to M6 $\left(\approx 0.2-0.6 M_{\odot}\right)$, with most of the sample falling between M3 and M5. 69 targets (57\% of the sample) have parallactic distances. For the rest, we have either adopted photometric distances (42 targets) or kinematic distances based on YMG memberships (11 targets) from the literature (see Table 2 for details). 97 targets (80\% of 
Table 2

Target Sample

\begin{tabular}{|c|c|c|c|c|c|c|c|c|c|c|c|c|c|}
\hline Name & $\alpha_{\mathrm{J} 2000}$ & $\delta_{\mathrm{J} 2000}$ & $\begin{array}{c}\mu_{\alpha} \cos \delta \\
\left(\operatorname{mas~yr}^{-1}\right)\end{array}$ & $\begin{array}{c}\mu_{\delta} \\
\left(\operatorname{mas~yr}^{-1}\right)\end{array}$ & PM Ref & $\begin{array}{c}\text { SpT } \\
( \pm 0.5)\end{array}$ & SpT Ref & $\begin{array}{l}\text { Distance } \\
\text { (pc) }\end{array}$ & Method & Dist Ref & $\mathrm{YMG}^{\mathrm{a}}$ & $\begin{array}{l}\text { Age } \\
(\mathrm{Myr})\end{array}$ & Age Ref \\
\hline G 217-32 AB & 000742.64 & 602254.3 & $342.0 \pm 8.0$ & $-29.0 \pm 8.0$ & $\mathrm{Z} 13$ & M4.5 & L13 & $14.6 \pm 0.4$ & $\pi$ & D14 & & $35-300$ & Sh09 \\
\hline 1RXS J001557.5-163659 AB & 001558.08 & -163657.9 & $-110.2 \pm 2.2$ & $35.0 \pm 2.2$ & $\mathrm{Z} 13$ & M4.0 & Ri06 & $18.0 \pm 0.4$ & $\pi$ & Sh12 & & $35-300$ & Sh09 \\
\hline GJ $3030 \mathrm{AB}$ & 002157.81 & 491238.0 & $221.4 \pm 10.8$ & $-32.7 \pm 12.6$ & $\mathrm{Z} 13$ & M2.4 & Sh09 & $25 \pm 9$ & Phot & Sh12 & Carina? & $30 \pm 5$ & Sh12 \\
\hline NLTT 1875 & 003504.88 & 595308.0 & $223.4 \pm 2.1$ & $-3.4 \pm 1.5$ & $\mathrm{Z} 13$ & M4.3 & Sh09 & $38.3 \pm 2.2$ & $\pi$ & D14 & & $100-500$ & B14 \\
\hline G $132-50 \mathrm{Aab}$ & 010340.14 & 405128.9 & $126.7 \pm 0.7$ & $-167.2 \pm 0.8$ & $\mathrm{Z} 13$ & M0.0 & $\mathrm{L} 13$ & $29.9 \pm 2.2$ & $\pi$ & vL07 & AB Dor & $120 \pm 10$ & Sh12 \\
\hline G 172-56 & 012912.57 & 481935.5 & $214.0 \pm 8.0$ & $-22.0 \pm 8.0$ & $\mathrm{Z} 13$ & M5.4 & S09 & $18 \pm 3$ & Phot & Sh12 & $\ldots$ & $60-300$ & Sh09 \\
\hline G 272-43 & 013358.00 & -173823.5 & $44.4 \pm 2.1$ & $-185.1 \pm 3.5$ & $\mathrm{Z} 13$ & M3.5 & Ri06 & $16 \pm 7$ & Phot & L11 & & $10-300$ & Sh15 \\
\hline G 271-110 & 013655.30 & -064736.4 & $172.6 \pm 8.0$ & $-84.2 \pm 8.0$ & $\mathrm{Z} 13$ & M4.0 & Ri06 & $24.0 \pm 0.4^{\mathrm{b}}$ & $\pi$ & Sh12 & $\beta$ Pic & $23 \pm 3$ & M13 \\
\hline TYC 1752-63-1 & 013723.23 & 265712.0 & $120.2 \pm 0.9$ & $-126.5 \pm 1.4$ & $\mathrm{Z} 13$ & K7 & Sc10 & $37 \pm 1$ & Kin & M13 & AB Dor? & $120 \pm 10$ & Sc 10 \\
\hline G 272-115 & 015311.33 & -210543.3 & $271.4 \pm 8.0$ & $72.5 \pm 8.0$ & $\mathrm{Z} 13$ & M1.5 & Ri06 & $16 \pm 7$ & Phot & L11 & & $10-300$ & Sh15 \\
\hline NLTT 6549 AB & 015813.61 & 484419.7 & $213.0 \pm 8.0$ & $-41.0 \pm 8.0$ & $\mathrm{Z} 13$ & M1.5 & Sh09 & $44 \pm 18$ & Phot & Sh12 & Hyades? & $620 \pm 30$ & Sh12 \\
\hline GJ 3136 & 020853.60 & 492656.6 & $231.9 \pm 10.8$ & $-284.5 \pm 12.5$ & $\mathrm{Z} 13$ & M4.0 & L13 & $15 \pm 6$ & Phot & Sh12 & AB Dor? & $120 \pm 10$ & Sh12 \\
\hline LP $353-51$ & 022326.64 & 224406.9 & $96.1 \pm 2.7$ & $-114.2 \pm 1.0$ & $\mathrm{Z} 13$ & M0.5 & L13 & $28.7 \pm 2.5$ & $\pi$ & vL07 & $\beta$ Pic & $23 \pm 3$ & Sc10 \\
\hline 1RXS J022735.8+471021 & 022737.26 & 471004.5 & $119.0 \pm 8.0$ & $-183.0 \pm 8.0$ & $\mathrm{Z} 13$ & M4.6 & Sh09 & $27.4 \pm 1.7$ & $\pi$ & D14 & AB Dor & $120 \pm 10$ & B14 \\
\hline 1RXS J023138.7+445640 & 023139.27 & 445638.8 & $94.4 \pm 5.4$ & $-47.3 \pm 3.7$ & $\mathrm{Z} 13$ & M4.4 & Sh09 & $16 \pm 4$ & Phot & Sh12 & & $40-300$ & Sh09 \\
\hline G 75-35 & 024115.11 & -043217.7 & $352.3 \pm 8.0$ & $-58.8 \pm 8.0$ & $\mathrm{Z} 13$ & M4.0 & Ri06 & $16 \pm 7$ & Phot & L11 & $\ldots$ & $10-300$ & Sh15 \\
\hline 2MASS J03033668-2535329 AB & 030336.69 & -253532.9 & $213.2 \pm 8.0$ & $94.5 \pm 8.0$ & $\mathrm{Z} 13$ & M0.0 & Ri06 & $38.6 \pm 4.2$ & $\pi$ & vL07 & $\ldots$ & $10-300$ & Sh15 \\
\hline LP $247-13$ & 031537.83 & 372414.3 & $209.0 \pm 8.0$ & $-97.0 \pm 8.0$ & $\mathrm{Z} 13$ & M2.7 & Sh09 & $34.4 \pm 0.6$ & $\pi$ & Sh12 & Hyades & $620 \pm 30$ & R11, Sh12 \\
\hline 1RXS J034231.8+121622 AB & 034231.80 & 121622.6 & $196.8 \pm 2.2$ & $-16.3 \pm 3.8$ & $\mathrm{Z} 13$ & M4.0 & Ri06 & $23.9 \pm 1.1$ & $\pi$ & D14 & $\ldots$ & $60-300$ & S09 \\
\hline G $160-54$ ABC & 041345.86 & -050904.9 & $182.0 \pm 8.0$ & $-112.5 \pm 8.0$ & $\mathrm{Z} 13$ & {$[\mathrm{M} 4]^{\mathrm{c}}$} & L11 & $21 \pm 9$ & Phot & $\mathrm{L} 11$ & $\ldots$ & $10-300$ & Sh15 \\
\hline 2MASS J04220833-2849053 AB & 042208.33 & -284905.4 & $-80.6 \pm 1.3$ & $-86.2 \pm 1.9$ & $\mathrm{Z} 13$ & K7.0 & Ri06 & $28 \pm 12$ & Phot & L11 & $\ldots$ & $50-200$ & B14, Sh15 \\
\hline GJ 3287 & 042741.30 & 593516.7 & $119.0 \pm 8.0$ & $-200.0 \pm 8.0$ & $\mathrm{Z} 13$ & M3.8 & Sh09 & $22.5 \pm 1.2$ & $\pi$ & D14 & $\ldots$ & $35-300$ & Sh09 \\
\hline LP 834-32 & 043536.19 & -252734.7 & $82.8 \pm 10.3$ & $-179.7 \pm 12.4$ & $\mathrm{Z} 13$ & M3.5 & Ri06 & $15 \pm 1$ & Kin & M13 & AB Dor? & $120 \pm 10$ & M13 \\
\hline NLTT 13844 & 044505.62 & 432434.2 & $388.0 \pm 8.0$ & $-638.0 \pm 8.0$ & $\mathrm{Z} 13$ & M4.6 & Sh09 & $21.0 \pm 0.5$ & $\pi$ & D14 & $\ldots$ & $60-300$ & Sh09 \\
\hline 2MASS J04472312-2750358 & 044723.13 & -275035.8 & $82.0 \pm 15.9$ & $156.0 \pm 16.7$ & $\mathrm{Z} 13$ & $\mathrm{~K} 7.0$ & Ri06 & $20 \pm 3$ & Phot & Sh12 & $\ldots$ & $400-1200$ & Sh09 \\
\hline G 81-34 AB & 044929.47 & 482845.9 & $180.0 \pm 8.0$ & $-195.0 \pm 8.0$ & $\mathrm{Z} 13$ & M4.0 & Sh09 & $21.2 \pm 0.9$ & $\pi$ & D14 & & $35-300$ & Sh09 \\
\hline 1RXS J045101.0+312734 & 045101.38 & 312723.9 & $198.0 \pm 8.0$ & $-41.0 \pm 8.0$ & $\mathrm{Z} 13$ & M3.7 & Sh09 & $13 \pm 4$ & Phot & Sh12 & & $35-300$ & Sh09 \\
\hline L $449-1 \mathrm{AB}$ & 051722.93 & -352154.5 & $-234.2 \pm 12.1$ & $-162.6 \pm 9.5$ & Ri14 & M4.0 & Ri06 & $11.85 \pm 0.19$ & $\pi$ & Ri14 & UMa? & $500 \pm 100$ & Ri14 \\
\hline 1RXS J055446.0+105559 & 055445.74 & 105557.1 & $-134.0 \pm 8.0$ & $-67.0 \pm 8.0$ & $\mathrm{Z} 13$ & M3.0 & $\mathrm{L} 13$ & $25.4 \pm 1.9$ & $\pi$ & Sh12 & $\ldots$ & $20-150$ & Sh09 \\
\hline GJ $3371 \mathrm{~A}$ & 055937.75 & 583535.1 & $15.7 \pm 2.1$ & $-256.5 \pm 1.9$ & $\mathrm{Z} 13$ & M1.0 & L13 & $13.5 \pm 0.3$ & $\pi$ & vL07 & Castor? & $400 \pm 100$ & Sh12 \\
\hline GJ 3372 B & 055955.69 & 583415.6 & $5.9 \pm 2.8$ & $-255.3 \pm 3.7$ & $\mathrm{Z} 13$ & M4.2 & Sh09 & $13.5 \pm 0.3^{\mathrm{b}}$ & $\pi$ & vL07 & Castor? & $400 \pm 100$ & Sh12 \\
\hline AP Col & 060452.16 & -343336.1 & $21.4 \pm 12.1$ & $341.2 \pm 9.4$ & Ri14 & M5.0 & Ri06 & $8.39 \pm 0.07$ & $\pi$ & Ri11 & Argus & $40 \pm 5$ & Ri11 \\
\hline G $249-36$ & 060529.36 & 604923.2 & $288.8 \pm 5.0$ & $-787.2 \pm 5.0$ & Ro10 & M4.9 & Sh09 & $14.0 \pm 0.4$ & $\pi$ & D14 & & $60-300$ & Sh09 \\
\hline 2MASS J06131330-2742054 AB & 061313.31 & -274205.5 & $-13.1 \pm 1.6$ & $-0.3 \pm 1.3$ & $\mathrm{Z} 13$ & M3.5 & Ri06 & $29.4 \pm 0.9$ & $\pi$ & Ri14 & $\beta$ Pic & $23 \pm 3$ & M13, Ri14 \\
\hline 2MASS J06180730+7506032 & 061807.30 & 750603.3 & $-41.9 \pm 1.1$ & $68.7 \pm 2.4$ & $\mathrm{Z} 13$ & M2.0 & L13 & $18 \pm 8$ & Phot & L11 & $\ldots$ & $10-300$ & Sh15 \\
\hline GJ 3395 & 063101.16 & 500248.6 & $-110.3 \pm 1.0$ & $-170.2 \pm 0.6$ & $\mathrm{Z} 13$ & M1.0 & L13 & $28 \pm 9$ & Phot & Sh12 & $\ldots$ & $20-150$ & Sh09 \\
\hline LHS $1864 \mathrm{AB}$ & 064349.70 & 510821.0 & $91.0 \pm 5.1$ & $-881.7 \pm 5.1$ & Ro10 & M3.5 & L13 & $19.1 \pm 1.7$ & $\pi$ & vA95 & $\ldots$ & $3-10 \times 10^{3 d}$ & B14, Sh15 \\
\hline G 108-36 & 065159.01 & 031255.3 & $-213.0 \pm 8.0$ & $-46.0 \pm 8.0$ & $\mathrm{Z} 13$ & M2.5 & Sh09 & $22.2 \pm 0.6$ & $\pi$ & Sh12 & $\ldots$ & $20-150$ & Sh09 \\
\hline 1RXS J073829.3+240014 & 073829.52 & 240008.8 & $-179.0 \pm 8.0$ & $-107.0 \pm 8.0$ & $\mathrm{Z} 13$ & M3.5 & L13 & $18.9 \pm 0.9$ & $\pi$ & Sh12 & $\ldots$ & $20-300$ & Sh09 \\
\hline 1RXS J091744.5+461229 AB & 091744.73 & 461224.7 & $-129.1 \pm 0.7$ & $-19.3 \pm 1.2$ & Z13 & M2.5 & L13 & $32 \pm 9$ & Phot & Sh12 & & $20-150$ & Sh09 \\
\hline GJ $354.1 \mathrm{~B}$ & 093248.27 & 265944.3 & $-134.0 \pm 8.0$ & $-242.0 \pm 8.0$ & $\mathrm{Z} 13$ & M5.5 & L05 & $17.8 \pm 0.2^{\mathrm{b}}$ & $\pi$ & vL07 & Carina? & $30 \pm 5$ & B14, Sh15 \\
\hline PYC J09362+3731 AB & 093615.93 & 373145.7 & $-102.7 \pm 0.7$ & $-89.6 \pm 0.7$ & $\mathrm{Z} 13$ & M0.5 & L13 & $33.7 \pm 2.8$ & $\pi$ & vL07 & $\ldots$ & $10-10^{4}$ & $\mathrm{Sc} 12 \mathrm{c}$ \\
\hline G $161-71$ & 094454.22 & -122054.4 & $-331.1 \pm 8.0$ & $30.9 \pm 8.0$ & $\mathrm{Z} 13$ & M5.0 & Ri06 & $8 \pm 3$ & Phot & L11 & Argus? & $40 \pm 5$ & M13 \\
\hline NLTT $22741 \mathrm{~A}$ & 095104.60 & 355809.8 & $-100.8 \pm 4.3$ & $-154.7 \pm 4.3$ & Z13 & M4.5 & Re06 & $29.8 \pm 1.7$ & $\pi$ & D14 & $\ldots$ & $100-200$ & RW06 \\
\hline GJ 3577 A & 095918.80 & 435025.6 & $-104.0 \pm 8.0$ & $-222.0 \pm 8.0$ & Ro10 & M3.3 & H97 & $24.9 \pm 1.1$ & $\pi$ & D14 & $\ldots$ & $25-300$ & Sh09 \\
\hline GJ $3578 \mathrm{~B}$ & 095920.94 & 435025.9 & $-101.1 \pm 4.8$ & $-224.3 \pm 4.8$ & $\mathrm{Z} 13$ & M3.8 & H97 & $24.9 \pm 1.1^{\mathrm{b}}$ & $\pi$ & D14 & $\ldots$ & $25-300^{\mathrm{e}}$ & Sh09 \\
\hline
\end{tabular}


Table 2

(Continued)

\begin{tabular}{|c|c|c|c|c|c|c|c|c|c|c|c|c|c|}
\hline Name & $\alpha_{\mathrm{J} 2000}$ & $\delta_{\mathrm{J} 2000}$ & $\begin{array}{c}\mu_{\alpha} \cos \delta \\
\left(\operatorname{mas~yr}^{-1}\right)\end{array}$ & $\begin{array}{c}\mu_{\delta} \\
\left(\operatorname{mas~yr}^{-1}\right)\end{array}$ & PM Ref & $\begin{array}{c}\text { SpT } \\
( \pm 0.5)\end{array}$ & SpT Ref & $\begin{array}{l}\text { Distance } \\
\quad(\mathrm{pc})\end{array}$ & Method & Dist Ref & $\mathrm{YMG}^{\mathrm{a}}$ & $\begin{array}{l}\text { Age } \\
(\mathrm{Myr})\end{array}$ & Age Ref \\
\hline G 196-3 A & 100421.49 & 502313.6 & $-137.6 \pm 3.0$ & $-199.3 \pm 5.1$ & Z13 & M2.5 & L13 & $24.4 \pm 2.5$ & $\pi$ & ZO14 & & $10-150$ & K08, A13 \\
\hline GJ $2079 \mathrm{AB}$ & 101419.19 & 210429.8 & $-137.2 \pm 0.5$ & $-165.8 \pm 0.5$ & Z13 & M0.5 & L13 & $23.1 \pm 1.0$ & $\pi$ & vL07 & Carina? & $30 \pm 5$ & Sh12 \\
\hline GJ $3629 \mathrm{AB}$ & 105120.60 & 360725.6 & $-192.0 \pm 1.0$ & $-48.0 \pm 6.0$ & $\mathrm{Z} 05$ & M3.0 & H97 & $32.3 \pm 2.4$ & $\pi$ & D14 & $\ldots$ & $25-300$ & Sh09 \\
\hline PYC J10571+0544 AB & 105711.39 & 054454.7 & $-55.3 \pm 1.9$ & $-40.4 \pm 2.5$ & $\mathrm{Z} 13$ & M2.0 & Sc12a & $46 \pm 11$ & Phot & L11 & $\beta$ Pic? & $23 \pm 3$ & Sc12a \\
\hline GJ 3639 & 110310.00 & 363908.5 & $-196.0 \pm 8.0$ & $27.0 \pm 8.0$ & $\mathrm{Z} 13$ & M3.5 & H97 & $24.0 \pm 0.9$ & $\pi$ & D14 & $\ldots$ & $25-300$ & Sh09 \\
\hline NLTT 26114 & 110321.25 & 133757.1 & $-185.0 \pm 8.0$ & $68.0 \pm 8.0$ & $\mathrm{Z} 13$ & M4.0 & L13 & $15.4 \pm 0.7$ & $\pi$ & Sh12 & $\ldots$ & $25-300$ & Sh09 \\
\hline NLTT 26359 & 110727.73 & -19 1729.4 & $-238.6 \pm 2.4$ & $-48.7 \pm 2.1$ & $\mathrm{Z} 13$ & $\mathrm{~K} 5^{\mathrm{f}}$ & S86 & $18.7 \pm 0.6$ & $\pi$ & vL07 & $\ldots$ & $10-300$ & Sh15 \\
\hline G 119-62 & 111152.15 & 333211.1 & $-176.6 \pm 6.6$ & $127.1 \pm 6.1$ & Z13 & M4.0 & L13 & $14.6 \pm 2.7$ & $\pi$ & vA95 & $\ldots$ & $25-300$ & Sh09 \\
\hline 2MASS J11240434+3808108 & 112404.35 & 380810.9 & $121.7 \pm 2.6$ & $-12.4 \pm 2.3$ & Z13 & M4.5 & $\operatorname{Re} 07$ & $20.3 \pm 1.3^{\mathrm{g}}$ & Phot & B14 & UMa? & $500 \pm 100$ & Sh12 \\
\hline TWA $30 \mathrm{~A}$ & 113218.32 & -301951.8 & $-87.8 \pm 1.3$ & $-25.2 \pm 1.3$ & $\mathrm{Z} 13$ & $M 5^{\mathrm{f}}$ & Lo10 & $42 \pm 2$ & Kin & Lo10 & TWA & $8 \pm 2$ & Lo10 \\
\hline G $10-52$ & 114835.49 & 074140.4 & $137.0 \pm 8.0$ & $-164.0 \pm 8.0$ & $\mathrm{Z} 13$ & $\mathrm{M} 3.5^{\mathrm{f}}$ & Sh09 & $20.7 \pm 0.9$ & $\pi$ & Sh12 & $\ldots$ & $25-300$ & Sh09 \\
\hline 2MASS J12062214-1314559 AB & 120622.14 & -131456.0 & $73.0 \pm 2.4$ & $-61.3 \pm 1.3$ & $\mathrm{Z} 13$ & M3.5 & Ri06 & $27 \pm 10$ & Phot & $\mathrm{J} 12$ & $\ldots$ & $10-300$ & Sh15 \\
\hline LP 734-34 & 121028.34 & -131023.5 & $242.8 \pm 8.0$ & $-342.7 \pm 8.0$ & Z13 & M4.5 & Ri06 & $16 \pm 4$ & Phot & L11 & $\ldots$ & $10-300$ & Sh15 \\
\hline G 13-33 AB & 122250.62 & -040446.2 & $-255.0 \pm 8.0$ & $-65.7 \pm 8.0$ & Z13 & M4.5 & S05b & $15 \pm 3$ & Phot & Sh12 & $\ldots$ & $10-300$ & Sh15 \\
\hline LP 735-48 AB & 122644.13 & -122917.5 & $-164.4 \pm 1.2$ & $-82.7 \pm 1.2$ & $\mathrm{Z} 13$ & $\mathrm{M}^{\mathrm{h}}$ & B14 & $24 \pm 8^{\mathrm{h}}$ & Phot & B14 & $\ldots$ & $10-300$ & Sh15 \\
\hline GJ 3729 AB & 122902.90 & 414349.7 & $-196.0 \pm 8.0$ & $-222.0 \pm 8.0$ & $\mathrm{Z} 13$ & M4.0 & L13 & $17 \pm 5$ & Phot & Sh12 & Tuc-Hor? & $35 \pm 5$ & Sh12 \\
\hline 2MASS J12383713-2703348 AB & 123837.13 & -270334.9 & $-185.1 \pm 5.1$ & $-185.2 \pm 5.1$ & Ro10 & M2.5 & Ri06 & $25 \pm 1$ & Kin & M13 & AB Dor? & $120 \pm 10$ & M13 \\
\hline 1RXS J124147.5+564506 & 124147.37 & 564513.8 & $111.8 \pm 1.6$ & $0.6 \pm 1.5$ & $\mathrm{Z13}$ & M3.0 & Ri06 & $30 \pm 11$ & Phot & Sh12 & UMa? & $500 \pm 100$ & Sh12 \\
\hline LHS 2613 & 124249.96 & 415347.0 & $-539.0 \pm 8.0$ & $34.0 \pm 8.0$ & $\mathrm{Z} 13$ & M4.0 & L13 & $10.6 \pm 1.3$ & $\pi$ & vA95 & Argus & $40 \pm 5$ & B14, Sh15 \\
\hline GJ $490 \mathrm{Bab}$ & 125739.35 & 351319.5 & $-281.8 \pm 3.6$ & $-147.0 \pm 6.7$ & $\mathrm{Z} 13$ & M4.5 & $\mathrm{L} 13$ & $19.3 \pm 1.1^{\mathrm{b}}$ & $\pi$ & vL07 & Tuc-Hor? & $35 \pm 5$ & Sh12 \\
\hline GJ $490 \mathrm{Aab}$ & 125740.30 & 351330.6 & $-264.0 \pm 1.9$ & $-150.6 \pm 2.1$ & $\mathrm{Z} 13$ & M1.0 & L13 & $19.3 \pm 1.1$ & $\pi$ & vL07 & Tuc-Hor? & $35 \pm 5$ & Sh12 \\
\hline LHS 2672 & 130247.52 & 413109.9 & $-550.0 \pm 8.0$ & $-165.0 \pm 8.0$ & $\mathrm{Z} 13$ & $\mathrm{M} 3.5^{\mathrm{f}}$ & B98 & $17 \pm 4$ & Phot & L11 & $\ldots$ & $(3-10) \times 10^{3 \mathrm{~d}}$ & B14, Sh15 \\
\hline GJ 1167 A & 130934.95 & 285906.6 & $-338.0 \pm 8.0$ & $-211.0 \pm 8.0$ & $\mathrm{Z} 13$ & M3.5 & Ri06 & $11.5 \pm 2.4$ & $\pi$ & vA95 & Carina? & $30 \pm 5$ & Sh12 \\
\hline LHS 2686 & 131012.69 & 474519.0 & $-643.0 \pm 8.0$ & $-621.0 \pm 8.0$ & Z13 & M4.5 & Ri06 & $13.1 \pm 0.4$ & $\pi$ & D14 & $\ldots$ & $100-500^{\mathrm{i}}$ & B14, Sh15 \\
\hline 2MASS J13233804-2554449 & 132338.05 & -255445.0 & $-556.7 \pm 8.0$ & $-249.4 \pm 8.0$ & $\mathrm{Z} 13$ & M3.5 & Ri06 & $24.0 \pm 0.3$ & $\pi$ & Ri10 & $\ldots$ & $10-300$ & Sh15 \\
\hline 2MASS J13292408-1422122 & 132924.08 & -142212.3 & $103.5 \pm 5.4$ & $-41.0 \pm 5.4$ & Ro10 & M3.5 & Ri06 & $21 \pm 5$ & Phot & Sh12 & $\ldots$ & $20-150$ & Sh09 \\
\hline NLTT $34410 \mathrm{AB}$ & 133239.08 & 305906.5 & $-197.0 \pm 6.6$ & $-82.8 \pm 4.6$ & Z13 & M4.5 & S05b & $20.5 \pm 1.1$ & $\pi$ & D14 & $\ldots$ & $10-300$ & Sh15 \\
\hline 2MASS J14124864-1629561 & 141248.64 & -162956.1 & $-110.2 \pm 1.1$ & $-95.2 \pm 1.5$ & $\mathrm{Z} 13$ & M3.0 & Ri06 & $27 \pm 6$ & Phot & L11 & $\ldots$ & $10-300$ & Sh15 \\
\hline GQ Vir & 141304.92 & -120126.3 & $-601.0 \pm 8.0$ & $-387.3 \pm 8.0$ & $\mathrm{Z} 13$ & M4.5 & Ri06 & $10 \pm 2$ & Phot & L11 & $\ldots$ & $10-300$ & Sh15 \\
\hline 2MASS J14215503-3125537 AB & 142155.04 & -312553.7 & $-165.3 \pm 2.3$ & $-54.4 \pm 2.3$ & $\mathrm{Z} 13$ & M3.9 & Sh09 & $40 \pm 2$ & $\pi$ & Sh12 & $\ldots$ & $35-300$ & Sh09 \\
\hline 2MASS J14442809-0424078 & 144428.10 & -042407.8 & $117.5 \pm 5.0$ & $-99.2 \pm 5.0$ & Ro10 & M2.0 & Ri06 & $29 \pm 10$ & Phot & Sh12 & UMa? & $500 \pm 100$ & Sh12 \\
\hline G $166-49$ & 145110.44 & 310640.7 & $-345.0 \pm 8.0$ & $-181.0 \pm 8.0$ & Z13 & M4.0 & L13 & $12.5 \pm 0.5$ & $\pi$ & D14 & $\ldots$ & 10-300 & Sh15 \\
\hline 1RXS J150907.2+590422 A & 150908.08 & 590425.8 & $98.8 \pm 20.9$ & $-55.9 \pm 20.9$ & Ro08 & M2.5 & Ri06 & $33 \pm 11$ & Phot & Sh12 & & $20-150$ & Sh09 \\
\hline 2MASS J15323737+4653048 & 153237.38 & 465304.9 & $-120.9 \pm 0.8$ & $-8.1 \pm 1.6$ & Z13 & M1.0 & Ri06 & $33 \pm 12$ & Phot & J12 & $\ldots$ & $10-300$ & Sh15 \\
\hline NLTT 40561 & 153350.62 & 251010.6 & $8.0 \pm 8.0$ & $-233.0 \pm 8.0$ & Z13 & M3.5 & Sh09 & $29.1 \pm 1.8$ & $\pi$ & D14 & $\ldots$ & $25-300$ & Sh09 \\
\hline LHS $3122 \mathrm{AB}$ & 154938.33 & 344855.5 & $-663.0 \pm 8.0$ & $688.0 \pm 8.0$ & Z13 & M4.0 & L13 & $17.0 \pm 1.2$ & $\pi$ & vA95 & $\ldots$ & $10-300$ & Sh15 \\
\hline 2MASS J15594729+4403595 AB & 155947.29 & 440359.5 & $-70.7 \pm 0.9$ & $-8.9 \pm 0.6$ & $\mathrm{Z} 13$ & M2.0 & L13 & $27 \pm 2$ & Phot & B14 & $\ldots$ & $50-200$ & B14 \\
\hline 2MASS J16074132-1103073 AB & 160741.33 & -110307.4 & $-69.7 \pm 6.0$ & $-145.6 \pm 6.0$ & Ro10 & M4.0 & Ri06 & $36 \pm 2$ & Kin & M13 & AB Dor? & $120 \pm 10$ & M13 \\
\hline G $202-48$ & 162524.59 & 541814.9 & $442.7 \pm 8.0$ & $-166.6 \pm 9.2$ & $\mathrm{Z13}$ & M2.0 & L13 & $6.52 \pm 0.04$ & $\pi$ & vL07 & $\ldots$ & $1.2-10 \times 10^{3 \mathrm{~d}}$ & B14, Sh15 \\
\hline GJ $3966 \mathrm{AB}$ & 163527.41 & 350057.7 & $143.0 \pm 8.0$ & $-142.0 \pm 8.0$ & Z13 & M4.0 & L13 & $16.2 \pm 0.7$ & $\pi$ & D14 & $\ldots$ & $10-300$ & Sh15 \\
\hline GJ $3997 \mathrm{AB}$ & 171550.10 & 190000.1 & $-132.5 \pm 1.7$ & $13.5 \pm 1.4$ & $\mathrm{Z} 13$ & M0.5 & L13 & $12 \pm 3$ & Phot & L11 & $\ldots$ & $(0.6-10) \times 10^{3 \mathrm{~d}}$ & B14, Sh15 \\
\hline LP $447-38$ AB & 171822.84 & 180856.8 & $-7.7 \pm 5.7$ & $-220.8 \pm 4.0$ & $\mathrm{Z} 13$ & M3.0 & H97 & $26 \pm 4$ & Phot & L11 & $\ldots$ & $(3-10) \times 10^{3}$ & B14, Sh15 \\
\hline GJ 669 B & 171952.98 & 263002.6 & $-217.0 \pm 8.0$ & $354.0 \pm 8.0$ & $\mathrm{Z} 13$ & M4.5 & L13 & $11.8 \pm 0.7$ & $\pi$ & Sh12 & Hyades? & $620 \pm 30$ & Sh12 \\
\hline GJ 669 A & 171954.22 & 263003.0 & $-207.9 \pm 1.1$ & $354.3 \pm 1.2$ & Z13 & M3.5 & L13 & $11.7 \pm 0.7$ & $\pi$ & Sh12 & Hyades? & $620 \pm 30$ & Sh12 \\
\hline LHS 3321 & 174355.95 & 432244.1 & $9.5 \pm 8.0$ & $-602.6 \pm 8.0$ & $\mathrm{Z} 13$ & M2.5 & $\mathrm{L} 13$ & $9.48 \pm 0.11$ & $\pi$ & vL07 & $\ldots$ & $(1.6-10) \times 10^{3}$ & B14, Sh15 \\
\hline G 227-22 & 180216.60 & 641544.6 & $206.0 \pm 8.0$ & $-386.0 \pm 8.0$ & $\mathrm{Z} 13$ & M5.0 & $\mathrm{L} 13$ & $8.5 \pm 0.3$ & $\pi$ & D14 & UMa & $500 \pm 100$ & B14 \\
\hline LP 390-16 & 181306.58 & 260151.9 & $218.0 \pm 8.0$ & $-36.0 \pm 8.0$ & $\mathrm{Z} 13$ & M4.5 & $\mathrm{L} 13$ & $17.2 \pm 0.7$ & $\pi$ & Sh12 & $\ldots$ & 40-300 & Sh09 \\
\hline GJ 9652 A & 191439.26 & 191902.6 & $-617.4 \pm 8.0$ & $434.8 \pm 8.0$ & $\mathrm{Z} 13$ & M3.5 & L13 & $19.1 \pm 1.1$ & $\pi$ & vL07 & $\cdots$ & $60-300$ & Sh09 \\
\hline 2MASS J19303829-1335083 & 193038.30 & -133508.4 & $177.3 \pm 11.3$ & $331.4 \pm 11.3$ & Ro10 & M6.0 & Sh09 & $27.7 \pm 1.2$ & $\pi$ & Sh12 & $\ldots$ & $90-300$ & Sh09 \\
\hline
\end{tabular}


Table 2

(Continued)

\begin{tabular}{|c|c|c|c|c|c|c|c|c|c|c|c|c|c|}
\hline Name & $\alpha_{\mathrm{J} 2000}$ & $\delta_{\mathrm{J} 2000}$ & $\begin{array}{c}\mu_{\alpha} \cos \delta \\
\left(\mathrm{mas} \mathrm{yr}^{-1}\right)\end{array}$ & $\begin{array}{c}\mu_{\delta} \\
\left(\operatorname{mas~yr}^{-1}\right)\end{array}$ & PM Ref & $\begin{array}{l}\text { SpT } \\
( \pm 0.5)\end{array}$ & SpT Ref & $\begin{array}{l}\text { Distance } \\
\quad(\mathrm{pc})\end{array}$ & Method & Dist Ref & $\mathrm{YMG}^{\mathrm{a}}$ & $\begin{array}{l}\text { Age } \\
(\mathrm{Myr})\end{array}$ & Age Ref \\
\hline 1RXS J193528.9+374605 & 193529.23 & 374608.2 & $-134.1 \pm 6.5$ & $-98.7 \pm 4.1$ & Z13 & M3.5 & L13 & $10 \pm 4$ & Phot & Sh12 & & $20-300$ & Sh09 \\
\hline 2MASS J19435432-0546363 & 194354.33 & -054636.4 & $57.4 \pm 7.3$ & $7.3 \pm 8.4$ & Z13 & M4.0 & Ri06 & $32 \pm 2$ & Kin & M13 & Argus? & $40 \pm 5$ & M13 \\
\hline 2MASS J19560294-3207186 AB & 195602.94 & -320718.7 & $35.2 \pm 1.8$ & $-59.9 \pm 1.5$ & $\mathrm{Z} 13$ & M4.0 & Ri06 & $58 \pm 3^{\mathrm{b}}$ & Kin & L09 & $\beta$ Pic? & $23 \pm 3$ & L09, K11 \\
\hline TYC 7443-1102-1 & 195604.38 & -320737.6 & $31.9 \pm 1.4$ & $-65.1 \pm 1.2$ & Z13 & M0.0 & L09 & $58 \pm 3$ & Kin & L09 & $\beta$ Pic? & $23 \pm 3$ & L09, K11 \\
\hline 2MASS J20003177+5921289 AB & 200031.77 & 592129.0 & $74.4 \pm 27.5$ & $21.6 \pm 42.5$ & $\mathrm{Z} 13$ & M4.1 & Sh09 & $20 \pm 5$ & Phot & Sh12 & Castor? & $400 \pm 100$ & Sh12 \\
\hline NLTT 48651 & 200430.78 & -234201.9 & $122.9 \pm 8.0$ & $-339.3 \pm 8.0$ & $\mathrm{Z} 13$ & M4.5 & S05b & $10 \pm 2$ & Phot & L11 & AB Dor? & $120 \pm 10$ & B14, Sh15 \\
\hline 2MASS J20100002-2801410 AB & 201000.03 & -280141.1 & $40.7 \pm 3.0$ & $-62.0 \pm 1.7$ & $\mathrm{Z} 13$ & M3.0 & Ri06 & $45.1 \pm 3.1$ & $\pi$ & Ri14 & $\beta$ Pic & $23 \pm 3$ & M13, Ri14 \\
\hline 2MASS J20284361-1128307 & 202843.62 & -112830.8 & $166.4 \pm 5.2$ & $-93.3 \pm 5.2$ & Ro10 & M3.5 & Ri06 & $18.8 \pm 0.6$ & $\pi$ & $\mathrm{R} 14$ & Argus & $40 \pm 5$ & Sh15 \\
\hline TYC 523-573-1 & 203954.60 & 062011.8 & $89.3 \pm 1.1$ & $-98.4 \pm 1.5$ & $\mathrm{Z} 13$ & K7.5 & L13 & $38.5^{\mathrm{j}}$ & Kin & $\mathrm{Sc} 10$ & AB Dor? & $120 \pm 10$ & $\mathrm{Sc} 10$ \\
\hline NLTT 50066 AB & 205314.65 & -022121.9 & $186.8 \pm 8.0$ & $15.7 \pm 8.0$ & Z13 & M3.0 & Ri06 & $37.9 \pm 5.7$ & $\pi$ & Sh12 & $\ldots$ & $20-300$ & Sh09 \\
\hline NLTT 50710 & 211113.67 & -224817.3 & $-55.4 \pm 8.0$ & $-345.6 \pm 8.0$ & Z13 & M4.0 & Ri06 & $21 \pm 5$ & Phot & L11 & $\ldots$ & $10-300$ & Sh15 \\
\hline GJ 4186 B & 211603.79 & 295146.0 & $219.9 \pm 3.5$ & $45.7 \pm 2.6$ & Z13 & M3.3 & Sh09 & $19.3 \pm 1.1$ & $\pi$ & Sh12 & 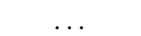 & $25-300$ & Sh09 \\
\hline GJ 4185 Aab & 211605.76 & 295151.1 & $206.0 \pm 2.0$ & $35.3 \pm 2.2$ & Z13 & M4.0 & L13 & $19.5 \pm 1.1$ & $\pi$ & Sh12 & & $25-300$ & Sh09 \\
\hline PYC J21376+0137 AB & 213740.19 & 013713.7 & $80.3 \pm 2.8$ & $-59.4 \pm 3.1$ & Z13 & M4.5 & L13 & $39 \pm 4$ & Kin & Sc12b & $\beta$ Pic? & $23 \pm 3$ & $\mathrm{Sc} 12 \mathrm{c}$ \\
\hline 1RXS J221419.3+253411 AB & 221417.66 & 253406.6 & $167.0 \pm 8.0$ & $-53.0 \pm 8.0$ & Z13 & M4.3 & Sh09 & $28.7 \pm 2.1$ & $\pi$ & Sh12 & Columba? & $30 \pm 5$ & Sh12 \\
\hline GJ 4274 & 222306.97 & -173625.0 & $248.3 \pm 8.0$ & $-895.2 \pm 8.0$ & Z13 & M4.0 & Ri06 & $7.26 \pm 0.09$ & $\pi$ & Ri14 & $\ldots$ & $100-500^{\mathrm{k}}$ & Sh15, Ri14 \\
\hline NLTT 54873 & 224737.65 & 404125.4 & $169.0 \pm 8.0$ & $96.0 \pm 8.0$ & Z13 & M4.0 & Ri06 & $22 \pm 5$ & Phot & Sh12 & $\ldots$ & $35-300$ & Sh09 \\
\hline 2MASS J22581643-1104170 & 225816.44 & -110417.1 & $106.8 \pm 2.9$ & $-5.9 \pm 2.9$ & Z13 & M2.7 & Sh09 & $31.1 \pm 2.9$ & $\pi$ & Sh12 & $\ldots$ & $20-300$ & Sh09 \\
\hline GJ $4338 \mathrm{Bab}$ & 232922.59 & 412752.2 & $421.0 \pm 10.9$ & $-62.2 \pm 12.7$ & Z13 & M4.5 & L13 & $14.7 \pm 0.5$ & $\pi$ & vA95 & 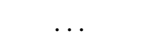 & $40-300$ & Sh09 \\
\hline GJ 4337 A & 232923.46 & 412806.9 & $415.0 \pm 8.0$ & $-41.0 \pm 8.0$ & Z13 & M3.5 & L13 & $14.9 \pm 0.5$ & $\pi$ & vA95 & & $20-300$ & Sh09 \\
\hline GJ 1290 & 234420.84 & 213605.0 & $449.0 \pm 8.0$ & $73.0 \pm 8.0$ & Z13 & M3.4 & Sh09 & $22.0 \pm 2.2$ & $\pi$ & vA95 & $\ldots$ & $25-300$ & Sh09 \\
\hline G 68-46 & 235122.28 & 234420.8 & $265.0 \pm 8.0$ & $-74.0 \pm 8.0$ & Z13 & M4.0 & Ri06 & $21.0 \pm 1.3$ & $\pi$ & D14 & $\ldots$ & $35-300$ & Sh09 \\
\hline 1RXS J235133.3+312720 AB & 235133.67 & 312723.0 & $106.6 \pm 1.7$ & $-87.9 \pm 3.8$ & Z13 & M2.0 & Sh09 & $50 \pm 10$ & Phot & B12b & AB Dor? & $120 \pm 10$ & B12b, Sh12 \\
\hline 1RXS J235452.2+383129 & 235451.47 & 383136.3 & $-130.0 \pm 8.0$ & $-86.0 \pm 8.0$ & Z13 & M4.0 & L13 & $15.9 \pm 0.7$ & $\pi$ & D14 & $\ldots$ & $25-300$ & Sh09 \\
\hline G $158-8$ & 235555.13 & -132123.8 & $308.5 \pm 8.0$ & $21.2 \pm 8.0$ & Z13 & M2.5 & Ri06 & $19 \pm 4$ & Phot & L11 & Argus? & $40 \pm 5$ & M13 \\
\hline GJ $4381 \mathrm{AB}$ & 235749.89 & 383746.9 & $-125.0 \pm 8.0$ & $-138.0 \pm 8.0$ & Z13 & M3.5 & L13 & $21 \pm 8$ & Phot & Sh12 & UMa? & $500 \pm 100$ & Sh12 \\
\hline G $130-31$ & 235919.86 & 324124.5 & $-174.0 \pm 8.0$ & $-241.0 \pm 8.0$ & Z13 & M5.6 & Sh09 & $13 \pm 3$ & Phot & Sh12 & $\ldots$ & 90-300 & Sh09 \\
\hline
\end{tabular}

Notes.

${ }^{a}$ Question marks imply some uncertainty about the young moving group (YMG) status. Usually one kinematic component (radial velocity or distance) is missing so $U V W$ space velocities are ill-constrained along one dimension.

${ }^{\mathrm{b}}$ Distances to the primary stars are adopted for the following companions: the Hipparcos parallax for EX Cet is used for G 271-110; the Hipparcos parallax for GJ 3371 A is used for GJ 3372 B; the parallactic distance to GJ 3577 A (Dittmann et al. 2013) is used for GJ 3578 B; the Hipparcos parallax for GJ 490 A is used for GJ 490 B; the Hipparcos parallax for DX Leo is used for GJ 354.1 B; the kinematic distance to TYC 7443-1102-1 (Lépine \& Simon 2009) is used for 2MASS J19560294-3207186 AB.

${ }^{\mathrm{c}}$ Spectral types are estimated from photometry (see associated reference) and have uncertainties of \pm 2 subclasses.

d Age based on the activity-lifetime relation from West et al. (2008).

e The age for GJ 3577 A from Shkolnik et al. (2009) is adopted for the companion GJ 3578 B.

${ }^{\mathrm{f}}$ Spectral type uncertainties are \pm 1 subclass.

$\mathrm{g}$ This distance is the photometric distance to the M8.5 companion 2MASS J11240487+3808054 (Appendix C).

${ }^{\mathrm{h}}$ Spectral type and distance are estimated using the $V-J$ relations from Lépine et al. (2013). The uncertainty in spectral type is one subclass.

${ }^{\mathrm{i}}$ The minimum ages of these stars are ZAMS since they lie on the main sequence in the $\left\{M_{V}, V-K_{S}\right\}$ diagram.

j No distance uncertainty is provided in Schlieder et al. (2010).

${ }^{\mathrm{k}}$ The position of GJ 4274 (LHS 3799) on the HR diagram (Riedel et al. 2014) implies a minimum age ZAMS age of $\sim 100$ Myr.

References. (A13) Allers \& Liu 2013; (B12a) Bowler et al. 2012b; (B14) this work; (B98) Buscombe 1998; (D14) Dittmann et al. 2013; (H97) Hawley et al. 1996, Reid et al. 1995; (J12) Janson et al. 2012; (K11) Kiss et al. 2010; (K08) Kirkpatrick et al. 2008; (M13) Malo et al. 2013; (L05) Lowrance et al. 2005; (Lo10) Looper et al. 2010b; (L09) Lépine \& Simon 2009; (L11) Lépine \& Gaidos 2011; (L13) Lépine et al. 2013; (L08) Law et al. 2008; (Re06) Reid \& Walkowicz 2006; (Re07) Reid et al. 2007 (Ri06) Riaz et al. 2006; (Ri10) Riedel et al. 2010; (Ri11) Riedel et al. 2011; (Ri14) Riedel et al. 2014; (R11) Röser et al.

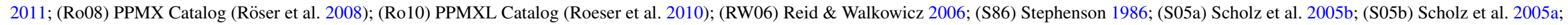

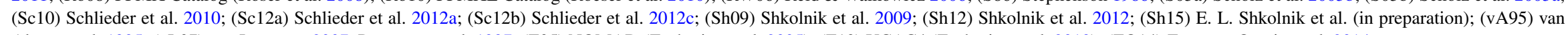
Altena et al. 1995; (vL07) van Leeuwen 2007, Perryman et al. 1997; (Z05) NOMAD (Zacharias et al. 2005); (Z13) UCAC4 (Zacharias et al. 2013 ); (ZO14) Zapatero Osorio et al. 2014. 
the sample) are within $30 \mathrm{pc}$ and 114 (93\% of the sample) are within 40 pc.

When possible we avoided stars with low galactic latitudes where background contamination rates are high. Targets near the galactic plane were generally only observed if an RA gap existed in the target list for any particular night. This preference is reflected in the relative dearth of targets for $|b| \lesssim 20^{\circ}$ in Figure 1. The distribution of $R$-band magnitudes ranges from $\approx 10-15 \mathrm{mag}$ and is roughly divided into two bins according to observability with NGS-AO at Subaru $(\lesssim 13 \mathrm{mag})$ and Keck ( $\lesssim 15 \mathrm{mag}$ ). The cumulative age distribution of our sample is shown in Figure 1: $50 \%$ of stars are younger than $135 \mathrm{Myr}$ and $90 \%$ are younger than $620 \mathrm{Myr}$.

\section{OBSERVATIONS}

\subsection{Keck II/NIRC2 Adaptive Optics Imaging}

We carried out our observations at the Keck II 10 m telescope with the facility near-infrared imaging camera NIRC2 using natural guide star adaptive optics (NGS-AO; Wizinowich et al. 2000) between 2010 August and 2013 August (Table 3). A single target, NLTT 13844 ( $R \sim 14.8 \mathrm{mag}$ ), was observed with laser guide star AO (LGS-AO; Wizinowich et al. 2006; van Dam et al. 2006). Most of our imaging was carried out with the narrow camera, which has a plate scale of $9.952 \pm 0.002$ mas pixel ${ }^{-1}$ (Yelda et al. 2010) and provides Nyquist sampling at the diffraction limit beyond $\sim 1.2 \mu \mathrm{m}$. In this mode, the field of view (FOV) across the array's $1024 \times 1024$ pixels is $10^{\prime \prime} .2 \times$ $10^{\prime}$. 2 . When conditions were good (seeing below $\sim 1^{\prime \prime}$ ) we used the Mauna Kea Observatory (MKO) $H$-band filter (Simons \& Tokunaga 2002; Tokunaga \& Vacca 2005) as a compromise between higher Strehl and increased sky background levels at longer wavelengths. When conditions were below average, we used the $K_{S}$ filter to benefit from better AO correction.

We first obtained short, unsaturated images of each target to check for stellar multiplicity. Binary systems were generally skipped, although in a few cases close companions were only resolved in our second-epoch imaging. For single stars we typically obtained 40 minutes of total on-source integration time (usually 40 frames each with $60 \mathrm{~s}$ exposures and 1 coadd reading out with multiple correlated double sampling) in Angular Differential Imaging mode (ADI; Liu 2004; Marois et al. 2006) after centering the target behind the partly opaque ( $\Delta$ $H \sim 6 \mathrm{mag}$ ) $600 \mathrm{mas}$ diameter coronagraph. To avoid the lowerleft quadrant of NIRC2, which suffers from elevated noise levels, we positioned the coronagraph at column 616 (the occulting spot is fixed in $y$ at row 430). Raw images were first cleaned of bad pixels and cosmic rays then flat-fielded to remove pixel-to-pixel sensitivity variations.

This results in an inner working angle (IWA) of 300 mas and an outer working angle (OWA) between $\approx 4^{\prime \prime}$ (for complete spatial coverage) and 8..5 (for partial coverage). The NIRC2 coronagraph is particularly useful for image registration and photometric calibration since the star is visible behind the mask. Corrections for differential atmospheric refraction were applied during most of the observations to keep the star centered behind the coronagraph. To further monitor the quality of AO correction, we also obtained a set of unsaturated frames immediately before and after our ADI sequences.

The scheduling of our ADI observations were optimized to maximize rotation at small separations $\left(\sim 0^{\prime} .5\right)$ but minimize blurring at modest separations $\left(\sim 3^{\prime \prime}\right)$. This compromise ensures that physical companions will have undergone enough rotation on the detector to avoid strong self-subtraction during postprocessing. This strategy also reduces sensitivity losses at several arcseconds caused by smearing when rotation rates are high, which can occur near transit for declinations near the observing site's latitude $\left(+20^{\circ}\right.$ for Mauna Kea; see Biller et al. 2008 for a detailed discussion of these effects). In practice it is difficult to strictly adhere to these constraints, but most of the FOV rotations are near the desired values of $\sim 15^{\circ}-40^{\circ}$.

Follow-up second-epoch observations were carried out in ADI mode, standard imaging mode with the telescope rotator on, and with the wide $\left(0^{\prime \prime} .04\right.$ pixel $^{-1} ; 40^{\prime \prime}$ FOV $)$ and narrow NIRC2 camera modes depending on the candidate being recovered. Integration times were generally much shorter than first-epoch exposures since it is often not necessary to achieve the same limiting depth to recover faint candidates.

For the NIRC2 narrow camera, we adopt the plate scale measurement of $9.952 \pm 0.002$ mas pixel ${ }^{-1}$ and orientation of $+0.252 \pm 0.009$ found by Yelda et al. (2010). Post-fit residuals for the NIRC2 narrow camera distortion solution made available by Keck Observatory are $\approx 0.6$ mas (B. Cameron, 2007, private communication).

\subsection{Subaru/HiCIAO Adaptive Optics Imaging}

Our NGS-AO observations at the $8.2 \mathrm{~m}$ Subaru Telescope were obtained with the AO188 adaptive optics system (Hayano et al. 2010) coupled with the High Contrast Instrument for the Subaru Next Generation Adaptive Optics (HiCIAO) imaging instrument (Hodapp et al. 2008; Suzuki et al. 2010; Table 3). Our observing strategy with HiCIAO was similar to that with NIRC2. ADI observations were carried out with the star centered behind the 300 mas diameter opaque Lyot coronagraph. The $H$ (MKO) and $K_{S}$ filters were used for our deep imaging with typical on-source integration times of 40 minutes $(1$ coadd $\times$ $60 \mathrm{~s} \times 40$ frames). Sets of short unsaturated frames were taken before, in the middle, and after each ADI sequence to monitor AO correction and photometrically calibrate our data. The atmospheric dispersion corrector for the AO188 (Egner et al. 2010) was employed to minimize drifting caused by changing airmass. With a plate scale of $9.723 \pm 0.011$ mas pixel $^{-1}$ in the $H$ band (Appendix A), the $2048 \times 2048$ pixel HAWAII-2RG detector corresponds to a FOV of 20'5. For our deep coronagraphic data the IWA is 0 .'2 and the OWA is $\approx 10^{\prime \prime}$ $\left(\approx 14^{\prime \prime}\right)$ for full (partial) coverage. Dome flats and bias frames were obtained at the start and end of each observing run.

The raw HiCIAO images suffer from horizontal and vertical electronic readout structure imprinted in each image, which corresponds to 32 readout channels with different voltages (Suzuki et al. 2010; Brandt et al. 2013). To remove these random and changing bias stripes we use a procedure developed by the Subaru Strategic Exploration of Exoplanets and Disks (SEEDS; Tamura et al. 2006) team (R. Kandori 2011, private communication), which is based on measuring and subtracting these patterns in the science data itself. To further remove residual patterns, we subtract the median-combined horizontal and vertical profiles after masking out the science target in each image. Together these eliminate nearly all systematic features caused by the electronics. After bias subtraction, cosmic rays and bad pixels are removed and the images are divided by a normalized flat field.

Seeing was poor $\left(1^{\prime \prime}-2^{\prime \prime}\right)$ during most of our HiCIAO observations. This significantly degraded the AO correction and the limiting contrasts for many of our targets. While we attempted to 
Table 3

Summary of Coronagraphic Observations

\begin{tabular}{|c|c|c|c|c|c|c|c|c|c|c|}
\hline Name & 2MASS ID & $\begin{array}{l}\text { UT Date } \\
\text { (Y-M-D) }\end{array}$ & Instrument $^{\mathrm{a}}$ & Filter & $\begin{array}{c}\text { Cor. } \\
{\text { (mas })^{b}}^{\text {b }}\end{array}$ & $\begin{array}{l}\text { No. of } \\
\text { Exp. }\end{array}$ & $\begin{array}{l}\text { Exp. Time } \\
\text { (minutes) }^{c}\end{array}$ & Mode $^{\mathrm{d}}$ & $\begin{array}{c}\text { Rotation } \\
\left({ }^{\circ}\right)\end{array}$ & $\begin{array}{c}\text { Strehl } \\
(\%)\end{array}$ \\
\hline \multirow[t]{2}{*}{ GJ $3030 \mathrm{AB}$} & J00215781+4912379 & 2011 Dec 27 & HiCIAO & $H$ & 300 & 39 & 39.0 & ADI & 26.4 & $\ldots$ \\
\hline & & 2013 Aug 17 & NIRC2/W & $K_{S}$ & 600 & 4 & 0.33 & ADI & 0.5 & $\ldots$ \\
\hline \multirow[t]{2}{*}{ NLTT 1875} & J00350487+5953079 & 2010 Aug 18 & $\mathrm{NIRC} 2 / \mathrm{N}$ & $H$ & 600 & 40 & 40.0 & ADI & 16.5 & 26 \\
\hline & & 2011 Aug 20 & $\mathrm{NIRC} 2 / \mathrm{N}$ & $H$ & 600 & 10 & 10.0 & DI & $\ldots$ & $\ldots$ \\
\hline G 132-50 Aab & $\mathrm{J} 01034013+4051288$ & 2011 Dec 28 & HiCIAO & $K_{S}$ & 300 & 40 & 40.0 & ADI & 34.1 & $\ldots$ \\
\hline G 172-56 & J01291257+4819354 & 2010 Aug 17 & $\mathrm{NIRC} 2 / \mathrm{N}$ & $H$ & 600 & 41 & 41.0 & ADI & 22.6 & 22 \\
\hline G 272-43 & J01335800-1738235 & 2012 Jan 3 & HiCIAO & $K_{S}$ & 300 & 40 & 40.0 & ADI & 19.5 & $\ldots$ \\
\hline \multirow[t]{3}{*}{ G 271-110 } & J01365529-0647363 & 2010 Aug 18 & $\mathrm{NIRC} 2 / \mathrm{N}$ & $H$ & 600 & 36 & 36.0 & ADI & 17.5 & 32 \\
\hline & & 2012 Aug 23 & $\mathrm{NIRC} 2 / \mathrm{N}$ & $K_{S}$ & 600 & 20 & 20.0 & DI & $\ldots$ & 30 \\
\hline & & 2013 Feb 4 & $\mathrm{NIRC} 2 / \mathrm{N}$ & $H$ & 600 & 40 & 40.0 & DI & $\ldots$ & 4 \\
\hline TYC 1752-63-1 & $\mathrm{J} 01372322+2657119$ & 2012 Aug 23 & $\mathrm{NIRC} 2 / \mathrm{N}$ & $K_{S}$ & 600 & 40 & 40.0 & ADI & 16.5 & 30 \\
\hline G 272-115 & J01531133-2105433 & 2011 Dec 27 & HiCIAO & $H$ & 300 & 40 & 40.0 & ADI & 14.1 & $\ldots$ \\
\hline \multirow[t]{2}{*}{ NLTT 6549 AB } & J01581361+4844197 & 2011 Jan 26 & HiCIAO & $H$ & 300 & 42 & 42.0 & ADI & 18.1 & $\ldots$ \\
\hline & & 2011 Aug 20 & $\mathrm{NIRC} 2 / \mathrm{N}$ & $H$ & 600 & 40 & 40.0 & ADI & 22.6 & 35 \\
\hline GJ 3136 & J02085359+4926565 & 2011 Jan 27 & $\mathrm{HiCIAO}$ & $K_{S}$ & 300 & 40 & 40.0 & ADI & 17.6 & $\cdots$ \\
\hline LP 353-51 & $\mathrm{J} 02232663+2244069$ & 2011 Dec 28 & HiCIAO & $K_{S}$ & 300 & 40 & 40.0 & ADI & 12.2 & $\ldots$ \\
\hline \multirow[t]{2}{*}{ 1RXS J022735.8+471021 } & $\mathrm{J} 02273726+4710045$ & 2010 Aug 17 & $\mathrm{NIRC} 2 / \mathrm{N}$ & $H$ & 600 & 40 & 40.0 & ADI & 14.2 & 27 \\
\hline & & 2013 Feb 4 & $\mathrm{NIRC} 2 / \mathrm{N}$ & $H$ & 600 & 35 & 35.0 & DI & $\ldots$ & 11 \\
\hline 1RXS J023138.7+445640 & J02313926+4456387 & 2010 Aug 18 & $\mathrm{NIRC} 2 / \mathrm{N}$ & $H$ & 600 & 39 & 39.0 & ADI & 22.8 & 38 \\
\hline G 75-35 & J02411510-0432177 & 2012 Jan 3 & HiCIAO & $H$ & 300 & 40 & 40.0 & ADI & 17.7 & $\ldots$ \\
\hline 2MASS J03033668-2535329 AB & J03033668-2535329 & 2011 Dec 28 & HiCIAO & $K_{S}$ & 300 & 20 & 20.0 & ADI & 7.1 & $\ldots$ \\
\hline \multirow[t]{5}{*}{ LP $247-13$} & J03153783+3724143 & 2011 Jan 26 & HiCIAO & $K_{S}$ & 300 & 40 & 40.0 & ADI & 14.2 & $\ldots$ \\
\hline & & 2011 Aug 20 & $\mathrm{NIRC} 2 / \mathrm{N}$ & $H$ & 600 & 10 & 10.0 & DI & $\ldots$ & 38 \\
\hline & & 2011 Aug 20 & $\mathrm{NIRC} 2 / \mathrm{N}$ & $K_{S}$ & 600 & 6 & 3.0 & DI & $\ldots$ & 58 \\
\hline & & 2012 Jan 14 & $\mathrm{NIRC} 2 / \mathrm{N}$ & $H$ & 600 & 5 & 5.0 & DI & $\ldots$ & $\ldots$ \\
\hline & & 2012 Jan 14 & $\mathrm{NIRC} 2 / \mathrm{N}$ & $K$ & 600 & 5 & 5.0 & DI & $\ldots$ & $\ldots$ \\
\hline GJ 3287 & J04274130+5935167 & 2013 Feb 4 & $\mathrm{NIRC} 2 / \mathrm{N}$ & $K_{S}$ & 600 & 40 & 40.0 & ADI & 15.9 & 41 \\
\hline LP 834-32 & J04353618-2527347 & 2011 Dec 28 & HiCIAO & $K_{S}$ & 300 & 40 & 40.0 & ADI & 16.0 & $\ldots$ \\
\hline \multirow[t]{2}{*}{ NLTT 13844} & $\mathrm{~J} 04450562+4324342$ & 2012 Oct 8 & $\mathrm{NIRC} 2 / \mathrm{N}^{\mathrm{f}}$ & $H$ & 600 & 10 & 10.0 & DI & $\ldots$ & 17 \\
\hline & & 2013 Jan 17 & $\mathrm{NIRC} 2 / \mathrm{N}^{\mathrm{f}}$ & $K$ & 600 & 5 & 5.0 & DI & $\ldots$ & $\ldots$ \\
\hline \multirow[t]{3}{*}{ 1RXS J045101.0+312734 } & J04510138+3127238 & 2011 Jan 27 & HiCIAO & $K_{S}$ & 300 & 40 & 40.0 & ADI & 13.9 & $\ldots$ \\
\hline & & 2011 Dec 28 & HiCIAO & $K_{S}$ & 300 & 40 & 40.0 & ADI & 26.4 & $\ldots$ \\
\hline & & 2012 Jan 3 & HiCIAO & $K_{S}$ & 300 & 20 & 20.0 & ADI & 3.60 & $\cdots$ \\
\hline L 449-1 AB & J05172292-3521545 & 2011 Dec 28 & HiCIAO & $K_{S}$ & 300 & 27 & 27.0 & ADI & 8.5 & $\cdots$ \\
\hline \multirow[t]{2}{*}{ 1RXS J055446.0+105559 } & J05544574+1055570 & 2011 Jan 27 & HiCIAO & $K_{S}$ & 300 & 40 & 40.0 & ADI & 5.0 & $\ldots$ \\
\hline & & 2013 Feb 4 & $\mathrm{NIRC} 2 / \mathrm{N}$ & $K_{S}$ & 600 & 40 & 40.0 & ADI & 17.5 & 46 \\
\hline GJ $3371 \mathrm{~A}$ & J05593774+5835351 & 2011 Dec 27 & HiCIAO & $H$ & 300 & 74 & 37.0 & ADI & 18.9 & $\ldots$ \\
\hline GJ $3372 \mathrm{~B}$ & J05595569+5834155 & 2011 Mar 25 & NIRC2/N & $K_{S}$ & 600 & 50 & 50.0 & ADI & 20.6 & 44 \\
\hline \multirow[t]{3}{*}{ AP Col } & $\mathrm{J} 06045215-3433360$ & 2011 Dec 27 & HiCIAO & $K_{S}$ & 300 & 60 & 60.0 & ADI & 21.1 & $\cdots$ \\
\hline & & 2012 Jan 3 & HiCIAO & $H$ & 300 & 40 & 40.0 & ADI & 13.6 & $\cdots$ \\
\hline & & 2012 Oct 8 & NIRC2/W & $H$ & 1500 & 9 & 4.5 & DI & $\ldots$ & $\ldots$ \\
\hline G 249-36 & J06052936+6049231 & 2011 Jan 27 & HiCIAO & $K_{S}$ & 300 & 40 & 40.0 & ADI & 14.2 & $\ldots$ \\
\hline 2MASS J06180730+7506032 & J06180730+7506032 & 2012 Jan 3 & HiCIAO & $H$ & 300 & 40 & 40.0 & ADI & 13.3 & $\ldots$ \\
\hline GJ 3395 & J06310116+5002485 & 2011 Jan 26 & HiCIAO & $K_{S}$ & 300 & 40 & 40.0 & ADI & 23.6 & $\ldots$ \\
\hline \multirow[t]{2}{*}{ G $108-36$} & J06515901+0312553 & 2011 Jan 27 & HiCIAO & $K_{S}$ & 300 & 40 & 40.0 & ADI & 13.9 & $\cdots$ \\
\hline & & 2012 Jan 3 & HiCIAO & $K_{S}$ & 300 & 29 & 29.0 & ADI & 28.4 & $\ldots$ \\
\hline \multirow[t]{4}{*}{ 1RXS J073829.3+240014 } & J07382951+2400088 & 2010 Nov 27 & $\mathrm{NIRC} 2 / \mathrm{N}$ & $H$ & 600 & 42 & 42.0 & ADI & 7.6 & 30 \\
\hline & & 2011 Dec 27 & HiCIAO & $K_{S}$ & 300 & 40 & 40.0 & ADI & 20.4 & $\ldots$ \\
\hline & & 2012 Jan 3 & HiCIAO & $K_{S}$ & 300 & 40 & 40.0 & ADI & 5.7 & $\ldots$ \\
\hline & & 2013 Jan 17 & $\mathrm{NIRC} 2 / \mathrm{N}$ & $K$ & 600 & 8 & 8.0 & DI & $\ldots$ & $\ldots$ \\
\hline 1RXS J091744.5+461229 AB & J09174473+4612246 & 2011 Dec 28 & HiCIAO & $K_{S}$ & 300 & 40 & 40.0 & ADI & 18.4 & $\ldots$ \\
\hline GJ $354.1 \mathrm{~B}$ & J09324827+2659443 & 2013 May 2 & $\mathrm{NIRC} 2 / \mathrm{N}$ & $K_{S}$ & 600 & 40 & 40.0 & ADI & 19.5 & 32 \\
\hline PYC J09362+3731 AB & J09361593+3731456 & 2012 May 22 & $\mathrm{NIRC} 2 / \mathrm{N}$ & $H$ & 600 & 105 & 35.0 & ADI & 16.9 & 9 \\
\hline G 161-71 & J09445422-1220544 & 2011 Nov 16 & $\mathrm{NIRC} 2 / \mathrm{N}$ & $H$ & 600 & 37 & 37.0 & ADI & 61.7 & 30 \\
\hline NLTT $22741 \mathrm{~A}$ & J09510459+3558098 & 2012 May 21 & $\mathrm{NIRC} 2 / \mathrm{N}$ & $K_{S}$ & 600 & 40 & 40.0 & ADI & 11.8 & 8 \\
\hline GJ 3577 A & J09591880+4350256 & 2013 Feb 4 & $\mathrm{NIRC} 2 / \mathrm{N}$ & $K_{S}$ & 600 & 40 & 40.0 & ADI & 26.3 & 30 \\
\hline GJ 3578 B & J09592093+4350258 & 2013 Feb 4 & $\mathrm{NIRC} 2 / \mathrm{N}$ & $K_{S}$ & 600 & 40 & 40.0 & ADI & 22.6 & 39 \\
\hline G 196-3 A & $\mathrm{J} 10042148+5023135$ & 2011 Jan 26 & HiCIAO & $K_{S}$ & 300 & 40 & 40.0 & ADI & 10.7 & $\cdots$ \\
\hline GJ 2079 AB & J10141918+2104297 & 2011 Dec 28 & HiCIAO & $K_{S}$ & 300 & 40 & 40.0 & ADI & 22.9 & $\ldots$ \\
\hline & & 2013 Feb 4 & NIRC2 & $K_{S}$ & 600 & 62 & 31.0 & ADI & 14.5 & 26 \\
\hline GJ 3629 AB & $\mathrm{J} 10512059+3607255$ & 2012 May 10 & HiCIAO & $H$ & 300 & 20 & 20.0 & ADI & 17.5 & $\ldots$ \\
\hline GJ 3639 & $\mathrm{~J} 11031000+3639085$ & 2011 Jan 27 & $\mathrm{HiCIAO}$ & $K_{S}$ & 300 & 40 & 40.0 & ADI & 38.3 & $\cdots$ \\
\hline NLTT 26114 & $\mathrm{~J} 11032125+1337571$ & 2012 Jan 14 & NIRC2/N & $K$ & 600 & 32 & 32.0 & ADI & 15.3 & $\ldots$ \\
\hline
\end{tabular}


Table 3

(Continued)

\begin{tabular}{|c|c|c|c|c|c|c|c|c|c|c|}
\hline Name & 2MASS ID & $\begin{array}{l}\text { UT Date } \\
\text { (Y-M-D) }\end{array}$ & Instrument $^{\mathrm{a}}$ & Filter & $\begin{array}{l}\text { Cor. } \\
(\mathrm{mas})^{\mathrm{b}}\end{array}$ & $\begin{array}{l}\text { No. of } \\
\text { Exp. }\end{array}$ & $\begin{array}{l}\text { Exp. Time } \\
\text { (minutes) }^{\mathrm{c}}\end{array}$ & Mode $^{\mathrm{d}}$ & $\begin{array}{l}\text { Rotation } \\
\left({ }^{\circ}\right)\end{array}$ & $\begin{array}{c}\text { Strehl }^{\mathrm{e}} \\
(\%)\end{array}$ \\
\hline \multirow[t]{2}{*}{ NLTT 26359} & J11072772-1917293 & 2011 Dec 27 & HiCIAO & $K_{S}$ & 300 & 40 & 40.0 & ADI & 14.9 & $\ldots$ \\
\hline & & 2013 May 2 & NIRC2/W & $K_{S}$ & 1500 & 5 & 2.5 & DI & & $\ldots$ \\
\hline G $119-62$ & $\mathrm{~J} 11115215+3332110$ & $2011 \operatorname{Jan} 26$ & HiCIAO & $K_{S}$ & 300 & 40 & 40.0 & ADI & 18.3 & $\ldots$ \\
\hline \multirow{2}{*}{ 2MASS J11240434+3808108 } & $\mathrm{J} 11240434+3808108$ & 2010 Nov 28 & $\mathrm{NIRC} 2 / \mathrm{N}$ & $H$ & 600 & 51 & 34.0 & ADI & 19.0 & 17 \\
\hline & & 2011 Mar 25 & NIRC2/N & $K_{S}$ & 600 & 42 & 42.0 & ADI & 16.0 & 54 \\
\hline TWA $30 \mathrm{~A}$ & J11321831-3019518 & 2011 Mar 25 & NIRC2/N & $K_{S}$ & 600 & 20 & 20.0 & ADI & 7.9 & 20 \\
\hline G $10-52$ & J11483548+0741403 & 2011 Jan 27 & HiCIAO & $K_{S}$ & 300 & 40 & 40.0 & ADI & 15.3 & $\ldots$ \\
\hline LP 734-34 & J12102834-1310234 & 2011 Dec 27 & HiCIAO & $K_{S}$ & 300 & 40 & 40.0 & ADI & 17.0 & $\ldots$ \\
\hline \multirow[t]{3}{*}{ G $13-33 \mathrm{AB}$} & J12225061-0404462 & 2011 Dec 28 & HiCIAO & $K_{S}$ & 300 & 39 & 39.0 & ADI & 26.1 & $\ldots$ \\
\hline & & 2012 May 21 & NIRC2/N & $K_{S}$ & 600 & 30 & 30.0 & ADI & 11.9 & 12 \\
\hline & & 2013 Feb 4 & $\mathrm{NIRC} 2 / \mathrm{N}$ & $K_{S}$ & 600 & 34 & 34.0 & DI & $\ldots$ & 25 \\
\hline \multirow[t]{3}{*}{ GJ 3729 AB } & J12290290+4143497 & 2011 Jan 26 & HiCIAO & $K_{S}$ & 300 & 45 & 45.0 & ADI & 24.5 & $\ldots$ \\
\hline & & 2011 Jun 21 & NIRC2/N & $H$ & 600 & 35 & 35.0 & ADI & 20.9 & 19 \\
\hline & & 2012 Jan 3 & HiCIAO & $H$ & 300 & 47 & 47.0 & ADI & 33.7 & $\ldots$ \\
\hline \multirow[t]{3}{*}{ 1RXS J124147.5+564506 } & $\mathrm{J} 12414736+5645137$ & 2011 Jan 26 & HiCIAO & $K$ & 300 & 40 & 40.0 & ADI & 20.6 & $\ldots$ \\
\hline & & 2012 May 10 & HiCIAO & $K_{S}$ & 300 & 40 & 40.0 & ADI & 18.5 & $\ldots$ \\
\hline & & 2013 May 2 & $\mathrm{NIRC} 2 / \mathrm{W}$ & $K_{S}$ & 1500 & 5 & 4.2 & DI & $\ldots$ & $\ldots$ \\
\hline \multirow[t]{2}{*}{ LHS 2613} & J12424996+4153469 & 2011 Dec 27 & HiCIAO & $K_{S}$ & 300 & 20 & 20.0 & ADI & 12.6 & $\ldots$ \\
\hline & & 2012 Jan 3 & HiCIAO & $H$ & 300 & 40 & 40.0 & ADI & 20.4 & $\ldots$ \\
\hline LHS 2672 & $\mathrm{~J} 13024751+4131098$ & 2012 May 10 & HiCIAO & $H$ & 300 & 40 & 40.0 & ADI & 20.8 & $\ldots$ \\
\hline \multirow[t]{2}{*}{ GJ $1167 \mathrm{~A}$} & $\mathrm{~J} 13093495+2859065$ & 2011 Jan 27 & HiCIAO & $K_{S}$ & 300 & 40 & 40.0 & ADI & 21.0 & $\ldots$ \\
\hline & & 2012 May 10 & HiCIAO & $K_{S}$ & 300 & 40 & 40.0 & ADI & 20.4 & $\ldots$ \\
\hline LHS 2686 & $\mathrm{~J} 13101268+4745190$ & 2011 Jun 21 & $\mathrm{NIRC} 2 / \mathrm{N}$ & $H$ & 600 & 40 & 40.0 & ADI & 18.4 & 34 \\
\hline 2MASS J13233804-2554449 & J13233804-2554449 & 2013 Feb 4 & NIRC2/N & $K_{S}$ & 600 & 40 & 40.0 & ADI & 15.0 & 31 \\
\hline 2MASS J13292408-1422122 & J13292408-1422122 & 2011 Mar 25 & NIRC2/N & $K_{S}$ & 600 & 52 & 52.0 & ADI & 15.0 & 53 \\
\hline \multirow[t]{2}{*}{ 2MASS J14124864-1629561 } & J14124864-1629561 & 2012 May 22 & NIRC2/N & $K_{S}$ & 600 & 40 & 40.0 & ADI & 15.6 & 28 \\
\hline & & 2013 May 2 & NIRC2/N & $K_{S}$ & 600 & 40 & 40.0 & ADI & 18.1 & 49 \\
\hline GQ Vir & J14130492-1201262 & 2012 May 21 & $\mathrm{NIRC} 2 / \mathrm{N}$ & $K_{S}$ & 600 & 40 & 40.0 & ADI & 19.2 & 18 \\
\hline 2MASS J14442809-0424078 & $\mathrm{J} 14442809-0424078$ & 2011 Mar 25 & $\mathrm{NIRC} 2 / \mathrm{N}$ & $K_{S}$ & 600 & 40 & 40.0 & ADI & 10.3 & 51 \\
\hline \multirow[t]{2}{*}{ G $166-49$} & $\mathrm{~J} 14511044+3106406$ & 2012 May 22 & NIRC2/N & $H$ & 600 & 80 & 40.0 & ADI & 44.9 & 24 \\
\hline & & 2013 Feb 4 & NIRC2/N & $K_{S}$ & 600 & 20 & 20.0 & DI & & 53 \\
\hline 1RXS J150907.2+590422 A & $\mathrm{J} 15090696+5904282$ & 2012 May 21 & NIRC2/N & $K_{S}$ & 600 & 40 & 40.0 & ADI & 14.9 & 22 \\
\hline \multirow[t]{2}{*}{ 2MASS J15323737+4653048 } & $\mathrm{J} 15323737+4653048$ & 2012 May 22 & NIRC2/N & $K_{S}$ & 600 & 33 & 33.0 & ADI & 13.4 & 13 \\
\hline & & 2013 Aug 17 & NIRC2/N & $K_{S}$ & 600 & 40 & 40.0 & ADI & 14.9 & 49 \\
\hline NLTT 40561 & $\mathrm{~J} 15335062+2510106$ & 2010 Aug 18 & NIRC2/N & $H$ & 600 & 40 & 40.0 & ADI & 5.8 & 31 \\
\hline G 202-48 & $\mathrm{J} 16252459+5418148$ & 2011 Jun 21 & NIRC2/N & $H$ & 600 & 20 & 20.0 & ADI & 11.9 & 37 \\
\hline \multirow[t]{2}{*}{ GJ $3966 \mathrm{AB}$} & $\mathrm{J} 16352740+3500577$ & 2011 Jun 21 & $\mathrm{NIRC} 2 / \mathrm{N}$ & $H$ & 600 & 10 & 10.0 & ADI & 10.2 & 18 \\
\hline & & 2012 Apr 12 & NIRC2/N & $H$ & 600 & 3 & 3.0 & DI & $\ldots$ & $\ldots$ \\
\hline LP 447-38 AB & J17182284+1808568 & 2012 May 10 & HiCIAO & $H$ & 300 & 10 & 10.0 & ADI & 4.1 & $\ldots$ \\
\hline GJ 669 B & $\mathrm{J} 17195298+2630026$ & 2010 Aug 18 & NIRC2/N & $H$ & 600 & 41 & 43.7 & ADI & 15.0 & 31 \\
\hline \multirow[t]{2}{*}{ GJ 669 A } & $\mathrm{J} 17195422+2630030$ & 2012 May 10 & HiCIAO & $H$ & 300 & 40 & 40.0 & ADI & 16.2 & $\ldots$ \\
\hline & & 2013 May 2 & NIRC2/W & $K_{S}$ & 1500 & 5 & 4.2 & DI & $\ldots$ & $\ldots$ \\
\hline LHS 3321 & $\mathrm{~J} 17435595+4322441$ & 2011 Jun 21 & NIRC2/N & $H$ & 600 & 80 & 40.0 & ADI & 36.5 & 37 \\
\hline & & 2012 May 10 & HiCIAO & $H$ & 300 & 40 & 40.0 & ADI & 19.9 & $\ldots$ \\
\hline G 227-22 & $\mathrm{J} 18021660+6415445$ & 2010 Aug 17 & NIRC2/N & $H$ & 600 & 30 & 30.0 & ADI & 17.9 & 26 \\
\hline LP 390-16 & $\mathrm{J} 18130657+2601519$ & 2011 Mar 25 & NIRC2/N & $K_{S}$ & 600 & 71 & 71.0 & ADI & 33.7 & 53 \\
\hline GJ 9652 A & J19143925+1919025 & 2011 Aug 20 & $\mathrm{NIRC} 2 / \mathrm{N}$ & $H$ & 600 & 84 & 28.0 & ADI & 1.5 & 25 \\
\hline & & 2012 Aug 23 & NIRC2/N & $H$ & 600 & 59 & 19.7 & ADI & 27.7 & 17 \\
\hline 2MASS J19303829-1335083 & J19303829-1335083 & 2010 Aug 17 & NIRC $2 / \mathrm{N}$ & $H$ & 600 & 46 & 46.0 & ADI & 22.0 & 23 \\
\hline & & $2011 \mathrm{Jul} 1$ & NIRC2/N & $H$ & 600 & 40 & 40.0 & ADI & 15.4 & 28 \\
\hline 1RXS J193528.9+374605 & $\mathrm{J} 19352922+3746082$ & 2010 Aug 17 & NIRC2/N & $H$ & 600 & 36 & 36.0 & ADI & 18.9 & 32 \\
\hline & & 2011 Aug 20 & NIRC $2 / \mathrm{N}$ & $H$ & 600 & 20 & 20.0 & ADI & 25.5 & 42 \\
\hline 2MASS J19435432-0546363 & J19435432-0546363 & 2012 May 21 & NIRC2/N & $K_{S}$ & 600 & 40 & 40.0 & ADI & 16.2 & 9 \\
\hline & & 2013 May 2 & NIRC2/N & $K_{S}$ & 600 & 30 & 30.0 & ADI & 17.8 & 43 \\
\hline TYC 7443-1102-1 & J19560438-3207376 & 2012 May 22 & NIRC2/N & $K_{S}$ & 600 & 24 & 24.0 & ADI & 12.0 & 13 \\
\hline NLTT 48651 & J20043077-2342018 & 2011 Jun 21 & NIRC2/N & $H$ & 600 & 40 & 40.0 & ADI & 14.0 & 20 \\
\hline & & 2012 May 21 & NIRC2/N & $K_{S}$ & 600 & 8 & 4.0 & DI & $\ldots$ & $\ldots$ \\
\hline 2MASS J20284361-1128307 & J20284361-1128307 & 2012 May 10 & HiCIAO & $H$ & 300 & 18 & 18.0 & ADI & 8.6 & $\ldots$ \\
\hline & & 2013 Aug 17 & NIRC2/W & $K_{S}$ & 300 & 5 & 2.5 & ADI & 1.57 & $\ldots$ \\
\hline TYC 523-573-1 & $\mathrm{J} 20395460+0620118$ & 2012 Aug 23 & NIRC2/N & $H$ & 600 & 120 & 40.0 & ADI & 58.8 & 13 \\
\hline NLTT 50710 & $\mathrm{~J} 21111366-2248173$ & 2012 May 21 & NIRC2/N & $K_{S}$ & 600 & 28 & 28.0 & ADI & 10.6 & 16 \\
\hline GJ 4186 B & $\mathrm{J} 21160378+2951460$ & 2010 Aug 17 & NIRC2/N & $H$ & 600 & 30 & 30.0 & ADI & 14.3 & 26 \\
\hline & & 2011 Jun 21 & $\mathrm{NIRC} 2 / \mathrm{N}$ & $H$ & 600 & 40 & 40.0 & ADI & 15.1 & 24 \\
\hline
\end{tabular}


Table 3

(Continued)

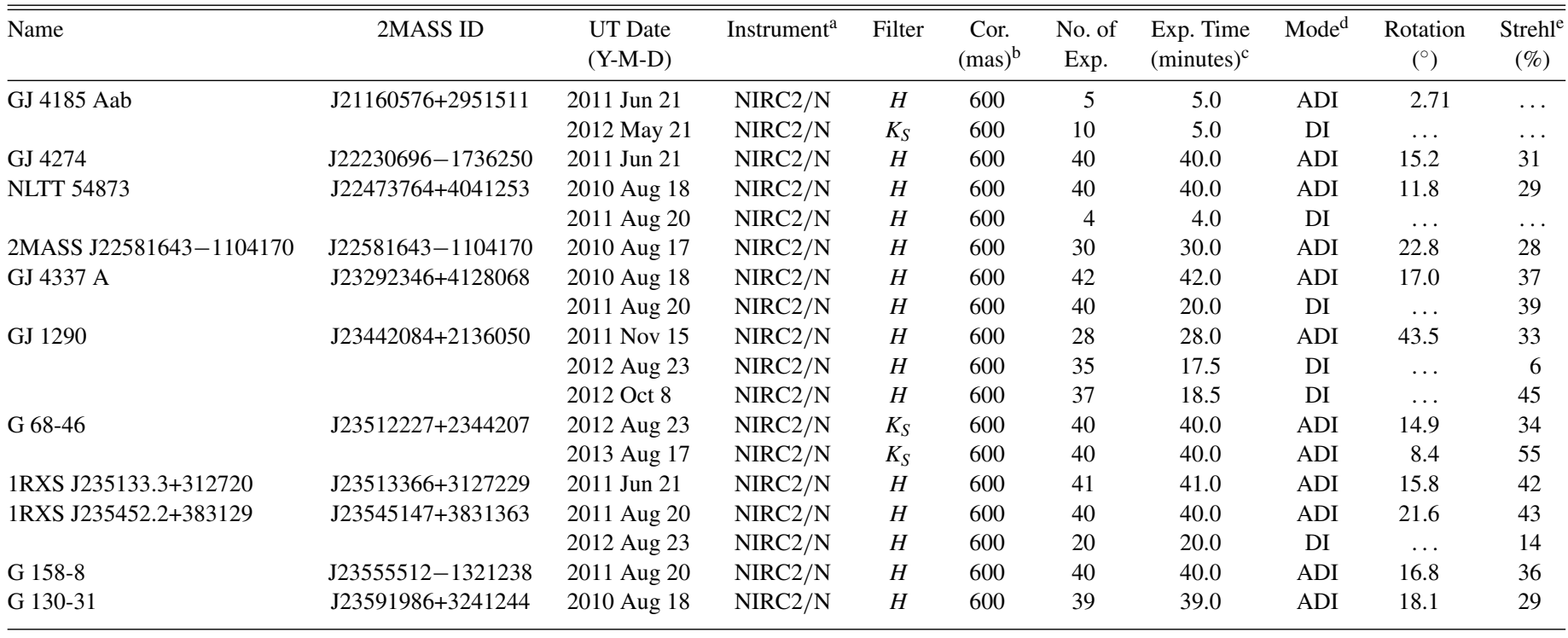

Notes.

a $\mathrm{NIRC} 2 / \mathrm{N}$ refers to the narrow $\left(\approx 10^{\prime \prime} \times 10^{\prime \prime}\right)$ camera mode. NIRC2/W refers to the wide $\left(\approx 40^{\prime \prime} \times 40^{\prime \prime}\right)$ camera.

b Angular diameter of coronagraph.

${ }^{c}$ Number of frames $\times$ coadds per frame $\times$ exposure time per frame. Most of the deep imaging consisted of 60 sec exposures with 1 coadd.

d ADI = Angular Differential Imaging; DI = Direct Imaging (rotator on).

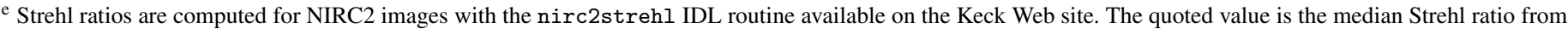
unsaturated images taken before and after deep ADI or DI sequences.

${ }^{\mathrm{f}}$ NLTT 13844 was observed with on-axis LGS-AO at Keck (Wizinowich et al. 2006; van Dam et al. 2006).

re-observe the stars with the worst data sets at Keck, a few of our observations only reach corresponding masses of $\sim 10-20 M_{\text {Jup }}$.

For HiCIAO, we adopt the following plate scale measurements, which we found slightly vary with wavelength (see Appendix A): $9.81 \pm 0.04$ mas pixel $^{-1}$ for $Y$ band, $9.75 \pm$ 0.04 mas pixel $^{-1}$ for $J$ band, $9.723 \pm 0.011$ mas pixel $^{-1}$ for $H$ band, and $9.67 \pm 0.03$ mas pixel $^{-1}$ for $K_{S}$ band. A constant plate scale orientation of $0.0 \pm 0.1$ is adopted for all of the filters. Post-fit residuals from the distortion solution are $\approx 1$ pixel.

\subsection{IRTF/SpeX Near-infrared Spectroscopy}

We obtained a near-infrared spectrum of the young, 5".6 separation substellar companion 2MASS J15594729+4403595 B (Section 5.1.4) with the Infrared Telescope Facility's (IRTF) SpeX spectrograph (Rayner et al. 2003) in short wavelength cross-dispersed (SXD) mode on 2012 August 11 UT in photometric conditions. A slit width of 0'.5 yielded a resolving power $(R \equiv \lambda / \Delta \lambda)$ of $\approx 1200$ from $0.8-2.5 \mu \mathrm{m}$. To avoid the host star, we oriented the slit perpendicular to the primary star-companion position angle (P.A.). We obtained a total of 36 minutes of integration time by nodding in an ABBA pattern along the slit. Immediately after, we observed the A0V standard 26 Ser at a similar airmass (1.22) for telluric correction. Flats and arc lamps were acquired at the same sky position. The spectra were extracted, median-combined, and telluric-corrected using Spextool reduction package (Vacca et al. 2003; Cushing et al. 2004). Table 4 summarizes our spectroscopic observations of three substellar companions in our sample.

\subsection{Keck/OSIRIS Near-infrared Spectroscopy}

On 2013 February 1 UT and 2013 February 2 UT, we observed GJ 3629 B and 1RXS J034231.8+121622 B with the
OH-Suppressing Infrared Imaging Spectrograph (OSIRIS; Larkin et al. 2006) at the Keck I telescope using NGS-AO (Table 4). Conditions were clear and both targets were observed at a low airmass of $\approx 1.1$. Since the companions are located $<1^{\prime \prime}$ from their host stars, we chose the 20 mas pixel ${ }^{-1}$ plate scale to finely sample the point-spread function (PSF) structure with a resolving power of $\approx 3800$. These observations benefited from a new grating installed in OSIRIS in 2012 December, increasing the average sensitivity by a factor of 1.83 compared to its previous performance on Keck II (Mieda et al. 2014).

We acquired $J b b$-, $H b b$-, and $K b b$-band spectra of GJ 3629 $B$ with the long axis of the 0 .'32 $\times 11^{\prime \prime} 28$ detector aligned with the primary-companion P.A. The close separation of this system $(0,2)$ ensured that both components were on the detector. The 1RXS J034231.8+121622 A-B separation is 0' 83 , so for this system we aligned the long-axis of the detector perpendicular to the primary-companion P.A. so that the primary fell off of the array. We nodded the telescope along the detector by $\approx 1^{\prime \prime}$ in an ABBA pattern for pair-wise sky subtraction. A0V standards were observed in each filter at a similar airmass following both science targets.

Basic image reduction, wavelength calibration, assemblage of the two-dimensional (2D) images into three-dimensional spectral cubes, and pair-wise sky subtraction was carried out using version 3.2 of the OSIRIS Data Reduction Pipeline with the latest rectification matrices from 2013 February. The companion and standard star spectra were then extracted from the cubes with aperture photometry. For GJ 3629 B we used aperture radii of 2 spaxels with an annulus of 2.5-4.0 spaxels to remove some contaminating flux from the nearby host star GJ 3629 A. No local sky subtraction was applied for 1RXS J034231.8+121622 
Table 4

Spectroscopic Observations

\begin{tabular}{|c|c|c|c|c|c|c|c|}
\hline Object & $\begin{array}{l}\text { Date } \\
\text { (UT) }\end{array}$ & $\begin{array}{l}\text { Telescope/ } \\
\text { Instrument }\end{array}$ & Filter & $\begin{array}{c}\text { Slit Width } \\
\left({ }^{\prime \prime}\right)\end{array}$ & $\begin{array}{c}\text { Plate Scale } \\
\left(\text { mas pixel }^{-1}\right)\end{array}$ & $\begin{array}{c}\text { Exp. Time } \\
\text { (minutes) }\end{array}$ & Standard \\
\hline 2MASS J15594729+4403595 B & 2012 Aug 11 & IRTF/SpeX-SXD & $\ldots$ & 0.5 & 150 & 36 & $26 \mathrm{Ser}$ \\
\hline \multirow[t]{3}{*}{ GJ $3629 \mathrm{~B}$} & 2013 Feb 1 & Keck I/OSIRIS & $J b b$ & $\ldots$ & 20 & 20 & HD 99960 \\
\hline & 2013 Feb 1 & Keck I/OSIRIS & $H b b$ & $\ldots$ & 20 & 13.5 & HD 99960 \\
\hline & 2013 Feb 1 & Keck I/OSIRIS & $K b b$ & $\ldots$ & 20 & 10 & HD 99960 \\
\hline \multirow[t]{2}{*}{ 1RXS J034231.8+121622 B } & 2013 Feb 2 & Keck I/OSIRIS & $H b b$ & $\ldots$ & 20 & 24 & HD 31411 \\
\hline & 2013 Feb 2 & Keck I/OSIRIS & $K b b$ & $\ldots$ & 20 & 24 & HD 31411 \\
\hline
\end{tabular}

B and the standards. The spectra were corrected for telluric absorption using the xtellcor_general routine in the IRTF Spextool reduction package (Vacca et al. 2003; Cushing et al. 2004). Finally, each band was flux calibrated using photometry from Bowler et al. (2012a) for GJ 3629 B and from Table 7 of this work for 1RXS J034231.8+121622 B.

\section{ADI PROCESSING PIPELINE}

We developed a processing pipeline for our ADI data to register the images, model and subtract the PSF and speckle pattern for each image, de-rotate and coadd the individual frames, identify point sources in the stacked images, compute contrast curves, and derive the sensitivity in mass and physical separation using information about the primary star coupled with evolutionary models. Below, we describe each step in detail for a typical ADI sequence consisting of forty $60 \mathrm{~s}$ coronagraphic images and short unsaturated frames.

\subsection{Image Registration}

For our NIRC2 images we correct for optical distortions using the narrow camera distortion solution made available by Keck Observatory (B. Cameron 2007, private communication), which yields post-fit residuals of $\sim 0.6$ mas in the $x$ and $y$ directions. For our HiCIAO data we derive distortion solutions from our own observations of the globular cluster M5 taken before and after the installation of a new camera lens in 2011 April. See Appendix A for details. The post-fit residuals across the entire array are $\sim 1$ pixel (10 mas).

Images are then registered and assembled into cubes. For NIRC2 we fit a 2D elliptical Gaussian to the star itself, which is visible behind the partly transparent coronagraph. For HiCIAO, which has an opaque coronagraph, we infer the position of the star by masking the coronagraph and fitting a 2D elliptical Gaussian to the PSF wings. Sky values are measured, stored, and subtracted from each image after masking the science target. Accurate accounting of the sky values are especially important for NIRC2. Since the coronagraph is not completely opaque, photometric calibration using the apparent brightness of the star behind the mask and the measured transmission of the coronagraph must also account for the background sky value. Once the stellar positions are measured, the images are assembled into a cube and aligned by shifting to a common position using sub-pixel resampling. Parallactic angles and north orientations on the detector are stored for later processing. For NIRC2, the parallactic angle is taken from FITS headers. For HiCIAO, it is computed from the hour angle at the time of the observation, target declination, and latitude of Mauna Kea.

\subsection{PSF and Speckle Subtraction}

The AO PSF comprises a mixture of static structure from the diffraction pattern and correlated, quasi-static speckle noise from imperfect wavefront correction and changing atmospheric conditions (Racine et al. 1999; Marois et al. 2000; Macintosh et al. 2005; Hinkley et al. 2007; Oppenheimer \& Hinkley 2009). Together these conspire to make the detection of faint point sources difficult in the contrast-limited regime, and removing these features requires modeling and subtracting the PSF pattern while minimizing the subtraction of actual companions. Observing strategies based on FOV rotation (Liu 2004; Marois et al. 2006) and/or chromatic dependencies of the PSF pattern (e.g., Sparks \& Ford 2002; Marois et al. 2005; Thatte et al. 2007; Biller et al. 2008; Crepp et al. 2011) together with more sophisticated processing techniques (Lafrenière et al. 2007b; Marois et al. 2010a; Soummer et al. 2011; Pueyo et al. 2012) yield contrasts $>14$ mag at $1^{\prime \prime}$ (e.g., Wahhaj et al. 2013b).

We experimented with a variety of PSF subtraction methods spanning a range of sky rotations and variable $\mathrm{AO}$ correction (caused both by seeing conditions and target brightness). Each method has advantages and disadvantages depending on the particular data set and instrument. For example, for small sky rotations, aggressive use of the Locally Optimized Combination of Images (LOCI) algorithm (Lafrenière et al. 2007b) results in substantial self-subtraction of real point sources. In these cases, simply subtracting a median-combined PSF model can result in a higher signal-to-noise ratio $(\mathrm{S} / \mathrm{N})$ for companions. In other cases where $\mathrm{AO}$ quality changes substantially during an ADI sequence, LOCI generally performs better than other simpler methods. We also tested a variation of LOCI described by Marois et al. (2010a) in which a small central portion of the region used to compute the correlation matrix is masked and then used to reconstruct the final image after calculation of the reference image weights. This method keeps noise levels across the processing region relatively constant and better preserves the photometry and astrometry of known point sources, but we found that it was not the best technique to identify real objects in a first (blind) pass.

Altogether we adopt three PSF subtraction methods to homogeneously process our survey data: subtraction of a scaled median-combined PSF model, a "conservative" application of LOCI algorithm, and an "aggressive" form of LOCI. Each technique is applied to the inner (contrast-limited) 3 " of our ADI sequences. In the background/read noise-limited regime beyond $3^{\prime \prime}$, we subtract a median-combined sky frame created from the data set itself.

For the scaled subtraction method we first create a PSF model by median-combining images in the ADI sequence. For each science image the model is then scaled to the annulus spanning the IWA out to $3^{\prime \prime}$ by computing the multiplicative factor $C$ that 


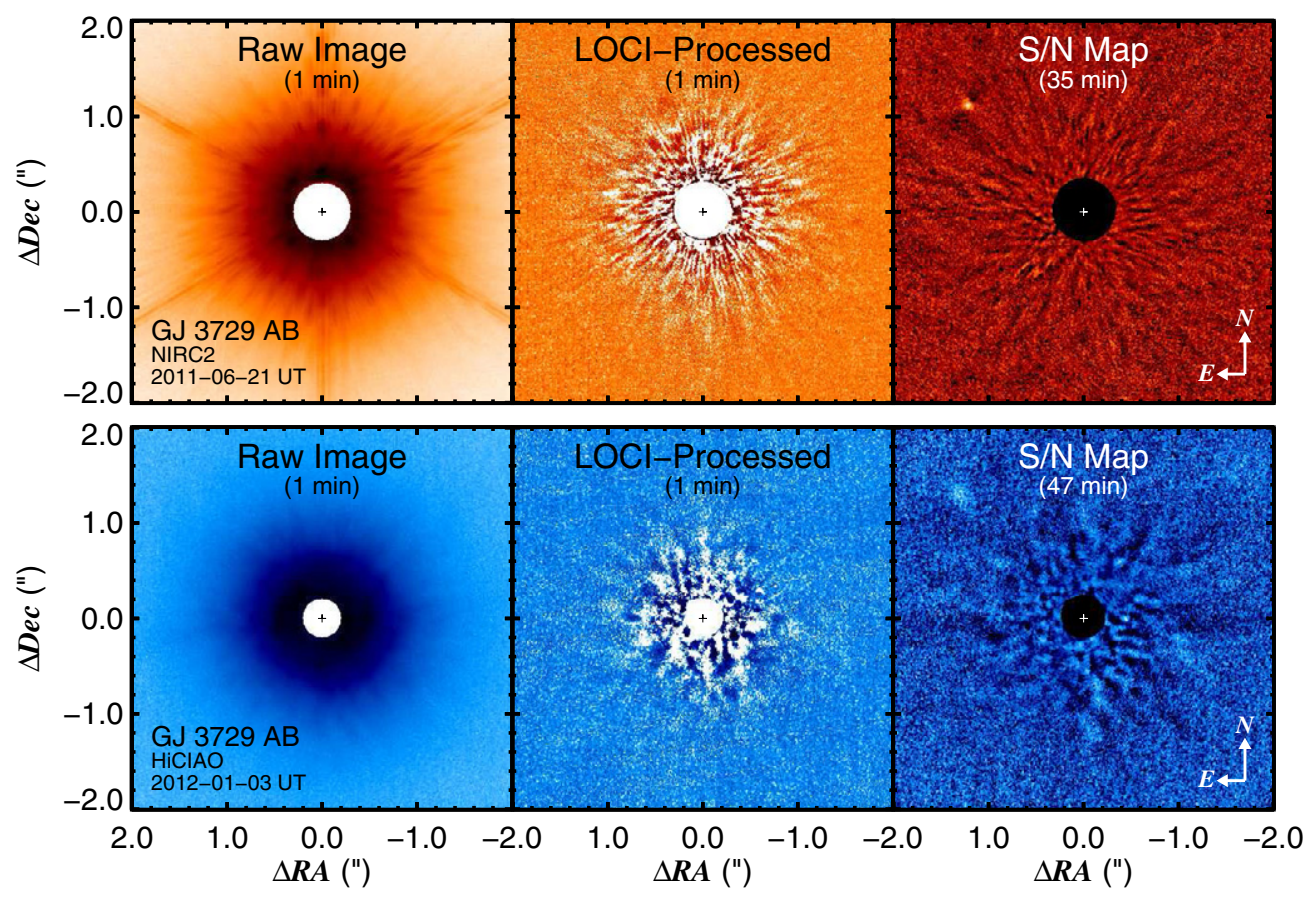

Figure 2. Example of ADI processing to reveal faint companions. The top panels show raw, LOCI-processed, and de-rotated and coadded images from NIRC2. A candidate companion is clearly visible $\sim 2^{\prime \prime}$ from the star GJ 3729 AB. The bottom panel shows the same sequence for observations with HiCIAO about six months later.

minimizes the $\chi^{2}$ value over all $n$ pixels:

$$
\begin{gathered}
\chi^{2}=\sum_{i=1}^{n}\left(\frac{f_{i}-C \mathcal{F}_{i}}{\sigma_{i}}\right)^{2}, \\
C=\frac{\sum f_{i} \mathcal{F}_{i} / \sigma_{i}^{2}}{\sum \mathcal{F}_{i}^{2} / \sigma_{i}^{2}} .
\end{gathered}
$$

Here $f_{i}$ and $\mathcal{F}_{i}$ are the flux at pixel $i$ in the science and model images in units of DN, and $\sigma_{i}$ is the uncertainty in the science flux. In this flux-limited regime, $\sigma_{i} \propto \sqrt{f}_{i}$, in which case $C$ simplifies to $\sum \mathcal{F}_{i} / \sum\left(\mathcal{F}_{i}^{2} / f_{i}\right)$.

Our implementation of LOCI follows the geometric regions described by Lafrenière et al. (2007b) with the following parameters: $N_{A}=300, g=1, d r=2$. We perform two reductions with minimum rotation parameter $N_{\delta}$ equal to 1.5 and 0.5 in units of PSF FWHM for the conservative and aggressive cases, respectively. If no reference frames satisfy the $N_{\delta}$ criterion for a particular annular subsection because of inadequate rotation at small separations then that section for that image is skipped. This affects some of the conservative LOCI processing at small separations near the IWA, but rarely influences the aggressive reduction.

Point sources bright enough to bias the reduction are masked out of the images prior to PSF subtraction. For the scaled median subtraction, masking reduces the influence of bright objects when computing scale factors. For the LOCI reduction, masking excludes these regions from the correlation matrix to avoid influencing the reference image weights.

\subsection{Point Source Identification}

After PSF subtraction, the individual images are de-rotated to a common P.A., median-combined, and oriented so that celestial north is at a P.A. of $0^{\circ}$. A map of the noise is created by computing the standard deviation of flux values in concentric annuli with a width of 3 pixels after rejecting outlier pixels with a clipping threshold of $4 \sigma$. In addition to outlier rejection, bright point sources are manually masked from the coadded image to minimize their influence on the noise measurements, and, from this, a signal-to-noise map is made to search for point sources in the images.

Automated point source identification is performed on the signal-to-noise maps using the max_search routine in the StarFinder AO imaging software package (Diolaiti et al. 2000). max_search identifies peak values above a threshold level relative to nearest neighbor pixel intensities. Low threshold values $(\sim 3-5)$ tend to produce a large number of false positives near the star where the speckle density is high, so we adopted a $7 \sigma$ limit. However, visual inspection of each image ultimately proved to be the most robust way to identify fainter point sources. In general, we found that artificial point sources injected into the median-combined images with peak values of $7 \sigma$ are reliably recovered from visual inspection across the entire image.

Figure 2 shows a typical reduction sequence with NIRC2 and HiCIAO. (This particular case is for the 50 mas binary GJ 3729 $\mathrm{AB}$, though it is not included in the final survey statistics because of its binarity.) The right-hand panels show the clear detection of a point source in the NIRC2 S/N maps, which is recovered in the HiCIAO data six months later. Multi-epoch astrometry shows the candidate companion is a background object.

\subsection{Contrast Curves}

Contrast curves are generated from the reduced images using the noise maps and the flux from the primary star. For NIRC2 we directly measure the star brightness behind the coronagraph. This allows us to monitor AO correction throughout the ADI sequences and calibrate the detection limits 
using the coronagraph throughput, which we have measured in the $H$ and $K_{S}$ filters using a binary star (Appendix B). An important caveat is that the background sky level must be taken into account since the apparent flux of the star behind the mask $\left(f_{m}\right)$ is the filter-dependent attenuation of the sky-plus-stellar flux, rather than simply the attenuated stellar flux alone. Since the sky level was subtracted from the raw images, it must also be taken into account to compute the corrected sky-subtracted stellar flux level $\left(f_{c}\right): f_{c}=\left(\left(f_{m}+s k y\right) / T_{\text {filt }}\right)-s k y$, where $T_{\text {filt }}$ is the filter-dependent transmission and $s k y$ is the background sky level. This is particularly important for our target sample of $\mathrm{M}$ dwarfs since the attenuated fluxes in $60 \mathrm{~s}$ exposures can be comparable to the (unattenuated) sky level in the raw frames. Finally, we use the median-corrected, sky-subtracted peak flux of the primary star $\left(f_{c}\right)$ from the sequence together with the noise map to compute contrast curves at the desired $\sigma$ level.

In a comprehensive analysis of deep ADI and SDI observations from the Gemini NICI Planet-Finding Campaign (Liu 2010a), Wahhaj et al. (2013a) show that the $5 \sigma$ threshold commonly used for contrast curves in high-contrast imaging surveys corresponds to $\approx 0.2 \mathrm{mag}$ brighter than the $95 \%$ recovery rate from Monte Carlo injections and extractions of artificial planets. For this survey we therefore adopt a $7 \sigma$ threshold as a robust threshold for our sensitivity limits.

The FOV coverage changes for each observation based on the instrument, coronagraph position on the detector, and total sky rotation of the ADI sequence. To make full use of the data (i.e., corners of the array), we also compute the fractional coverage as a function of radial separation from the star for each observation, which we incorporate into our sensitivity limits for the statistical analysis.

For our HiCIAO data we use the short unsaturated images taken in sets during the ADI sequences to photometrically calibrate the coronagraphic frames. For some Subaru data sets, neutral density filters with $\approx 1 \%$ and $\approx 10 \%$ throughputs were used to prevent saturating the detector. We adopt the following transmission measurements for the neutral density filters kindly provided by T. Kudo (2013, private communication): $9.740 \% \pm$ $0.022 \%$ for $N D 10$ in the $H$ band, $0.854 \% \pm 0.002 \%$ for $N D 1$ in the $H$ band, $10.460 \% \pm 0.020 \%$ for $N D 10$ in the $K_{S}$ band, and $1.142 \% \pm 0.026 \%$ for $N D 1$ in the $K_{S}$ band.

Unsaturated images are processed similarly to the coronagraphic frames: they are first registered, de-rotated to a common P.A. (which is necessary for multiple sets taken during an ADI sequence), median combined, and north aligned. $7 \sigma$ contrast curves are measured and joined with those from the deep imaging for separations inside the coronagraphic IWA.

Contrasts from the survey are shown in Figures 3-5 and listed in Table 5 . Beyond $\approx 2^{\prime \prime}$ the contrast curves flatten out to the background/read-noise level. The AO performance at both Keck and Subaru is sensitive to seeing conditions and rapidly degrades when seeing exceeds $\sim 1^{\prime \prime}$.5. Most of our observations with HiCIAO suffer from bad seeing, which is reflected in the modest contrast limits. Figure 6 summarizes the survey sensitivity: at $1^{\prime \prime}$, our NIRC2 contrasts reach 11.5-14 mag, but the HiCIAO observations span a larger range from 8-13 mag. The unsaturated frames reach between $\sim 3.5-5.5 \mathrm{mag}$ at 0.3 . In limiting apparent magnitude, our observations at $1^{\prime \prime}$ reach 15-23 mag, which translates to 13-23 mag in absolute magnitude. Note that we have excluded contrasts in these histograms for the two substellar companion host stars 1RXS J034231.8+121622 and 2MASS J15594729+4403595, for which we only obtained short images when we discovered the companions (Section 5.1).

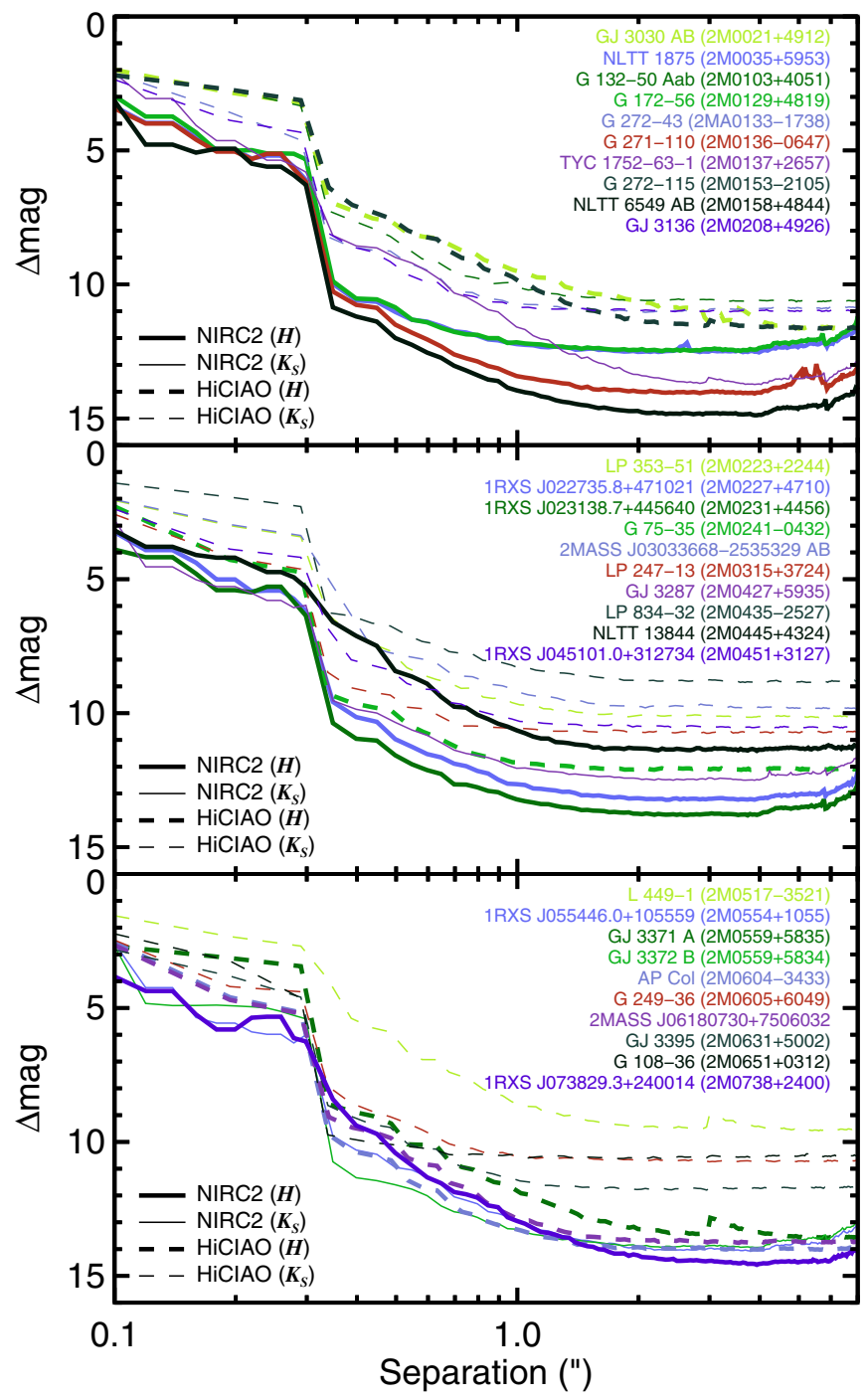

Figure 3. $7 \sigma$ contrast curves from our survey. Unsaturated frames are joined with the deep imaging at 0 '. 3 . PSF subtraction with LOCI is performed from $0.3-3^{\prime \prime}$ and scaled median subtraction is applied beyond $3^{\prime \prime}$.

These contrast curves are, however, included in our statistical analysis.

The right-most panel in Figure 6 shows the limiting mass at $1^{\prime \prime}$ as a function of physical separation at $1^{\prime \prime}$ for our entire sample. The median limiting mass is $5.5 M_{\text {Jup }}$ and we are sensitive to masses below $10 M_{\text {Jup }}$ at $1^{\prime \prime}$ for $85 \%$ of our targets. The median physical separation at $1^{\prime \prime}$ is $20.3 \mathrm{AU}$ and $85 \%$ of our targets correspond to physical separations less than 33 AU at $1^{\prime \prime}$.

\subsection{Astrometry and Photometry}

\subsubsection{Stellar Binaries}

Astrometry for bright companions from our short unsaturated images is computed in the following manner. After correcting each image for distortion, the separation and position angle are measured in one of two ways. For companions with small separations $\left(\lesssim 1^{\prime \prime}\right)$ we fit each binary component with a PSF composed of three elliptical Gaussians as described in Liu et al. (2008). This method performs well for both the NIRC2 and HiCIAO data in a variety of seeing conditions. For separations wide enough to avoid contamination from the primary $\left(\gtrsim 1^{\prime \prime}\right)$, 
Table 5

Contrast Curves

\begin{tabular}{|c|c|c|c|c|c|c|c|c|c|c|c|c|c|c|c|c|c|}
\hline \multirow[t]{2}{*}{ Name } & \multirow[t]{2}{*}{ UT Date } & \multirow[t]{2}{*}{ Inst. } & \multirow[t]{2}{*}{ Filter } & \multicolumn{14}{|c|}{ Contrast $(\Delta \mathrm{mag})$ [FOV Coverage] } \\
\hline & & & & $0 ! 1$ & $0 ! 2$ & 0.5 & $0 ! 75$ & $1^{\prime \prime}$ & $1^{\prime \prime} .5$ & $2^{\prime \prime}$ & $3^{\prime \prime}$ & $4^{\prime \prime}$ & $5^{\prime \prime}$ & $8^{\prime \prime}$ & $10^{\prime \prime}$ & $12^{\prime \prime}$ & $14^{\prime \prime}$ \\
\hline GJ $3030 \mathrm{AB}$ & 2011 Dec 27 & HiCIAO & $H$ & 1.9 & 2.8 & 7.6 & 8.7 & 9.5 & 10.3 & 10.6 & 11.4 & $11.3[1.0]$ & $11.6[1.0]$ & $11.7[1.0]$ & $11.3[0.9]$ & $10.8[0.5]$ & $10.4[0.1]$ \\
\hline LTT 1875 & 10 Aug 18 & NIRC2/N & $H$ & 3.3 & 5.0 & 11.0 & 11.8 & 12.2 & 12.4 & 12.5 & 12.5 & $12.5[1.0]$ & $12.3[0.7]$ & $11.6[0.1]$ & & & \\
\hline G 132-50 Aab & 11 Dec 28 & HiCIAO & $K_{S}$ & 2.2 & 2.8 & 8.2 & 9.7 & 10.1 & 10.5 & 10.6 & 10.6 & $10.6[1.0]$ & $10.6[1.0]$ & $10.6[1.0]$ & $10.4[0.9]$ & $9.8[0.6]$ & $9.4[0.1]$ \\
\hline G 172-56 & 10 Aug 17 & NIRC2/N & $H$ & 2.9 & 5.0 & 10.9 & 11.9 & 12.2 & 12.4 & 12.4 & 12.4 & $12.4[1.0]$ & $12.1[0.7]$ & $11.5[0.1]$ & & & \\
\hline G 272-43 & 2012 Jan 3 & HiCIAO & $K_{S}$ & 2.1 & 3.6 & 8.8 & 10.3 & 10.7 & 10.9 & 11.0 & 10.9 & $10.9[1.0]$ & $10.9[1.0]$ & $10.9[1.0]$ & $10.6[0.8]$ & $10.2[0.5]$ & $9.9[0.1]$ \\
\hline G 271-110 & 2010 Aug 18 & NIRC2/N & $H$ & 3.4 & 5.0 & 11.5 & 12.8 & 13.4 & 13.8 & 14.0 & 14.0 & $14.0[1.0]$ & $13.4[0.7]$ & $13.1[0.1]$ & $\ldots$ & $\ldots$ & $\ldots$ \\
\hline TYC 1752-63-1 & 2012 Aug 23 & $\mathrm{NIRC} 2 / \mathrm{N}$ & $K_{S}$ & 2.1 & 4.6 & 9.0 & 10.5 & 11.6 & 12.7 & 13.4 & 13.5 & $13.7[1.0]$ & $13.5[0.7]$ & $12.9[0.1]$ & $\ldots$ & $\ldots$ & $\ldots$ \\
\hline G 272-115 & 2011 Dec 27 & HiCIAO & $H$ & 2.2 & 2.7 & 7.5 & 9.0 & 9.9 & 11.0 & 11.3 & 11.3 & $11.6[1.0]$ & $11.6[1.0]$ & $11.6[1.0]$ & $11.4[0.8]$ & $11.1[0.4]$ & $10.8[0.1]$ \\
\hline NLTT 6549 AB & ag 20 & $\mathrm{NIRC} 2 / \mathrm{N}$ & $H$ & 3.1 & 4.9 & 12.0 & 13.2 & 14.0 & 14.5 & 14.7 & 14.8 & $14.9[1.0]$ & $14.6[0.7]$ & $13.8[0.1]$ & & & \\
\hline GJ 3136 & Jan 27 & HiCIAO & $K_{S}$ & 2.3 & 3.9 & 9.2 & 10.4 & 10.9 & 10.9 & 10.9 & 11.0 & $11.0[1.0]$ & $11.0[1.0]$ & $11.0[1.0]$ & $10.6[0.8]$ & $10.2[0.4]$ & $9.6[0.1]$ \\
\hline LP 353-51 & $\operatorname{Dec} 28$ & HiCIAO & $K_{S}$ & 2.0 & 3.0 & 7.7 & 9.1 & 9.7 & 10.0 & 10.1 & & $10.1[1.0]$ & $10.1[1.0]$ & $10.1[1.0]$ & $9.9[0.8]$ & $9.5[0.4]$ & $9.1[0.0]$ \\
\hline 1RXS J022735.8+471021 & ag 17 & $\mathrm{NIRC} 2 / \mathrm{N}$ & $H$ & 3.2 & 5.0 & 11.0 & 12.0 & 12.6 & 13.1 & 13.2 & 13.2 & $13.2[1.0]$ & $13.0[0.7]$ & $12.4[0.1]$ & $\ldots$ & $\ldots$ & $\ldots$ \\
\hline 1RXS J02 & 18 & $\mathrm{NIRC} 2 / \mathrm{N}$ & $H$ & & 5.4 & 11.6 & 12.7 & 13.2 & 13.6 & 3.8 & & 13.8[ & 13.5 & 12.8 & $\ldots$ & & $\ldots$ \\
\hline G 75-35 & 2012 Jan 3 & HiCIAO & $H$ & & 4.3 & 9.9 & 11.4 & 11.8 & 12.0 & 12.1 & 12.1 & 12.1[ & $12.1[1.0]$ & 12.1[ & $11.9[0.8]$ & $11.5[0.4]$ & $11.1[0.1]$ \\
\hline 2MASS J03033668-2535329 AB & 2011 Dec 28 & HiCIAO & $K_{S}$ & 0 & 3.0 & 7.7 & 8.6 & 9.2 & 9.6 & 9.8 & 9.7 & $9.8[1.0]$ & $9.8[1.0]$ & $9.8[1.0]$ & $9.7[0.8]$ & $9.4[0.3]$ & $9.1[0.0]$ \\
\hline LP 247-13 & ug 20 & $\mathrm{NIRC} 2 / \mathrm{N}$ & $H$ & 3.8 & 5 . & 8.9 & 10.6 & 11.6 & 13.0 & 13.4 & 13.5 & $13.7[1.0]$ & $13.7[0.6]$ & $13.8[0.0]$ & $\ldots$ & $\ldots$ & $\ldots$ \\
\hline GJ 3287 & 2013 Feb 4 & $\mathrm{NIRC} 2 / \mathrm{N}$ & $K_{S}$ & 2. & 5.3 & 10.3 & 11.5 & 12.1 & 12.3 & 12.4 & 12.4 & $12.5[1.0]$ & $12.3[0.7]$ & $11.6[0.1]$ & $\ldots$ & $\ldots$ & $\ldots$ \\
\hline LP 834-32 & 2011 Dec 28 & HiCIAO & $K_{S}$ & 1.4 & 1.9 & 6.7 & 7.8 & 8.3 & 8.6 & 8.8 & 8.8 & $8.8[1.0]$ & $8.8[1.0]$ & $8.8[1.0]$ & $8.5[0.8]$ & $8.2[0.4]$ & $7.9[0.1]$ \\
\hline NLTT 13844 & 012 Oct 8 & $\mathrm{NIRC} 2 / \mathrm{N}$ & $H$ & 3.2 & 4.2 & 8.4 & 9.8 & 10.6 & 11.2 & 11.3 & 11.3 & $11.4[1.0]$ & $11.3[0.6]$ & $11.3[0.0]$ & $\ldots$ & $\ldots$ & $\ldots$ \\
\hline 1RXS J045101.0+312734 & 2011 Jan 27 & HiCIAO & $K_{S}$ & 2.3 & 3.9 & 8.4 & 9.8 & 10.3 & 10.5 & 10.5 & 10.5 & $10.5[1.0]$ & $10.5[1.0]$ & $10.5[1.0]$ & $10.3[0.8]$ & $9.6[0.4]$ & $9.3[0.0]$ \\
\hline L 449-1 AB & 011 Dec 28 & HiCIAO & $K_{S}$ & 1.5 & 2.3 & 5.9 & 7.7 & 8.6 & 9.2 & 9.4 & 9.0 & $9.4[1.0]$ & $9.5[1.0]$ & $9.5[1.0]$ & $9.4[0.8]$ & $9.1[0.3]$ & $8.6[0.0]$ \\
\hline 1RXS J055446.0+105559 & 2013 Feb 4 & $\mathrm{NIRC} 2 / \mathrm{N}$ & $K_{S}$ & 2.5 & 5.6 & 10.9 & 12.2 & 12.9 & 13.7 & 14.0 & 14.0 & $14.1[1.0]$ & $13.7[0.7]$ & $13.1[0.1]$ & & & \\
\hline GJ 3371 A & 11 Dec 27 & HiCIAO & $H$ & 2.7 & 3.1 & 9.3 & 10.9 & 11.9 & 12.8 & 13.3 & 12.8 & $13.4[1.0]$ & $13.6[1.0]$ & $13.6[1.0]$ & $13.3[0.9]$ & $12.9[0.5]$ & $12.5[0.1]$ \\
\hline GJ 3372 B & 11 Mar 25 & IRC2/N & $K_{S}$ & 2.6 & 4.9 & 11.6 & 12.7 & 13.3 & 13.7 & 13.9 & 13.9 & $13.9[1.0]$ & $13.7[0.7]$ & $13.0[0.1]$ & 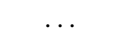 & $\ldots$ & $\ldots$ \\
\hline AP Col & $12 \operatorname{Jan} 3$ & CIAO & $H$ & 2.4 & 4.6 & 10.7 & 12.6 & 13.3 & 13.8 & 13.9 & 14.0 & $14.0[1.0]$ & $14.0[1.0]$ & $14.0[1.0]$ & $13.9[0.8]$ & $13.5[0.4]$ & $13.1[0.1]$ \\
\hline $249-36$ & $1 \mathrm{Jan} 27$ & CIAO & $K_{S}$ & 2.4 & 4.2. & 9 & 10.2 & 10.6 & 10.6 & 10.7 & 10.7 & $10.7[1.0]$ & $10.7[1.0]$ & $10.7[1.0]$ & 10.5[ & $9.9[0.4]$ & $9.5[0.0]$ \\
\hline S J06180730+7506032 & 12 Jan 3 & CIAO & $H$ & & 4.7 & 9.9 & 11.7 & 12.9 & 13.5 & 13.7 & 13.7 & $13.7[1.0]$ & $13.7[1.0]$ & $13.7[1.0]$ & $13.6[0.8]$ & $13.2[0.4]$ & $12.7[0.1]$ \\
\hline GJ 3395 & an 26 & ICIAO & $K_{S}$ & 2.8 & 3.8 & 9.5 & 11.0 & 11.5 & 11.7 & 11.8 & 11.7 & $11.7[1.0]$ & $11.7[1.0]$ & $11.7[1.0]$ & $11.4[0.8]$ & $10.9[0.5]$ & $10.3[0.1]$ \\
\hline G 108-36 & 2012 Jan 3 & HiCIAO & $K_{S}$ & 2.2 & 3.2 & 10.1 & 10.5 & 10.5 & 10.6 & 10.6 & 10.5 & $10.6[1.0]$ & $10.5[1.0]$ & $10.5[1.0]$ & $10.1[0.9]$ & $9.6[0.6]$ & $9.2[0.1]$ \\
\hline 1RXS J073829.3+240014 & 2010 Nov 27 & $\mathrm{NIRC} 2 / \mathrm{N}$ & $H$ & 3.8 & 5.8 & 10.4 & 12.0 & 12.9 & 13.9 & 14.2 & 14.4 & $14.6[1.0]$ & $14.5[0.7]$ & $13.8[0.0]$ & $\ldots$ & $\ldots$ & $\ldots$ \\
\hline 1RXS J091744.5+461229 AB & 2011 Dec 28 & HiCIAO & $K_{S}$ & 0.7 & 2.1 & 7.6 & 8.8 & 9.4 & 9.8 & 9.9 & 9.8 & $10.0[1.0]$ & $9.9[1.0]$ & $9.9[1.0]$ & $9.7[0.8]$ & $9.2[0.4]$ & $8.8[0.1]$ \\
\hline GJ $354.1 \mathrm{~B}$ & 2013 May 2 & $\mathrm{NIRC} 2 / \mathrm{N}$ & $K_{S}$ & 2.3 & 5.0 & 10.4 & 11.4 & 11.8 & 12.1 & 12.2 & 12.2 & $12.2[1.0]$ & $11.9[0.7]$ & $11.2[0.1]$ & $\ldots$ & $\ldots$ & $\ldots$ \\
\hline PYC 09362+3731 AB & 2012 May 22 & $\mathrm{NIRC} 2 / \mathrm{N}$ & $H$ & 2.9 & 4.2 & 11.8 & 12.8 & 13.7 & 14.4 & 14.7 & 14.6 & $14.9[1.0]$ & $14.6[0.7]$ & $13.9[0.1]$ & $\cdots$ & $\ldots$ & $\ldots$ \\
\hline G 161-71 & 2011 Nov 16 & $\mathrm{NIRC} 2 / \mathrm{N}$ & $H$ & 3.2 & 4.2 & 11.1 & 12.3 & 13.3 & 14.0 & 14.3 & 13.0 & $13.5[1.0]$ & $13.1[0.9]$ & $13.1[0.1]$ & $\cdots$ & $\ldots$ & $\ldots$ \\
\hline NLTT $22741 \mathrm{~A}$ & 2012 May 21 & $\mathrm{NIRC} 2 / \mathrm{N}$ & $K_{S}$ & 2.0 & 3.5 & 8.9 & 9.7 & 10.1 & 10.5 & 10.6 & 10.6 & $10.6[1.0]$ & $10.5[0.7]$ & $9.8[0.0]$ & $\cdots$ & $\ldots$ & $\ldots$ \\
\hline GJ $3577 \mathrm{~A}$ & 2013 Feb 4 & $\mathrm{NIRC} 2 / \mathrm{N}$ & $K_{S}$ & 2.3 & 4.9 & 10.7 & 11.8 & 12.4 & 12.8 & 13.0 & 12.9 & $13.0[1.0]$ & $12.6[0.8]$ & $12.0[0.1]$ & $\ldots$ & $\ldots$ & $\ldots$ \\
\hline GJ 3578 B & $2013 \mathrm{Feb} 4$ & NIRC2/N & $K_{S}$ & 2.5 & 5.1 & 11.4 & 12.3 & 12.7 & 12.9 & 13.0 & 13.0 & $13.0[1.0]$ & $12.7[0.8]$ & $11.9[0.1]$ & $\ldots$ & $\ldots$ & $\ldots$ \\
\hline G 196-3 A & 2011 Jan 26 & & $K_{S}$ & 2.2 & 3.5 & 8.6 & 10.5 & 11.2 & 11.5 & 11.5 & 11.5 & $11.5[1.0]$ & $11.5[1.0]$ & $11.5[1.0]$ & $11.3[0.7]$ & $10.9[0.3]$ & $10.5[0.0]$ \\
\hline GJ $2079 \mathrm{AB}$ & 2013 Feb 4 & NIRC2/N & $K_{S}$ & 1.5 & 4.7 & 9.4 & 11.0 & 12.1 & 13.2 & 13.8 & 13.1 & & $13.3[0.7]$ & $12.7[0.1]$ & & & \\
\hline GJ 3629 AB & 2012 May 10 & HiCIAO & $H$ & 2.4 & 4.3 & 9.0 & 10.3 & 10.8 & 11.0 & 11.1 & 11.1 & $11.1[1.0]$ & $11.1[1.0]$ & $11.1[1.0]$ & $10.9[0.8]$ & $10.3[0.4]$ & $9.9[0.1]$ \\
\hline GJ 3639 & 2011 Jan 27 & HiCIAO & $K_{S}$ & 3.0 & 4.3 & 10.4 & 10.7 & 10.7 & 10.7 & 10.8 & 10.8 & $10.7[1.0]$ & $10.7[1.0]$ & $10.8[1.0]$ & $10.3[0.9]$ & $9.6[0.6]$ & $8.8[0.1]$ \\
\hline NLTT 26114 & 2012 Jan 14 & $\mathrm{NIRC} 2 / \mathrm{N}$ & $K$ & 0.0 & 9.6 & 10.0 & 11.6 & 12.2 & 12.6 & 12.8 & 12.7 & $12.8[1.0]$ & $12.6[0.7]$ & $11.8[0.1]$ & & $\ldots$ & $\ldots$ \\
\hline NLTT 26359 & 2011 Dec 27 & HiCIAO & $K_{S}$ & 2.4 & 4.0 & 9.6 & 11.5 & 12.1 & 12.5 & 12.7 & 12.6 & $12.7[1.0]$ & $12.7[1.0]$ & $12.6[1.0]$ & $12.4[0.8]$ & $12.0[0.4]$ & $11.6[0.1]$ \\
\hline G 119-62 & 2011 Jan 26 & HiCIAO & $K_{S}$ & 2.6 & 4.4 & 9.3 & 11.0 & 11.4 & 11.6 & 11.7 & 11.7 & $11.6[1.0]$ & $11.7[1.0]$ & $11.6[1.0]$ & $11.3[0.8]$ & $10.9[0.4]$ & $10.4[0.1]$ \\
\hline 2MASS J11240434+3808108 & 2011 Mar 25 & $\mathrm{NIRC} 2 / \mathrm{N}$ & $K_{S}$ & 2.7 & 5.3 & 11.3 & 12.4 & 12.9 & 13.2 & 13.2 & 13.2 & $13.3[1.0]$ & $13.1[0.7]$ & $12.3[0.1]$ & & & \\
\hline TWA 30 A & 2011 Mar 25 & NIRC2/N & $K_{S}$ & 2.1 & 3.8 & 9.1 & 10.1 & 10.6 & 10.8 & 10.9 & 10.9 & $11.0[1.0]$ & $10.8[0.7]$ & $10.2[0.0]$ & & & \\
\hline
\end{tabular}


Table 5

\begin{tabular}{|c|c|c|c|c|c|c|c|c|c|c|c|c|c|c|c|c|c|}
\hline \multirow[t]{2}{*}{ Name } & \multirow[t]{2}{*}{ UT Date } & \multirow[t]{2}{*}{ Inst. } & \multirow[t]{2}{*}{ Filter } & \multicolumn{14}{|c|}{ Contrast ( $\Delta \mathrm{mag})$ [FOV Coverage] } \\
\hline & & & & 0.1 & 0.2 & 0.5 & $0 ! 75$ & $1^{\prime \prime}$ & 1..5 & $2^{\prime \prime}$ & $3^{\prime \prime}$ & $4^{\prime \prime}$ & $5^{\prime \prime}$ & $8^{\prime \prime}$ & $10^{\prime \prime}$ & $12^{\prime \prime}$ & $14^{\prime \prime}$ \\
\hline G 10-52 & 2011 Jan 27 & HiCIAO & $K_{S}$ & 2.7 & 4.3 & 9.8 & 10.7 & 10.8 & 10.8 & 10.9 & 10.9 & $10.9[1.0]$ & $10.9[1.0]$ & $10.9[1.0]$ & $10.6[0.8]$ & $10.0[0.3]$ & $9.7[0.0]$ \\
\hline LP 734-34 & 2011 Dec 27 & HiCIAO & $K_{S}$ & 2.3 & 3.9 & 9.1 & 10.1 & 10.2 & 10.4 & 10.4 & 10.4 & $10.4[1.0]$ & $10.4[1.0]$ & $10.4[1.0]$ & $10.1[0.8]$ & $9.7[0.4]$ & $9.3[0.1]$ \\
\hline G $13-33 \mathrm{AB}$ & 2012 May 21 & $\mathrm{NIRC} 2 / \mathrm{N}$ & $K_{S}$ & 1.9 & 3.4 & 9.3 & 10.2 & 10.8 & 11.2 & 11.4 & 11.4 & $11.5[1.0]$ & $11.3[0.7]$ & $10.6[0.0]$ & $\ldots$ & $\ldots$ & $\ldots$ \\
\hline GJ $3729 \mathrm{AB}$ & 2011 Jun 21 & $\mathrm{NIRC} 2 / \mathrm{N}$ & $H$ & 2.9 & 4.8 & 11.1 & 12.3 & 13.1 & 13.8 & 14.1 & 14.1 & $14.2[1.0]$ & $13.9[0.7]$ & $13.3[0.1]$ & $\ldots$ & $\ldots$ & $\ldots$ \\
\hline 1RXS J124147.5+564506 & 2011 Jan 26 & HiCIAO & $K_{S}$ & 2.5 & 3.5 & 8.5 & 9.5 & 9.6 & 9.7 & 9.8 & 9.7 & $9.7[1.0]$ & $9.7[1.0]$ & $9.7[1.0]$ & $9.5[0.8]$ & $9.0[0.5]$ & $8.5[0.1]$ \\
\hline LHS 2613 & 2012 Jan 3 & HiCIAO & $H$ & 2.5 & 4.7 & 9.7 & 11.5 & 12.4 & 13.0 & 13.2 & 13.2 & $13.2[1.0]$ & $13.3[1.0]$ & $13.2[1.0]$ & $13.0[0.8]$ & $12.5[0.5]$ & $12.1[0.1]$ \\
\hline LHS 2672 & 2012 May 10 & HiCIAO & $H$ & 2.5 & 4.7 & 10.5 & 11.8 & 12.4 & 12.5 & 12.6 & 12.5 & $12.6[1.0]$ & $12.6[1.0]$ & $12.6[1.0]$ & $12.3[0.8]$ & $11.6[0.5]$ & $11.3[0.1]$ \\
\hline GJ $1167 \mathrm{~A}$ & 2011 Jan 27 & HiCIAO & $K_{S}$ & 2.7 & 4.0 & 10.2 & 10.7 & 10.7 & 10.8 & 10.8 & 10.8 & $10.7[1.0]$ & $10.8[1.0]$ & $10.8[1.0]$ & $10.4[0.8]$ & $9.8[0.4]$ & $9.3[0.1]$ \\
\hline LHS 2686 & 2011 Jun 21 & $\mathrm{NIRC} 2 / \mathrm{N}$ & $H$ & 4.1 & 5.0 & 11.3 & 12.4 & 13.3 & 13.9 & 14.1 & 14.2 & $14.2[1.0]$ & $14.0[0.7]$ & $13.3[0.1]$ & $\ldots$ & $\ldots$ & $\ldots$ \\
\hline 2MASS J13233804-2554449 & $2013 \mathrm{Feb} 4$ & $\mathrm{NIRC} 2 / \mathrm{N}$ & $K_{S}$ & 2.5 & 5.1 & 9.9 & 11.0 & 12.0 & 12.9 & 13.3 & 13.1 & $13.4[1.0]$ & $13.0[0.7]$ & $12.5[0.1]$ & & & $\ldots$ \\
\hline 2MASS J13292408-1422122 & 2011 Mar 25 & $\mathrm{NIRC} 2 / \mathrm{N}$ & $K_{S}$ & 2.7 & 5.4 & 10.8 & 12.4 & 13.1 & 13.7 & 13.9 & 13.9 & $14.0[1.0]$ & $13.8[0.7]$ & $13.1[0.1]$ & $\cdots$ & $\cdots$ & $\ldots$ \\
\hline 2MASS J14124864-1629561 & 2013 May 2 & $\mathrm{NIRC} 2 / \mathrm{N}$ & $K_{S}$ & 2.4 & 4.9 & 10.6 & 11.8 & 12.5 & 13.1 & 13.2 & 13.2 & $13.3[1.0]$ & $13.0[0.7]$ & $12.4[0.1]$ & $\ldots$ & $\ldots$ & $\ldots$ \\
\hline GQ Vir & 2012 May 21 & $\mathrm{NIRC} 2 / \mathrm{N}$ & $K_{S}$ & 2.0 & 4.3 & 10.1 & 11.0 & 11.7 & 12.3 & 12.6 & 12.6 & $12.7[1.0]$ & $12.5[0.7]$ & $11.8[0.1]$ & $\cdots$ & $\ldots$ & $\ldots$ \\
\hline 2MASS J14442809-0424078 & 2011 Mar 25 & $\mathrm{NIRC} 2 / \mathrm{N}$ & $K_{S}$ & 2.5 & 5.2 & 10.9 & 11.9 & 12.6 & 13.0 & 13.1 & 13.1 & $13.1[1.0]$ & $13.0[0.7]$ & $12.5[0.0]$ & $\ldots$ & $\ldots$ & $\ldots$ \\
\hline G $166-49$ & 2012 May 22 & $\mathrm{NIRC} 2 / \mathrm{N}$ & $H$ & 3.5 & 4.9 & 11.7 & 12.6 & 13.2 & 13.9 & 14.2 & 13.8 & $13.6[1.0]$ & $13.5[0.9]$ & $13.0[0.1]$ & $\ldots$ & $\ldots$ & $\ldots$ \\
\hline 1RXS J150907.2+590422 A & 2012 May 21 & $\mathrm{NIRC} 2 / \mathrm{N}$ & $K_{S}$ & 2.2 & 4.7 & 9.9 & 10.9 & 11.6 & 12.3 & 12.5 & 12.5 & $12.6[1.0]$ & $12.4[0.7]$ & $11.7[0.1]$ & $\ldots$ & $\ldots$ & $\ldots$ \\
\hline 2MASS J15323737+4653048 & 2013 Aug 17 & $\mathrm{NIRC} 2 / \mathrm{N}$ & $K_{S}$ & 2.5 & 5.1 & 10.6 & 12.0 & 13.0 & 13.8 & 14.1 & 14.2 & $14.3[1.0]$ & $14.2[0.7]$ & $13.5[0.1]$ & $\ldots$ & $\ldots$ & $\ldots$ \\
\hline NLTT 40561 & 2010 Aug 18 & $\mathrm{NIRC} 2 / \mathrm{N}$ & $H$ & 3.6 & 5.2 & 9.6 & 11.3 & 12.0 & 12.6 & 12.9 & 13.0 & $13.0[1.0]$ & $12.9[0.7]$ & $12.4[0.0]$ & $\ldots$ & $\ldots$ & $\ldots$ \\
\hline G 202-48 & 2011 Jun 21 & $\mathrm{NIRC} 2 / \mathrm{N}$ & $H$ & 3.6 & 5.0 & 10.8 & 12.1 & 13.4 & 14.9 & 15.7 & 15.7 & $16.2[1.0]$ & $16.0[0.7]$ & $15.5[0.0]$ & $\ldots$ & $\ldots$ & $\ldots$ \\
\hline GJ 669 B & 2010 Aug 18 & $\mathrm{NIRC} 2 / \mathrm{N}$ & $H$ & 3.8 & 5.3 & 10.9 & 12.6 & 13.6 & 14.3 & 14.2 & 14.6 & $14.6[1.0]$ & $14.4[0.7]$ & $13.7[0.1]$ & $\ldots$ & $\ldots$ & $\ldots$ \\
\hline GJ 669 A & 2012 May 10 & HiCIAO & $H$ & 2.4 & 4.5 & 10.0 & 11.8 & 12.9 & 13.6 & 13.8 & 13.9 & $13.9[1.0]$ & $13.9[1.0]$ & $13.9[1.0]$ & $13.6[0.8]$ & $13.0[0.4]$ & $12.9[0.1]$ \\
\hline LHS 3321 & 2011 Jun 21 & $\mathrm{NIRC} 2 / \mathrm{N}$ & $H$ & 4.2 & 5.4 & 11.9 & 13.2 & 14.4 & 15.5 & 16.1 & 15.6 & $16.2[1.0]$ & $15.6[0.8]$ & $15.1[0.1]$ & $\ldots$ & $\ldots$ & $\ldots$ \\
\hline G $227-22$ & 2010 Aug 17 & $\mathrm{NIRC} 2 / \mathrm{N}$ & $H$ & 3.0 & 4.9 & 10.6 & 12.0 & 13.0 & 13.9 & 14.3 & 14.4 & $14.4[1.0]$ & $14.3[0.7]$ & $13.6[0.1]$ & $\ldots$ & $\ldots$ & $\ldots$ \\
\hline LP $390-16$ & 2011 Mar 25 & $\mathrm{NIRC} 2 / \mathrm{N}$ & $K_{S}$ & 2.9 & 5.3 & 11.1 & 12.2 & 13.0 & 13.7 & 13.9 & 13.8 & $13.8[1.0]$ & $13.2[0.8]$ & $12.9[0.1]$ & $\ldots$ & $\ldots$ & $\ldots$ \\
\hline GJ $9652 \mathrm{~A}$ & 2011 Aug 20 & $\mathrm{NIRC} 2 / \mathrm{N}$ & $H$ & 3.3 & 4.5 & 10.5 & 11.9 & 12.8 & 14.0 & 14.7 & 15.3 & $15.5[1.0]$ & $15.4[0.6]$ & $15.5[0.0]$ & $\ldots$ & $\ldots$ & $\ldots$ \\
\hline 2MASS J19303829-1335083 & 2011 Jul 1 & $\mathrm{NIRC} 2 / \mathrm{N}$ & $H$ & 3.0 & 3.7 & 10.6 & 11.4 & 11.7 & 11.8 & 11.8 & 11.9 & $11.9[1.0]$ & $11.7[0.7]$ & $10.8[0.1]$ & $\ldots$ & $\ldots$ & $\ldots$ \\
\hline 1RXS J193528.9+374605 & 2011 Aug 20 & $\mathrm{NIRC} 2 / \mathrm{N}$ & $H$ & 4.2 & 5.2 & 11.5 & 12.8 & 14.0 & 15.0 & 15.4 & 15.5 & $15.8[1.0]$ & $15.6[0.8]$ & $14.8[0.1]$ & $\ldots$ & $\ldots$ & $\ldots$ \\
\hline 2MASS J19435432-0546363 & 2012 May 21 & $\mathrm{NIRC} 2 / \mathrm{N}$ & $K_{S}$ & 1.8 & 3.4 & 8.9 & 9.9 & 10.5 & 11.1 & 11.3 & 11.3 & $11.4[1.0]$ & $11.2[0.7]$ & $10.5[0.1]$ & $\ldots$ & $\ldots$ & $\ldots$ \\
\hline TYC 7443-1102-1 & 2012 May 22 & $\mathrm{NIRC} 2 / \mathrm{N}$ & $K_{S}$ & 1.8 & 3.8 & 8.6 & 9.7 & 10.6 & 11.4 & 11.8 & 11.6 & $12.0[1.0]$ & $11.9[0.7]$ & $11.3[0.1]$ & $\ldots$ & $\ldots$ & $\ldots$ \\
\hline NLTT 48651 & 2011 Jun 21 & $\mathrm{NIRC} 2 / \mathrm{N}$ & $H$ & 3.1 & 4.4 & 10.8 & 12.1 & 13.2 & 14.1 & 14.4 & 14.5 & $14.7[1.0]$ & $14.5[0.7]$ & $13.9[0.1]$ & $\ldots$ & $\ldots$ & $\ldots$ \\
\hline 2MASS J20284361-1128307 & 2012 May 10 & HiCIAO & $H$ & 2.2 & 4.3 & 8.5 & 10.7 & 11.8 & 12.3 & 12.5 & 12.4 & $12.4[1.0]$ & $12.4[1.0]$ & $12.5[1.0]$ & $12.3[0.8]$ & $11.8[0.3]$ & $11.5[0.0]$ \\
\hline TYC 523-573-1 & 2012 Aug 23 & $\mathrm{NIRC} 2 / \mathrm{N}$ & $H$ & 2.4 & 4.2 & 10.6 & 11.7 & 12.9 & 13.9 & 14.4 & 13.3 & $14.2[1.0]$ & $14.0[0.9]$ & $13.4[0.2]$ & $\ldots$ & $\ldots$ & $\ldots$ \\
\hline NLTT 50710 & 2012 May 21 & $\mathrm{NIRC} 2 / \mathrm{N}$ & $K_{S}$ & 2.0 & 3.8 & 8.6 & 9.4 & 10.2 & 10.7 & 10.9 & 10.9 & $11.0[1.0]$ & $10.9[0.7]$ & $10.2[0.0]$ & $\ldots$ & $\ldots$ & $\ldots$ \\
\hline GJ 4186 B & 2011 Jun 21 & $\mathrm{NIRC} 2 / \mathrm{N}$ & $H$ & 3.7 & 4.7 & 10.8 & 12.2 & 13.1 & 13.8 & 14.1 & 14.1 & $14.2[1.0]$ & $14.0[0.7]$ & $13.3[0.1]$ & $\ldots$ & $\ldots$ & $\ldots$ \\
\hline GJ 4274 & 2011 Jun 21 & $\mathrm{NIRC} 2 / \mathrm{N}$ & $H$ & 3.8 & 4.9 & 11.2 & 12.8 & 13.8 & 14.7 & 15.1 & 15.0 & $15.3[1.0]$ & $15.1[0.7]$ & $14.4[0.1]$ & $\ldots$ & $\ldots$ & $\ldots$ \\
\hline NLTT 54873 & 2010 Aug 18 & $\mathrm{NIRC} 2 / \mathrm{N}$ & $H$ & 3.5 & 5.1 & 11.0 & 12.1 & 12.7 & 13.1 & 13.2 & 13.2 & $13.2[1.0]$ & $13.1[0.7]$ & $12.3[0.0]$ & $\ldots$ & $\ldots$ & $\ldots$ \\
\hline 2MASS J22581643-1104170 & 2010 Aug 17 & $\mathrm{NIRC} 2 / \mathrm{N}$ & $H$ & 3.2 & 5.0 & 10.5 & 12.0 & 13.0 & 13.7 & 14.0 & 14.1 & $14.1[1.0]$ & $13.9[0.7]$ & $13.3[0.1]$ & $\ldots$ & $\ldots$ & $\ldots$ \\
\hline GJ 4337 A & 2010 Aug 18 & $\mathrm{NIRC} 2 / \mathrm{N}$ & $H$ & 3.8 & 5.8 & 11.6 & 13.0 & 13.8 & 14.5 & 14.6 & 14.6 & $14.6[1.0]$ & $14.4[0.7]$ & $13.6[0.1]$ & $\ldots$ & $\ldots$ & $\ldots$ \\
\hline GJ 1290 & 2011 Nov 15 & $\mathrm{NIRC} 2 / \mathrm{N}$ & $H$ & 3.8 & 4.6 & 11.5 & 12.9 & 13.8 & 14.5 & 14.8 & 14.7 & $14.9[1.0]$ & $14.3[0.9]$ & $13.4[0.1]$ & $\ldots$ & $\ldots$ & $\ldots$ \\
\hline G 68-46 & 2013 Aug 17 & $\mathrm{NIRC} 2 / \mathrm{N}$ & $K_{S}$ & 2.5 & 5.2 & 10.4 & 11.9 & 12.6 & 13.1 & 13.3 & 13.3 & $13.4[1.0]$ & $13.3[0.7]$ & $12.8[0.0]$ & $\ldots$ & $\ldots$ & $\ldots$ \\
\hline 1RXS J235133.3+312720 & 2011 Jun 21 & $\mathrm{NIRC} 2 / \mathrm{N}$ & $H$ & 3.7 & 5.5 & 10.4 & 11.7 & 12.9 & 13.7 & 14.0 & 14.1 & $14.2[1.0]$ & $14.0[0.7]$ & $13.2[0.1]$ & $\cdots$ & $\cdots$ & $\ldots$ \\
\hline 1RXS J235452.2+383129 & 2011 Aug 20 & $\mathrm{NIRC} 2 / \mathrm{N}$ & $H$ & 4.5 & 5.5 & 11.7 & 13.0 & 13.8 & 14.5 & 14.7 & 14.8 & $14.9[1.0]$ & $14.6[0.7]$ & $14.0[0.1]$ & $\ldots$ & $\ldots$ & $\ldots$ \\
\hline G $158-8$ & 2011 Aug 20 & $\mathrm{NIRC} 2 / \mathrm{N}$ & $H$ & 3.9 & 4.8 & 11.4 & 12.8 & 13.6 & 14.2 & 14.4 & 14.4 & $14.5[1.0]$ & $14.3[0.7]$ & $13.6[0.1]$ & $\ldots$ & $\ldots$ & $\ldots$ \\
\hline G 130-31 & 2010 Aug 18 & $\mathrm{NIRC} 2 / \mathrm{N}$ & $H$ & 3.9 & 5.5 & 11.3 & 12.2 & 12.8 & 13.1 & 13.2 & 13.2 & $13.2[1.0]$ & $13.0[0.7]$ & $12.3[0.1]$ & $\ldots$ & $\ldots$ & $\ldots$ \\
\hline Median NIRC2 Contrast & $\ldots$ & $\mathrm{NIRC} 2 / \mathrm{N}$ & $\ldots$ & 3.1 & 5.1 & 10.8 & 12.0 & 12.9 & 13.6 & 13.8 & 13.5 & 13.7 & 13.3 & 12.9 & & & \\
\hline Median HiCIAO Contrast & $\ldots$ & HiCIAO & $\ldots$ & 2.4 & 3.9 & 9.2 & 10.5 & 10.7 & 10.9 & 11.0 & 11.0 & 11.0 & 11.0 & 11.0 & 10.6 & 10.2 & 9.9 \\
\hline
\end{tabular}




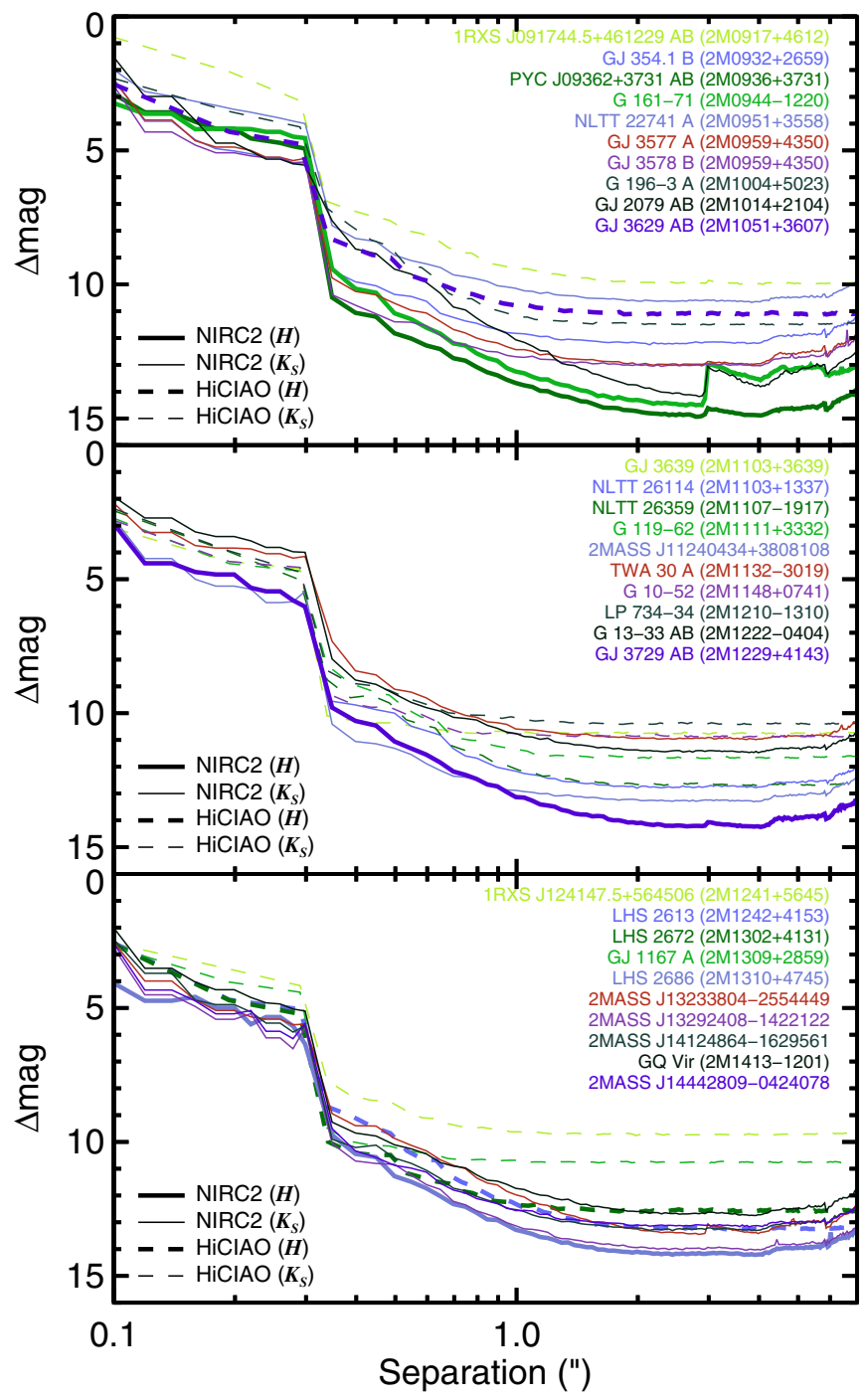

Figure 4. $7 \sigma$ contrast curves from our survey (continued).

we use aperture photometry after subtracting the sky level from each image. The mean of the separation and P.A. measured from individual images are adopted for our astrometry.

Several sources contribute to the uncertainty in these values: random sub-pixel centroid measurement errors from image to image, systematic effects from residuals in the distortion solution, uncertainties in the absolute sky orientation on the detector, and the finite precision of the measured plate scale. These independent errors are propagated analytically to produce our final astrometric uncertainties. The separation $\rho$ is therefore

$$
\begin{gathered}
\rho=s \bar{\rho}_{\text {meas }} \pm s \bar{\rho}_{\text {meas }}\left(\left(\frac{\sigma_{s}}{s}\right)^{2}+\left(\frac{\sigma_{\bar{\rho}, \text { tot }}}{\sigma_{\bar{\rho}, \text { meas }}}\right)^{2}\right)^{1 / 2} \\
\sigma_{\bar{\rho}, \text { tot }}^{2}=\sigma_{\bar{\rho}, \text { meas }}^{2}+2 \sigma_{d}^{2}
\end{gathered}
$$

where $s$ and $\sigma_{s}$ are the plate scale and associated uncertainty in mas pixel ${ }^{-1}, \bar{\rho}_{\text {meas }}$ and $\sigma_{\bar{\rho}, \text { meas }}$ are the mean and standard deviation of the measured separations for the individual images (in pixels), and $\sigma_{d}$ is the typical residual positional displacement after applying the distortion correction (in pixels, one for each

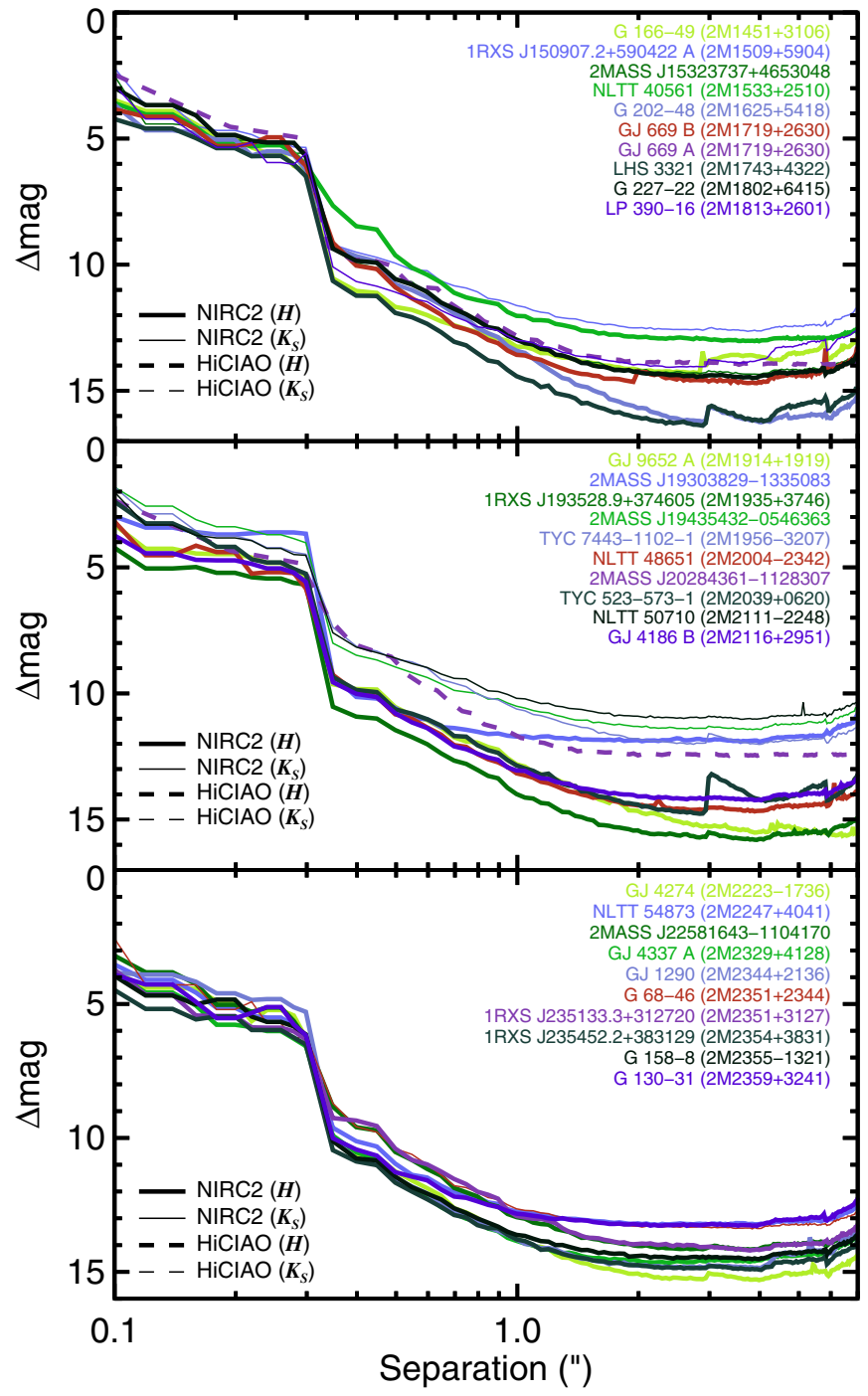

Figure 5. $7 \sigma$ contrast curves from our survey (continued).

binary component). Likewise, the P.A. $(\theta)$ is

$$
\theta=\bar{\theta}_{\text {meas }}+\theta_{\text {North }} \pm\left(\sigma_{\theta, \text { meas }}^{2}+\sigma_{\theta, \text { North }}^{2}\right)^{1 / 2}
$$

where $\bar{\theta}_{\text {meas }}$ is the mean P.A. of the individual images, $\theta_{\text {North }}$ is the orientation of the detector relative to north, $\sigma_{\theta}$, meas is the standard deviation of the P.A. measurements, and $\sigma_{\theta \text {, North }}$ is the uncertainty in the sky orientation on the detector.

\subsubsection{Faint Point Sources}

Astrometry and photometry for faint point sources are measured from our final processed images. The error budget for the separation measurement consists of positional uncertainties of the star behind the mask, centroid errors for the candidate, and systematic errors in the distortion solution (Equation (3)). For NIRC2, we adopt random measurement uncertainties $\left(\sigma_{\rho \text {,meas }}\right)$ of 0.3 pixel, which is typical of our binary star measurements.

The dominant source of astrometric uncertainty for our $\mathrm{HiCIAO}$ data are from the image registration process since the peak of the PSF is hidden under the opaque 300 mas focal plane mask. To estimate the typical magnitude of this uncertainty, we use our ADI sequence of the $\sim 2$ " binary system 

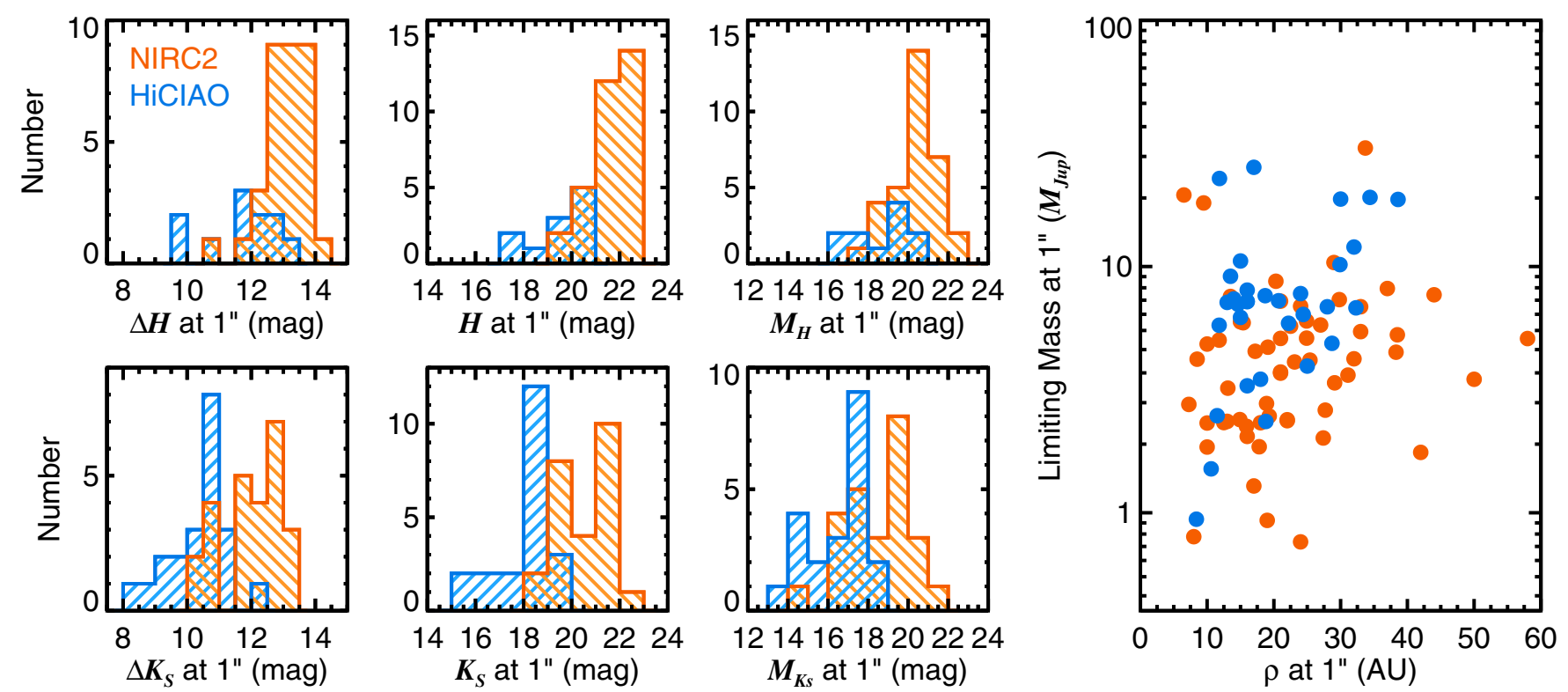

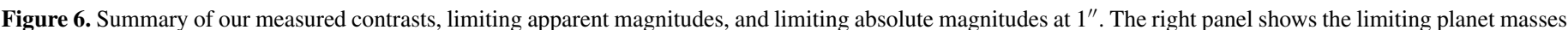

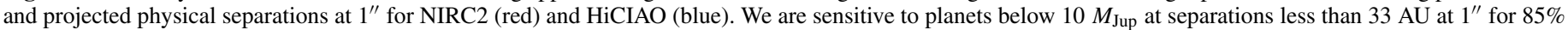
of our sample.

GJ $3030 \mathrm{AB}$. The $R$-band magnitude of this system from UCAC4 ( $\sim 12.2 \mathrm{mag}$ ) is typical for our targets and, as with most of our HiCIAO observations in this program, these data were taken in unusually poor seeing conditions (1.5-2".0). Here GJ 3030 A was behind the mask and the following test was performed on the B component. We first determine the centroid of the companion GJ $3030 \mathrm{~B}$ in each image, then mask the central 150 mas, fit a $2 \mathrm{D}$ elliptical Gaussian to the wing of the PSF following our general method for HiCIAO image registration (see Section 4.1), and compute the relative difference of the inferred to the actual measured stellar position. The inferred position is generally $\sim 1-2.5$ pixel from the actual center (the mean value is 1.6 pixel for this representative sequence), so we conservatively adopt an uncertainty of 2 pixel, or $\sim 20$ mas, for the HiCIAO registration term $\left(\sigma_{\bar{\rho}, \text { meas }}\right)$. For the random error term $\sigma_{\theta \text {,meas }}$ we adopt characteristic uncertainties from our moderate-contrast binary star astrometry of 0.2 and 0.1 for NIRC2 and HiCIAO, respectively.

Position angle uncertainties originate from centroid errors for the candidate companion, systematic errors in the north alignment, and, for HiCIAO, uncertainties associated with image registration. In this latter case we add an additional term, $\sigma_{\text {reg }}$, in quadrature with the random and systematic errors in Equation (5). $\sigma_{\text {reg }}$ refers to the angular uncertainty associated with a positional error (from image registration) orthogonal to the primary-candidate companion direction; for HiCIAO we adopt a 2 pixel positional uncertainty, so $\sigma_{\text {reg }}$ (in deg) scales as $\arcsin \left(2 \operatorname{pixel} / \rho_{\text {meas }}\right)$.

Many of the wide-separation $\left(\gtrsim 5^{\prime \prime}\right)$ candidates identified in first epoch imaging were followed up with the NIRC2 wide camera mode, which has a plate scale of $39.884 \pm 0.039$ mas pixel ${ }^{-1}$ (Pravdo et al. 2006) and FOV $\left(\approx 40^{\prime \prime} \times 40^{\prime \prime}\right)$. For these observations, we use the distortion solution from $\mathrm{Fu}$ et al. (2014) and estimate a residual positional uncertainty $\sigma_{d}$ of 1 pixel from the residual map. ${ }^{10}$ Even behind the partly opaque

\footnotetext{
10 http://www2.keck.hawaii.edu/inst/nirc2/dewarp.html
}

coronagraph, the primary stars were usually saturated in this wide camera mode. Although this had a minimal impact on relative astrometry, it prevented accurate relative photometry for these observations.

Aperture photometry is measured for all point sources to derive contrasts relative to the star. We use aperture radii of 6 pixel for candidates identified in our NIRC2 data. For our HiCIAO observations, which typically suffered from poor seeing and modest AO correction, we use larger aperture radii of 15 pixel. Photometric errors incorporate measurement errors computed at the source location in the noise map, uncertainties in the coronagraph transmission (for NIRC2), uncertainties in the neutral density filters (for HiCIAO, when applicable), and uncertainties in the measured flux of the primary star itself. This last term is the standard deviation of flux measurements from the star (behind the mask for NIRC2, and from unsaturated images for HiCIAO).

\section{SURVEY RESULTS}

Out of 122 targets imaged in our survey, 44 are close stellar binaries, 27 of which are new or spatially resolved for the first time. We discovered four new young brown dwarf companions confirmed to be comoving with their host stars. Over 150 planet candidates were identified out to projected separations of $\approx 400 \mathrm{AU}$; we recovered the majority $(56 \%)$ of these in secondepoch imaging, and all of these are background stars. Below, we describe these results in detail.

\subsection{Substellar Companions from the PALMS Survey}

Our four brown dwarf discoveries span masses of 30-70 $M_{\mathrm{Jup}}$ at projected separations of 6-190 AU. Two have already been published as part of this series (1RXS J235133.3+312720 B in Bowler et al. 2012b; GJ 3629 B in Bowler et al. 2012a) and here we present two new companions, 1RXS J034231.8+121622 B and 2MASS J15594729+4403595 B. Note that the young stars G 196-3 A (2MASS J10042148+5023135) and NLTT 22741 A (2MASS J09510459+3558098; LP 261-75) in our sample have 

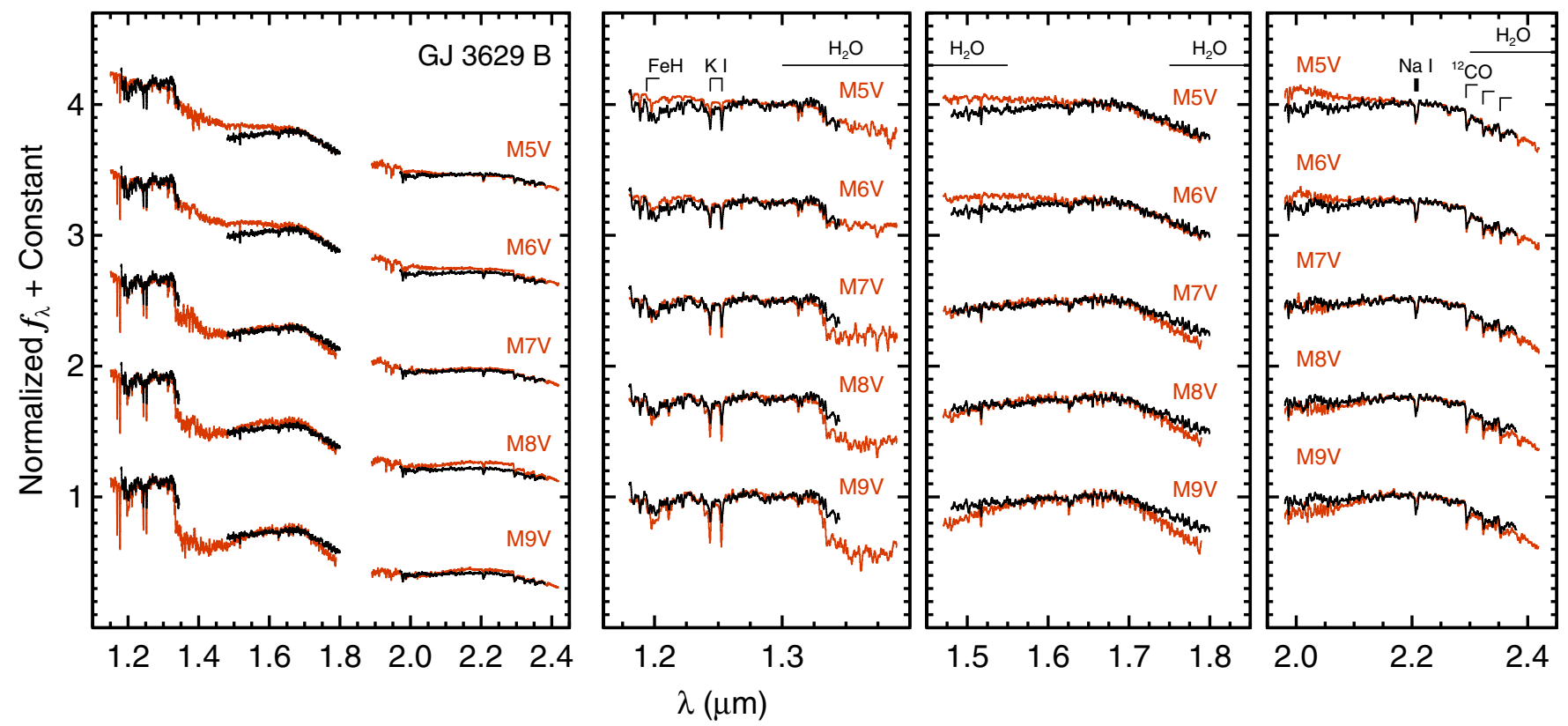

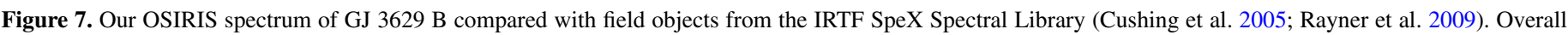

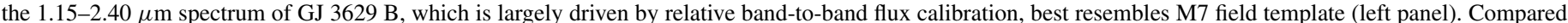

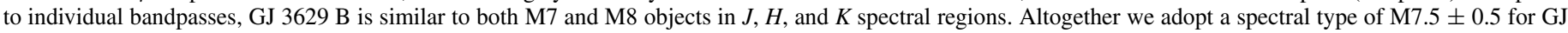

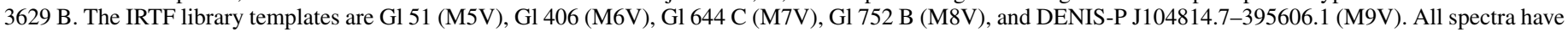
been smoothed to a common resolving power of $R \approx 2000$.

previously known wide-separation brown dwarf companions with L spectral types (Rebolo et al. 1998; Reid \& Walkowicz 2006). However, neither companion falls in the FOV of our observations so they are not included in our statistical analysis (Section 6). Below we summarize our discoveries and present new photometry and astrometry for each system.

\subsubsection{RXS J235133.3+312720 B (2MASS J23513366+3127229)}

1RXS J235133.3+312720 B is a $32 \pm 6 M_{\text {Jup }}$ companion to the active M2.0 star 1RXS J235133.3+312720 A and was the first discovery from our PALMS survey (Bowler et al. 2012b). Shkolnik et al. (2012), Schlieder et al. (2012b), and Malo et al. (2013) independently identify the primary as a likely member of the AB Dor YMG ( $120 \mathrm{Myr})$ based on its kinematics and activity. We found a near-IR spectral type of $\mathrm{LO}_{-1}^{+2}$ for the companion along with subtle spectroscopic hints of low surface gravity (Bowler et al. 2012b). Based on the photometric distance to the primary $(50 \pm 10 \mathrm{pc})$, the 2 .' 4 projected separation corresponds to $120 \pm 20 \mathrm{AU}$.

In Bowler et al. (2012b), we presented two epochs of relative astrometry for 1RXS J235133.3+312720 AB based on AO imaging with NIRC2 in 2011 in the $H$ and $K^{\prime}$ bands. In addition, we also obtained seeing-limited relative photometry from IRTF in $Y J H K s$ filters. Here we present new, more precise 1-3.8 $\mu \mathrm{m}$ relative photometry obtained with NIRC2 in 2013 (Table 7). We infer a $Y-J$ color of $1.17 \pm 0.17 \mathrm{mag}$ for $1 \mathrm{RXS} \mathrm{J} 235133.3+312720 \mathrm{~B}$ assuming a $Y-J$ color of $0.459 \pm 0.001 \mathrm{mag}$ for the primary, which is the mean color for M2.0 dwarfs from Rayner et al. (2009). Based on the MKO photometry of the primary from Bowler et al. (2012b), we derive the following colors for 1RXS J235133.3+312720 B: $(J-H)_{\mathrm{MKO}}=0.73 \pm 0.12 \mathrm{mag},(H-K)_{\mathrm{MKO}}=0.64 \pm$ $0.08 \mathrm{mag},(J-K)_{\mathrm{MKO}}=1.37 \pm 0.12 \mathrm{mag}$. Finally, based on the typical $K_{\mathrm{MKO}}-L^{\prime}$ color of $0.2 \pm 0.1 \mathrm{mag}$ for M2 dwarfs from Golimowski et al. (2004), we derive a $K_{\mathrm{MKO}}-L^{\prime}$ color of $0.8 \pm 0.2$ mag for the companion. Compared to typical colors of M/L dwarfs (e.g., Bowler et al. 2012a; Golimowski et al. 2004), our new photometry for 1 RXS J235133.3+312720 B corresponds to spectral types of $\approx \mathrm{L} 0-\mathrm{L} 3$, which is consistent with our published classification based on near-IR spectroscopy.

Altogether, our astrometry over $3 \mathrm{yr}$ shows tentative signs of orbital motion. The reduced $\chi^{2}$ values for constant and linear fits to the separation measurements are 1.58 and 1.38 , respectively. Reduced $\chi^{2}$ values for the P.A. are 3.91 and 0.47 for the constant and linear models, suggesting a slight change of $-0.076 \pm$ $0.017 \mathrm{yr}^{-1}$. Additional astrometry in the future will be needed for confirmation.

\subsubsection{GJ 3629 B (2MASS J10512059+3607255 B)}

GJ $3629 \mathrm{~B}$ is a modest-contrast companion located a mere 0.2 from its active M3.0Ve host star GJ 3629 A (Bowler et al. 2012a). Based on its photometric distance of $22 \pm 3$ pc and age of 25-300 Myr (Shkolnik et al. 2009), Bowler et al. inferred a mass of $46 \pm 16 M_{\text {Jup }}$ for the companion. Recently, Dittmann et al. (2013) measured a parallactic distance of $32.3 \pm$ $2.4 \mathrm{pc}$ to the system. This corresponds to a somewhat higher luminosity of $\log \left(L / L_{\odot}\right)=-2.89 \pm 0.10$ dex. At the system age of 25-300 Myr, the Burrows et al. (1997) evolutionary models imply a mass much closer to the hydrogen burning limit. Assuming a log-normal luminosity distribution and a linearly uniform age distribution, the resulting mass distribution from Monte Carlo simulations has a median value of $64 M_{\text {Jup }}$. The $68.3 \%$ confidence range about the median is 41-94 $M_{\mathrm{Jup}}$, and the $95.4 \%$ range spans $31-114 M_{\text {Jup }}$. Altogether, the probability that GJ $3629 \mathrm{~B}$ is substellar $\left(<75 M_{\text {Jup }}\right.$ ) is $62 \%$.

Figure 7 shows our resolved 1.15-2.4 $\mu \mathrm{m}$ Keck/OSIRIS spectrum of GJ 3629 B compared to M5-M8 field stars from the IRTF SpeX Spectral Library (Cushing et al. 2005; Rayner 
Table 6

Spectral Types and Gravity Indices for Substellar Companions

\begin{tabular}{|c|c|c|c|c|c|c|c|c|}
\hline Object & NIR SpT & $\begin{array}{l}\mathrm{FeH}_{z} \\
\text { Index }\end{array}$ & $\begin{array}{l}\mathrm{FeH}_{J} \\
\text { Index }\end{array}$ & $\begin{array}{c}\mathrm{VO}_{z} \\
\text { Index }\end{array}$ & $\begin{array}{c}\mathrm{K} \mathrm{I}_{J} \\
\text { Index }\end{array}$ & $\begin{array}{c}H \text {-cont } \\
\text { Index }\end{array}$ & $\begin{array}{l}\text { Gravity } \\
\text { Score }^{\mathrm{a}}\end{array}$ & $\begin{array}{c}\text { Gravity } \\
\text { Class }\end{array}$ \\
\hline GJ $3629 \mathrm{~B}$ & $\mathrm{M} 7.5 \pm 0.5$ & $\ldots$ & $1.098 \pm 0.015$ & $\cdots$ & $1.055 \pm 0.006$ & $1.003 \pm 0.004$ & $\mathrm{nn} 12$ & INT-G? \\
\hline 1RXS J034231.8+121622 B & $\mathrm{L} 0 \pm 1$ & $\ldots$ & $\ldots$ & $\ldots$ & $\ldots$ & $0.948 \pm 0.004$ & nnn1 & INT-G? \\
\hline 2MASS J15594729+4403595 B & $\mathrm{M} 8.0 \pm 0.5$ & $1.097 \pm 0.008$ & $1.087 \pm 0.010$ & $1.049 \pm 0.006$ & $1.058 \pm 0.004$ & $1.018 \pm 0.006$ & $\ln 12$ & INT-G \\
\hline
\end{tabular}

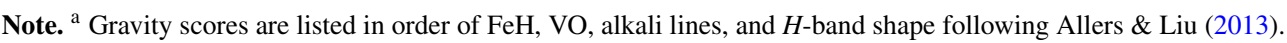

et al. 2009). Overall the spectrum of GJ 3629 B is typical of an ultracool $\mathrm{M}$ dwarf, exhibiting deep $\approx 1.4 \mu \mathrm{m}$ and $1.9 \mu \mathrm{m}$ steam bands, strong $2.2935 \mu \mathrm{m} \mathrm{CO}$ band heads, $\mathrm{FeH}$ absorption at $\approx 1.2 \mu \mathrm{m}$, and $\mathrm{KI}$ and Na I doublets at $1.25 \mu \mathrm{m}$ and $2.21 \mu \mathrm{m}$. GJ $3629 \mathrm{~B}$ is an excellent match to the M7 template across the entire spectrum (left-most panel) and M7-M8 objects among individual bandpasses (right three panels). Altogether we adopt a spectral type of M7.5 \pm 0.5 . Compared to a low- and highgravity templates from Allers \& Liu (2013) in Figure 8, there are no obvious signs of low surface gravity in GJ 3629 B, which is prominently manifested as a angular $H$-band shape and shallow $J$-band alkali line strengths in young brown dwarfs (e.g., McLean et al. 2000; Allers et al. 2007). Our OSIRIS spectrum does not span the entire wavelength range to compute all gravity indices using the Allers \& Liu (2013) scheme, but the $\mathrm{K}_{\mathrm{I}_{J}}$ and $H$-cont indices tentatively show signs of youth (Table 6 and Figure 9).

We also take the opportunity to update the physical properties of the primary with this new trigonometric distance. Following the same methods as in Bowler et al. (2012a), the luminosity of the primary is $\log \left(L_{\mathrm{Bol}} / L_{\odot}\right)=-1.54 \pm 0.08$ dex and its mass is $0.3-0.5 M_{\odot}$ using the Baraffe et al. (1998) evolutionary models. Taking into account the updated component masses and projected separation $(6.5 \pm 0.5 \mathrm{AU})$, the expected orbital period for this system is $30_{-13}^{+30} \mathrm{yr}$ assuming a projected-to-physical conversion scale of $1.16_{-0.31}^{+0.81}$ from Dupuy \& Liu (2011). Our new astrometry taken with HiCIAO only a few months after our last published epoch is consistent with our previously reported measurements. Finally, we note that the system kinematics, $U V W=\{-28.9 \pm 0.8,-15.8 \pm 1.0,-0.9 \pm 0.5\} \mathrm{km} \mathrm{s}^{-1}$, do not correspond to any known moving group.

\subsubsection{RXS J034231.8+121622 B (2MASS J03423180+1216225 B)}

1RXS J034231.8+121622 A is an active M4.0Ve star detected in the ROSAT and GALEX surveys (Riaz et al. 2006). Shkolnik et al. (2009) first noted hints of youth (60-300 Myr) from its X-ray emission and ruled out spectroscopic binarity from two epochs of high-resolution $(R \sim 58,000)$ optical spectroscopy.

Recently, Dittmann et al. (2013) measured a parallactic distance of $23.9 \pm 1.1 \mathrm{pc}$ to this star. Its distance and radial velocity $\left(35.4 \pm 0.4 \mathrm{~km} \mathrm{~s}^{-1}\right)$ from Shkolnik et al. (2012) imply $U V W$ space velocities of $\{-39.0 \pm 0.6,-13.4 \pm 0.8$, $\left.-6.8 \pm 0.7 \mathrm{~km} \mathrm{~s}^{-1}\right\}$, which are similar to, though not formally consistent with, the Hyades moving group $\left(U V W_{\text {Hyades }}=\right.$ $\{-41.7,-19.3,-1.1\} \pm 0.4 \mathrm{~km} \mathrm{~s}^{-1}$; Perryman et al. 1998). 1RXS J034231.8+121622 A also shares a similar sky position with the Hyades and is only 24 pc from the cluster center (Röser et al. 2011), suggesting a possible association with the larger but less coherent Hyades Supercluster or Stream (Eggen 1958). However, the origin and relationship of this kinematic
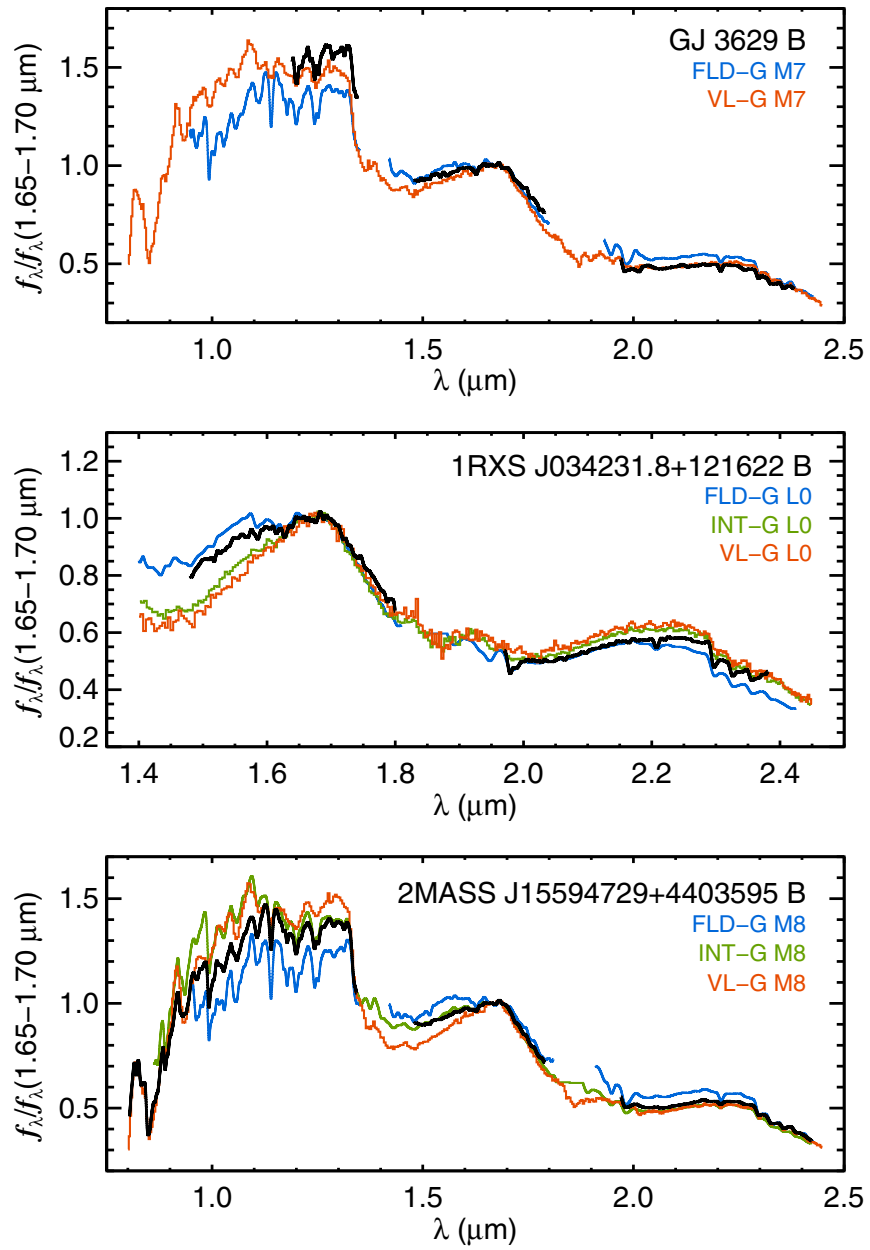

Figure 8. Comparison of GJ 3629 B, 1RXS J034231.8+121622 B, and 2MASS J15594729+4403595 B with very low gravity (VL-G), intermediate-gravity (INT-G), and field high-gravity (FLD-G) ultracool objects from Allers \& Liu (2013). GJ 3629 B does not appear to differ substantially from the FLD-G M7 object. The $H$-band shape of 1RXS J034231.8+121622 B is somewhat less angular than the INT-G L0 template but is noticeably more pronounced than the field object. 2MASS J15594729+4403595 B agrees well with the INT-G M8 template. The comparison objects from Allers \& Liu (2013) are 2MASSJ000342272822410 (FLD-G M7), 2MASS J03350208+2342356 (VL-G M7), 2MASS J17312974+2721233 (FLD-G L0), 2MASS J15525906+2948485 (INT-G L0), 2MASS J22134491-2136079 (VL-G L0), 2MASS J08040580+615333 (FLDG M8), and 2MASS J00192626+4614078 (INT-G M8). TWA 27 A (VL-G M8) is from Looper et al. (2007). All spectra are smoothed to $R \sim 120$ and normalized to the $1.65-1.70 \mu \mathrm{m}$ region.

overdensity with the Hyades cluster is not clear and is unlikely to be useful for age-dating purposes (e.g., Famaey et al. 2005). So for this work we adopt the 60-300 Myr statistical age constraint from Shkolnik et al. (2009) based on the X-ray luminosity and spectroscopic youth indicators of 1RXS J034231.8+121622 A. 

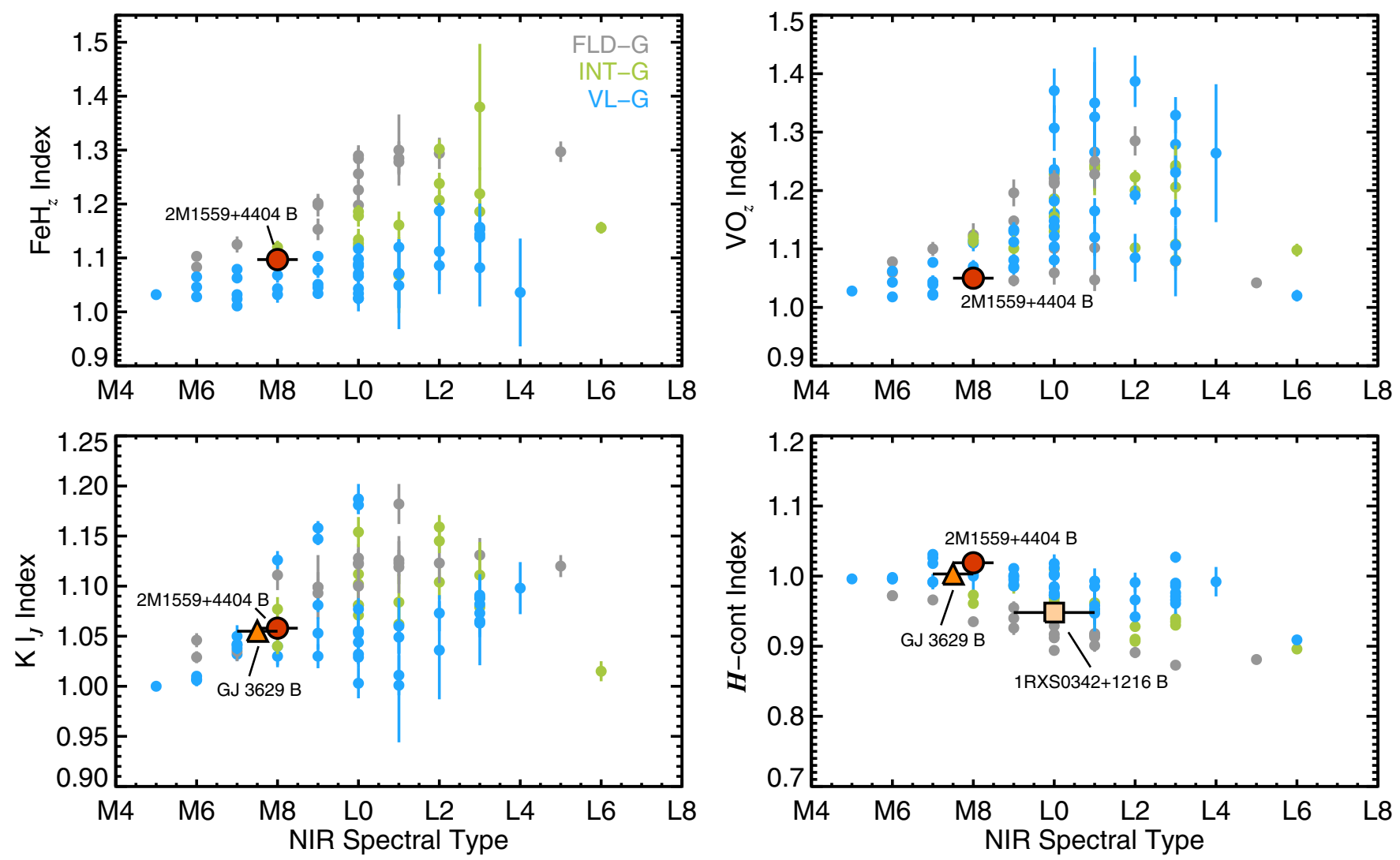

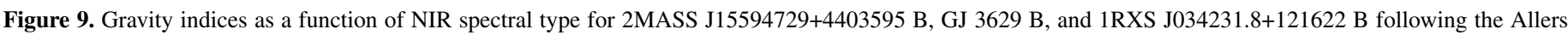

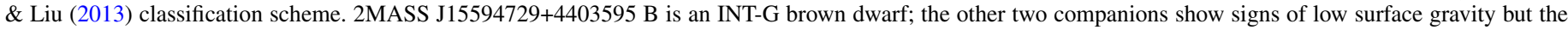

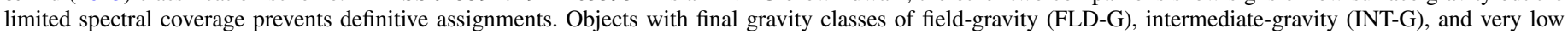
gravity (VL-G) from Allers \& Liu (2013) are plotted in gray, green, and blue for comparison.

Janson et al. (2012) used Lucky imaging to resolve a close ( 0.8 ) candidate companion, 1 RXS J034231.8+121622 B, at two epochs in 2008. However, they were not able to distinguish a background object from a comoving companion from these data. Their contrast measurement in $z$ band (5.20 $\pm 0.27 \mathrm{mag})$ imply a spectral type of $\gtrsim \mathrm{L} 0$ for the companion.

We imaged 1RXS J034231.8+121622 on 2012 August 23 UT and 2013 January 17 UT with NIRC2 in the $Y, J, H, K_{S}$, and $L^{\prime}$ bands (Table 7 ). The companion was easily identified in all the data with contrasts between 4.3-3.6 mag (Figure 10). Figure 11 shows our astrometry and that from Janson et al. between 2008 and 2013 compared to the expected track from a background object. We confirm that 1RXS J034231.8+121622 $\mathrm{B}$ is physically bound and detect slight orbital motion in both P.A. and separation. The reduced $\chi^{2}$ value for a constant fit in separation is 6.97 and for a linear fit is 0.98. Similarly, for the P.A., the constant fit gives 6.75 and 5.23. Removing the 2012.645 epoch $Y$-band P.A. point, in which the companion was only identified in three exposures, gives a reduced $\chi^{2}$ value of 3.67 and 0.46 for the constant and linear P.A. fits, respectively. These imply orbital motion of $-8.1 \pm 1.5$ mas $\mathrm{yr}^{-1}$ in separation and $+0.33 \pm 0.09 \mathrm{yr}^{-1}$ in P.A. At a distance of $23.9 \pm 1.1 \mathrm{pc}$, the projected separation of the pair is $19.8 \pm 0.9 \mathrm{AU}$.

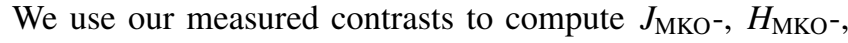
and $K_{S}$-band magnitudes for 1RXS J034231.8+121622 B based on photometry of the primary, which was first converted from the Two Micron Sky Survey (2MASS) to MKO filter system for the $J$ and $H$ filters with the relations from Leggett et al. (2006).
Based on the typical $Y-J$ color of $0.524 \pm 0.01$ mag for M4 stars from Rayner et al. (2009), we derive a $Y-J$ color of $0.86 \pm$ $0.13 \mathrm{mag}$ for 1RXS J034231.8+121622 B, which suggests a photometric spectral type of M8 \pm 1 .

Figure 12 shows our resolved Keck/OSIRIS $H$ - and $K$ band spectra of 1RXS J034231.8+121622 B compared to field templates. 1RXS J034231.8+121622 B is most similar to L0-L1 objects in the 1.4-2.4 $\mu \mathrm{m}$ region and in $H$-band alone. The $K$ band spectrum resembles field M9-L0 templates. Altogether we adopt a spectral type of $\mathrm{LO} \pm 1$. Compared to younger L0 objects from Allers \& Liu (2013) in Figure 8, the blue side of the $H$ band of 1RXS J034231.8+121622 B appears somewhat shallower than the $H$-band spectral shape of the field object, but not as much as the intermediate- or very low gravity brown dwarfs (Figure 9).

Using this spectral type and the system distance, we compute an $H$-band bolometric correction from Liu et al. (2010b) and a bolometric luminosity of $\log L / L_{\odot}=-3.81 \pm 0.05 \mathrm{dex}$. Uncertainties in distance, spectral type, and photometry are incorporated into our final error in an Monte Carlo fashion. Based on the age of the system, the evolutionary models of Burrows et al. (1997) imply a mass of $35 \pm 8 M_{\text {Jup }}$.

\subsubsection{MASS J15594729+4403595 B}

2MASS J15594729+4403595 A is an M2.0 star exhibiting $\mathrm{H} \alpha$ emission and saturated X-ray emission (Riaz et al. 2006). As part of an ongoing search for young low-mass members, E. L. Shkolnik et al. (in preparation) identify this active star from its 
Table 7

Astrometry of Stellar and Substellar Companions

\begin{tabular}{|c|c|c|c|c|c|c|c|c|c|c|c|}
\hline Name & 2MASS ID & $\begin{array}{l}\text { Epoch } \\
\text { (UT) }\end{array}$ & Instrument & Filt. & $N_{\text {images }}$ & $\begin{array}{l}\text { Separation } \\
\text { (mas) }\end{array}$ & $\begin{array}{l}\text { P.A. } \\
\left({ }^{\circ}\right)\end{array}$ & $\Delta$ mag & $\begin{array}{l}m_{\mathrm{A}}{ }^{\mathrm{a}} \\
(\mathrm{mag})\end{array}$ & $\begin{array}{c}m_{\mathrm{B}}^{\mathrm{b}} \\
(\mathrm{mag})\end{array}$ & $\begin{array}{c}\text { FWHM } \\
\text { (mas) }\end{array}$ \\
\hline \multicolumn{12}{|c|}{ Substellar Companions } \\
\hline \multirow[t]{5}{*}{ 1RXS J034231.8+121622 AB } & J03423180+1216225 & 2012.645 & $\mathrm{NIRC} 2 / \mathrm{N}$ & $Y$ & 3 & $850 \pm 30$ & $17.8 \pm 0.2$ & $4.25 \pm 0.12$ & $10.70 \pm 0.02^{\mathrm{c}}$ & $14.95 \pm 0.12^{\mathrm{c}}$ & $68 \pm 16$ \\
\hline & & 2012.645 & $\mathrm{NIRC} 2 / \mathrm{N}$ & $J$ & 10 & $829 \pm 2$ & $18.5 \pm 0.2$ & $3.91 \pm 0.05$ & $10.19 \pm 0.02$ & $14.10 \pm 0.05$ & $52 \pm 4$ \\
\hline & & 2012.645 & $\mathrm{NIRC} 2 / \mathrm{N}$ & $H$ & 10 & $831 \pm 2$ & $18.71 \pm 0.07$ & $3.93 \pm 0.05$ & $9.58 \pm 0.019$ & $13.51 \pm 0.05$ & $53 \pm 4$ \\
\hline & & 2012.645 & $\mathrm{NIRC} 2 / \mathrm{N}$ & $K_{S}$ & 10 & $832.4 \pm 1.0$ & $18.70 \pm 0.04$ & $3.76 \pm 0.07$ & $9.31 \pm 0.018$ & $13.07 \pm 0.07$ & $54.8 \pm 1.8$ \\
\hline & & 2013.044 & $\mathrm{NIRC} 2 / \mathrm{N}$ & $L^{\prime}$ & 10 & $822 \pm 8$ & $19.1 \pm 0.7$ & $3.56 \pm 0.17$ & $9.22 \pm 0.10^{\mathrm{d}}$ & $12.78 \pm 0.19^{\mathrm{d}}$ & $110 \pm 9$ \\
\hline GJ 3629 AB & $\mathrm{J} 10512059+3607255$ & 2012.357 & HiCIAO & $H$ & 28 & $206 \pm 19$ & $119.6 \pm 2.3$ & $3.3 \pm 0.3$ & $8.87 \pm 0.02$ & $12.2 \pm 0.3$ & $79 \pm 11$ \\
\hline \multirow[t]{4}{*}{ 2MASS J15594729+4403595 AB } & $\mathrm{J} 15594729+4403595$ & 2012.357 & HiCIAO & $Y$ & 10 & $5646 \pm 27$ & $284.47 \pm 0.10$ & $5.06 \pm 0.04$ & $8.98 \pm 0.02^{\mathrm{c}}$ & $14.04 \pm 0.05^{\mathrm{c}}$ & $94 \pm 6$ \\
\hline & & 2012.357 & HiCIAO & $J$ & 10 & $5647 \pm 27$ & $284.48 \pm 0.10$ & $4.72 \pm 0.03$ & $8.52 \pm 0.02$ & $13.24 \pm 0.04$ & $82 \pm 8$ \\
\hline & & 2012.357 & HiCIAO & $H$ & 20 & $5647 \pm 15$ & $284.46 \pm 0.10$ & $4.76 \pm 0.03$ & $7.86 \pm 0.02$ & $12.62 \pm 0.04$ & $73 \pm 4$ \\
\hline & & 2012.357 & HiCIAO & $K_{S}$ & 20 & $5644 \pm 22$ & $284.47 \pm 0.10$ & $4.11 \pm 0.02$ & $7.65 \pm 0.02$ & $11.76 \pm 0.03$ & $78 \pm 3$ \\
\hline \multirow[t]{6}{*}{ 1RXS J235133.3+312720 AB } & $\mathrm{J} 23513366+3127229$ & 2013.626 & $\mathrm{NIRC} 2 / \mathrm{N}$ & $Y$ & 10 & $2391 \pm 4$ & $91.63 \pm 0.02$ & $6.24 \pm 0.14$ & $10.28 \pm 0.02^{\mathrm{c}}$ & $16.52 \pm 0.14^{\mathrm{c}}$ & $31.4 \pm 1.8$ \\
\hline & & 2013.626 & $\mathrm{NIRC} 2 / \mathrm{N}$ & $J$ & 14 & $2391 \pm 3$ & $91.647 \pm 0.015$ & $5.53 \pm 0.10$ & $9.83 \pm 0.02$ & $15.36 \pm 0.10$ & $33.6 \pm 1.6$ \\
\hline & & 2013.626 & $\mathrm{NIRC} 2 / \mathrm{N}$ & $H$ & 8 & $2390.7 \pm 1.1$ & $91.65 \pm 0.010$ & $5.39 \pm 0.05$ & $9.18 \pm 0.019$ & $14.57 \pm 0.05$ & $40.7 \pm 0.8$ \\
\hline & & 2013.626 & $\mathrm{NIRC} 2 / \mathrm{N}$ & $K s$ & 5 & $2391.2 \pm 1.1$ & $91.63 \pm 0.03$ & $4.96 \pm 0.05$ & $8.98 \pm 0.018$ & $13.94 \pm 0.05$ & $49.4 \pm 0.3$ \\
\hline & & 2013.626 & $\mathrm{NIRC} 2 / \mathrm{N}$ & $K$ & 6 & $2391.6 \pm 1.7$ & $91.643 \pm 0.014$ & $4.98 \pm 0.05$ & $8.95 \pm 0.02^{\mathrm{e}}$ & $13.93 \pm 0.05^{\mathrm{e}}$ & $50.4 \pm 0.3$ \\
\hline & & 2013.626 & $\mathrm{NIRC} 2 / \mathrm{N}$ & $L^{\prime}$ & 20 & $2390 \pm 5$ & $91.64 \pm 0.08$ & $4.40 \pm 0.19$ & $8.99 \pm 0.10^{\mathrm{d}}$ & $13.39 \pm 0.21^{\mathrm{d}}$ & $83.6 \pm 0.4$ \\
\hline \multicolumn{12}{|c|}{ Stellar Companions } \\
\hline G $217-32 \mathrm{AB}$ & $\mathrm{J} 00074264+6022543$ & 2010.628 & $\mathrm{NIRC} 2 / \mathrm{N}$ & $H$ & 3 & $528.9 \pm 0.9$ & $74.22 \pm 0.11$ & $0.475 \pm 0.011$ & $8.87 \pm 0.02$ & $9.35 \pm 0.03$ & $44.3 \pm 0.3$ \\
\hline \multirow[t]{3}{*}{ 1RXS J001557.5-163659 AB } & J00155808-1636578 & 2011.634 & $\mathrm{NIRC} 2 / \mathrm{N}$ & $H$ & 10 & $104.5 \pm 0.9$ & $90.37 \pm 0.05$ & $0.06 \pm 0.05$ & $8.91 \pm 0.03$ & $8.97 \pm 0.03$ & $45 \pm 2$ \\
\hline & & 2012.644 & $\mathrm{NIRC} 2 / \mathrm{N}$ & $K_{S}$ & 20 & $<59$ & $\ldots$ & $\ldots$ & $\ldots$ & $\ldots$ & $58.9 \pm 1.0$ \\
\hline & & 2012.770 & $\mathrm{NIRC} 2 / \mathrm{N}$ & $H$ & 10 & $<54$ & $\ldots$ & $\ldots$ & $\ldots$ & $\ldots$ & $54.1 \pm 1.3$ \\
\hline GJ $3030 \mathrm{AB}$ & J00215781+4912379 & 2011.986 & HiCIAO & $H$ & 13 & $2267 \pm 14$ & $300.30 \pm 0.11$ & $2.16 \pm 0.04$ & $8.59 \pm 0.02$ & $10.75 \pm 0.04$ & $120 \pm 20$ \\
\hline \multirow[t]{2}{*}{ G 132-50 Aab } & J01034013+4051288 & 2011.989 & HiCIAO & $H$ & 5 & $270 \pm 15$ & $308 \pm 3$ & $2.8 \pm 0.3$ & $7.55 \pm 0.07$ & $10.3 \pm 0.3$ & $100 \pm 30$ \\
\hline & & 2011.989 & HiCIAO & $K_{S}$ & 14 & $264 \pm 17$ & $308.9 \pm 1.2$ & $2.34 \pm 0.18$ & $7.45 \pm 0.03$ & $9.78 \pm 0.16$ & $87 \pm 6$ \\
\hline \multirow[t]{2}{*}{ NLTT 6549 AB } & J01581361+4844197 & 2011.069 & HiCIAO & $H$ & 15 & $<120$ & & & & & $120 \pm 30$ \\
\hline & & 2011.634 & $\mathrm{NIRC} 2 / \mathrm{N}$ & $H$ & 5 & $49.6 \pm 1.2$ & $51 \pm 2$ & $1.44 \pm 0.09$ & $8.69 \pm 0.03$ & $10.13 \pm 0.07$ & $40.7 \pm 0.4$ \\
\hline 2MASS J03033668-2535329 AB & J03033668-2535329 & 2011.989 & HiCIAO & $K_{S}$ & 25 & $830 \pm 14$ & $359.84 \pm 0.13$ & $2.99 \pm 0.06$ & $7.17 \pm 0.03$ & $10.16 \pm 0.06$ & $93 \pm 14$ \\
\hline \multirow[t]{5}{*}{ G $160-54$ AB } & J04134585-0509049 & 2012.006 & HiCIAO & $Y$ & 5 & $195 \pm 14$ & $127.10 \pm 0.12$ & $0.177 \pm 0.011$ & $10.91 \pm 0.03^{c}$ & $11.09 \pm 0.03^{\mathrm{c}}$ & $123 \pm 2$ \\
\hline & & 2012.006 & HiCIAO & $J$ & 20 & $195 \pm 14$ & $127.49 \pm 0.08$ & $0.181 \pm 0.012$ & $10.38 \pm 0.03$ & $10.56 \pm 0.03$ & $123 \pm 5$ \\
\hline & & 2012.006 & HiCIAO & $H$ & 20 & $193 \pm 14$ & $128.02 \pm 0.17$ & $0.134 \pm 0.015$ & $9.80 \pm 0.03$ & $9.94 \pm 0.03$ & $111 \pm 7$ \\
\hline & & 2012.006 & HiCIAO & $K_{S}$ & 20 & $195 \pm 14$ & $127.78 \pm 0.14$ & $0.12 \pm 0.03$ & $9.52 \pm 0.03$ & $9.64 \pm 0.03$ & $96 \pm 3$ \\
\hline & & 2012.645 & $\mathrm{NIRC} 2 / \mathrm{N}$ & $K_{S}$ & 10 & $166.7 \pm 1.9$ & $123.2 \pm 0.3$ & $0.42 \pm 0.11$ & $9.39 \pm 0.05$ & $9.81 \pm 0.07$ & $62 \pm 6$ \\
\hline \multirow[t]{5}{*}{ G 160-54 AC } & J04134585-0509049 & 2012.006 & HiCIAO & $Y$ & 5 & $3355 \pm 20$ & $108.50 \pm 0.10$ & $3.039 \pm 0.012$ & $10.91 \pm 0.03^{c}$ & $14.01 \pm 0.03^{\mathrm{c}}$ & $123 \pm 2$ \\
\hline & & 2012.006 & HiCIAO & $J$ & 20 & $3350 \pm 19$ & $108.59 \pm 0.10$ & $2.780 \pm 0.013$ & $10.38 \pm 0.03$ & $13.24 \pm 0.03$ & $123 \pm 5$ \\
\hline & & 2012.006 & HiCIAO & $H$ & 20 & $3349 \pm 14$ & $108.65 \pm 0.10$ & $2.735 \pm 0.014$ & $9.80 \pm 0.02$ & $12.62 \pm 0.03$ & $111 \pm 7$ \\
\hline & & 2012.006 & HiCIAO & $K_{S}$ & 20 & $3348 \pm 17$ & $108.61 \pm 0.10$ & $2.279 \pm 0.013$ & $9.52 \pm 0.02$ & $11.93 \pm 0.03$ & $96 \pm 3$ \\
\hline & & 2012.645 & $\mathrm{NIRC} 2 / \mathrm{N}$ & $K_{S}$ & 10 & $3332 \pm 1.8$ & $108.47 \pm 0.03$ & $2.77 \pm 0.06$ & $9.39 \pm 0.02$ & $12.24 \pm 0.06$ & $62 \pm 6$ \\
\hline 2MASS J04220833-2849053 AB & J04220833-2849053 & 2012.006 & HiCIAO & $H$ & 10 & $745 \pm 14$ & $241.83 \pm 0.11$ & $0.048 \pm 0.011$ & $8.29 \pm 0.02$ & $8.34 \pm 0.02$ & $57 \pm 4$ \\
\hline 2MASS J04472312-2750358 & J04472312-2750358 & 2011.069 & HiCIAO & $K_{S}$ & 5 & $8848 \pm 31$ & $133.80 \pm 0.10$ & $0.57 \pm 0.014$ & $7.33 \pm 0.02$ & $7.90 \pm 0.02$ & $80 \pm 6$ \\
\hline \multirow[t]{2}{*}{ G $81-34 \mathrm{AB}$} & J04492947+4828459 & 2011.072 & HiCIAO & $K_{S}$ & 5 & $637 \pm 14$ & $234.18 \pm 0.13$ & $0.564 \pm 0.016$ & $8.68 \pm 0.03$ & $9.24 \pm 0.03$ & $76 \pm 3$ \\
\hline & & 2011.634 & $\mathrm{NIRC} 2 / \mathrm{N}$ & $H$ & 6 & $636.5 \pm 1.9$ & $237.68 \pm 0.08$ & $0.57 \pm 0.03$ & $8.94 \pm 0.06$ & $9.51 \pm 0.06$ & $40 \pm 2$ \\
\hline 2MASS J06131330-2742054 AB & J06131330-2742054 & 2011.987 & HiCIAO & $K_{S}$ & 17 & $130 \pm 14$ & $215.3 \pm 0.8$ & $0.31 \pm 0.10$ & $7.75 \pm 0.05$ & $8.06 \pm 0.06$ & $70 \pm 20$ \\
\hline LHS 1864 AB & J06434969+5108209 & 2011.987 & HiCIAO & $K_{S}$ & 10 & $1765 \pm 15$ & $271.0 \pm 0.10$ & $0.674 \pm 0.012$ & $8.012 \pm 0.018$ & $8.69 \pm 0.02$ & $62 \pm 3$ \\
\hline 1RXS J091744.5+461229 AB & J09174473+4612246 & 2011.073 & HiCIAO & $K_{S}$ & 10 & $204 \pm 14$ & $37.5 \pm 0.3$ & $0.102 \pm 0.019$ & $7.948 \pm 0.019$ & $8.05 \pm 0.02$ & $55 \pm 7$ \\
\hline
\end{tabular}


Table 7

(Continued)

\begin{tabular}{|c|c|c|c|c|c|c|c|c|c|c|c|}
\hline Name & 2MASS ID & $\begin{array}{l}\text { Epoch } \\
\text { (UT) }\end{array}$ & Instrument & Filt. & $N_{\text {images }}$ & $\begin{array}{l}\text { Separation } \\
\text { (mas) }\end{array}$ & $\begin{array}{c}\text { P.A. } \\
\left({ }^{\circ}\right)\end{array}$ & $\Delta$ mag & $\begin{array}{c}m_{\mathrm{A}}{ }^{\mathrm{a}} \\
(\mathrm{mag})\end{array}$ & $\begin{array}{l}m_{\mathrm{B}}^{\mathrm{b}} \\
(\mathrm{mag})\end{array}$ & $\begin{array}{l}\text { FWHM } \\
\text { (mas) }\end{array}$ \\
\hline \multirow[t]{3}{*}{ GJ $2079 \mathrm{AB}$} & J10141918+2104297 & 2011.228 & $\mathrm{NIRC} 2 / \mathrm{N}$ & $K_{S}$ & 10 & $99 \pm 2$ & $129.99 \pm 0.17$ & $1.87 \pm 0.06$ & $6.44 \pm 0.03$ & $8.31 \pm 0.06$ & $48.2 \pm 0.2$ \\
\hline & & 2011.990 & HiCIAO & $K_{S}$ & 23 & $<83$ & & & & & $83 \pm 9$ \\
\hline & & 2013.094 & $\mathrm{NIRC} 2 / \mathrm{N}$ & $K_{S}$ & 20 & $95 \pm 3$ & $320.2 \pm 1.2$ & $1.8 \pm 0.2$ & $6.45 \pm 0.04$ & $8.25 \pm 0.17$ & $51.4 \pm 1.2$ \\
\hline PYC J10571+0544 AB & $\mathrm{J} 10571139+0544547$ & 2012.390 & $\mathrm{NIRC} 2 / \mathrm{N}$ & $H$ & 14 & $1005.6 \pm 1.3$ & $155.96 \pm 0.04$ & $0.82 \pm 0.02$ & $9.63 \pm 0.02$ & $10.45 \pm 0.03$ & $46 \pm 2$ \\
\hline \multirow[t]{2}{*}{ 2MASS J12062214-1314559 AB } & J12062214-1314559 & 2012.007 & HiCIAO & $H$ & 25 & $422 \pm 15$ & $54.6 \pm 0.3$ & $1.94 \pm 0.02$ & $8.24 \pm 0.04$ & $10.18 \pm 0.04$ & $100 \pm 20$ \\
\hline & & 2012.007 & HiCIAO & $K_{S}$ & 10 & $423 \pm 14$ & $54.62 \pm 0.19$ & $1.90 \pm 0.02$ & $7.99 \pm 0.03$ & $9.89 \pm 0.03$ & $68 \pm 3$ \\
\hline \multirow[t]{3}{*}{ G $13-33 \mathrm{AB}$} & J12225061-0404462 & 2011.990 & HiCIAO & $K_{S}$ & 19 & $<170$ & & & & & $170 \pm 16$ \\
\hline & & 2012.387 & $\mathrm{NIRC} 2 / \mathrm{N}$ & $K_{S}$ & 21 & $57.1 \pm 1.3$ & $64.7 \pm 1.1$ & $0.27 \pm 0.09$ & $9.45 \pm 0.05$ & $9.72 \pm 0.06$ & $71 \pm 6$ \\
\hline & & 2013.095 & $\mathrm{NIRC} 2 / \mathrm{N}$ & $K_{S}$ & 17 & $129.7 \pm 0.9$ & $36.6 \pm 0.09$ & $0.18 \pm 0.05$ & $9.49 \pm 0.03$ & $9.67 \pm 0.03$ & $79 \pm 16$ \\
\hline LP 735-48 AB & J12264413-1229175 & 2011.987 & HiCIAO & $K_{S}$ & 10 & $1889 \pm 15$ & $71.23 \pm 0.11$ & $0.285 \pm 0.018$ & $8.49 \pm 0.02$ & $8.78 \pm 0.02$ & $119 \pm 18$ \\
\hline \multirow[t]{4}{*}{ GJ $3729 \mathrm{AB}$} & J12290290+4143497 & 2011.070 & HiCIAO & $K_{S}$ & 10 & $<88$ & & & $\ldots$ & & $88 \pm 13$ \\
\hline & & 2011.469 & $\mathrm{NIRC} 2 / \mathrm{N}$ & $H$ & 15 & $50.3 \pm 0.9$ & $255.5 \pm 0.8$ & $0.647 \pm 0.06$ & $8.66 \pm 0.03$ & $9.31 \pm 0.04$ & $44.3 \pm 1.5$ \\
\hline & & 2012.007 & HiCIAO & $H$ & 10 & $<86$ & $\ldots$ & $\ldots$ & $\ldots$ & $\ldots$ & $86 \pm 10$ \\
\hline & & 2013.095 & $\mathrm{NIRC} 2 / \mathrm{N}$ & $K_{S}$ & 20 & $<58$ & $\ldots$ & $\ldots$ & $\ldots$ & $\ldots$ & $58.5 \pm 1.1$ \\
\hline \multirow{2}{*}{ 2MASS J12383713-2703348 AB } & J12383713-2703348 & 2012.007 & HiCIAO & $H$ & 10 & $215 \pm 14$ & $286.4 \pm 0.3$ & $2.25 \pm 0.08$ & $8.21 \pm 0.04$ & $10.46 \pm 0.08$ & $67 \pm 4$ \\
\hline & & 2012.007 & HiCIAO & $K_{S}$ & 10 & $220 \pm 14$ & $286.5 \pm 0.5$ & $2.28 \pm 0.15$ & $7.97 \pm 0.03$ & $10.25 \pm 0.14$ & $74 \pm 5$ \\
\hline GJ $490 \mathrm{Bab}$ & $\mathrm{J} 12574030+3513306$ & 2011.229 & $\mathrm{NIRC} 2 / \mathrm{N}$ & $K_{S}$ & 10 & $171.0 \pm 0.9$ & $310.06 \pm 0.02$ & $0.061 \pm 0.011$ & $8.74 \pm 0.02$ & $8.80 \pm 0.02$ & $49.7 \pm 1.0$ \\
\hline \multirow[t]{2}{*}{ GJ $490 \mathrm{Aab}$} & J12573935+3513194 & 2011.229 & $\mathrm{NIRC} 2 / \mathrm{N}$ & $K_{\text {cont }}$ & 10 & $106.4 \pm 0.9$ & $309.54 \pm 0.13$ & $1.91 \pm 0.05$ & $8.17 \pm 0.03$ & $10.08 \pm 0.05$ & $49.92 \pm 0.11$ \\
\hline & & 2012.037 & $\mathrm{NIRC} 2 / \mathrm{N}$ & $K$ & 9 & $112.8 \pm 1.6$ & $239.8 \pm 0.2$ & $2.06 \pm 0.04$ & $8.14 \pm 0.03^{\mathrm{e}}$ & $10.20 \pm 0.04^{\mathrm{e}}$ & $54 \pm 4$ \\
\hline NLTT 34410 AB & J13323908+3059065 & 2011.387 & $\mathrm{NIRC} 2 / \mathrm{N}$ & $K_{S}$ & 14 & $136.5 \pm 1.1$ & $213.8 \pm 0.5$ & $0.11 \pm 0.06$ & $9.46 \pm 0.04$ & $9.57 \pm 0.04$ & $71 \pm 2$ \\
\hline 2MASS J14215503-3125537 AB & $\mathrm{J} 14215503-3125537$ & 2011.229 & $\mathrm{NIRC} 2 / \mathrm{N}$ & $K_{S}$ & 20 & $70.4 \pm 0.9$ & $59.5 \pm 0.2$ & $0.10 \pm 0.05$ & $9.44 \pm 0.03$ & $9.54 \pm 0.03$ & $66 \pm 3$ \\
\hline LHS $3122 \mathrm{AB}$ & $\mathrm{J} 15493833+3448555$ & 2011.469 & $\mathrm{NIRC} 2 / \mathrm{N}$ & $H$ & 5 & $209.1 \pm 0.9$ & $85.28 \pm 0.04$ & $1.462 \pm 0.012$ & $8.40 \pm 0.02$ & $9.86 \pm 0.03$ & $38.8 \pm 0.3$ \\
\hline 2MASS J16074132-1103073 AB & $\mathrm{J} 16074132-1103073$ & 2012.387 & $\mathrm{NIRC} 2 / \mathrm{N}$ & $K_{S}$ & 6 & $739.4 \pm 1.2$ & $149.64 \pm 0.07$ & $0.014 \pm 0.014$ & $9.74 \pm 0.02$ & $9.75 \pm 0.02$ & $75 \pm 15$ \\
\hline GJ $3966 \mathrm{AB}$ & $\mathrm{J} 16352740+3500577$ & 2011.469 & $\mathrm{NIRC} 2 / \mathrm{N}$ & $H$ & 8 & $92.2 \pm 0.9$ & $25.62 \pm 0.12$ & $0.406 \pm 0.06$ & $8.62 \pm 0.03$ & $9.02 \pm 0.04$ & $48 \pm 2$ \\
\hline GJ $3997 \mathrm{AB}$ & $\mathrm{J} 17155010+1900000$ & 2012.390 & $\mathrm{NIRC} 2 / \mathrm{N}$ & $K_{S}$ & 10 & $1672.4 \pm 1.0$ & $271.39 \pm 0.02$ & $1.886 \pm 0.013$ & $6.626 \pm 0.017$ & $8.51 \pm 0.02$ & $56 \pm 3$ \\
\hline LP $447-38 \mathrm{AB}$ & $\mathrm{J} 17182284+1808568$ & 2012.358 & HiCIAO & $H$ & 10 & $411 \pm 14$ & $298.41 \pm 0.12$ & $1.182 \pm 0.012$ & $8.810 \pm 0.017$ & $9.99 \pm 0.02$ & $106 \pm 6$ \\
\hline 2MASS J19560294-3207186 AB & J19560294-3207186 & 2012.358 & HiCIAO & $H$ & 10 & $199 \pm 14$ & $228.4 \pm 0.2$ & $1.22 \pm 0.04$ & $8.65 \pm 0.04$ & $9.87 \pm 0.05$ & $75 \pm 8$ \\
\hline 2MASS J20003177+5921289 AB & $\mathrm{J} 20003177+5921289$ & 2010.628 & $\mathrm{NIRC} 2 / \mathrm{N}$ & $H$ & 1 & 320.4 & 274.9 & 0.029 & 9.81 & 9.84 & 49 \\
\hline 2MASS J20100002-2801410 AB & J20100002-2801410 & 2012.358 & HiCIAO & $H$ & 10 & $676 \pm 14$ & $282.6 \pm 0.12$ & $0.25 \pm 0.02$ & $8.65 \pm 0.05$ & $8.90 \pm 0.05$ & $86 \pm 13$ \\
\hline NLTT $50066 \mathrm{AB}$ & $\mathrm{J} 20531465-0221218$ & 2010.628 & $\mathrm{NIRC} 2 / \mathrm{N}$ & $H$ & 2 & $138.0 \pm 0.9$ & $327.323 \pm 0.014$ & $0.11 \pm 0.05$ & $9.41 \pm 0.03$ & $9.52 \pm 0.04$ & $40.92 \pm 0.10$ \\
\hline \multirow[t]{2}{*}{ GJ $4185 \mathrm{Aab}$} & $\mathrm{J} 21160576+2951511$ & 2011.470 & $\mathrm{NIRC} 2 / \mathrm{N}$ & $H$ & 5 & $54.3 \pm 0.9$ & $354.6 \pm 0.4$ & $0.37 \pm 0.07$ & $8.44 \pm 0.03$ & $8.81 \pm 0.04$ & $42.6 \pm 0.5$ \\
\hline & & 2011.633 & $\mathrm{NIRC} 2 / \mathrm{N}$ & $H$ & 9 & $49.5 \pm 0.9$ & $47.8 \pm 0.8$ & $0.34 \pm 0.09$ & $8.46 \pm 0.04$ & $8.80 \pm 0.05$ & $41.0 \pm 1.3$ \\
\hline PYC J21376+0137 AB & J21374019+0137137 & 2012.388 & $\mathrm{NIRC} 2 / \mathrm{N}$ & $K_{S}$ & 18 & $439.5 \pm 1.4$ & $342.6 \pm 0.14$ & $0.83 \pm 0.03$ & $8.30 \pm 0.02$ & $9.13 \pm 0.03$ & $51.0 \pm 1.6$ \\
\hline 1RXS J221419.3+253411 AB & $\mathrm{J} 22141765+2534066$ & 2011.470 & $\mathrm{NIRC} 2 / \mathrm{N}$ & $H$ & 5 & $141.3 \pm 0.8$ & $304.09 \pm 0.05$ & $1.077 \pm 0.05$ & $9.68 \pm 0.02$ & $10.76 \pm 0.04$ & $41.7 \pm 0.8$ \\
\hline \multirow[t]{2}{*}{ GJ 4338 Bab } & $\mathrm{J} 23292258+4127522$ & 2010.628 & $\mathrm{NIRC} 2 / \mathrm{N}$ & $H$ & 3 & $46.7 \pm 0.9$ & $192.3 \pm 0.4$ & $0.27 \pm 0.06$ & $8.03 \pm 0.04$ & $8.30 \pm 0.04$ & $47 \pm 2$ \\
\hline & & 2011.470 & $\mathrm{NIRC} 2 / \mathrm{N}$ & $H$ & 4 & $78.2 \pm 0.9$ & $269.3 \pm 0.2$ & $0.43 \pm 0.06$ & $7.97 \pm 0.04$ & $8.40 \pm 0.05$ & $48 \pm 6$ \\
\hline GJ $4381 \mathrm{AB}$ & $\mathrm{J} 23574989+3837468$ & 2011.634 & $\mathrm{NIRC} 2 / \mathrm{N}$ & $H$ & 9 & $537.4 \pm 0.9$ & $247.365 \pm 0.016$ & $1.469 \pm 0.011$ & $8.30 \pm 0.02$ & $9.77 \pm 0.02$ & $38.2 \pm 0.4$ \\
\hline
\end{tabular}

Notes.

${ }^{a}$ Magnitude of the primary $\left(m_{\mathrm{A}}\right)$ decomposed from the integrated-light magnitude of the A and B components $\left(m_{\mathrm{AB}}\right)$ as follows: $m_{\mathrm{A}}=m_{\mathrm{AB}}+2.5 \log \left(1+10^{-\Delta \operatorname{mag} / 2.5}\right)$. Uncertainties are derived in a Monte Carlo

fashion. Unless otherwise noted, $J, H$, and $K_{S}$ magnitudes are from 2MASS (Skrutskie et al. 2006).

${ }^{\mathrm{b}}$ Magnitude of the companion $\left(m_{\mathrm{B}}\right)$ is computed from $\Delta$ mag and $m_{\mathrm{A}}$.

${ }^{c} Y$-band integrated-light magnitude is derived from the typical $Y-J$ color of dwarfs for the system spectral type from Rayner et al. (2009) and the integrated-light $J$-band magnitude.

${ }^{d} L^{\prime}$-band integrated-light magnitude is derived from the typical $K_{S}-L^{\prime}$ color of dwarfs for the system spectral type from Golimowski et al. (2004) and the integrated-light $K_{S}$-band magnitude.

${ }^{\mathrm{e}}$ Integrated-light $K_{S}$-band magnitude is converted to $K_{\mathrm{MKO}}$ using relations from Leggett et al. (2006). 

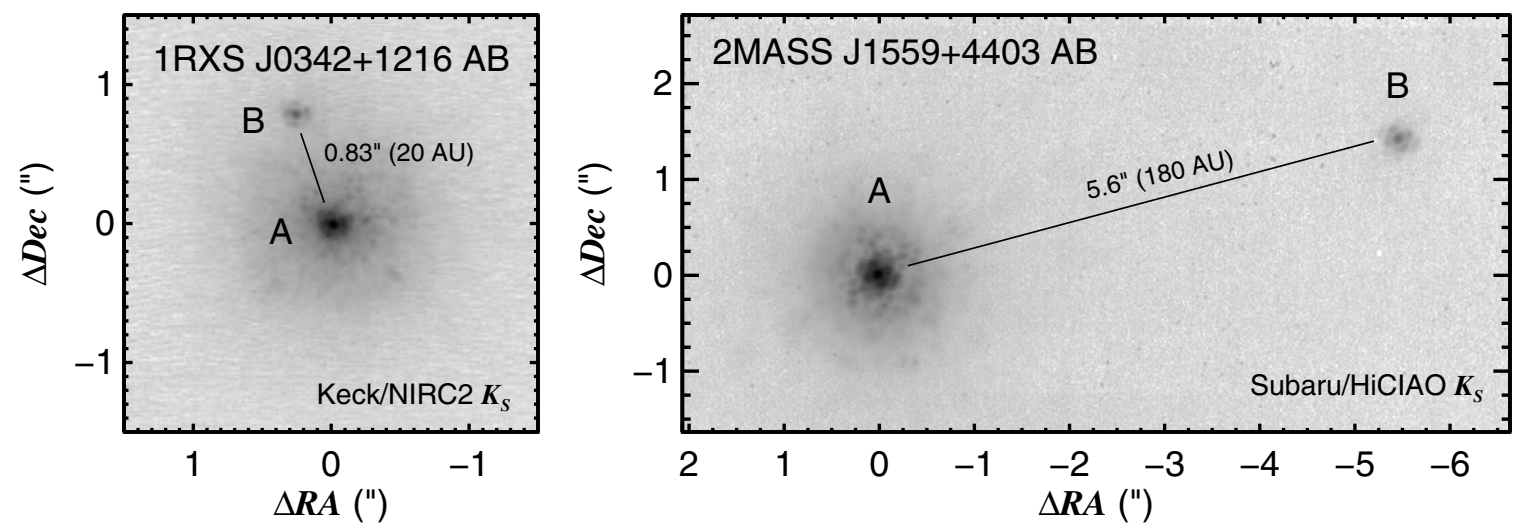

Figure 10. NIRC2 and HiCIAO images of two new brown dwarf companions identified in this survey. The inferred masses of 1 RXS J034231.8+121622 B (L0 \pm 1 ) and 2MASS J15594729+4403595 B (M8.0 \pm 0.5$)$ are $35 \pm 8 M_{\mathrm{Jup}}$ and $43 \pm 9 M_{\mathrm{Jup}}$, respectively. North is up and east is left.
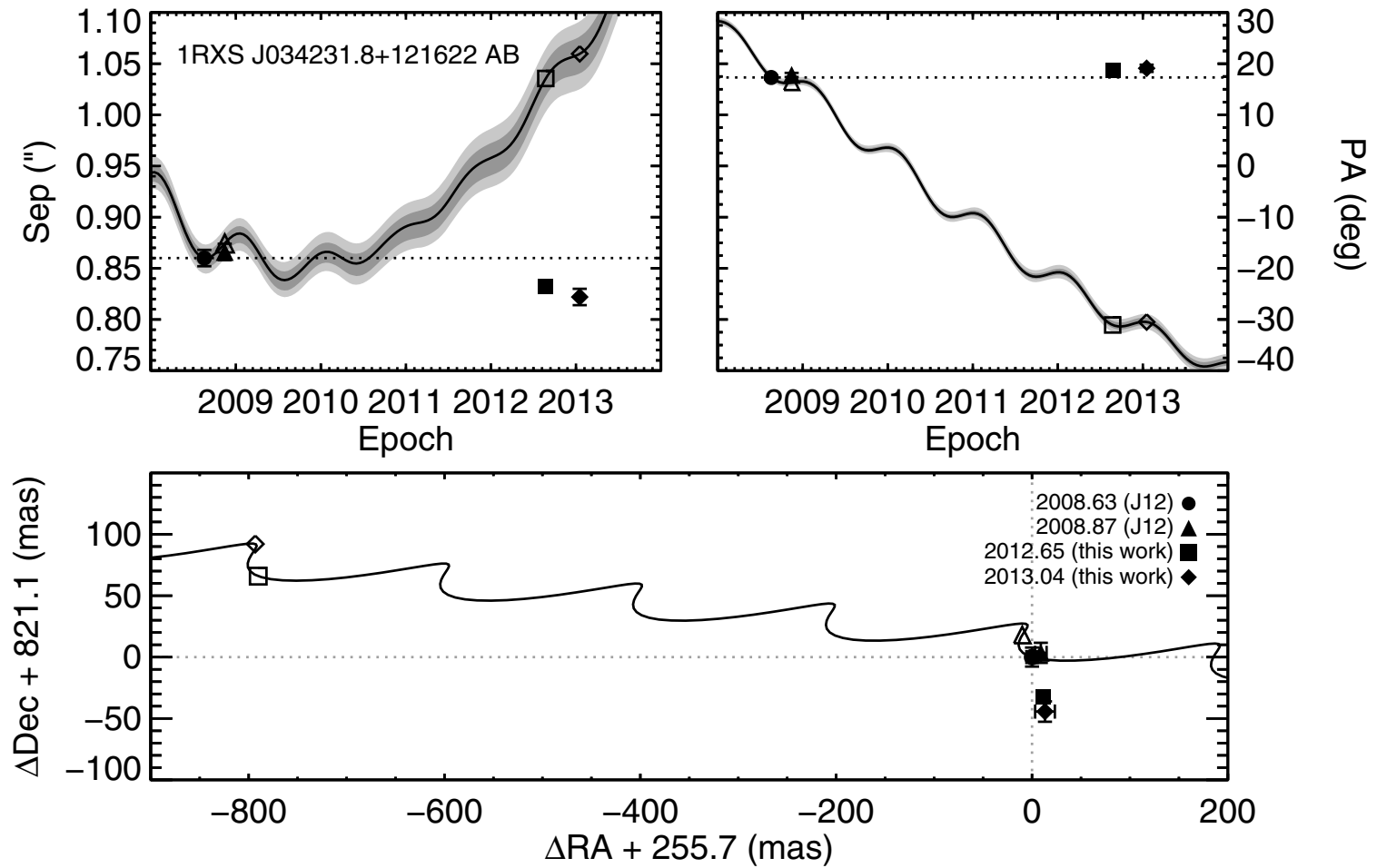

Figure 11. Test for physical association for the companion to 1RXS J034231.8+121622. The first two epochs in 2008 are from Janson et al. (2012). We confirm the companion is comoving and detect orbital motion with our AO imaging in 2012.

GALEX photometry (using Shkolnik et al. 2011 criteria), which is similar to known YMG members in $N U V-W 1$ color $(11.11 \pm$ 0.04 mag; see Rodriguez et al. 2013). Malo et al. (2013) found that 2MASS J15594729+4403595 A is a likely member of the 120 Myr AB Dor YMG based on its sky position, proper motion, and high-energy emission. Assuming group membership, they find a kinematic distance of $33 \pm 4 \mathrm{pc}$ to the primary and predict a radial velocity of $-28.9 \pm 1.8 \mathrm{~km} \mathrm{~s}^{-1}$.

E. L. Shkolnik et al. (in preparation) measure a radial velocity of $-19.6 \pm 0.6 \mathrm{~km} \mathrm{~s}^{-1}$ for 2MASS J15594729+4403595 as part of their follow-up efforts to kinematically associate nearby young stars with moving groups. Assuming the primary is not a single-lined spectroscopic binary, this velocity disagrees with the prediction by Malo et al. for AB Dor. In Figure 13 we show the partial kinematic constraints for distances between 20-60 pc. 2MASS J15594729+4403595 is consistent with $\beta$ Pic and Carina at $\sim 20 \mathrm{pc}$ and $\sim 50 \mathrm{pc}$. However, the $X Y Z$ positions disagree with all moving groups so we conclude that it is probably not a member of these known groups, which prevents precise age-dating through coevality with a young cluster. Additional radial velocities will help determine whether the primary is radial velocity stable and this measurement represents the systemic radial velocity.

Janson et al. (2012) imaged the system three times between 2008 and 2009, identifying 2MASS J15594729+4403595 B at 5.'6 (187 $\pm 23 \mathrm{AU})$ and confirming its physical association with the primary. Their $i^{\prime}$ - and $z$-band contrasts imply a spectral type of $\approx \mathrm{M} 8$ for the companion. We imaged the system in five filters at a single epoch in 2012 with HiCIAO (Figure 10). Our astrometry listed in Table 7 are consistent with that of Janson et al. and do not show signs of orbital motion (Figure 14).

We derive a $Y-J$ color of $0.80 \pm 0.05 \mathrm{mag}$ for $2 \mathrm{MASS}$ $\mathrm{J} 15594729+4403595 \mathrm{~B}$ in a similar fashion as for $1 \mathrm{RXS}$ J034231.8+121622 B. Compared to ultracool dwarfs in Rayner 

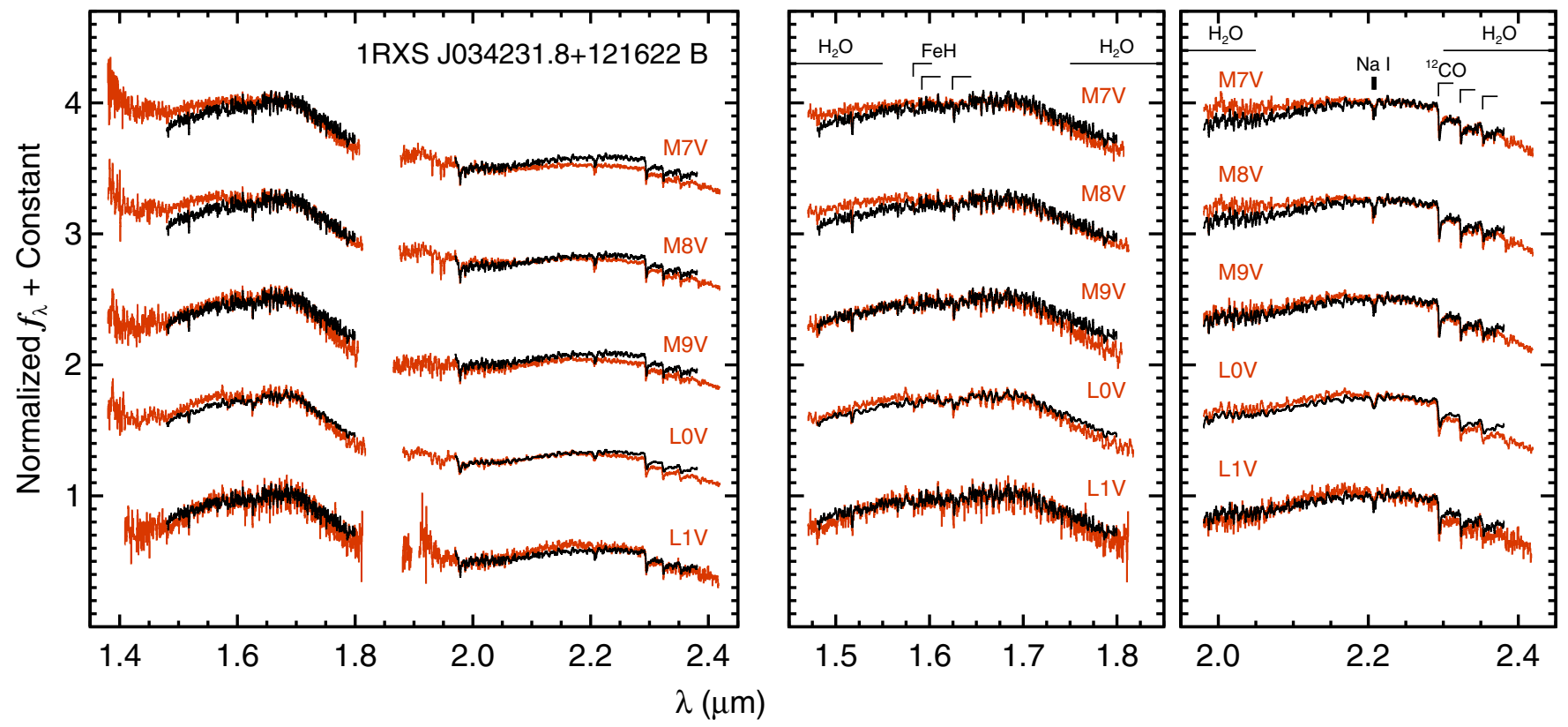

Figure 12. Our Keck/OSIRIS 1.4-2.4 $\mu \mathrm{m}$ spectrum of 1RXS J034231.8+121622 B compared to field objects from the IRTF SpeX Spectral Library. We adopt a spectral type of L0 \pm 1 for 1RXS J034231.8+121622 B. M7-M9 templates are listed in Figure 7. The L0 template is 2MASS J17312974+2721233 from Allers \& Liu (2013) and the L1 template is 2MASS J02081833+2542533 from Cushing et al. (2005). All spectra have been smoothed to a common resolving power of $R \approx 2000$ except for the L0 template, which has $R \approx 750$.
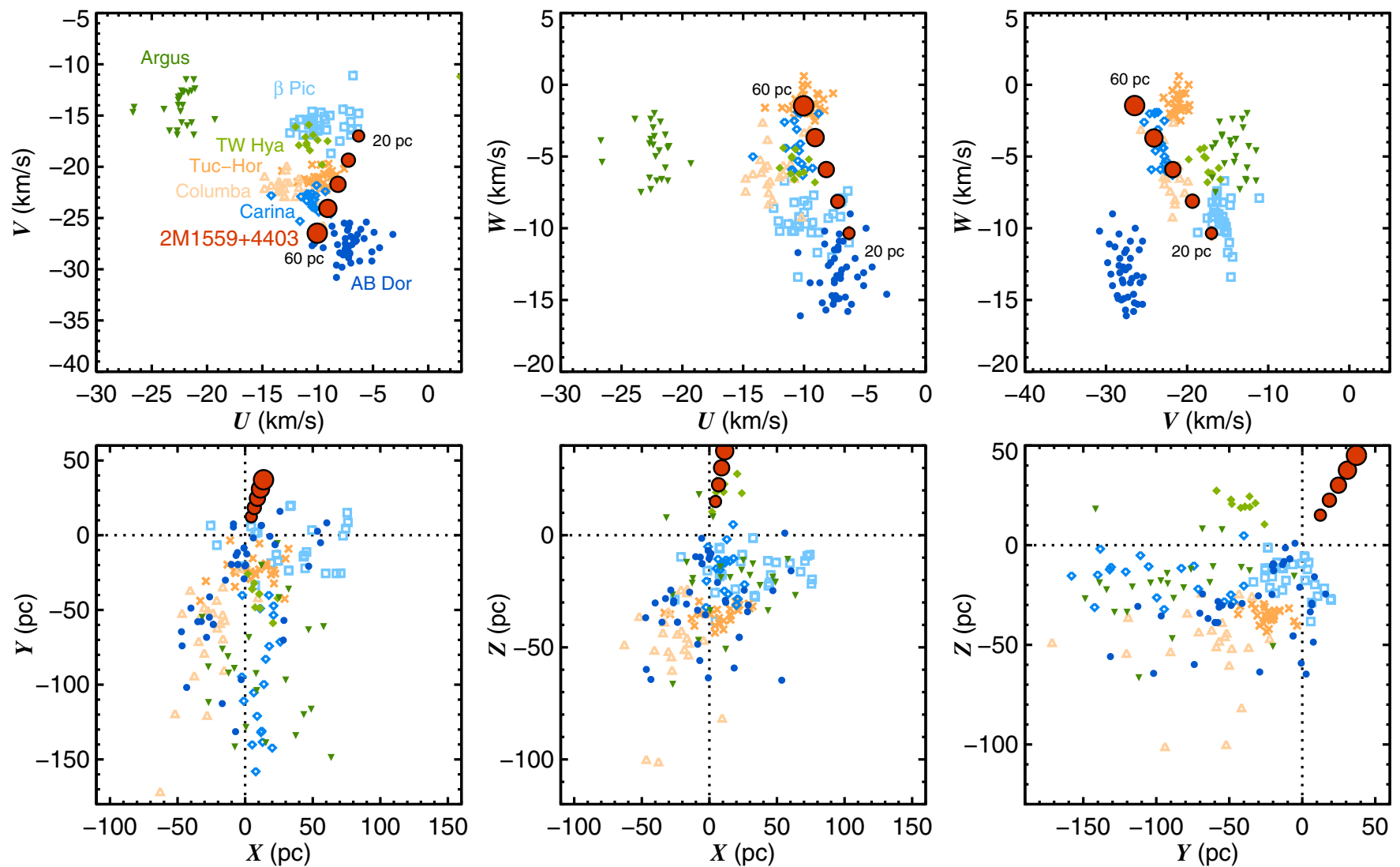

Figure 13. $U V W$ galactic velocities and $X Y Z$ space positions of 2 MASS J15594729+4403595 AB compared to nearby young moving groups from Torres et al. (2008). Although we lack a parallactic distance, the measured radial velocity to the primary enables partial kinematic constraints. 2MASS J15594729+4403595 AB is consistent with several moving groups in $U V W$ space for distances between $\sim 20-50 \mathrm{pc}$, but physically appears to be tens of parsecs from these same groups. Given this physical discrepancy, we find no convincing association with a known moving group. 

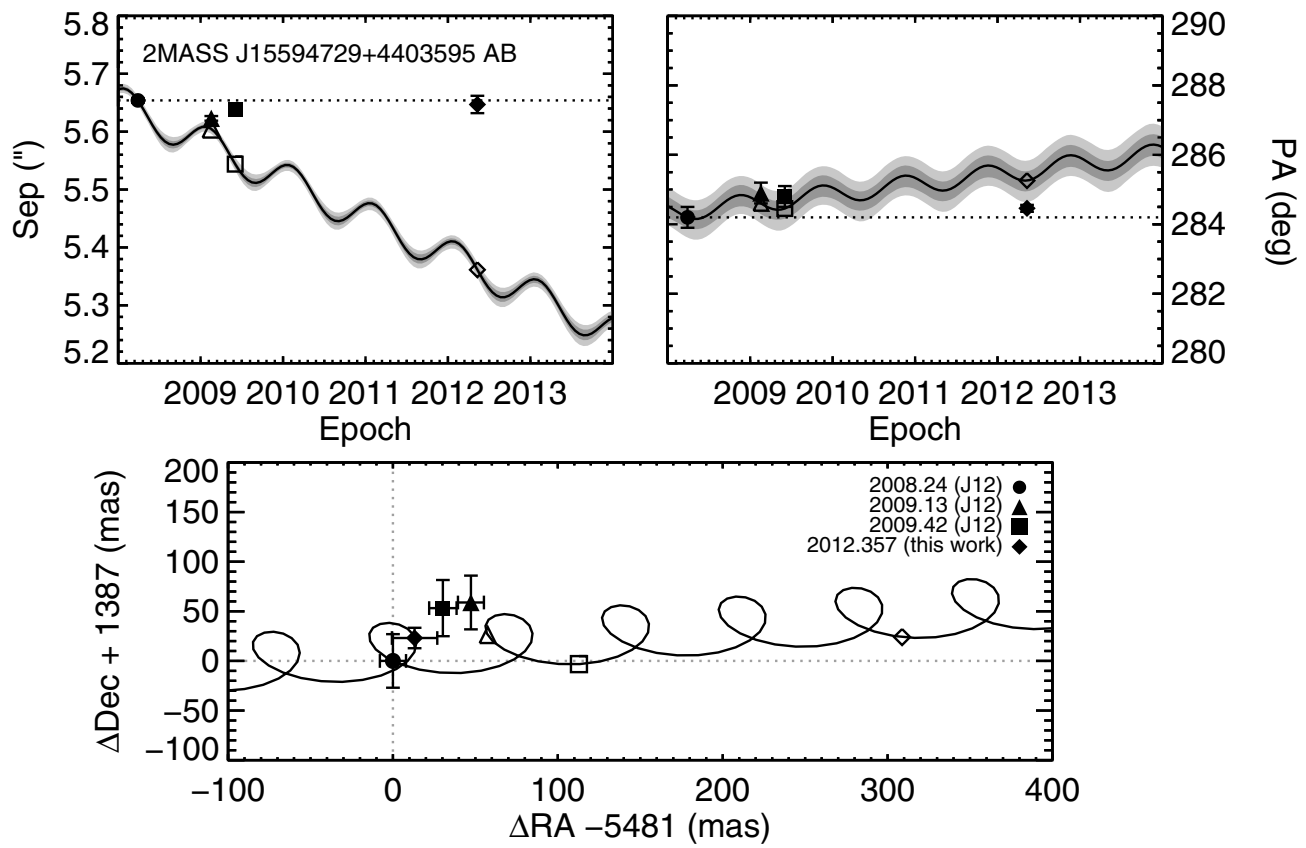

Figure 14. Relative astrometry of 2MASS J15594729+4403595 B compared to the expected background track of a stationary object (solid line). The first three epochs in 2008 and 2009 are from Janson et al. (2012). We verify the companion is unambiguously comoving from our AO imaging in 2012.
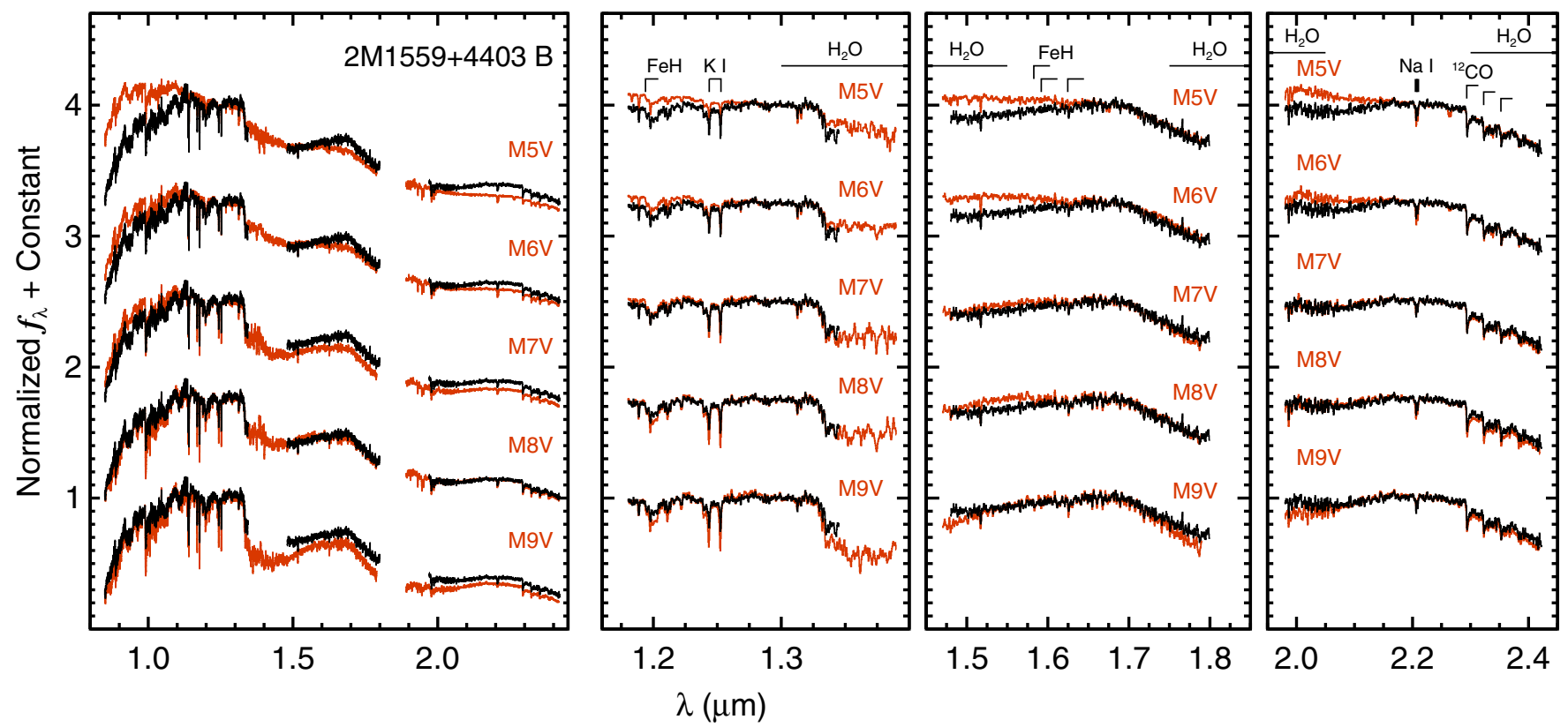

Figure 15. Our IRTF/SpeX SXD spectrum of 2MASS J15594729+4403595 B compared to field templates from the IRTF SpeX Spectral Library. 2MASS $\mathrm{J} 15594729+4403595 \mathrm{~B}$ is a good match to the field M8 template across all bandpasses, though the $H$-band shape is noticeably more angular in $2 \mathrm{MASS}$ $\mathrm{J} 15594729+4403595$ B. We adopt a spectral type of M8.0 \pm 0.5 . The comparison objects are the same as in Figure 7. All spectra have been smoothed to a common resolving power of $R \approx 1200$.

et al. (2009), we infer a photometric spectral type of M7.5 \pm 1 . Our $0.8-2.45 \mu \mathrm{m}$ IRTF/SpeX spectrum of 2 MASS $\mathrm{J} 15594729+4403595 \mathrm{~B}$ is shown in Figure 15. The best-fit spectral type across the entire spectrum is M8. M8, M7, and M7-M8 templates provide the best matches to field templates (Table 6). The gravity-insensitive index-based near-infrared classification schemes of Allers et al. (2007) and Slesnick et al. (2004) imply spectral types of M6.8 \pm 0.4, M7.6 \pm 1.1 , and M7.6 \pm 0.4 . Altogether we adopt a spectral type of M8.0 \pm 0.5 . The shallow $J$-band alkali lines and angular $H$ band are immediately clear and point to low surface gravity, which is supported by an "INT-G" gravity classification using the indices of Allers \& Liu (2013, see Table 6 and Figure 9). Indeed, 2MASS J15594729+4403595 B closely resembles the intermediate-gravity M8 object 2MASS J00192626+4614078 from Allers \& Liu (2013) in Figure 8.

Although low-gravity features in young brown dwarfs are not yet fully calibrated with empirical benchmarks, Allers \& Liu (2013) find that objects with intermediate-gravity spectra like 2MASS J15594729+4403595 B are most closely linked to brown dwarfs with ages between $~ 50-200$ Myr. Lacking 


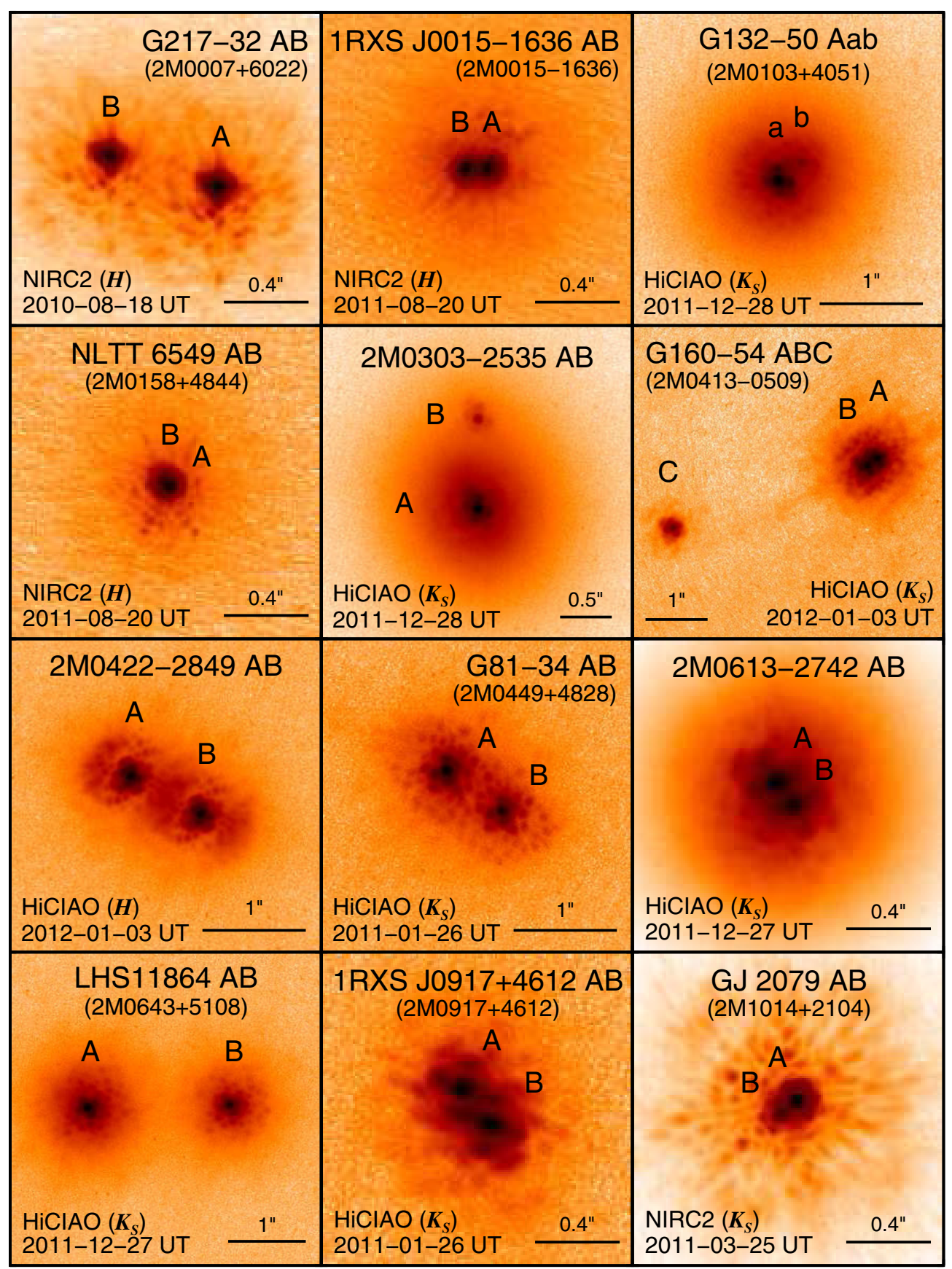

Figure 16. Binary stars detected in our survey. North is up and east is left.

a convincing association with a YMG, we adopt this spectroscopically inferred age for the system. The $H$-band photometric distance to the companion is $27 \pm 2 \mathrm{pc}$ using the absolute magnitude-spectral type relation from Dupuy \& Liu (2012). An $H$-band bolometric correction from Liu et al. (2010b) gives a bolometric luminosity of $-3.32 \pm 0.07 \mathrm{dex}$ which together with the system age implies a mass of $43 \pm 9 M_{\text {Jup }}$ for 2 MASS J15594729+4403595 B based on the evolutionary models of Burrows et al. (1997).

\subsection{Stellar Binaries and Multiples from the PALMS Survey}

Most of our targets have not been previously imaged with $\mathrm{AO}$ and, as expected, many were found to be close binaries
(Table 7 and Figures 16-18). Altogether 43 stars in our sample have stellar companions with projected separations less than $100 \mathrm{AU}$. We resolve 38 systems into binaries with angular separations from 50 mas to several arcseconds; 17 of these are separated by $<5 \mathrm{AU}$ in projection, 29 are separated by $<20 \mathrm{AU}$, and 37 of these are separated by $<100 \mathrm{AU}$. Among these, 27 are either new or spatially resolved for the first time in this work. An additional five targets not resolved in our data were found to be close spectroscopic binaries either from the literature or from E. L. Shkolnik et al. (in preparation). One additional target not resolved in our survey, LP 449-1 AB, was identified as a 50 mas binary by Riedel et al. (2014) with Hubble Space Telescope (HST) Fine Guidance Sensor interferometry. 


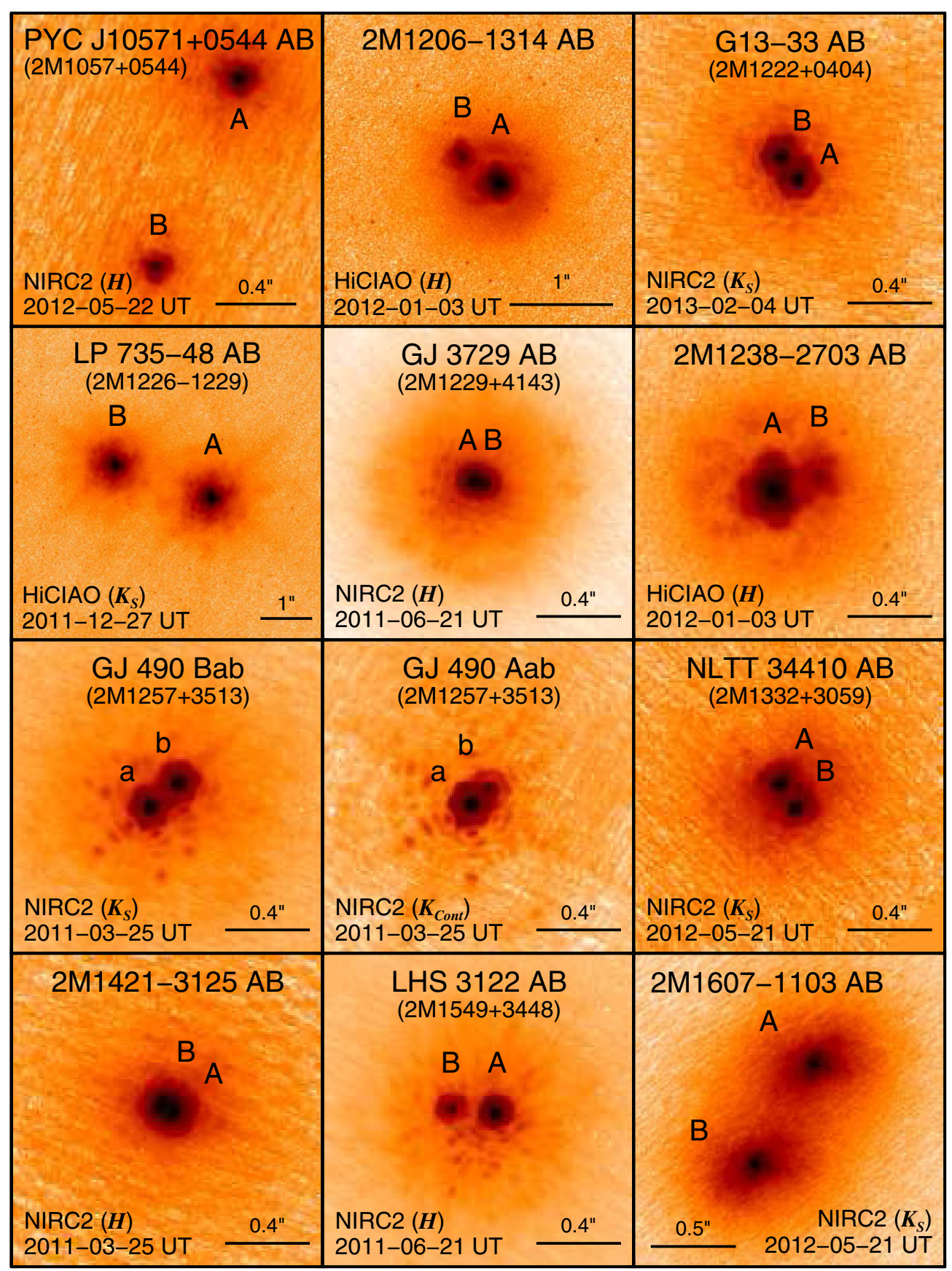

Figure 17. Binary stars detected in our survey (continued). North is up and east is left.

We intentionally vetted our initial target sample for previously known close binary systems which were found in heterogeneous studies. Since measuring the stellar companion mass function was not an original goal of this survey, we make no attempt to analyze the statistical properties of the multiples we uncovered. Nevertheless, we find that at least 43 out of 122 of our targets have stellar companions within $100 \mathrm{AU}$, implying a minimum companion frequency of $>35.4 \% \pm 4.3 \%$. This agrees well with the established close companion fraction of $33 \% \pm 5 \%$ (Duchêne \& Kraus 2013).

Several binaries were not seen in our first epoch of deep imaging and were only resolved in follow-up observations of wide planet candidates. These data are not incorporated into our statistical analysis so as to maintain a homogeneous sample of single stars. Note that the closest binaries with projected separations less than a few AU will yield dynamical masses on short timescales.

Seven targets in our sample form higher order hierarchical multiple systems. Five of these are triple systems (G 160-54 ABC, GJ 9652 Aab + GJ 9652 B, 2MASS J19560294-3207186 $\mathrm{AB}+$ TYC 7443-1102-1, GJ $4185 \mathrm{Aab}+\mathrm{GJ} 4186$ B, GJ $4338 \mathrm{Bab}+$ GJ $4337 \mathrm{~A})$ and two make up quadruple systems (G 132-50 Aab + G 132-51 Bab, GJ 490 Aab+ GJ $490 \mathrm{Bab})$. 


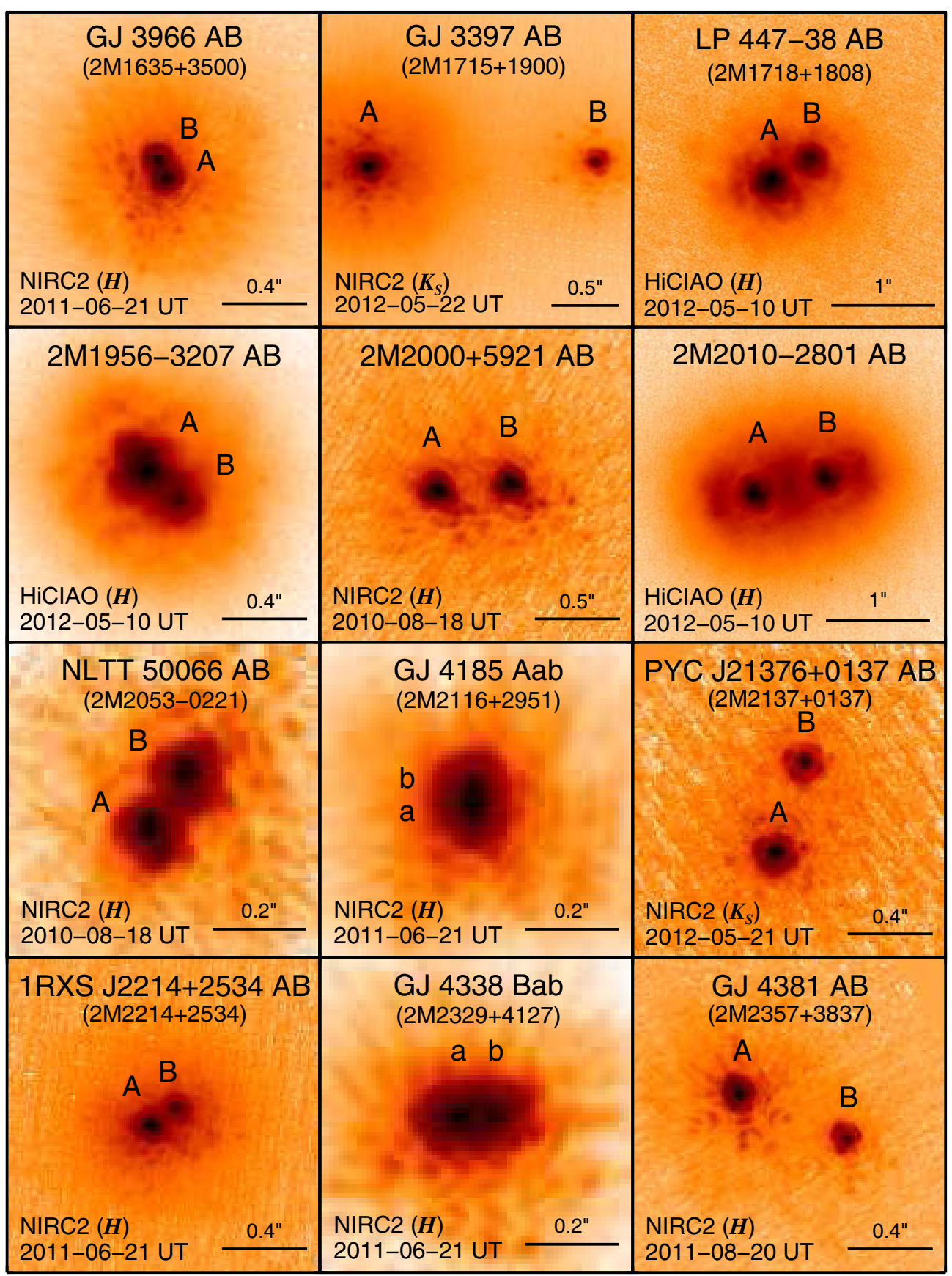

Figure 18. Binary stars detected in our survey (continued). North is up and east is left.

\subsection{Candidate Planets from the PALMS Survey}

Altogether 167 faint point sources were identified around 45 stars (singles and binaries) in our deep imaging (Figure 19). 42 of these candidates are in the crowded low-galactic latitude field surrounding the single-line spectroscopic binary GJ 9652 A. Astrometry and relative photometry are listed in Table 8. Background stars are distinguished from comoving gravitationally bound companions using two or more epochs of follow-up imaging, with a prioritization for those at small projected separations under $100 \mathrm{AU}$. In some cases, candidates are visible in archival wide-field imaging surveys like the Digitized Sky Survey (first and second generations), the Sloan Digital Sky Survey (Abazajian et al. 2009), and 2MASS (Skrutskie et al. 2006) and were rejected if their astrometry and/or colors were inconsistent with cool, comoving companions. Inevitably our second-epoch observations uncovered candidates not seen in our first epoch data, but most of these reside at wide separations beyond the main region of interest $(\sim 10-100 \mathrm{AU})$.

For candidates with multiple epochs of astrometry we calculate a reduced chi-squared value for a background scenario, $\chi_{v, B G}^{2}$, and a common proper motion scenario, $\chi_{v, \mathrm{CPM}}^{2} \cdot$ Here

$$
\chi_{\nu}^{2}=\frac{1}{v} \sum_{i=1}^{N-1}\left(\frac{\left(\theta_{\text {meas }, i}-\theta_{\text {pred }, i}\right)^{2}}{\sigma_{\theta, \text { meas }, i}^{2}+\sigma_{\theta, \text { pred }, i}^{2}}+\frac{\left(\rho_{\text {meas }, i}-\rho_{\text {pred }, i}\right)^{2}}{\sigma_{\rho, \text { meas }, i}^{2}+\sigma_{\rho, \text { pred }, i}^{2}}\right),
$$


Table 8

Candidate Companions

\begin{tabular}{|c|c|c|c|c|c|c|c|c|c|c|c|c|}
\hline \multirow[b]{2}{*}{ Name } & \multirow[b]{2}{*}{ Cand. } & \multirow[b]{2}{*}{$\begin{array}{l}\text { UT Date } \\
\text { (Y-M-D) }\end{array}$} & \multirow[b]{2}{*}{ Epoch } & \multirow[b]{2}{*}{ Instrument $^{\mathrm{a}}$} & \multirow[b]{2}{*}{ Filt. } & \multirow[b]{2}{*}{$\begin{array}{c}\text { Contrast } \\
\text { (mag) }\end{array}$} & \multicolumn{2}{|c|}{ Measured Position } & \multicolumn{2}{|c|}{ Background Position } & \multirow[b]{2}{*}{$\begin{array}{l}\text { Proj. Sep. } \\
\quad(\mathrm{AU})\end{array}$} & \multirow[b]{2}{*}{ Comp.? } \\
\hline & & & & & & & $\begin{array}{c}\rho \\
\left({ }^{\prime \prime}\right)\end{array}$ & $\begin{array}{l}\text { P.A. } \\
\left({ }^{\circ}\right)\end{array}$ & $\begin{array}{c}\rho \\
\left({ }^{\prime \prime}\right)\end{array}$ & $\begin{array}{l}\text { P.A. } \\
\left({ }^{\circ}\right)\end{array}$ & & \\
\hline GJ $3030 \mathrm{AB}$ & $\mathrm{CC} 1$ & $2011 \operatorname{Dec} 27$ & 2011.9862 & HiCIAO & $H$ & $9.65 \pm 0.60$ & $5.411 \pm 0.020$ & $67.58 \pm 0.25$ & & & 135.3 & $\mathrm{BG}^{\mathrm{d}}$ \\
\hline GJ $3030 \mathrm{AB}$ & $\mathrm{CC} 1$ & 2013 Aug 17 & 2013.6259 & $\mathrm{NIRC} 2 / \mathrm{W}$ & $K_{S}$ & $\ldots \mathrm{c}$ & $5.139 \pm 0.074$ & $65.59 \pm 0.20$ & $5.029 \pm 0.041$ & $65.56 \pm 0.36$ & 128.5 & $\mathrm{BG}$ \\
\hline GJ $3030 \mathrm{AB}$ & $\mathrm{CC} 2$ & 2011 Dec 27 & 2011.9862 & HiCIAO & $H$ & $7.88 \pm 0.59$ & $5.785 \pm 0.021$ & $1.01 \pm 0.24$ & & & 144.6 & $B G^{d}$ \\
\hline GJ $3030 \mathrm{AB}$ & $\mathrm{CC} 2$ & 2013 Aug 17 & 2013.6259 & $\mathrm{NIRC} 2 / \mathrm{W}$ & $K_{S}$ & $\ldots \mathrm{c}$ & $5.894 \pm 0.074$ & $357.88 \pm 0.20$ & $5.810 \pm 0.033$ & $356.82 \pm 0.39$ & 147.4 & BG \\
\hline NLTT 1875 & $\mathrm{CC} 1$ & 2010 Aug 18 & 2010.6283 & $\mathrm{NIRC} 2 / \mathrm{N}$ & $H$ & $8.57 \pm 0.16$ & $5.588 \pm 0.003$ & $339.65 \pm 0.20$ & $\ldots$ & $\ldots$ & 214.0 & $\ldots$ \\
\hline NLTT 1875 & $\mathrm{CC} 1$ & 2011 Aug 20 & 2011.6339 & $\mathrm{NIRC} 2 / \mathrm{N}$ & $H$ & $8.78 \pm 0.16$ & $5.687 \pm 0.003$ & $337.35 \pm 0.20$ & $5.673 \pm 0.004$ & $337.52 \pm 0.19$ & 217.8 & $\mathrm{BG}$ \\
\hline NLTT 1875 & $\mathrm{CC} 2$ & 2010 Aug 18 & 2010.6283 & $\mathrm{NIRC} 2 / \mathrm{N}$ & $H$ & $9.69 \pm 0.16$ & $3.562 \pm 0.003$ & $331.45 \pm 0.20$ & $\ldots$ & $\ldots$ & 136.4 & $\ldots$ \\
\hline NLTT 1875 & $\mathrm{CC} 2$ & 2011 Aug 20 & 2011.6339 & $\mathrm{NIRC} 2 / \mathrm{N}$ & $H$ & $10.05 \pm 0.21$ & $3.690 \pm 0.003$ & $328.32 \pm 0.20$ & $3.677 \pm 0.004$ & $328.40 \pm 0.20$ & 141.3 & BG \\
\hline NLTT 1875 & $\mathrm{CC} 3$ & 2010 Aug 18 & 2010.6283 & NIRC2/N & $H$ & $11.51 \pm 0.30$ & $5.096 \pm 0.003$ & $249.85 \pm 0.20$ & & & 195.2 & \\
\hline NLTT 1875 & $\mathrm{CC} 3$ & 2011 Aug 20 & 2011.6339 & $\mathrm{NIRC} 2 / \mathrm{N}$ & $H$ & $11.75 \pm 0.92$ & $5.312 \pm 0.003$ & $250.68 \pm 0.20$ & $5.306 \pm 0.004$ & $250.72 \pm 0.20$ & 203.4 & BG \\
\hline NLTT 1875 & $\mathrm{CC} 4$ & 2010 Aug 18 & 2010.6283 & $\mathrm{NIRC} 2 / \mathrm{N}$ & $H$ & $8.59 \pm 0.16$ & $2.612 \pm 0.003$ & $210.23 \pm 0.20$ & $\ldots$ & $\ldots$ & 100.0 & $\ldots$ \\
\hline NLTT 1875 & $\mathrm{CC} 4$ & 2011 Aug 20 & 2011.6339 & NIRC2/N & $H$ & $8.53 \pm 0.16$ & $2.718 \pm 0.003$ & $214.37 \pm 0.20$ & $2.729 \pm 0.004$ & $214.33 \pm 0.19$ & 104.1 & BG \\
\hline NLTT 1875 & $\mathrm{CC} 5$ & 2010 Aug 18 & 2010.6283 & $\mathrm{NIRC} 2 / \mathrm{N}$ & $H$ & $9.24 \pm 0.16$ & $3.839 \pm 0.003$ & $191.60 \pm 0.20$ & & $\ldots$ & 147.0 & $\ldots$ \\
\hline NLTT 1875 & $\mathrm{CC} 5$ & 2011 Aug 20 & 2011.6339 & $\mathrm{NIRC} 2 / \mathrm{N}$ & $H$ & $9.45 \pm 0.17$ & $3.874 \pm 0.003$ & $194.74 \pm 0.20$ & $3.887 \pm 0.004$ & $194.84 \pm 0.19$ & 148.4 & BG \\
\hline NLTT 1875 & CC6 & 2010 Aug 18 & 2010.6283 & $\mathrm{NIRC} 2 / \mathrm{N}$ & $H$ & $10.25 \pm 0.17$ & $5.939 \pm 0.003$ & $169.58 \pm 0.20$ & & & 227.5 & \\
\hline NLTT 1875 & CC6 & 2011 Aug 20 & 2011.6339 & $\mathrm{NIRC} 2 / \mathrm{N}$ & $H$ & $10.32 \pm 0.26$ & $5.884 \pm 0.003$ & $171.54 \pm 0.20$ & $5.899 \pm 0.004$ & $171.72 \pm 0.20$ & 225.3 & BG \\
\hline G 132-50 Aab & $\mathrm{CC} 1$ & 2011 Dec 28 & 2011.9889 & HiCIAO & $K_{S}$ & $8.96 \pm 0.54$ & $11.897 \pm 0.042$ & $335.01 \pm 0.17$ & $\ldots$ & $\ldots$ & 355.7 & $\mathrm{BG}^{\mathrm{d}}$ \\
\hline G 172-56 & $\mathrm{CC} 1$ & 2010 Aug 17 & 2010.6257 & $\mathrm{NIRC} / \mathrm{N}$ & $H$ & $12.67 \pm 0.84$ & $4.534 \pm 0.003$ & $33.54 \pm 0.20$ & $\ldots$ & $\ldots$ & 81.6 & SE \\
\hline G $172-56$ & $\mathrm{CC} 2$ & 2010 Aug 17 & 2010.6257 & $\mathrm{NIRC} 2 / \mathrm{N}$ & $H$ & $12.15 \pm 0.60$ & $5.061 \pm 0.003$ & $143.38 \pm 0.20$ & $\ldots$ & $\ldots$ & 91.1 & $B G^{e}$ \\
\hline G 172-56 & $\mathrm{CC} 3$ & 2010 Aug 17 & 2010.6257 & $\mathrm{NIRC} 2 / \mathrm{N}$ & $H$ & $11.28 \pm 0.35$ & $6.590 \pm 0.003$ & $160.71 \pm 0.20$ & $\ldots$ & $\ldots$ & 118.6 & SE \\
\hline G 271-110 & $\mathrm{CC} 1$ & 2010 Aug 18 & 2010.6284 & $\mathrm{NIRC} 2 / \mathrm{N}$ & $H$ & $13.82 \pm 0.98$ & $6.662 \pm 0.003$ & $23.18 \pm 0.20$ & $\ldots$ & $\ldots$ & 159.9 & SE \\
\hline NLTT 6549 AB & $\mathrm{CC} 1$ & 2011 Aug 20 & 2011.6342 & $\mathrm{NIRC} 2 / \mathrm{N}$ & $H$ & $15.09 \pm 0.91$ & $3.803 \pm 0.003$ & $222.87 \pm 0.20$ & $\ldots$ & $\ldots$ & 167.3 & $\mathrm{SE}$ \\
\hline GJ 3136 & $\mathrm{CC} 1$ & 2011 Jan 27 & 2011.0718 & HiCIAO & $K_{S}$ & $6.90 \pm 0.20$ & $9.846 \pm 0.036$ & $302.19 \pm 0.18$ & $\ldots$ & $\ldots$ & 147.7 & $\mathrm{BG}^{\mathrm{d}}$ \\
\hline 1RXS J022735.8+471021 & $\mathrm{CC} 1$ & 2010 Aug 17 & 2010.6256 & $\mathrm{NIRC} 2 / \mathrm{N}$ & $H$ & $13.38 \pm 0.76$ & $3.244 \pm 0.003$ & $233.25 \pm 0.20$ & $\ldots$ & $\ldots$ & 88.9 & SE \\
\hline 1RXS J022735.8+471021 & $\mathrm{CC} 2$ & $2013 \mathrm{Feb} 4$ & 2013.0938 & $\mathrm{NIRC} 2 / \mathrm{N}$ & $H$ & $10.50 \pm 0.23$ & $4.779 \pm 0.003$ & $163.80 \pm 0.20$ & $\ldots$ & $\ldots$ & 130.9 & SE \\
\hline 1RXS J022735.8+471021 & $\mathrm{CC} 3$ & $2013 \mathrm{Feb} 4$ & 2013.0938 & $\mathrm{NIRC} 2 / \mathrm{N}$ & $H$ & $9.12 \pm 0.19$ & $5.660 \pm 0.003$ & $155.87 \pm 0.20$ & $\ldots$ & $\ldots$ & 155.1 & SE \\
\hline 1RXS J023138.7+445640 & $\mathrm{CC} 1$ & 2010 Aug 18 & 2010.6285 & $\mathrm{NIRC} 2 / \mathrm{N}$ & $H$ & $8.57 \pm 0.16$ & $7.438 \pm 0.003$ & $269.99 \pm 0.20$ & $\ldots$ & $\ldots$ & 119.0 & SE \\
\hline G 75-35 & $\mathrm{CC} 1$ & 2012 Jan 3 & 2012.0062 & HiCIAO & $H$ & $10.77 \pm 0.45$ & $11.762 \pm 0.024$ & $255.20 \pm 0.17$ & $\cdots$ & $\cdots$ & 188.2 & $\mathrm{BG}^{\mathrm{d}}$ \\
\hline LP $247-13$ & $\mathrm{CC} 1$ & 2011 Jan 26 & 2011.0692 & HiCIAO & $K_{S}$ & $11.09 \pm 1.17$ & $1.799 \pm 0.020$ & $341.64 \pm 0.63$ & $\ldots$ & $\ldots$ & 61.9 & $\ldots$ \\
\hline LP $247-13$ & $\mathrm{CC} 1$ & 2011 Aug 20 & 2011.6340 & $\mathrm{NIRC} 2 / \mathrm{N}$ & $H$ & $10.92 \pm 0.21$ & $1.894 \pm 0.003$ & $338.21 \pm 0.20$ & $1.900 \pm 0.021$ & $337.11 \pm 0.62$ & 65.1 & $\mathrm{BG}$ \\
\hline LP $247-13$ & $\mathrm{CC} 1$ & 2011 Aug 20 & 2011.6340 & $\mathrm{NIRC} 2 / \mathrm{N}$ & $K_{S}$ & $13.13 \pm 0.85$ & $1.903 \pm 0.003$ & $338.20 \pm 0.20$ & $1.900 \pm 0.021$ & $337.11 \pm 0.62$ & 65.4 & BG \\
\hline LP 247-13 & $\mathrm{CC} 1$ & 2012 Jan 14 & 2012.0365 & $\mathrm{NIRC} 2 / \mathrm{N}$ & $H$ & $11.27 \pm 0.17$ & $1.941 \pm 0.003$ & $337.87 \pm 0.20$ & $1.957 \pm 0.022$ & $336.80 \pm 0.63$ & 66.8 & $\mathrm{BG}$ \\
\hline LP $247-13$ & $\mathrm{CC} 1$ & 2012 Jan 14 & 2012.0365 & $\mathrm{NIRC} 2 / \mathrm{N}$ & $K$ & $11.15 \pm 0.15$ & $1.956 \pm 0.003$ & $338.17 \pm 0.20$ & $1.957 \pm 0.022$ & $336.80 \pm 0.63$ & 67.3 & BG \\
\hline LP 247-13 & $\mathrm{CC} 2$ & 2011 Jan 26 & 2011.0692 & HiCIAO & $K_{S}$ & $11.12 \pm 1.16$ & $6.692 \pm 0.028$ & $317.87 \pm 0.22$ & $\ldots$ & - & 230.2 & SE \\
\hline GJ 3287 & $\mathrm{CC} 1$ & $2013 \mathrm{Feb} 4$ & 2013.0939 & $\mathrm{NIRC} 2 / \mathrm{N}$ & $K_{S}$ & $9.29 \pm 0.22$ & $4.239 \pm 0.003$ & $184.13 \pm 0.20$ & & 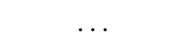 & 95.4 & SE \\
\hline NLTT13844 & $\mathrm{CC} 1$ & 2012 Oct 8 & 2012.7710 & $\mathrm{NIRC} 2 / \mathrm{N}$ & $H$ & $10.24 \pm 0.22$ & $3.870 \pm 0.003$ & $353.14 \pm 0.20$ & $\ldots$ & $\ldots$ & 81.3 & $\ldots$ \\
\hline NLTT13844 & $\mathrm{CC} 1$ & 2013 Jan 17 & 2013.0448 & $\mathrm{NIRC} 2 / \mathrm{N}$ & $K$ & $9.70 \pm 0.32$ & $4.060 \pm 0.003$ & $353.01 \pm 0.20$ & $4.053 \pm 0.004$ & $352.97 \pm 0.19$ & 85.3 & BG \\
\hline NLTT13844 & $\mathrm{CC} 2$ & 2012 Oct 8 & 2012.7710 & $\mathrm{NIRC} 2 / \mathrm{N}$ & $H$ & $10.74 \pm 0.31$ & $2.832 \pm 0.003$ & $165.37 \pm 0.20$ & $\ldots$ & $\ldots$ & 59.5 & $\ldots$ \\
\hline NLTT13844 & $\mathrm{CC} 2$ & 2013 Jan 17 & 2013.0448 & $\mathrm{NIRC} 2 / \mathrm{N}$ & $K$ & $10.20 \pm 0.64$ & $2.647 \pm 0.003$ & $165.03 \pm 0.20$ & $2.649 \pm 0.004$ & $165.10 \pm 0.23$ & 55.6 & BG \\
\hline 1RXS J045101.0+312734 & $\mathrm{CC} 1$ & 2011 Jan 27 & 2011.0721 & HiCIAO & $K_{S}$ & $4.93 \pm 0.26$ & $10.410 \pm 0.038$ & $282.58 \pm 0.18$ & $\ldots$ & $\ldots$ & 135.3 & $\cdots$ \\
\hline 1RXS J045101.0+312734 & $\mathrm{CC} 1$ & 2011 Dec 28 & 2011.9894 & HiCIAO & $K_{S}$ & $5.81 \pm 0.41$ & $10.615 \pm 0.038$ & $282.66 \pm 0.18$ & $10.623 \pm 0.041$ & $282.48 \pm 0.18$ & 138.0 & BG \\
\hline 1RXS J045101.0+312734 & $\mathrm{CC} 1$ & 2012 Jan 3 & 2012.0068 & HiCIAO & $K_{S}$ & $5.24 \pm 0.06$ & $10.623 \pm 0.038$ & $282.55 \pm 0.18$ & $10.618 \pm 0.040$ & $282.50 \pm 0.18$ & 138.1 & BG \\
\hline 1RXS J045101.0+312734 & $\mathrm{CC} 2$ & 2011 Jan 27 & 2011.0721 & HiCIAO & $K_{S}$ & $10.20 \pm 0.78$ & $9.393 \pm 0.035$ & $281.59 \pm 0.18$ & $\ldots$ & $\ldots$ & 122.1 & $\ldots$ \\
\hline 1RXS J045101.0+312734 & $\mathrm{CC} 2$ & 2012 Jan 3 & 2012.0068 & HiCIAO & $K_{S}$ & $10.28 \pm 1.08$ & $9.622 \pm 0.036$ & $281.59 \pm 0.18$ & $9.603 \pm 0.037$ & $281.52 \pm 0.19$ & 125.1 & BG \\
\hline
\end{tabular}


Table 8

\begin{tabular}{|c|c|c|c|c|c|c|c|c|c|c|c|c|}
\hline \multirow[b]{2}{*}{ Name } & \multirow[b]{2}{*}{ Cand. } & \multirow[b]{2}{*}{$\begin{array}{l}\text { UT Date } \\
\text { (Y-M-D) }\end{array}$} & \multirow[b]{2}{*}{ Epoch } & \multirow[b]{2}{*}{ Instrument $^{\mathrm{a}}$} & \multirow[b]{2}{*}{ Filt. } & \multirow[b]{2}{*}{$\begin{array}{l}\text { Contrast } \\
\text { (mag) }\end{array}$} & \multicolumn{2}{|c|}{ Measured Position } & \multicolumn{2}{|c|}{ Background Position } & \multirow[b]{2}{*}{$\begin{array}{l}\text { Proj. Sep. } \\
\text { (AU) }\end{array}$} & \multirow[b]{2}{*}{ Comp.? ${ }^{\mathrm{b}}$} \\
\hline & & & & & & & $\begin{array}{c}\rho \\
\left({ }^{\prime \prime}\right)\end{array}$ & $\begin{array}{l}\text { P.A. } \\
\left({ }^{\circ}\right)\end{array}$ & $\begin{array}{c}\rho \\
\left({ }^{\prime \prime}\right)\end{array}$ & $\begin{array}{l}\text { P.A. } \\
\left({ }^{\circ}\right)\end{array}$ & & \\
\hline 1RXS J055446.0+105559 & $\mathrm{CC} 1$ & 2013 Feb 4 & 2013.0940 & $\mathrm{NIRC} 2 / \mathrm{N}$ & $K_{S}$ & $14.38 \pm 0.92$ & $3.770 \pm 0.003$ & $34.37 \pm 0.20$ & $\ldots$ & $\ldots$ & 95.8 & SE \\
\hline 1RXS J055446.0+105559 & $\mathrm{CC} 2$ & 2013 Feb 4 & 2013.0940 & $\mathrm{NIRC} 2 / \mathrm{N}$ & $K_{S}$ & $14.04 \pm 1.07$ & $6.443 \pm 0.003$ & $107.95 \pm 0.20$ & $\ldots$ & $\ldots$ & 163.7 & SE \\
\hline 1RXS J055446.0+105559 & $\mathrm{CC} 3$ & 2013 Feb 4 & 2013.0940 & NIRC $2 / \mathrm{N}$ & $K_{S}$ & $11.86 \pm 0.15$ & $4.920 \pm 0.003$ & $148.42 \pm 0.20$ & $\begin{array}{l}\cdots \\
\cdots\end{array}$ & $\cdots$ & 125.0 & SE \\
\hline 1RXS J055446.0+105559 & $\mathrm{CC} 4$ & 2013 Feb 4 & 2013.0940 & $\mathrm{NIRC} 2 / \mathrm{N}$ & $K_{S}$ & $11.65 \pm 0.14$ & $4.991 \pm 0.003$ & $171.31 \pm 0.20$ & $\ldots$ & $\ldots$ & 126.8 & SE \\
\hline 1RXS J055446.0+105559 & $\mathrm{CC} 5$ & 2013 Feb 4 & 2013.0940 & $\mathrm{NIRC} 2 / \mathrm{N}$ & $K_{S}$ & $11.48 \pm 0.15$ & $6.469 \pm 0.003$ & $191.22 \pm 0.20$ & $\ldots$ & $\ldots$ & 164.3 & SE \\
\hline $\mathrm{AP} \mathrm{Col}$ & $\mathrm{CC} 1$ & 2011 Dec 27 & 2011.9867 & HiCIAO & $K_{S}$ & $11.16 \pm 1.16$ & $6.899 \pm 0.029$ & $141.80 \pm 0.21$ & $\ldots$ & $\ldots$ & 57.9 & $\ldots$ \\
\hline AP Col & $\mathrm{CC} 1$ & 2012 Jan 3 & 2012.0065 & HiCIAO & $H$ & $11.30 \pm 0.10$ & $6.853 \pm 0.021$ & $142.10 \pm 0.22$ & $6.914 \pm 0.028$ & $141.75 \pm 0.21$ & 57.5 & \\
\hline AP Col & $\mathrm{CC} 1$ & 2012 Oct 8 & 2012.7711 & NIRC2/W & $H$ & $\ldots{ }^{c}$ & $7.040 \pm 0.074$ & $145.09 \pm 0.20$ & $7.089 \pm 0.030$ & $144.38 \pm 0.22$ & 59.1 & BG \\
\hline $\mathrm{AP} \mathrm{Col}$ & $\mathrm{CC} 2$ & 2012 Oct 8 & 2012.7711 & NIRC2/W & $H$ & $\ldots c$ & $16.649 \pm 0.076$ & $171.49 \pm 0.20$ & 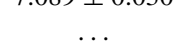 & $\begin{array}{c}1+50.0 .22 \\
\ldots\end{array}$ & 139.7 & SE \\
\hline AP Col & $\mathrm{CC} 3$ & 2012 Oct 8 & 2012.7711 & $\mathrm{NIRC} 2 / \mathrm{W}$ & $H$ & $\ldots c$ & $18.780 \pm 0.076$ & $115.67 \pm 0.20$ & $\ldots$ & $\ldots$ & 157.6 & SE \\
\hline GJ 3395 & $\mathrm{CC} 1$ & 2011 Jan 26 & 2011.0695 & HiCIAO & $K_{S}$ & $10.12 \pm 0.61$ & $11.833 \pm 0.042$ & $203.89 \pm 0.17$ & $\ldots$ & $\ldots$ & 331.3 & $\mathrm{BG}^{\mathrm{d}}$ \\
\hline G $108-36$ & $\mathrm{CC} 1$ & 2011 Jan 27 & 2011.0719 & HiCIAO & $K_{S}$ & $9.73 \pm 0.52$ & $7.962 \pm 0.031$ & $0.56 \pm 0.20$ & $\ldots$ & $\ldots$ & 176.8 & \\
\hline G $108-36$ & $\mathrm{CC} 1$ & 2012 Jan 3 & 2012.0066 & HiCIAO & $K_{S}$ & $9.51 \pm 0.35$ & $8.017 \pm 0.032$ & $1.84 \pm 0.20$ & $8.009 \pm 0.033$ & $1.86 \pm 0.21$ & 178.0 & BG \\
\hline G $108-36$ & $\mathrm{CC} 2$ & 2011 Jan 27 & 2011.0719 & HiCIAO & $K_{S}$ & $9.47 \pm 0.40$ & $8.246 \pm 0.032$ & $359.33 \pm 0.20$ & $\ldots$ & $\ldots$ & 183.1 & \\
\hline G $108-36$ & $\mathrm{CC} 2$ & 2012 Jan 3 & 2012.0066 & HiCIAO & $K_{S}$ & $9.20 \pm 0.25$ & $8.301 \pm 0.032$ & $0.62 \pm 0.19$ & $8.292 \pm 0.034$ & $0.58 \pm 0.20$ & 184.3 & BG \\
\hline G $108-36$ & $\mathrm{CC} 3$ & 2011 Jan 27 & 2011.0719 & HiCIAO & $K_{S}$ & $8.39 \pm 0.18$ & $5.307 \pm 0.025$ & $331.19 \pm 0.25$ & & & 117.8 & \\
\hline G $108-36$ & $\mathrm{CC} 3$ & 2012 Jan 3 & 2012.0066 & HiCIAO & $K_{S}$ & $8.16 \pm 0.09$ & $5.274 \pm 0.025$ & $333.21 \pm 0.25$ & $5.263 \pm 0.028$ & $333.15 \pm 0.27$ & 117.1 & BG \\
\hline G $108-36$ & $\mathrm{CC} 4$ & 2011 Jan 27 & 2011.0719 & HiCIAO & $K_{S}$ & $10.69 \pm 1.05$ & $8.603 \pm 0.033$ & $281.31 \pm 0.19$ & $\ldots$ & $\ldots$ & 191.0 & $\ldots$ \\
\hline G 108-36 & $\mathrm{CC} 4$ & 2012 Jan 3 & 2012.0066 & HiCIAO & $K_{S}$ & $10.50 \pm 0.94$ & $8.458 \pm 0.033$ & $282.07 \pm 0.19$ & $8.438 \pm 0.033$ & $281.86 \pm 0.20$ & 187.8 & BG \\
\hline G $108-36$ & $\mathrm{CC} 5$ & 2011 Jan 27 & 2011.0719 & HiCIAO & $K_{S}$ & $8.97 \pm 0.28$ & $9.317 \pm 0.035$ & $262.90 \pm 0.18$ & & & 206.8 & $B G^{d}$ \\
\hline G $108-36$ & $\mathrm{CC} 5$ & 2012 Jan 3 & 2012.0066 & HiCIAO & $K_{S}$ & $8.83 \pm 0.21$ & $9.152 \pm 0.034$ & $263.25 \pm 0.19$ & $9.135 \pm 0.035$ & $263.06 \pm 0.19$ & 203.2 & $\mathrm{BG}$ \\
\hline G $108-36$ & CC6 & 2011 Jan 27 & 2011.0719 & HiCIAO & $K_{S}$ & $9.14 \pm 0.71$ & $12.358 \pm 0.043$ & $178.39 \pm 0.17$ & $\ldots$ & $\ldots$ & 274.3 & $\mathrm{BG}^{\mathrm{d}}$ \\
\hline G $108-36$ & $\mathrm{CC} 7$ & 2012 Jan 3 & 2012.0066 & HiCIAO & $K_{S}$ & $10.98 \pm 1.13$ & $8.376 \pm 0.032$ & $20.57 \pm 0.19$ & $\ldots$ & $\ldots$ & 185.9 & SE \\
\hline G $108-36$ & $\mathrm{CC} 8$ & 2012 Jan 3 & 2012.0066 & HiCIAO & $K_{S}$ & $7.07 \pm 0.07$ & $11.346 \pm 0.040$ & $233.06 \pm 0.17$ & $\ldots$ & $\ldots$ & 251.9 & SE \\
\hline 1RXS J073829.3+240014 & $\mathrm{CC} 1$ & 2010 Nov 27 & 2010.9052 & $\mathrm{NIRC} 2 / \mathrm{N}$ & $H$ & $13.47 \pm 0.28$ & $5.481 \pm 0.003$ & $227.50 \pm 0.20$ & $\ldots$ & $\ldots$ & 103.6 & $\ldots$ \\
\hline 1RXS J073829.3+240014 & $\mathrm{CC} 1$ & 2013 Jan 17 & 2013.0448 & $\mathrm{NIRC} 2 / \mathrm{N}$ & $K$ & $13.44 \pm 1.24$ & $5.061 \pm 0.003$ & $226.11 \pm 0.20$ & $5.020 \pm 0.017$ & $226.07 \pm 0.30$ & 95.7 & BG \\
\hline GJ $3577 \mathrm{~A}$ & $\mathrm{CC} 1$ & $2013 \mathrm{Feb} 4$ & 2013.0944 & $\mathrm{NIRC} 2 / \mathrm{N}$ & $K_{S}$ & $13.59 \pm 1.04$ & $2.634 \pm 0.003$ & $311.27 \pm 0.20$ & $\ldots$ & $\ldots$ & 65.6 & SE \\
\hline GJ $3577 \mathrm{~A}$ & $\mathrm{CC} 2$ & 2013 Feb 4 & 2013.0944 & $\mathrm{NIRC} 2 / \mathrm{N}$ & $K_{S}$ & $12.22 \pm 0.49$ & $4.541 \pm 0.003$ & $34.21 \pm 0.20$ & $\ldots$ & $\ldots$ & 113.1 & SE \\
\hline GJ $3578 \mathrm{~B}$ & $\mathrm{CC} 1$ & 2013 Feb 4 & 2013.0942 & $\mathrm{NIRC} 2 / \mathrm{N}$ & $K_{S}$ & $13.57 \pm 1.00$ & $3.573 \pm 0.003$ & $129.33 \pm 0.20$ & $\ldots$ & $\ldots$ & 89.0 & SE \\
\hline NLTT 26359 & $\mathrm{CC} 1$ & $2011 \operatorname{Dec} 27$ & 2011.9872 & HiCIAO & $K_{S}$ & $11.98 \pm 0.77$ & $9.617 \pm 0.036$ & $40.93 \pm 0.18$ & $\ldots$ & $\ldots$ & 179.8 & $\ldots$ \\
\hline NLTT 26359 & $\mathrm{CC} 1$ & 2013 May 2 & 2013.3323 & $\mathrm{NIRC} 2 / \mathrm{W}$ & $K_{S}$ & $\ldots c$ & $9.907 \pm 0.075$ & $42.79 \pm 0.20$ & $9.916 \pm 0.037$ & $42.57 \pm 0.18$ & 185.3 & $\ddot{\mathrm{BG}}$ \\
\hline NLTT 26359 & $\mathrm{CC} 2$ & 2011 Dec 27 & 2011.9872 & HiCIAO & $K_{S}$ & $12.39 \pm 0.97$ & $9.084 \pm 0.034$ & $107.07 \pm 0.19$ & & & 169.9 & \\
\hline NLTT 26359 & $\mathrm{CC} 2$ & 2013 May 2 & 2013.3323 & $\mathrm{NIRC} 2 / \mathrm{W}$ & $K_{S}$ & $\ldots c$ & $9.448 \pm 0.075$ & $106.32 \pm 0.20$ & $9.463 \pm 0.036$ & $106.12 \pm 0.17$ & 176.7 & $\mathrm{BG}$ \\
\hline NLTT 26359 & $\mathrm{CC} 3$ & 2013 May 2 & 2013.3323 & $\mathrm{NIRC} 2 / \mathrm{W}$ & $K_{S}$ & $\ldots c$ & $14.789 \pm 0.075$ & $37.22 \pm 0.20$ & $\ldots$ & $\ldots$ & 276.5 & SE \\
\hline NLTT 26359 & $\mathrm{CC} 4$ & 2013 May 2 & 2013.3323 & $\mathrm{NIRC} 2 / \mathrm{W}$ & $K_{S}$ & $\ldots c$ & $19.331 \pm 0.076$ & $133.89 \pm 0.20$ & $\ldots$ & $\ldots$ & 361.5 & SE \\
\hline NLTT 26359 & CC5 & 2013 May 2 & 2013.3323 & $\mathrm{NIRC} 2 / \mathrm{W}$ & $K_{S}$ & $\ldots c$ & $12.568 \pm 0.075$ & $275.07 \pm 0.20$ & $\ldots$ & $\ldots$ & 235.0 & SE \\
\hline NLTT 26359 & CC6 & 2013 May 2 & 2013.3323 & $\mathrm{NIRC} 2 / \mathrm{W}$ & $K_{S}$ & $\ldots \mathrm{c}$ & $15.287 \pm 0.076$ & $344.78 \pm 0.20$ & $\ldots$ & $\ldots$ & 285.9 & SE \\
\hline G $13-33 \mathrm{AB}$ & $\mathrm{CC} 1$ & 2011 Dec 28 & 2011.9901 & HiCIAO & $K_{S}$ & $4.70 \pm 0.47$ & $6.094 \pm 0.027$ & $224.03 \pm 0.23$ & & $\ldots$ & 91.4 & $\mathrm{BG}^{\mathrm{d}}$ \\
\hline G $13-33 \mathrm{AB}$ & $\mathrm{CC} 1$ & $2013 \mathrm{Feb} 4$ & 2013.0947 & $\mathrm{NIRC} 2 / \mathrm{N}$ & $K_{S}$ & $\ldots c$ & $5.776 \pm 0.003$ & $223.06 \pm 0.20$ & $5.842 \pm 0.028$ & $222.41 \pm 0.26$ & 86.6 & $\mathrm{BG}$ \\
\hline G $13-33 \mathrm{AB}$ & $\mathrm{CC} 2$ & 2012 May 21 & 2012.3870 & $\mathrm{NIRC} 2 / \mathrm{N}$ & $K_{S}$ & $12.22 \pm 0.68$ & $4.498 \pm 0.003$ & $166.19 \pm 0.20$ & & & 67.5 & $\ldots$ \\
\hline G $13-33 \mathrm{AB}$ & $\mathrm{CC} 2$ & $2013 \mathrm{Feb} 4$ & 2013.0947 & NIRC2/N & $K_{S}$ & $11.53 \pm 0.41$ & $4.424 \pm 0.003$ & $163.66 \pm 0.20$ & $4.433 \pm 0.015$ & $164.86 \pm 0.32$ & 66.4 & BG \\
\hline GJ $3729 \mathrm{AB}$ & $\mathrm{CC} 1$ & 2011 Jan 26 & 2011.0698 & HiCIAO & $K_{S}$ & $10.95 \pm 1.06$ & $9.384 \pm 0.035$ & $110.53 \pm 0.18$ & $\ldots$ & $\ldots$ & 159.5 & $\mathrm{BG}^{\mathrm{d}}$ \\
\hline GJ $3729 \mathrm{AB}$ & $\mathrm{CC} 1$ & 2012 Jan 3 & 2012.0072 & HiCIAO & $H$ & $10.98 \pm 0.20$ & $9.471 \pm 0.022$ & $109.32 \pm 0.18$ & $9.474 \pm 0.037$ & $108.88 \pm 0.19$ & 161.0 & BG \\
\hline GJ $3729 \mathrm{AB}$ & $\mathrm{CC} 2$ & 2011 Jun 21 & 2011.4689 & NIRC2/N & $H$ & $13.04 \pm 0.28$ & $1.627 \pm 0.003$ & $47.93 \pm 0.20$ & & & 27.7 & \\
\hline GJ $3729 \mathrm{AB}$ & $\mathrm{CC} 2$ & 2012 Jan 3 & 2012.0072 & HiCIAO & $H$ & $12.61 \pm 0.97$ & $1.765 \pm 0.020$ & $45.68 \pm 0.65$ & $1.734 \pm 0.015$ & $44.06 \pm 1.06$ & 30.0 & BG \\
\hline
\end{tabular}




\begin{tabular}{|c|c|c|c|c|c|c|c|c|c|c|c|c|}
\hline \multirow[b]{2}{*}{ Name } & \multirow[b]{2}{*}{ Cand. } & \multirow[b]{2}{*}{$\begin{array}{l}\text { UT Date } \\
\text { (Y-M-D) }\end{array}$} & \multirow[b]{2}{*}{ Epoch } & \multirow[b]{2}{*}{ Instrument $^{\mathrm{a}}$} & \multirow[b]{2}{*}{ Filt. } & \multirow[b]{2}{*}{$\begin{array}{c}\text { Contrast } \\
\text { (mag) }\end{array}$} & \multicolumn{2}{|c|}{ Measured Position } & \multicolumn{2}{|c|}{ Background Position } & \multirow[b]{2}{*}{$\begin{array}{l}\text { Proj. Sep. } \\
\text { (AU) }\end{array}$} & \multirow[b]{2}{*}{ Comp.? } \\
\hline & & & & & & & $\begin{array}{c}\rho \\
\left({ }^{\prime \prime}\right)\end{array}$ & $\begin{array}{c}\text { P.A. } \\
\left({ }^{\circ}\right)\end{array}$ & $\begin{array}{c}\rho \\
\left({ }^{\prime \prime}\right)\end{array}$ & $\begin{array}{c}\text { P.A. } \\
\left({ }^{\circ}\right)\end{array}$ & & \\
\hline GJ $3729 \mathrm{AB}$ & $\mathrm{CC} 3$ & 2012 Jan 3 & 2012.0072 & HiCIAO & $H$ & $12.60 \pm 0.98$ & $9.489 \pm 0.022$ & $7.76 \pm 0.18$ & $\ldots$ & $\ldots$ & 161.3 & SE \\
\hline GJ $3729 \mathrm{AB}$ & $\mathrm{CC} 4$ & 2012 Jan 3 & 2012.0072 & HiCIAO & $H$ & $11.79 \pm 0.80$ & $11.195 \pm 0.023$ & $252.88 \pm 0.17$ & $\ldots$ & $\ldots$ & 190.3 & SE \\
\hline 1RXS J124147.5+564506 & $\mathrm{CC} 1$ & 2012 May 10 & 2012.3568 & HiCIAO & $K_{S}$ & $10.31 \pm 0.92$ & $8.995 \pm 0.034$ & $7.56 \pm 0.19$ & $\ldots$ & $\ldots$ & 269.9 & \\
\hline 1RXS J124147.5+564506 & $\mathrm{CC} 1$ & 2013 May 2 & 2013.3323 & $\mathrm{NIRC} 2 / \mathrm{W}$ & $K_{S}$ & $\ldots c$ & $9.042 \pm 0.075$ & $7.55 \pm 0.20$ & $8.978 \pm 0.035$ & $6.85 \pm 0.19$ & 271.3 & AMB \\
\hline 1RXS J124147.5+564506 & $\mathrm{CC} 2$ & 2013 May 2 & 2013.3323 & $\mathrm{NIRC} 2 / \mathrm{W}$ & $K_{S}$ & $\ldots{ }^{c}$ & $12.437 \pm 0.075$ & $238.98 \pm 0.20$ & $\ldots$ & $\ldots$ & 373.1 & SE \\
\hline 1RXS J124147.5+564506 & $\mathrm{CC} 3$ & 2013 May 2 & 2013.3323 & $\mathrm{NIRC} 2 / \mathrm{W}$ & $K_{S}$ & $\ldots$ c & $22.692 \pm 0.077$ & $240.72 \pm 0.20$ & $\ldots$ & $\ldots$ & 680.8 & $\mathrm{BG}^{\mathrm{d}}$ \\
\hline LHS 2613 & $\mathrm{CC} 1$ & 2012 Jan 3 & 2012.0071 & HiCIAO & $H$ & $9.97 \pm 0.09$ & $11.596 \pm 0.024$ & $23.13 \pm 0.17$ & $\ldots$ & $\ldots$ & 122.9 & $\mathrm{BG}^{\mathrm{d}, \mathrm{e}}$ \\
\hline 2MASS J14124864-1629561 & $\mathrm{CC} 1$ & 2012 May 22 & 2012.3899 & $\mathrm{NIRC} 2 / \mathrm{N}$ & $K_{S}$ & $11.92 \pm 0.39$ & $2.774 \pm 0.003$ & $233.63 \pm 0.20$ & $\ldots$ & $\ldots$ & 74.9 & $\ldots$ \\
\hline 2MASS J14124864-1629561 & $\mathrm{CC} 1$ & 2013 May 2 & 2013.3324 & $\mathrm{NIRC} 2 / \mathrm{N}$ & $K_{S}$ & $11.87 \pm 0.25$ & $2.645 \pm 0.003$ & $233.86 \pm 0.20$ & $2.644 \pm 0.004$ & $234.08 \pm 0.22$ & 71.4 & BG \\
\hline 2MASS J14124864-1629561 & $\mathrm{CC} 2$ & 2012 May 22 & 2012.3899 & $\mathrm{NIRC} 2 / \mathrm{N}$ & $K_{S}$ & $12.41 \pm 0.92$ & $6.656 \pm 0.003$ & $147.53 \pm 0.20$ & $\ldots$ & $\ldots$ & 179.7 & SE \\
\hline 2MASS J14124864-1629561 & $\mathrm{CC} 3$ & 2013 May 2 & 2013.3324 & $\mathrm{NIRC} 2 / \mathrm{N}$ & $K_{S}$ & $13.39 \pm 0.93$ & $5.018 \pm 0.003$ & $131.30 \pm 0.20$ & $\ldots$ & $\ldots$ & 135.5 & SE \\
\hline G $166-49$ & $\mathrm{CC} 1$ & 2012 May 22 & 2012.3896 & $\mathrm{NIRC} 2 / \mathrm{N}$ & $H$ & $14.01 \pm 0.46$ & $2.481 \pm 0.003$ & $265.28 \pm 0.20$ & $\ldots$ & $\ldots$ & 31.0 & $\ldots$ \\
\hline G 166-49 & $\mathrm{CC} 1$ & 2013 Feb 4 & 2013.0948 & $\mathrm{NIRC} 2 / \mathrm{N}$ & $K_{S}$ & $13.15 \pm 0.43$ & $2.326 \pm 0.003$ & $269.62 \pm 0.20$ & $2.325 \pm 0.008$ & $270.03 \pm 0.26$ & 29.1 & BG \\
\hline G 166-49 & $\mathrm{CC} 2$ & 2012 May 22 & 2012.3896 & $\mathrm{NIRC} 2 / \mathrm{N}$ & $H$ & $13.50 \pm 0.45$ & $4.455 \pm 0.003$ & $226.01 \pm 0.20$ & $\ldots$ & $\ldots$ & 55.7 & $\ldots$ \\
\hline G 166-49 & $\mathrm{CC} 2$ & 2013 Feb 4 & 2013.0948 & $\mathrm{NIRC} 2 / \mathrm{N}$ & $K_{S}$ & $12.84 \pm 0.68$ & $4.207 \pm 0.003$ & $226.38 \pm 0.20$ & $4.206 \pm 0.006$ & $226.63 \pm 0.23$ & 52.6 & BG \\
\hline G 166-49 & $\mathrm{CC} 3$ & 2012 May 22 & 2012.3896 & $\mathrm{NIRC} 2 / \mathrm{N}$ & $H$ & $6.09 \pm 0.31$ & $3.809 \pm 0.003$ & $54.11 \pm 0.20$ & $\ldots$ & $\ldots$ & 47.6 & $\mathrm{BG}^{\mathrm{d}}$ \\
\hline G $166-49$ & $\mathrm{CC} 3$ & $2013 \mathrm{Feb} 4$ & 2013.0948 & $\mathrm{NIRC} 2 / \mathrm{N}$ & $K_{S}$ & $\ldots{ }^{\mathrm{c}}$ & $4.035 \pm 0.003$ & $52.93 \pm 0.20$ & $4.049 \pm 0.007$ & $52.96 \pm 0.22$ & 50.4 & BG \\
\hline 2MASS J15323737+4653048 & $\mathrm{CC} 1$ & 2012 May 22 & 2012.3901 & $\mathrm{NIRC} 2 / \mathrm{N}$ & $K_{S}$ & $13.16 \pm 0.99$ & $2.658 \pm 0.003$ & $208.35 \pm 0.20$ & $\ldots$ & $\ldots$ & 87.7 & $\ldots$ \\
\hline 2MASS J15323737+4653048 & $\mathrm{CC} 1$ & 2013 Aug 17 & 2013.6249 & NIRC2/N & $K_{S}$ & $13.06 \pm 0.23$ & $2.515 \pm 0.003$ & $205.17 \pm 0.20$ & $2.547 \pm 0.015$ & $205.23 \pm 0.25$ & 83.0 & BG \\
\hline G $202-48$ & $\mathrm{CC} 1$ & 2011 Jun 21 & 2011.4691 & $\mathrm{NIRC} 2 / \mathrm{N}$ & $H$ & $15.91 \pm 0.89$ & $5.426 \pm 0.003$ & $201.15 \pm 0.20$ & $\ldots$ & $\ldots$ & 35.4 & SE \\
\hline GJ $3966 \mathrm{AB}$ & $\mathrm{CC} 1$ & 2011 Jun 21 & 2011.4692 & $\mathrm{NIRC} 2 / \mathrm{N}$ & $H$ & $12.19 \pm 0.23$ & $2.246 \pm 0.003$ & $202.44 \pm 0.20$ & $\ldots$ & $\ldots$ & 36.4 & $\ldots$ \\
\hline GJ $3966 \mathrm{AB}$ & $\mathrm{CC} 1$ & 2012 Apr 12 & 2012.2810 & $\mathrm{NIRC} 2 / \mathrm{N}$ & $H$ & $12.04 \pm 1.22$ & $2.235 \pm 0.003$ & $207.39 \pm 0.20$ & $2.201 \pm 0.007$ & $208.15 \pm 0.28$ & 36.2 & BG \\
\hline GJ $669 \mathrm{~A}$ & $\mathrm{CC} 1$ & 2012 May 10 & 2012.3574 & HiCIAO & $H$ & $13.62 \pm 0.76$ & $3.694 \pm 0.020$ & $297.88 \pm 0.33$ & $\ldots$ & 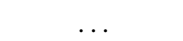 & 43.2 & \\
\hline GJ 669 A & $\mathrm{CC} 1$ & 2013 May 2 & 2013.3329 & NIRC2/W & $K_{S}$ & $\ldots c$ & $3.233 \pm 0.074$ & $293.43 \pm 0.20$ & $3.370 \pm 0.020$ & $294.29 \pm 0.36$ & 37.8 & BG \\
\hline GJ 669 A & $\mathrm{CC} 2$ & 2012 May 10 & 2012.3574 & HiCIAO & $H$ & $13.64 \pm 0.79$ & $8.801 \pm 0.022$ & $22.80 \pm 0.19$ & $\ldots$ & 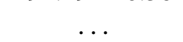 & 103.0 & $\ldots$ \\
\hline GJ $669 \mathrm{~A}$ & $\mathrm{CC} 2$ & 2013 May 2 & 2013.3329 & NIRC $2 / \mathrm{W}$ & $K_{S}$ & $\ldots c$ & $8.584 \pm 0.075$ & $25.28 \pm 0.20$ & $8.568 \pm 0.023$ & $24.88 \pm 0.20$ & 100.4 & $\mathrm{BG}$ \\
\hline GJ $669 \mathrm{~A}$ & $\mathrm{CC} 3$ & 2012 May 10 & 2012.3574 & HiCIAO & $H$ & $9.10 \pm 0.08$ & $10.380 \pm 0.023$ & $156.61 \pm 0.18$ & $\ldots$ & $\ldots$ & 121.4 & $\ldots$ \\
\hline GJ $669 \mathrm{~A}$ & $\mathrm{CC} 3$ & 2013 May 2 & 2013.3329 & NIRC2/W & $K_{S}$ & $\ldots$ c & $10.753 \pm 0.075$ & $157.09 \pm 0.20$ & $10.769 \pm 0.023$ & $156.39 \pm 0.17$ & 125.8 & $\mathrm{BG}$ \\
\hline GJ 669 A & $\mathrm{CC} 4$ & 2012 May 10 & 2012.3574 & HiCIAO & $H$ & $9.68 \pm 0.08$ & $10.538 \pm 0.023$ & $160.77 \pm 0.18$ & $20=-800$ & 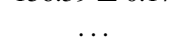 & 123.3 & $\ldots$ \\
\hline GJ $669 \mathrm{~A}$ & $\mathrm{CC} 4$ & 2013 May 2 & 2013.3329 & NIRC2/W & $K_{S}$ & $\ldots c$ & $10.916 \pm 0.075$ & $161.16 \pm 0.20$ & $10.924 \pm 0.022$ & $160.39 \pm 0.17$ & 127.7 & BG \\
\hline GJ 669 A & $\mathrm{CC} 5$ & 2012 May 10 & 2012.3574 & HiCIAO & $H$ & $12.99 \pm 0.41$ & $8.960 \pm 0.022$ & $200.63 \pm 0.19$ & $\ldots$ & $\ldots$ & 104.8 & $\ldots$ \\
\hline GJ 669 A & $\mathrm{CC} 5$ & 2013 May 2 & 2013.3329 & $\mathrm{NIRC} 2 / \mathrm{W}$ & $K_{S}$ & $\ldots c$ & $9.270 \pm 0.075$ & $199.43 \pm 0.20$ & $9.215 \pm 0.023$ & $198.76 \pm 0.19$ & 108.5 & BG \\
\hline GJ 669 A & CC6 & 2013 May 2 & 2013.3329 & NIRC $2 / \mathrm{W}$ & $K_{S}$ & $\ldots \mathrm{c}$ & $11.173 \pm 0.075$ & $91.22 \pm 0.20$ & $\ldots$ & $\ldots$ & 130.7 & SE \\
\hline LHS 3321 & $\mathrm{CC} 1$ & 2011 Jun 21 & 2011.4692 & NIRC2/N & $H$ & $13.58 \pm 0.18$ & $2.615 \pm 0.003$ & $279.74 \pm 0.20$ & 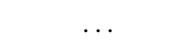 & $\ldots$ & 24.8 & \\
\hline LHS 3321 & $\mathrm{CC} 1$ & 2012 May 10 & 2012.3572 & HiCIAO & $H$ & $13.06 \pm 0.27$ & $2.812 \pm 0.020$ & $289.50 \pm 0.42$ & $2.835 \pm 0.008$ & $290.63 \pm 0.23$ & 26.7 & BG \\
\hline LHS 3321 & $\mathrm{CC} 2$ & 2012 May 10 & 2012.3572 & HiCIAO & $H$ & $12.12 \pm 0.11$ & $9.222 \pm 0.022$ & $314.35 \pm 0.19$ & $\ldots$ & $\ldots$ & 87.4 & SE \\
\hline LP 390-16 & $\mathrm{CC} 1$ & 2011 Mar 25 & 2011.2289 & $\mathrm{NIRC} 2 / \mathrm{N}$ & $K_{S}$ & $7.35 \pm 0.36$ & $4.086 \pm 0.003$ & $262.30 \pm 0.20$ & $\ldots$ & $\ldots$ & 70.3 & $\mathrm{BG}^{\mathrm{d}}$ \\
\hline GJ $9652 \mathrm{~A}$ & $\mathrm{CC} 1$ & 2011 Aug 20 & 2011.6334 & $\mathrm{NIRC} 2 / \mathrm{N}$ & $H$ & $\ldots \mathrm{c}$ & $2.770 \pm 0.003$ & $341.09 \pm 0.20$ & $\ldots$ & $\ldots$ & 52.9 & $\mathrm{BG}^{\mathrm{d}}$ \\
\hline GJ $9652 \mathrm{~A}$ & $\mathrm{CC} 1$ & 2012 Aug 23 & 2012.6442 & $\mathrm{NIRC} 2 / \mathrm{N}$ & $H$ & $\ldots{ }^{c}$ & $2.201 \pm 0.003$ & $353.00 \pm 0.20$ & $2.199 \pm 0.009$ & $352.92 \pm 0.33$ & 42.0 & BG \\
\hline GJ $9652 \mathrm{~A}$ & $\mathrm{CC} 2$ & 2011 Aug 20 & 2011.6334 & $\mathrm{NIRC} 2 / \mathrm{N}$ & $H$ & $\ldots{ }^{c}$ & $2.844 \pm 0.003$ & $10.26 \pm 0.20$ & $\ldots$ & $\ldots$ & 54.3 & $\ldots$ \\
\hline GJ $9652 \mathrm{~A}$ & $\mathrm{CC} 2$ & 2012 Aug 23 & 2012.6442 & $\mathrm{NIRC} 2 / \mathrm{N}$ & $H$ & $\ldots \mathrm{c}$ & $2.619 \pm 0.003$ & $25.62 \pm 0.20$ & $2.619 \pm 0.009$ & $25.64 \pm 0.28$ & 50.0 & BG \\
\hline GJ $9652 \mathrm{~A}$ & $\mathrm{CC} 3$ & 2011 Aug 20 & 2011.6334 & NIRC2/N & $H$ & $12.45 \pm 0.99$ & $3.302 \pm 0.003$ & $44.01 \pm 0.20$ & $\ldots$ & $\ldots$ & 63.1 & $\ldots$ \\
\hline GJ 9652 A & $\mathrm{CC} 3$ & 2012 Aug 23 & 2012.6442 & $\mathrm{NIRC} 2 / \mathrm{N}$ & $H$ & $12.77 \pm 0.20$ & $3.503 \pm 0.003$ & $56.39 \pm 0.20$ & $3.504 \pm 0.009$ & $56.44 \pm 0.23$ & 66.9 & BG \\
\hline GJ $9652 \mathrm{~A}$ & $\mathrm{CC} 4$ & 2011 Aug 20 & 2011.6334 & $\mathrm{NIRC} 2 / \mathrm{N}$ & $H$ & $13.60 \pm 1.26$ & $4.048 \pm 0.003$ & $52.10 \pm 0.20$ & $\ldots$ & $\ldots$ & 77.3 & $\ldots$ \\
\hline GJ $9652 \mathrm{~A}$ & $\mathrm{CC} 4$ & 2012 Aug 23 & 2012.6442 & $\mathrm{NIRC} 2 / \mathrm{N}$ & $H$ & $13.80 \pm 1.17$ & $4.327 \pm 0.003$ & $61.63 \pm 0.20$ & $4.335 \pm 0.009$ & $61.80 \pm 0.22$ & 82.6 & BG \\
\hline
\end{tabular}


Table 8

(Continued)

\begin{tabular}{|c|c|c|c|c|c|c|c|c|c|c|c|c|}
\hline \multirow[b]{2}{*}{ Name } & \multirow[b]{2}{*}{ Cand. } & \multirow[b]{2}{*}{$\begin{array}{l}\text { UT Date } \\
\text { (Y-M-D) }\end{array}$} & \multirow[b]{2}{*}{ Epoch } & \multirow[b]{2}{*}{ Instrument $^{\mathrm{a}}$} & \multirow[b]{2}{*}{ Filt. } & \multirow[b]{2}{*}{$\begin{array}{c}\text { Contrast } \\
\text { (mag) }\end{array}$} & \multicolumn{2}{|c|}{ Measured Position } & \multicolumn{2}{|c|}{ Background Position } & \multirow[b]{2}{*}{$\begin{array}{l}\text { Proj. Sep. } \\
\text { (AU) }\end{array}$} & \multirow[b]{2}{*}{ Comp.? ${ }^{\mathrm{b}}$} \\
\hline & & & & & & & $\begin{array}{c}\rho \\
\left({ }^{\prime \prime}\right)\end{array}$ & $\begin{array}{c}\text { P.A. } \\
\left({ }^{\circ}\right)\end{array}$ & $\begin{array}{c}\rho \\
\left({ }^{\prime \prime}\right)\end{array}$ & $\begin{array}{c}\text { P.A. } \\
\left({ }^{\circ}\right)\end{array}$ & & \\
\hline GJ $9652 \mathrm{~A}$ & $\mathrm{CC} 5$ & 2011 Aug 20 & 2011.6334 & $\mathrm{NIRC} 2 / \mathrm{N}$ & $H$ & $12.87 \pm 1.14$ & $3.290 \pm 0.003$ & $61.39 \pm 0.20$ & & & 62.8 & \\
\hline GJ 9652 A & $\mathrm{CC} 5$ & 2012 Aug 23 & 2012.6442 & $\mathrm{NIRC} 2 / \mathrm{N}$ & $H$ & $14.12 \pm 0.97$ & $3.690 \pm 0.003$ & $71.99 \pm 0.20$ & $3.694 \pm 0.009$ & $72.06 \pm 0.22$ & 70.5 & BG \\
\hline GJ 9652 A & CC6 & 2011 Aug 20 & 2011.6334 & $\mathrm{NIRC} 2 / \mathrm{N}$ & $H$ & $13.68 \pm 1.26$ & $4.122 \pm 0.003$ & $64.75 \pm 0.20$ & $\ldots$ & $\ldots$ & 78.7 & $\ldots$ \\
\hline GJ $9652 \mathrm{~A}$ & CC6 & 2012 Aug 23 & 2012.6442 & $\mathrm{NIRC} 2 / \mathrm{N}$ & $H$ & $14.49 \pm 1.19$ & $4.553 \pm 0.003$ & $73.13 \pm 0.20$ & $4.550 \pm 0.009$ & $73.12 \pm 0.21$ & 87.0 & BG \\
\hline GJ 9652 A & $\mathrm{CC} 7$ & 2011 Aug 20 & 2011.6334 & $\mathrm{NIRC} 2 / \mathrm{N}$ & $H$ & $14.07 \pm 1.22$ & $5.043 \pm 0.003$ & $60.39 \pm 0.20$ & $\ldots$ & $\ldots$ & 96.3 & $\ldots$ \\
\hline GJ 9652 A & $\mathrm{CC} 7$ & 2012 Aug 23 & 2012.6442 & $\mathrm{NIRC} 2 / \mathrm{N}$ & $H$ & $13.61 \pm 0.73$ & $5.420 \pm 0.003$ & $67.35 \pm 0.20$ & $5.416 \pm 0.009$ & $67.71 \pm 0.20$ & 103.5 & BG \\
\hline GJ $9652 \mathrm{~A}$ & $\mathrm{CC} 8$ & 2011 Aug 20 & 2011.6334 & $\mathrm{NIRC} 2 / \mathrm{N}$ & $H$ & $14.92 \pm 1.33$ & $4.975 \pm 0.003$ & $63.07 \pm 0.20$ & $\ldots$ & $\ldots$ & 95.0 & SE \\
\hline GJ $9652 \mathrm{~A}$ & CC9 & 2011 Aug 20 & 2011.6334 & $\mathrm{NIRC} 2 / \mathrm{N}$ & $H$ & $12.27 \pm 0.32$ & $6.776 \pm 0.003$ & $48.88 \pm 0.20$ & $\ldots$ & $\ldots$ & 129.4 & SE \\
\hline GJ 9652 A & $\mathrm{CC} 10$ & 2011 Aug 20 & 2011.6334 & $\mathrm{NIRC} 2 / \mathrm{N}$ & $H$ & $7.64 \pm 0.28$ & $6.923 \pm 0.003$ & $51.68 \pm 0.20$ & $\ldots$ & $\ldots$ & 132.2 & $\mathrm{BG}^{\mathrm{d}}$ \\
\hline GJ $9652 \mathrm{~A}$ & $\mathrm{CC} 10$ & 2012 Aug 23 & 2012.6442 & $\mathrm{NIRC} 2 / \mathrm{N}$ & $H$ & $7.67 \pm 0.17$ & $7.177 \pm 0.003$ & $57.19 \pm 0.20$ & $7.180 \pm 0.009$ & $57.53 \pm 0.21$ & 137.1 & BG \\
\hline GJ $9652 \mathrm{~A}$ & $\mathrm{CC} 11$ & 2011 Aug 20 & 2011.6334 & $\mathrm{NIRC} 2 / \mathrm{N}$ & $H$ & $11.64 \pm 0.39$ & $4.283 \pm 0.003$ & $90.74 \pm 0.20$ & $\ldots$ & $\ldots$ & 81.8 & SE \\
\hline GJ 9652 A & $\mathrm{CC} 12$ & 2011 Aug 20 & 2011.6334 & NIRC2/N & $H$ & $13.37 \pm 1.14$ & $4.650 \pm 0.003$ & $87.70 \pm 0.20$ & $\ldots$ & $\ldots$ & 88.8 & SE \\
\hline GJ 9652 A & $\mathrm{CC} 13$ & 2011 Aug 20 & 2011.6334 & NIRC2/N & $H$ & $12.32 \pm 1.13$ & $2.566 \pm 0.003$ & $95.62 \pm 0.20$ & $\ldots$ & $\ldots$ & 49.0 & $\ldots$ \\
\hline GJ 9652 A & $\mathrm{CC} 13$ & 2012 Aug 23 & 2012.6442 & NIRC2/N & $H$ & $13.91 \pm 0.32$ & $3.255 \pm 0.003$ & $102.11 \pm 0.20$ & $3.254 \pm 0.009$ & $102.24 \pm 0.21$ & 62.2 & BG \\
\hline GJ 9652 A & $\mathrm{CC} 14$ & 2011 Aug 20 & 2011.6334 & $\mathrm{NIRC} 2 / \mathrm{N}$ & $H$ & $13.91 \pm 1.06$ & $5.873 \pm 0.003$ & $128.27 \pm 0.20$ & $\ldots$ & $\ldots$ & 112.2 & SE \\
\hline GJ $9652 \mathrm{~A}$ & $\mathrm{CC} 15$ & 2011 Aug 20 & 2011.6334 & $\mathrm{NIRC} 2 / \mathrm{N}$ & $H$ & $14.30 \pm 1.12$ & $6.833 \pm 0.003$ & $134.76 \pm 0.20$ & $\ldots$ & $\ldots$ & 130.5 & SE \\
\hline GJ 9652 A & $\mathrm{CC} 16$ & 2011 Aug 20 & 2011.6334 & $\mathrm{NIRC} 2 / \mathrm{N}$ & $H$ & $10.86 \pm 0.28$ & $7.759 \pm 0.003$ & $134.48 \pm 0.20$ & $\ldots$ & $\ldots$ & 148.2 & SE \\
\hline GJ 9652 A & $\mathrm{CC} 17$ & 2011 Aug 20 & 2011.6334 & NIRC2/N & $H$ & $14.32 \pm 1.34$ & $4.752 \pm 0.003$ & $175.02 \pm 0.20$ & $\ldots$ & $\ldots$ & 90.8 & SE \\
\hline GJ 9652 A & $\mathrm{CC} 18$ & 2011 Aug 20 & 2011.6334 & $\mathrm{NIRC} 2 / \mathrm{N}$ & $H$ & $11.64 \pm 1.19$ & $1.766 \pm 0.003$ & $167.88 \pm 0.20$ & & & 33.7 & $\ldots$ \\
\hline GJ $9652 \mathrm{~A}$ & $\mathrm{CC} 18$ & 2012 Aug 23 & 2012.6442 & $\mathrm{NIRC} 2 / \mathrm{N}$ & $H$ & $12.59 \pm 0.20$ & $2.384 \pm 0.003$ & $155.25 \pm 0.20$ & $2.383 \pm 0.009$ & $155.26 \pm 0.24$ & 45.5 & BG \\
\hline GJ $9652 \mathrm{~A}$ & CC19 & 2011 Aug 20 & 2011.6334 & $\mathrm{NIRC} 2 / \mathrm{N}$ & $H$ & $10.63 \pm 0.76$ & $1.770 \pm 0.003$ & $174.79 \pm 0.20$ & 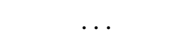 & $\ldots$ & 33.8 & $\ldots$ \\
\hline GJ $9652 \mathrm{~A}$ & CC19 & 2012 Aug 23 & 2012.6442 & NIRC2/N & $H$ & $10.74 \pm 0.17$ & $2.332 \pm 0.003$ & $160.20 \pm 0.20$ & $2.337 \pm 0.009$ & $160.30 \pm 0.24$ & 44.5 & BG \\
\hline GJ $9652 \mathrm{~A}$ & $\mathrm{CC} 20$ & 2011 Aug 20 & 2011.6334 & $\mathrm{NIRC} 2 / \mathrm{N}$ & $H$ & $11.62 \pm 0.59$ & $3.108 \pm 0.003$ & $255.69 \pm 0.20$ & $\ldots$ & $\ldots$ & 59.4 & $\ldots$ \\
\hline GJ $9652 \mathrm{~A}$ & $\mathrm{CC} 20$ & 2012 Aug 23 & 2012.6442 & NIRC2/N & $H$ & $11.43 \pm 0.17$ & $2.674 \pm 0.003$ & $243.29 \pm 0.20$ & $2.673 \pm 0.009$ & $243.17 \pm 0.29$ & 51.1 & BG \\
\hline GJ 9652 A & $\mathrm{CC} 21$ & 2011 Aug 20 & 2011.6334 & NIRC2/N & $H$ & $15.65 \pm 2.22$ & $4.879 \pm 0.003$ & $299.76 \pm 0.20$ & & & 93.2 & $\ldots$ \\
\hline GJ 9652 A & $\mathrm{CC} 21$ & 2012 Aug 23 & 2012.6442 & NIRC2/N & $H$ & $10.81 \pm 1.26$ & $4.118 \pm 0.003$ & $298.81 \pm 0.20$ & $4.118 \pm 0.009$ & $298.79 \pm 0.26$ & 78.7 & BG \\
\hline GJ $9652 \mathrm{~A}$ & $\mathrm{CC} 22$ & 2011 Aug 20 & 2011.6334 & $\mathrm{NIRC} 2 / \mathrm{N}$ & $H$ & $5.29 \pm 0.28$ & $4.868 \pm 0.003$ & $318.20 \pm 0.20$ & $\ldots$ & $\ldots$ & 93.0 & $\mathrm{BG}^{\mathrm{d}}$ \\
\hline GJ $9652 \mathrm{~A}$ & $\mathrm{CC} 22$ & 2012 Aug 23 & 2012.6442 & NIRC2/N & $H$ & $5.23 \pm 0.17$ & $4.129 \pm 0.003$ & $320.70 \pm 0.20$ & $4.127 \pm 0.009$ & $320.64 \pm 0.26$ & 78.9 & BG \\
\hline GJ $9652 \mathrm{~A}$ & $\mathrm{CC} 23$ & 2012 Aug 23 & 2012.6442 & $\mathrm{NIRC} 2 / \mathrm{N}$ & $H$ & $13.97 \pm 1.19$ & $3.931 \pm 0.003$ & $13.79 \pm 0.20$ & $\ldots$ & $\ldots$ & 75.1 & SE \\
\hline GJ 9652 A & $\mathrm{CC} 24$ & 2012 Aug 23 & 2012.6442 & $\mathrm{NIRC} 2 / \mathrm{N}$ & $H$ & $13.84 \pm 1.16$ & $4.390 \pm 0.003$ & $10.93 \pm 0.20$ & $\ldots$ & $\ldots$ & 83.8 & SE \\
\hline GJ $9652 \mathrm{~A}$ & $\mathrm{CC} 25$ & 2012 Aug 23 & 2012.6442 & $\mathrm{NIRC} 2 / \mathrm{N}$ & $H$ & $14.36 \pm 0.79$ & $5.068 \pm 0.003$ & $6.62 \pm 0.20$ & $\ldots$ & $\ldots$ & 96.8 & SE \\
\hline GJ $9652 \mathrm{~A}$ & CC26 & 2012 Aug 23 & 2012.6442 & $\mathrm{NIRC} 2 / \mathrm{N}$ & $H$ & $14.77 \pm 1.03$ & $5.471 \pm 0.003$ & $6.19 \pm 0.20$ & $\ldots$ & $\ldots$ & 104.5 & SE \\
\hline GJ 9652 A & $\mathrm{CC} 27$ & 2012 Aug 23 & 2012.6442 & $\mathrm{NIRC} 2 / \mathrm{N}$ & $H$ & $15.06 \pm 1.11$ & $5.117 \pm 0.003$ & $24.63 \pm 0.20$ & $\ldots$ & $\ldots$ & 97.7 & SE \\
\hline GJ 9652 A & $\mathrm{CC} 28$ & 2012 Aug 23 & 2012.6442 & $\mathrm{NIRC} 2 / \mathrm{N}$ & $H$ & $13.32 \pm 0.29$ & $5.102 \pm 0.003$ & $236.82 \pm 0.20$ & $\ldots$ & $\ldots$ & 97.5 & SE \\
\hline GJ $9652 \mathrm{~A}$ & $\mathrm{CC} 29$ & 2012 Aug 23 & 2012.6442 & $\mathrm{NIRC} 2 / \mathrm{N}$ & $H$ & $13.16 \pm 0.83$ & $5.354 \pm 0.003$ & $254.07 \pm 0.20$ & $\ldots$ & $\ldots$ & 102.3 & SE \\
\hline GJ $9652 \mathrm{~A}$ & CC30 & 2012 Aug 23 & 2012.6442 & $\mathrm{NIRC} 2 / \mathrm{N}$ & $H$ & $9.02 \pm 0.17$ & $5.360 \pm 0.003$ & $258.03 \pm 0.20$ & $\ldots$ & $\ldots$ & 102.4 & SE \\
\hline GJ $9652 \mathrm{~A}$ & CC 31 & 2012 Aug 23 & 2012.6442 & NIRC2/N & $H$ & $13.79 \pm 0.97$ & $6.927 \pm 0.003$ & $241.92 \pm 0.20$ & $\ldots$ & $\ldots$ & 132.3 & SE \\
\hline GJ 9652 A & $\mathrm{CC} 32$ & 2012 Aug 23 & 2012.6442 & $\mathrm{NIRC} 2 / \mathrm{N}$ & $H$ & $10.86 \pm 0.17$ & $5.766 \pm 0.003$ & $271.95 \pm 0.20$ & $\ldots$ & $\ldots$ & 110.1 & SE \\
\hline GJ 9652 A & CC33 & 2012 Aug 23 & 2012.6442 & $\mathrm{NIRC} 2 / \mathrm{N}$ & $H$ & $15.13 \pm 1.19$ & $5.460 \pm 0.003$ & $269.50 \pm 0.20$ & $\ldots$ & $\ldots$ & 104.3 & SE \\
\hline GJ 9652 A & $\mathrm{CC} 34$ & 2012 Aug 23 & 2012.6442 & $\mathrm{NIRC} 2 / \mathrm{N}$ & $H$ & $14.85 \pm 1.01$ & $5.153 \pm 0.003$ & $295.41 \pm 0.20$ & $\ldots$ & $\ldots$ & 98.4 & SE \\
\hline GJ $9652 \mathrm{~A}$ & CC35 & 2012 Aug 23 & 2012.6442 & NIRC2/N & $H$ & $13.11 \pm 0.27$ & $4.875 \pm 0.003$ & $299.09 \pm 0.20$ & $\ldots$ & $\ldots$ & 93.1 & SE \\
\hline GJ $9652 \mathrm{~A}$ & CC36 & 2012 Aug 23 & 2012.6442 & NIRC2/N & $H$ & $14.04 \pm 0.89$ & $6.482 \pm 0.003$ & $312.68 \pm 0.20$ & $\ldots$ & $\ldots$ & 123.8 & SE \\
\hline GJ 9652 A & $\mathrm{CC} 37$ & 2012 Aug 23 & 2012.6442 & NIRC2/N & $H$ & $10.19 \pm 0.17$ & $6.564 \pm 0.003$ & $319.50 \pm 0.20$ & $\ldots$ & $\ldots$ & 125.4 & SE \\
\hline GJ $9652 \mathrm{~A}$ & $\mathrm{CC} 38$ & 2012 Aug 23 & 2012.6442 & NIRC2/N & $H$ & $14.35 \pm 1.19$ & $6.994 \pm 0.003$ & $323.57 \pm 0.20$ & $\ldots$ & $\ldots$ & 133.6 & SE \\
\hline
\end{tabular}




\begin{tabular}{|c|c|c|c|c|c|c|c|c|c|c|c|c|}
\hline \multirow[b]{2}{*}{ Name } & \multirow[b]{2}{*}{ Cand. } & \multirow[b]{2}{*}{$\begin{array}{l}\text { UT Date } \\
\text { (Y-M-D) }\end{array}$} & \multirow[b]{2}{*}{ Epoch } & \multirow[b]{2}{*}{ Instrument $\mathrm{t}^{\mathrm{a}}$} & \multirow[b]{2}{*}{ Filt. } & \multirow[b]{2}{*}{$\begin{array}{c}\text { Contrast } \\
\text { (mag) }\end{array}$} & \multicolumn{2}{|c|}{ Measured Position } & \multicolumn{2}{|c|}{ Background Position } & \multirow[b]{2}{*}{$\begin{array}{l}\text { Proj. Sep. } \\
\text { (AU) }\end{array}$} & \multirow[b]{2}{*}{ Comp. $?^{\mathrm{b}}$} \\
\hline & & & & & & & $\begin{array}{c}\rho \\
\left({ }^{\prime \prime}\right)\end{array}$ & $\begin{array}{c}\text { P.A. } \\
\left({ }^{\circ}\right)\end{array}$ & $\begin{array}{c}\rho \\
\left({ }^{\prime \prime}\right)\end{array}$ & $\begin{array}{c}\text { P.A. } \\
\left({ }^{\circ}\right)\end{array}$ & & \\
\hline GJ $9652 \mathrm{~A}$ & CC39 & 2012 Aug 23 & 2012.6442 & $\mathrm{NIRC} 2 / \mathrm{N}$ & $H$ & $14.49 \pm 1.06$ & $6.465 \pm 0.003$ & $329.59 \pm 0.20$ & $\ldots$ & $\ldots$ & 123.5 & SE \\
\hline GJ $9652 \mathrm{~A}$ & $\mathrm{CC} 40$ & 2012 Aug 23 & 2012.6442 & $\mathrm{NIRC} 2 / \mathrm{N}$ & $H$ & $13.07 \pm 0.34$ & $7.556 \pm 0.003$ & $335.78 \pm 0.20$ & $\ldots$ & $\ldots$ & 144.3 & SE \\
\hline GJ $9652 \mathrm{~A}$ & $\mathrm{CC} 41$ & 2012 Aug 23 & 2012.6442 & $\mathrm{NIRC} 2 / \mathrm{N}$ & $H$ & $15.04 \pm 1.10$ & $5.578 \pm 0.003$ & $352.79 \pm 0.20$ & $\ldots$ & $\ldots$ & 106.5 & SE \\
\hline GJ $9652 \mathrm{~A}$ & $\mathrm{CC} 42$ & 2012 Aug 23 & 2012.6442 & $\mathrm{NIRC} 2 / \mathrm{N}$ & $H$ & $12.45 \pm 1.26$ & $4.023 \pm 0.003$ & $1.14 \pm 0.20$ & $\ldots$ & $\ldots$ & 76.8 & SE \\
\hline 2MASS J19303829-1335083 & $\mathrm{CC} 1$ & 2010 Aug 17 & 2010.6251 & $\mathrm{NIRC} 2 / \mathrm{N}$ & $H$ & $9.68 \pm 0.22$ & $7.214 \pm 0.003$ & $70.62 \pm 0.20$ & $\ldots$ & $\ldots$ & 199.8 & \\
\hline 2MASS J19303829-1335083 & $\mathrm{CC} 1$ & 2011 Jul 1 & 2011.4969 & $\mathrm{NIRC} 2 / \mathrm{N}$ & $H$ & $10.18 \pm 0.30$ & $6.942 \pm 0.003$ & $72.64 \pm 0.20$ & $6.949 \pm 0.010$ & $72.40 \pm 0.22$ & 192.3 & BG \\
\hline 2MASS J19303829-1335083 & $\mathrm{CC} 2$ & 2010 Aug 17 & 2010.6251 & $\mathrm{NIRC} 2 / \mathrm{N}$ & $H$ & $10.79 \pm 0.31$ & $4.481 \pm 0.003$ & $83.86 \pm 0.20$ & $\ldots$ & $\ldots$ & 124.1 & $\ldots$ \\
\hline 2MASS J19303829-1335083 & $\mathrm{CC} 2$ & $2011 \mathrm{Jul} 1$ & 2011.4969 & $\mathrm{NIRC} 2 / \mathrm{N}$ & $H$ & $11.19 \pm 0.37$ & $4.276 \pm 0.003$ & $87.91 \pm 0.20$ & $4.278 \pm 0.010$ & $87.51 \pm 0.24$ & 118.4 & BG \\
\hline 2MASS J19303829-1335083 & $\mathrm{CC} 3$ & 2010 Aug 17 & 2010.6251 & $\mathrm{NIRC} 2 / \mathrm{N}$ & $H$ & $5.85 \pm 0.16$ & $2.174 \pm 0.003$ & $177.62 \pm 0.20$ & & & 60.2 & \\
\hline 2MASS J19303829-1335083 & $\mathrm{CC} 3$ & $2011 \mathrm{Jul} 1$ & 2011.4969 & $\mathrm{NIRC} 2 / \mathrm{N}$ & $H$ & $6.34 \pm 0.18$ & $2.503 \pm 0.003$ & $182.17 \pm 0.20$ & $2.467 \pm 0.010$ & $182.11 \pm 0.28$ & 69.3 & BG \\
\hline 2MASS J19303829-1335083 & $\mathrm{CC} 4$ & 2010 Aug 17 & 2010.6251 & $\mathrm{NIRC} 2 / \mathrm{N}$ & $H$ & $9.98 \pm 0.19$ & $3.528 \pm 0.003$ & $229.57 \pm 0.20$ & & & 97.7 & \\
\hline 2MASS J19303829-1335083 & $\mathrm{CC} 4$ & 2011 Jul 1 & 2011.4969 & $\mathrm{NIRC} 2 / \mathrm{N}$ & $H$ & $10.46 \pm 0.23$ & $3.875 \pm 0.003$ & $227.84 \pm 0.20$ & $3.857 \pm 0.010$ & $228.00 \pm 0.23$ & 107.3 & BG \\
\hline 1RXS J193528.9+374605 & $\mathrm{CC} 1$ & 2010 Aug 17 & 2010.6249 & $\mathrm{NIRC} 2 / \mathrm{N}$ & $H$ & $14.28 \pm 0.36$ & $5.844 \pm 0.003$ & $343.10 \pm 0.20$ & & & 58.4 & \\
\hline 1RXS J193528.9+374605 & $\mathrm{CC} 1$ & 2011 Aug 20 & 2011.6333 & $\mathrm{NIRC} 2 / \mathrm{N}$ & $H$ & $14.36 \pm 0.26$ & $5.891 \pm 0.003$ & $344.86 \pm 0.20$ & $5.903 \pm 0.006$ & $344.68 \pm 0.21$ & 58.9 & BG \\
\hline 1RXS J193528.9+374605 & $\mathrm{CC} 2$ & 2010 Aug 17 & 2010.6249 & $\mathrm{NIRC} 2 / \mathrm{N}$ & $H$ & $8.73 \pm 0.30$ & $2.759 \pm 0.003$ & $87.94 \pm 0.20$ & $\ldots$ & $\ldots$ & 27.6 & $\ldots$ \\
\hline 1RXS J193528.9+374605 & $\mathrm{CC} 2$ & 2011 Aug 20 & 2011.6333 & $\mathrm{NIRC} 2 / \mathrm{N}$ & $H$ & $8.86 \pm 0.15$ & $2.903 \pm 0.003$ & $86.40 \pm 0.20$ & $2.904 \pm 0.007$ & $86.02 \pm 0.21$ & 29.0 & BG \\
\hline 1RXS J193528.9+374605 & $\mathrm{CC} 3$ & 2010 Aug 17 & 2010.6249 & $\mathrm{NIRC} 2 / \mathrm{N}$ & $H$ & $13.91 \pm 0.32$ & $3.902 \pm 0.003$ & $88.24 \pm 0.20$ & & & 39.0 & \\
\hline 1RXS J193528.9+374605 & $\mathrm{CC} 3$ & 2011 Aug 20 & 2011.6333 & $\mathrm{NIRC} 2 / \mathrm{N}$ & $H$ & $14.03 \pm 0.20$ & $4.041 \pm 0.003$ & $87.20 \pm 0.20$ & $4.046 \pm 0.007$ & $86.87 \pm 0.20$ & 40.4 & BG \\
\hline 1RXS J193528.9+374605 & $\mathrm{CC} 4$ & 2010 Aug 17 & 2010.6249 & $\mathrm{NIRC} 2 / \mathrm{N}$ & $H$ & $15.90 \pm 0.98$ & $4.070 \pm 0.003$ & $89.21 \pm 0.20$ & $\ldots$ & $\ldots$ & 40.7 & $\ldots$ \\
\hline 1RXS J193528.9+374605 & $\mathrm{CC} 4$ & 2011 Aug 20 & 2011.6333 & $\mathrm{NIRC} 2 / \mathrm{N}$ & $H$ & $15.69 \pm 0.78$ & $4.213 \pm 0.003$ & $88.23 \pm 0.20$ & $4.212 \pm 0.007$ & $87.86 \pm 0.20$ & 42.1 & BG \\
\hline 1RXS J193528.9+374605 & $\mathrm{CC} 5$ & 2010 Aug 17 & 2010.6249 & $\mathrm{NIRC} 2 / \mathrm{N}$ & $H$ & $14.97 \pm 0.49$ & $4.006 \pm 0.003$ & $210.25 \pm 0.20$ & $\ldots$ & $\ldots$ & 40.1 & $\ldots$ \\
\hline 1RXS J193528.9+374605 & CC5 & 2011 Aug 20 & 2011.6333 & $\mathrm{NIRC} 2 / \mathrm{N}$ & $H$ & $15.05 \pm 0.40$ & $3.863 \pm 0.003$ & $209.36 \pm 0.20$ & $3.848 \pm 0.006$ & $209.22 \pm 0.23$ & 38.6 & BG \\
\hline 1RXS J193528.9+374605 & CC6 & 2010 Aug 17 & 2010.6249 & $\mathrm{NIRC} 2 / \mathrm{N}$ & $H$ & $15.85 \pm 1.00$ & $5.134 \pm 0.003$ & $238.69 \pm 0.20$ & & & 51.3 & \\
\hline 1RXS J193528.9+374605 & CC6 & 2011 Aug 20 & 2011.6333 & $\mathrm{NIRC} 2 / \mathrm{N}$ & $H$ & $16.16 \pm 1.07$ & $4.971 \pm 0.003$ & $239.02 \pm 0.20$ & $4.962 \pm 0.007$ & $238.86 \pm 0.22$ & 49.7 & BG \\
\hline 1RXS J193528.9+374605 & $\mathrm{CC} 7$ & 2010 Aug 17 & 2010.6249 & $\mathrm{NIRC} 2 / \mathrm{N}$ & $H$ & $15.07 \pm 0.59$ & $4.329 \pm 0.003$ & $262.36 \pm 0.20$ & & $\ldots$ & 43.3 & $\ldots$ \\
\hline 1RXS J193528.9+374605 & $\mathrm{CC} 7$ & 2011 Aug 20 & 2011.6333 & $\mathrm{NIRC} 2 / \mathrm{N}$ & $H$ & $15.31 \pm 0.57$ & $4.191 \pm 0.003$ & $263.51 \pm 0.20$ & $4.178 \pm 0.007$ & $263.49 \pm 0.21$ & 41.9 & BG \\
\hline 1RXS J193528.9+374605 & $\mathrm{CC} 8$ & 2010 Aug 17 & 2010.6249 & $\mathrm{NIRC} 2 / \mathrm{N}$ & $H$ & $10.77 \pm 0.30$ & $4.437 \pm 0.003$ & $281.59 \pm 0.20$ & $\ldots$ & $\ldots$ & 44.4 & $\ldots$ \\
\hline 1RXS J193528.9+374605 & $\mathrm{CC} 8$ & 2011 Aug 20 & 2011.6333 & $\mathrm{NIRC} 2 / \mathrm{N}$ & $H$ & $10.83 \pm 0.15$ & $4.322 \pm 0.003$ & $283.31 \pm 0.20$ & $4.323 \pm 0.007$ & $283.28 \pm 0.21$ & 43.2 & BG \\
\hline 1RXS J193528.9+374605 & CC9 & 2011 Aug 20 & 2011.6333 & $\mathrm{NIRC} 2 / \mathrm{N}$ & $H$ & $16.10 \pm 1.06$ & $3.088 \pm 0.003$ & $129.53 \pm 0.20$ & $\ldots$ & $\ldots$ & 30.9 & SE \\
\hline 2MASS J19435432-0546363 & $\mathrm{CC} 1$ & 2012 May 21 & 2012.3875 & $\mathrm{NIRC} 2 / \mathrm{N}$ & $K_{S}$ & $11.11 \pm 0.33$ & $4.321 \pm 0.003$ & $108.66 \pm 0.20$ & $\ldots$ & & 138.3 & \\
\hline 2MASS J19435432-0546363 & $\mathrm{CC} 1$ & 2013 May 2 & 2013.3330 & $\mathrm{NIRC} 2 / \mathrm{N}$ & $K_{S}$ & $10.64 \pm 0.12$ & $4.236 \pm 0.003$ & $109.52 \pm 0.20$ & $4.267 \pm 0.008$ & $108.97 \pm 0.23$ & 135.6 & BG \\
\hline 2MASS J19435432-0546363 & $\mathrm{CC} 2$ & 2012 May 21 & 2012.3875 & $\mathrm{NIRC} 2 / \mathrm{N}$ & $K_{S}$ & $11.78 \pm 0.63$ & $5.284 \pm 0.003$ & $192.63 \pm 0.20$ & $\ldots$ & $\ldots$ & 169.1 & SE \\
\hline 2MASS J19435432-0546363 & $\mathrm{CC} 3$ & 2012 May 21 & 2012.3875 & $\mathrm{NIRC} 2 / \mathrm{N}$ & $K_{S}$ & $11.70 \pm 0.57$ & $2.405 \pm 0.003$ & $244.72 \pm 0.20$ & $\ldots$ & $\ldots$ & 77.0 & $\ldots$ \\
\hline 2MASS J19435432-0546363 & $\mathrm{CC} 3$ & 2013 May 2 & 2013.3330 & $\mathrm{NIRC} 2 / \mathrm{N}$ & $K_{S}$ & $11.06 \pm 0.13$ & $2.471 \pm 0.003$ & $244.92 \pm 0.20$ & $2.461 \pm 0.008$ & $245.20 \pm 0.27$ & 79.1 & BG \\
\hline 2MASS J19435432-0546363 & $\mathrm{CC} 4$ & 2012 May 21 & 2012.3875 & $\mathrm{NIRC} 2 / \mathrm{N}$ & $K_{S}$ & $11.61 \pm 0.49$ & $4.849 \pm 0.003$ & $281.46 \pm 0.20$ & & & 155.2 & \\
\hline 2MASS J19435432-0546363 & $\mathrm{CC} 4$ & 2013 May 2 & 2013.3330 & $\mathrm{NIRC} 2 / \mathrm{N}$ & $K_{S}$ & $\ldots \mathrm{f}$ & $4.912 \pm 0.003$ & $281.20 \pm 0.20$ & $4.898 \pm 0.008$ & $281.26 \pm 0.22$ & 157.2 & BG \\
\hline 2MASS J19435432-0546363 & CC5 & 2013 May 2 & 2013.3330 & $\mathrm{NIRC} 2 / \mathrm{N}$ & $K_{S}$ & $13.50 \pm 1.01$ & $5.971 \pm 0.003$ & $82.84 \pm 0.20$ & 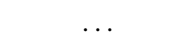 & 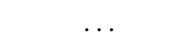 & 191.1 & SE \\
\hline 2MASS J19435432-0546363 & CC6 & 2013 May 2 & 2013.3330 & $\mathrm{NIRC} 2 / \mathrm{N}$ & $K_{S}$ & $13.61 \pm 1.04$ & $4.851 \pm 0.003$ & $94.24 \pm 0.20$ & $\cdots$ & $\ldots$ & 155.2 & SE \\
\hline 2MASS J19435432-0546363 & $\mathrm{CC} 7$ & 2013 May 2 & 2013.3330 & $\mathrm{NIRC} 2 / \mathrm{N}$ & $K_{S}$ & $13.44 \pm 0.99$ & $5.790 \pm 0.003$ & $332.96 \pm 0.20$ & $\ldots$ & $\ldots$ & 185.3 & SE \\
\hline NLTT 48651 & $\mathrm{CC} 1$ & 2011 Jun 21 & 2011.4694 & NIRC $2 / \mathrm{N}$ & $H$ & $10.22 \pm 0.16$ & $2.273 \pm 0.003$ & $252.47 \pm 0.20$ & $\ldots$ & $\ldots$ & 22.7 & $\ldots$ \\
\hline NLTT 48651 & $\mathrm{CC} 1$ & 2012 May 21 & 2012.3876 & $\mathrm{NIRC} 2 / \mathrm{N}$ & $K_{S}$ & $9.99 \pm 0.73$ & $2.340 \pm 0.003$ & $260.78 \pm 0.20$ & $2.346 \pm 0.010$ & $260.62 \pm 0.25$ & 23.4 & BG \\
\hline NLTT 48651 & $\mathrm{CC} 2$ & 2011 Jun 21 & 2011.4694 & $\mathrm{NIRC} 2 / \mathrm{N}$ & $H$ & $10.67 \pm 0.16$ & $6.131 \pm 0.003$ & $0.86 \pm 0.20$ & $\ldots$ & $\ldots$ & 61.3 & \\
\hline NLTT 48651 & $\mathrm{CC} 2$ & 2012 May 21 & 2012.3876 & $\mathrm{NIRC} 2 / \mathrm{N}$ & $K_{S}$ & $10.37 \pm 0.52$ & $6.431 \pm 0.003$ & $359.76 \pm 0.20$ & $6.433 \pm 0.008$ & $359.50 \pm 35.78$ & 64.3 & BG \\
\hline 2MASS J20284361-1128307 & $\mathrm{CC} 1$ & 2012 May 10 & 2012.3577 & HiCIAO & $H$ & $7.48 \pm 0.05$ & $5.644 \pm 0.021$ & $38.68 \pm 0.24$ & $\ldots$ & $\ldots$ & 79.0 & $\ldots$ \\
\hline 2MASS J20284361-1128307 & $\mathrm{CC} 1$ & 2013 Aug 17 & 2013.6254 & $\mathrm{NIRC} 2 / \mathrm{W}$ & $K_{S}$ & $\ldots \mathrm{c}$ & $5.704 \pm 0.074$ & $36.80 \pm 0.20$ & $5.675 \pm 0.026$ & $36.88 \pm 0.29$ & 79.9 & BG \\
\hline
\end{tabular}


Table 8

(Continued)

\begin{tabular}{|c|c|c|c|c|c|c|c|c|c|c|c|c|}
\hline \multirow[b]{2}{*}{ Name } & \multirow[b]{2}{*}{ Cand. } & \multirow[b]{2}{*}{$\begin{array}{l}\text { UT Date } \\
\text { (Y-M-D) }\end{array}$} & \multirow[b]{2}{*}{ Epoch } & \multirow[b]{2}{*}{ Instrument $^{\mathrm{a}}$} & \multirow[b]{2}{*}{ Filt. } & \multirow[b]{2}{*}{$\begin{array}{c}\text { Contrast } \\
\text { (mag) }\end{array}$} & \multicolumn{2}{|c|}{ Measured Position } & \multicolumn{2}{|c|}{ Background Position } & \multirow[b]{2}{*}{$\begin{array}{l}\text { Proj. Sep. } \\
\text { (AU) }\end{array}$} & \multirow[b]{2}{*}{ Comp.? ${ }^{\mathrm{b}}$} \\
\hline & & & & & & & $\begin{array}{c}\rho \\
\left({ }^{\prime \prime}\right)\end{array}$ & $\begin{array}{l}\text { P.A. } \\
\left({ }^{\circ}\right)\end{array}$ & $\begin{array}{c}\rho \\
\left(^{\prime \prime}\right)\end{array}$ & $\begin{array}{l}\text { P.A. } \\
\left({ }^{\circ}\right)\end{array}$ & & \\
\hline 2MASS J20284361-1128307 & $\mathrm{CC} 2$ & 2012 May 10 & 2012.3577 & HiCIAO & $H$ & $10.22 \pm 0.12$ & $3.073 \pm 0.020$ & $128.96 \pm 0.39$ & & & 43.0 & \\
\hline 2MASS J20284361-1128307 & $\mathrm{CC} 2$ & 2013 Aug 17 & 2013.6254 & $\mathrm{NIRC} 2 / \mathrm{W}$ & $K_{S}$ & $\ldots{ }^{c}$ & $2.834 \pm 0.074$ & $128.39 \pm 0.20$ & $2.895 \pm 0.025$ & $128.42 \pm 0.54$ & 39.7 & BG \\
\hline 2MASS J20284361-1128307 & $\mathrm{CC} 3$ & 2012 May 10 & 2012.3577 & HiCIAO & $H$ & $11.02 \pm 0.25$ & $5.579 \pm 0.020$ & $231.00 \pm 0.24$ & $\ldots$ & $\ldots$ & 78.1 & \\
\hline 2MASS J20284361-1128307 & $\mathrm{CC} 3$ & 2013 Aug 17 & 2013.6254 & $\mathrm{NIRC} 2 / \mathrm{W}$ & $K_{S}$ & $\ldots{ }^{c}$ & $5.632 \pm 0.074$ & $233.89 \pm 0.20$ & $5.594 \pm 0.028$ & $232.84 \pm 0.27$ & 78.8 & BG \\
\hline 2MASS J20284361-1128307 & $\mathrm{CC} 4$ & 2013 Aug 17 & 2013.6254 & $\mathrm{NIRC} 2 / \mathrm{W}$ & $K_{S}$ & $\ldots{ }^{c}$ & $21.666 \pm 0.077$ & $81.81 \pm 0.20$ & $\ldots$ & $\ldots$ & 303.3 & SE \\
\hline 2MASS J20284361-1128307 & $\mathrm{CC} 5$ & 2013 Aug 17 & 2013.6254 & NIRC2/W & $K_{S}$ & $\ldots{ }^{c}$ & $20.218 \pm 0.077$ & $95.04 \pm 0.20$ & $\ldots$ & $\ldots$ & 283.1 & SE \\
\hline 2MASS J20284361-1128307 & CC6 & 2013 Aug 17 & 2013.6254 & NIRC2/W & $K_{S}$ & $\ldots{ }^{c}$ & $20.209 \pm 0.077$ & $100.03 \pm 0.20$ & $\ldots$ & $\ldots$ & 282.9 & SE \\
\hline 2MASS J20284361-1128307 & $\mathrm{CC} 7$ & 2013 Aug 17 & 2013.6254 & NIRC2/W & $K_{S}$ & $\ldots{ }^{c}$ & $12.948 \pm 0.075$ & $110.23 \pm 0.20$ & $\ldots$ & $\ldots$ & 181.3 & SE \\
\hline 2MASS J20284361-1128307 & $\mathrm{CC} 8$ & 2013 Aug 17 & 2013.6254 & NIRC2/W & $K_{S}$ & $\ldots{ }^{\mathrm{c}}$ & $21.073 \pm 0.077$ & $122.73 \pm 0.20$ & $\ldots$ & $\ldots$ & 295.0 & SE \\
\hline 2MASS J20284361-1128307 & $\mathrm{CC} 9$ & 2013 Aug 17 & 2013.6254 & NIRC2/W & $K_{S}$ & $\ldots{ }^{c}$ & $19.537 \pm 0.077$ & $327.34 \pm 0.20$ & $\ldots$ & & 273.5 & SE \\
\hline GJ 4186 B & $\mathrm{CC} 1$ & 2010 Aug 17 & 2010.6255 & $\mathrm{NIRC} 2 / \mathrm{N}$ & $H$ & $14.32 \pm 0.85$ & $3.251 \pm 0.003$ & $231.45 \pm 0.20$ & & & 62.8 & \\
\hline GJ 4186 B & $\mathrm{CC} 1$ & 2011 Jun 21 & 2011.4695 & $\mathrm{NIRC} 2 / \mathrm{N}$ & $H$ & $14.53 \pm 1.00$ & $3.451 \pm 0.003$ & $233.43 \pm 0.20$ & $3.455 \pm 0.004$ & $233.35 \pm 0.19$ & 66.6 & BG \\
\hline GJ 4185 Aab & $\mathrm{CC} 1$ & 2011 Jun 21 & 2011.4696 & $\mathrm{NIRC} 2 / \mathrm{N}$ & $H$ & $12.27 \pm 0.76$ & $3.586 \pm 0.003$ & $76.42 \pm 0.20$ & $\ldots$ & $\ldots$ & 69.9 & $\ldots$ \\
\hline GJ 4185 Aab & $\mathrm{CC} 1$ & 2012 May 21 & 2012.3876 & $\mathrm{NIRC} 2 / \mathrm{N}$ & $K_{S}$ & $11.31 \pm 0.76$ & $3.355 \pm 0.003$ & $76.59 \pm 0.20$ & $3.387 \pm 0.004$ & $75.92 \pm 0.21$ & 65.4 & BG \\
\hline GJ 4185 Aab & $\mathrm{CC} 2$ & 2011 Jun 21 & 2011.4696 & $\mathrm{NIRC} 2 / \mathrm{N}$ & $H$ & $10.58 \pm 0.31$ & $2.449 \pm 0.003$ & $195.69 \pm 0.20$ & $\ldots$ & $\ldots$ & 47.8 & $\ldots$ \\
\hline GJ 4185 Aab & $\mathrm{CC} 2$ & 2012 May 21 & 2012.3876 & $\mathrm{NIRC} 2 / \mathrm{N}$ & $K_{S}$ & $9.56 \pm 0.22$ & $2.590 \pm 0.003$ & $199.51 \pm 0.20$ & $2.528 \pm 0.004$ & $199.95 \pm 0.20$ & 50.5 & BG \\
\hline GJ 4185 Aab & $\mathrm{CC} 3$ & 2011 Jun 21 & 2011.4696 & $\mathrm{NIRC} 2 / \mathrm{N}$ & $H$ & $11.51 \pm 0.28$ & $4.192 \pm 0.003$ & $225.30 \pm 0.20$ & $\ldots$ & & 81.7 & \\
\hline GJ 4185 Aab & $\mathrm{CC} 3$ & 2012 May 21 & 2012.3876 & $\mathrm{NIRC} 2 / \mathrm{N}$ & $K_{S}$ & $11.08 \pm 0.48$ & $4.402 \pm 0.003$ & $226.19 \pm 0.20$ & $4.350 \pm 0.004$ & $226.99 \pm 0.21$ & 85.8 & BG \\
\hline NLTT 54873 & $\mathrm{CC} 1$ & 2010 Aug 18 & 2010.6279 & $\mathrm{NIRC} 2 / \mathrm{N}$ & $H$ & $7.07 \pm 0.16$ & $3.616 \pm 0.003$ & $292.70 \pm 0.20$ & & & 79.6 & \\
\hline NLTT 54873 & $\mathrm{CC} 1$ & 2011 Aug 20 & 2011.6337 & $\mathrm{NIRC} 2 / \mathrm{N}$ & $H$ & $7.45 \pm 0.19$ & $3.747 \pm 0.003$ & $290.29 \pm 0.20$ & $3.738 \pm 0.009$ & $290.33 \pm 0.24$ & 82.4 & BG \\
\hline GJ 4337 A & $\mathrm{CC} 1$ & 2010 Aug 18 & 2010.6281 & $\mathrm{NIRC} 2 / \mathrm{N}$ & $H$ & $13.62 \pm 0.34$ & $4.174 \pm 0.003$ & $242.55 \pm 0.20$ & $\ldots$ & $\ldots$ & 62.2 & $\ldots$ \\
\hline GJ $4337 \mathrm{~A}$ & $\mathrm{CC} 1$ & 2011 Aug 20 & 2011.6338 & $\mathrm{NIRC} 2 / \mathrm{N}$ & $H$ & $13.78 \pm 0.29$ & $4.515 \pm 0.003$ & $245.27 \pm 0.20$ & $4.530 \pm 0.009$ & $245.44 \pm 0.21$ & 67.3 & BG \\
\hline GJ 1290 & $\mathrm{CC} 1$ & 2011 Nov 15 & 2011.8713 & $\mathrm{NIRC} 2 / \mathrm{N}$ & $H$ & $14.12 \pm 0.50$ & $3.772 \pm 0.003$ & $132.26 \pm 0.20$ & & & 83.0 & \\
\hline GJ 1290 & $\mathrm{CC} 1$ & 2012 Oct 8 & 2012.7701 & $\mathrm{NIRC} 2 / \mathrm{N}$ & $H$ & $13.24 \pm 1.13$ & $3.527 \pm 0.003$ & $138.01 \pm 0.20$ & $3.530 \pm 0.008$ & $137.87 \pm 0.25$ & 77.6 & BG \\
\hline G 68-46 & $\mathrm{CC} 1$ & 2012 Aug 23 & 2012.6447 & $\mathrm{NIRC} 2 / \mathrm{N}$ & $K_{S}$ & $12.46 \pm 0.60$ & $2.246 \pm 0.003$ & $27.62 \pm 0.20$ & $\ldots$ & $\ldots$ & 47.2 & $\ldots$ \\
\hline G $68-46$ & $\mathrm{CC} 1$ & 2013 Aug 17 & 2013.6255 & $\mathrm{NIRC} 2 / \mathrm{N}$ & $K_{S}$ & $12.58 \pm 0.34$ & $2.205 \pm 0.003$ & $20.29 \pm 0.20$ & $2.204 \pm 0.009$ & $20.63 \pm 0.28$ & 46.3 & BG \\
\hline G $68-46$ & $\mathrm{CC} 2$ & 2012 Aug 23 & 2012.6447 & $\mathrm{NIRC} 2 / \mathrm{N}$ & $K_{S}$ & $13.50 \pm 1.07$ & $3.090 \pm 0.003$ & $100.93 \pm 0.20$ & $\ldots$ & $\ldots$ & 64.9 & $\ldots$ \\
\hline G 68-46 & $\mathrm{CC} 2$ & 2013 Aug 17 & 2013.6255 & $\mathrm{NIRC} 2 / \mathrm{N}$ & $K_{S}$ & $13.92 \pm 1.05$ & $2.827 \pm 0.003$ & $99.89 \pm 0.20$ & $2.816 \pm 0.008$ & $100.54 \pm 0.27$ & 59.4 & BG \\
\hline G 68-46 & $\mathrm{CC} 3$ & 2012 Aug 23 & 2012.6447 & $\mathrm{NIRC} 2 / \mathrm{N}$ & $K_{S}$ & $13.56 \pm 1.15$ & $4.261 \pm 0.003$ & $41.82 \pm 0.20$ & $\ldots$ & & 89.5 & \\
\hline G 68-46 & $\mathrm{CC} 3$ & 2013 Aug 17 & 2013.6255 & $\mathrm{NIRC} 2 / \mathrm{N}$ & $K_{S}$ & $13.48 \pm 0.86$ & $4.148 \pm 0.003$ & $38.54 \pm 0.20$ & $4.145 \pm 0.009$ & $38.43 \pm 0.24$ & 87.1 & BG \\
\hline G 68-46 & $\mathrm{CC} 4$ & 2012 Aug 23 & 2012.6447 & $\mathrm{NIRC} 2 / \mathrm{N}$ & $K_{S}$ & $12.95 \pm 0.91$ & $4.332 \pm 0.003$ & $51.67 \pm 0.20$ & $\ldots$ & & 91.0 & $B G^{e}$ \\
\hline G 68-46 & $\mathrm{CC} 4$ & 2013 Aug 17 & 2013.6255 & $\mathrm{NIRC} 2 / \mathrm{N}$ & $K_{S}$ & $13.58 \pm 0.90$ & $4.195 \pm 0.003$ & $48.12 \pm 0.20$ & $4.175 \pm 0.009$ & $48.66 \pm 0.23$ & 88.1 & BG \\
\hline 1RXS J235452.2+383129 & $\mathrm{CC} 1$ & 2011 Aug 20 & 2011.6337 & $\mathrm{NIRC} 2 / \mathrm{N}$ & $H$ & $13.80 \pm 0.41$ & $5.304 \pm 0.003$ & $236.79 \pm 0.20$ & $\ldots$ & $\ldots$ & 84.3 & $\ldots$ \\
\hline 1RXS J235452.2+383129 & $\mathrm{CC} 1$ & 2012 Aug 23 & 2012.6448 & $\mathrm{NIRC} 2 / \mathrm{N}$ & $H$ & $13.93 \pm 1.04$ & $5.145 \pm 0.003$ & $236.58 \pm 0.20$ & $5.143 \pm 0.009$ & $236.76 \pm 0.22$ & 81.8 & BG \\
\hline
\end{tabular}

Notes.

${ }^{\text {a }}$ NIRC2/N refers to the narrow $\left(\approx 10^{\prime \prime} \times 10^{\prime \prime}\right)$ camera mode; NIRC2/W refers to the wide $\left(\approx 40^{\prime \prime} \times 40^{\prime \prime}\right)$ camera.

${ }^{\mathrm{b}} \mathrm{BG}=$ background object; $\mathrm{SE}=$ single epoch detection; $\mathrm{AMB}=$ ambiguous whether comoving or background.

${ }^{c}$ The primary and/or companion is saturated or in the nonlinear regime of the infrared array, so no relative photometry is listed.

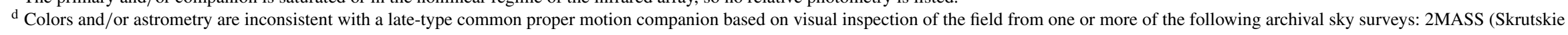
et al. 2006), SDSS (Abazajian et al. 2009), DSS1, or DSS2.

e Source appears spatially extended and is probably a background galaxy.

${ }^{\mathrm{f}}$ Source is near the edge of the array so no relative photometry is given.

(This table is also available in a machine-readable form) 

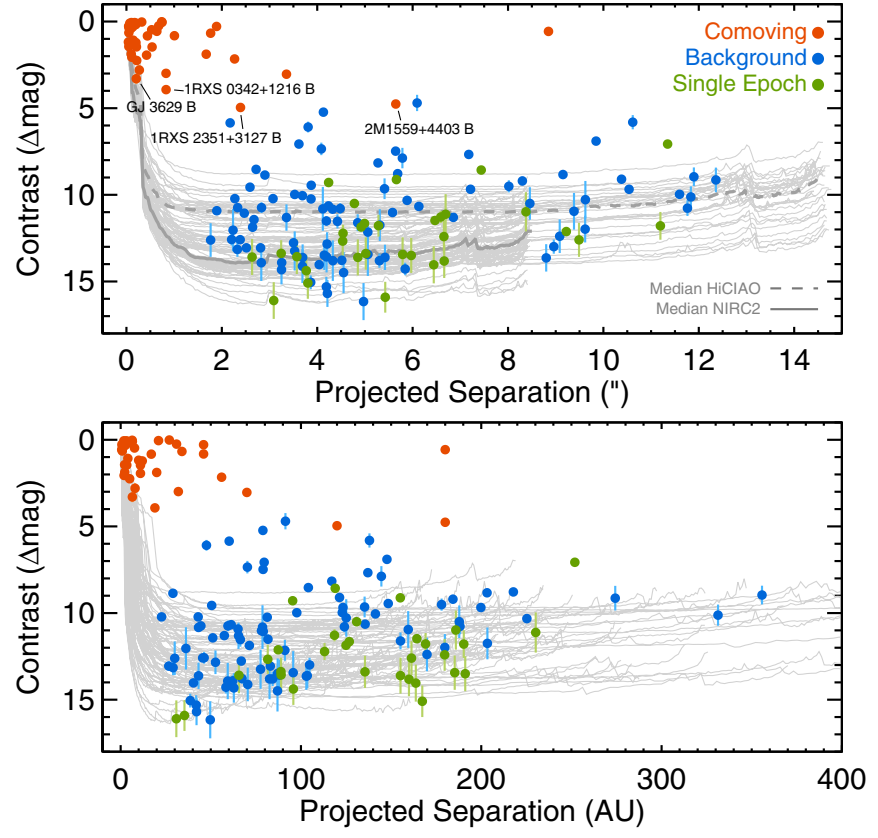

Figure 19. Point sources detected in our survey. Comoving stellar and substellar companions are shown in red, background objects are plotted in blue, and objects with only a single epoch of astrometry are in green. The individual and median NIRC2 and HiCIAO contrasts are overplotted in gray. In the top panel the measured angular separations and contrasts are displayed, while the projected separation is used in the bottom panel. For clarity we have excluded several dozen single epoch candidates in the single crowded field surrounding GJ 9652 A.

where $\theta, \rho$, and $\sigma$ are the measured and predicted P.A., separation, and their associated uncertainties at epoch $i$ for $N$ epochs of astrometry. $v$ is the number of degrees of freedom, equal to $2 \times N-1$. For the background case, the predicted measurements incorporate the proper motion and distance to the target as listed in Table 3. For the co-moving scenario, the predicted P.A. and separation assume no orbital motion as expected for companions on wide orbits.

Table 9 summarizes our tests for common proper motion for candidates with at least two epochs of astrometry. 93 candidates are consistent with background stars. The status of

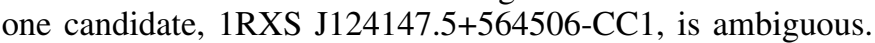
The remaining 73 only have a single epoch of astrometry. We do not identify any planets in our sample.

\section{STATISTICAL ANALYSIS}

Our null detection of planets provides powerful constraints on the outer architecture of planetary systems around low-mass stars. Likewise, our four brown dwarf discoveries allow us to measure the frequency of brown dwarf companions to $M$ dwarfs over a variety of separations. Since we are not uniformly sensitive to companions in mass and separation, a sensitivity map for each target must be considered. Similarly, our brown dwarf discoveries were made in projected separation and their distributions in semi-major axis space must be inferred to derive accurate statistical constraints. In the following analysis, we assume two forms of eccentricity distributions to test their influence on our results: circular orbits $(e=0)$ and eccentricities following a $1-e$ distribution, in which most planets have small or modest eccentricities (see Section 6.3). The latter case is motivated by the distribution of radial velocity detected planets (Kipping 2013) and M dwarf binaries (Duchêne \& Kraus
2013). Below we describe two approaches to derive statistical constraints over a range of companion masses and separations.

\subsection{Contrast Curve Selection Guidelines}

A common complication of large direct imaging surveys is that not all faint planet candidates can be followed up with second-epoch astrometry. Finite telescope time, different seeing conditions and $\mathrm{AO}$ correction, and varying FOV rotation can both prevent candidates found in first-epoch imaging from being recovered and reveal new fainter or wider point sources. In this survey we found that 93 out of 167 faint point sources are stationary background stars. Care must therefore be taken in our statistical treatment of the remaining 74 candidates with unclear status. Note, however, that only 8 of these are within projected separations of $100 \mathrm{AU}$ around single stars in our sample and most of them (42) come from a single low-galactic latitude target.

Following the recipe of Nielsen et al. (2013), we define selection guidelines for choosing contrast curves to use in our statistical analysis. These are considered on a case-by-case basis for each target in our survey.

1. If no candidates are identified in a first-epoch observation and no subsequent deeper imaging is obtained, then the contrast curve is used for our statistical analysis.

(a) If a later, deeper epoch of imaging uncovers candidates that are shown to be background, this deeper contrast curve is used.

(b) If a later, deeper epoch uncovers candidates that are not shown to be background, then the initial candidate-free first epoch contrast curve is used.

2. If one or more candidates are identified at the first epoch and are shown to be background objects from subsequent imaging, and no other candidates are identified in the follow-up observation, then the deeper of the two contrasts is used.

(a) If a second epoch reveals additional candidates that only have a single epoch of astrometry, then the first epoch contrast curve is used.

(b) If a second epoch fails to recover one or more candidates then this second epoch contrast curve is used. This is analogous to (1b) but in reverse order.

3. If only a single first-epoch observation is obtained and one or more candidates are identified then the floor of the contrast curve is defined to be $2 \sigma$ above the brightest candidate with unknown status, where $\sigma$ is the uncertainty in the relative contrast of that candidate. Since we have no information about whether single-epoch candidates are background or comoving, the raw contrast curve cannot be included in the statistical analysis. Instead, we homogeneously remove all information about companions below the threshold of the brightest single-epoch candidate in the image.

\subsection{Wide Stellar Binaries}

Wide stellar binaries beyond $\sim 100$ AU can dramatically influence the outer regions of planetary systems by creating dynamically unstable zones where planets cannot exist on long timescales. These wide binaries must therefore be taken into account in the statistical analysis of the survey. Table 10 lists the multiplicity properties of the sample. Altogether, 25 of our targets have companions beyond $100 \mathrm{AU}$. 
Table 9

Common Proper Motion Tests

\begin{tabular}{|c|c|c|c|c|c|c|c|}
\hline Name & Cand. & $\begin{array}{l}\text { No. of } \\
\text { Epochs }\end{array}$ & $\begin{array}{l}\Delta t \\
(\mathrm{yr})\end{array}$ & $\begin{array}{c}\chi_{v}^{2} \\
(\mathrm{BG})\end{array}$ & $\begin{array}{c}\chi_{v}^{2} \\
(\mathrm{CPM})\end{array}$ & $v$ & Comp.? \\
\hline GJ $3030 \mathrm{AB}$ & $\mathrm{CC} 1$ & 2 & 1.64 & 0.57 & 17.08 & 3 & BG \\
\hline GJ $3030 \mathrm{AB}$ & $\mathrm{CC} 2$ & 2 & 1.64 & 2.31 & 434960 & 3 & BG \\
\hline NLTT 1875 & $\mathrm{CC} 1$ & 2 & 1.01 & 2.74 & 203.5 & 3 & BG \\
\hline NLTT 1875 & $\mathrm{CC} 2$ & 2 & 1.01 & 2.28 & 344.2 & 3 & BG \\
\hline NLTT 1875 & $\mathrm{CC} 3$ & 2 & 1.01 & 0.49 & 866.9 & 3 & BG \\
\hline NLTT 1875 & $\mathrm{CC} 4$ & 2 & 1.01 & 1.62 & 279.5 & 3 & BG \\
\hline NLTT 1875 & $\mathrm{CC} 5$ & 2 & 1.01 & 2.30 & 63.77 & 3 & BG \\
\hline NLTT 1875 & CC6 & 2 & 1.01 & 3.14 & 72.03 & 3 & BG \\
\hline LP 247-13 & $\mathrm{CC} 1$ & 5 & 0.97 & 1.47 & 30.24 & 9 & BG \\
\hline NLTT 13844 & $\mathrm{CC} 1$ & 2 & 0.27 & 0.66 & 668.6 & 3 & BG \\
\hline NLTT 13844 & $\mathrm{CC} 2$ & 2 & 0.27 & 0.07 & 634.3 & 3 & BG \\
\hline 1RXS J045101.0+312734 & $\mathrm{CC} 1$ & 3 & 0.93 & 0.11 & 6.07 & 5 & BG \\
\hline 1RXS J045101.0+312734 & $\mathrm{CC} 2$ & 2 & 0.93 & 0.07 & 6.93 & 3 & BG \\
\hline AP Col & $\mathrm{CC} 1$ & 3 & 0.78 & 2.09 & 26.90 & 5 & BG \\
\hline G 108-36 & $\mathrm{CC} 1$ & 2 & 0.93 & 0.01 & 7.33 & 3 & BG \\
\hline G 108-36 & $\mathrm{CC} 2$ & 2 & 0.93 & 0.02 & 563613 & 3 & BG \\
\hline G $108-36$ & $\mathrm{CC} 3$ & 2 & 0.93 & 0.04 & 11.17 & 3 & BG \\
\hline G $108-36$ & $\mathrm{CC} 4$ & 2 & 0.93 & 0.25 & 5.88 & 3 & BG \\
\hline G $108-36$ & $\mathrm{CC} 5$ & 2 & 0.93 & 0.21 & 4.41 & 3 & BG \\
\hline 1RXS J073829.3+240014 & $\mathrm{CC} 1$ & 2 & 2.14 & 1.88 & 3274.7 & 3 & BG \\
\hline NLTT 26359 & $\mathrm{CC} 1$ & 2 & 1.35 & 0.23 & 19.98 & 3 & BG \\
\hline NLTT 26359 & $\mathrm{CC} 2$ & 2 & 1.35 & 0.20 & 8.98 & 3 & BG \\
\hline G $13-33 \mathrm{AB}$ & $\mathrm{CC} 1$ & 2 & 1.10 & 3.14 & 49.05 & 3 & BG \\
\hline G $13-33 \mathrm{AB}$ & $\mathrm{CC} 2$ & 2 & 0.71 & 3.49 & 128.1 & 3 & BG \\
\hline GJ $3729 \mathrm{AB}$ & $\mathrm{CC} 1$ & 2 & 0.94 & 0.94 & 9.01 & 3 & BG \\
\hline GJ $3729 \mathrm{AB}$ & $\mathrm{CC} 2$ & 2 & 0.54 & 1.08 & 19.17 & 3 & BG \\
\hline 1RXS J124147.5+564506 & $\mathrm{CC} 1$ & 2 & 0.98 & 2.35 & 0.11 & 3 & $\mathrm{AMB}$ \\
\hline 2MASS J14124864-1629561 & $\mathrm{CC} 1$ & 2 & 0.94 & 0.20 & 308.4 & 3 & BG \\
\hline G $166-49$ & $\mathrm{CC} 1$ & 2 & 0.71 & 0.53 & 523.4 & 3 & BG \\
\hline G $166-49$ & $\mathrm{CC} 2$ & 2 & 0.71 & 0.23 & 1140 & 3 & BG \\
\hline G $166-49$ & $\mathrm{CC} 3$ & 2 & 0.71 & 1.13 & 951.7 & 3 & BG \\
\hline 2MASS J15323737+4653048 & $\mathrm{CC} 1$ & 2 & 1.23 & 1.47 & 420.82 & 3 & BG \\
\hline GJ $3966 \mathrm{AB}$ & $\mathrm{CC} 1$ & 2 & 0.81 & 8.27 & 104.3 & 3 & BG \\
\hline G 669 A & $\mathrm{CC} 1$ & 2 & 0.98 & 2.52 & 56.39 & 3 & BG \\
\hline G 669 A & $\mathrm{CC} 2$ & 2 & 0.98 & 0.68 & 29.51 & 3 & BG \\
\hline G 669 A & $\mathrm{CC} 3$ & 2 & 0.98 & 2.38 & 8.60 & 3 & BG \\
\hline G 669 A & $\mathrm{CC} 4$ & 2 & 0.98 & 2.87 & 8.44 & 3 & BG \\
\hline G 669 A & $\mathrm{CC} 5$ & 2 & 0.98 & 2.13 & 11.55 & 3 & BG \\
\hline LHS 3321 & $\mathrm{CC} 1$ & 2 & 0.89 & 2.24 & 178.4 & 3 & BG \\
\hline GJ 9652 A & $\mathrm{CC} 1$ & 2 & 1.01 & 0.03 & 6587 & 3 & BG \\
\hline GJ $9652 \mathrm{~A}$ & $\mathrm{CC} 2$ & 2 & 1.01 & 0.00 & 1921 & 3 & BG \\
\hline GJ 9652 A & $\mathrm{CC} 3$ & 2 & 1.01 & 0.01 & 1387 & 3 & BG \\
\hline GJ $9652 \mathrm{~A}$ & $\mathrm{CC} 4$ & 2 & 1.01 & 0.35 & 1820 & 3 & BG \\
\hline GJ $9652 \mathrm{~A}$ & $\mathrm{CC} 5$ & 2 & 1.01 & 0.08 & 3431 & 3 & BG \\
\hline GJ 9652 A & CC6 & 2 & 1.01 & 0.03 & 3733 & 3 & BG \\
\hline GJ 9652 A & $\mathrm{CC} 7$ & 2 & 1.01 & 0.60 & 2834 & 3 & BG \\
\hline GJ $9652 \mathrm{~A}$ & CC10 & 2 & 1.01 & 0.49 & 1321 & 3 & BG \\
\hline GJ 9652 A & CC13 & 2 & 1.01 & 0.07 & 8967 & 3 & BG \\
\hline GJ 9652 A & CC18 & 2 & 1.01 & 0.00 & 7737 & 3 & BG \\
\hline GJ $9652 \mathrm{~A}$ & CC19 & 2 & 1.01 & 0.13 & 6736 & 3 & BG \\
\hline GJ $9652 \mathrm{~A}$ & $\mathrm{CC} 20$ & 2 & 1.01 & 0.04 & 4129 & 3 & BG \\
\hline GJ 9652 A & $\mathrm{CC} 21$ & 2 & 1.01 & 0.00 & 10728 & 3 & BG \\
\hline GJ $9652 \mathrm{~A}$ & $\mathrm{CC} 22$ & 2 & 1.01 & 0.03 & 10139 & 3 & BG \\
\hline 2MASS J19303829-1335083 & $\mathrm{CC} 1$ & 2 & 0.87 & 0.37 & 1387 & 3 & BG \\
\hline 2MASS J19303829-1335083 & $\mathrm{CC} 2$ & 2 & 0.87 & 0.56 & 846.6 & 3 & BG \\
\hline 2MASS J19303829-1335083 & $\mathrm{CC} 3$ & 2 & 0.87 & 3.97 & 2091 & 3 & BG \\
\hline 2MASS J19303829-1335083 & $\mathrm{CC} 4$ & 2 & 0.87 & 1.08 & 2242 & 3 & BG \\
\hline 1RXS J193528.9+374605 & $\mathrm{CC} 1$ & 2 & 1.01 & 1.20 & 53.81 & 3 & BG \\
\hline 1RXS J193528.9+374605 & $\mathrm{CC} 2$ & 2 & 1.01 & 0.58 & 393.9 & 3 & BG \\
\hline 1RXS J193528.9+374605 & $\mathrm{CC} 3$ & 2 & 1.01 & 0.60 & 362.3 & 3 & BG \\
\hline 1RXS J193528.9+374605 & $\mathrm{CC} 4$ & 2 & 1.01 & 0.58 & 382.7 & 3 & BG \\
\hline 1RXS J193528.9+374605 & $\mathrm{CC} 5$ & 2 & 1.01 & 1.74 & 382.0 & 3 & BG \\
\hline 1RXS J193528.9+374605 & CC6 & 2 & 1.01 & 0.56 & 492.5 & 3 & BG \\
\hline 1RXS J193528.9+374605 & $\mathrm{CC} 7$ & 2 & 1.01 & 0.97 & 358.2 & 3 & BG \\
\hline
\end{tabular}


Table 9

(Continued)

\begin{tabular}{lccccccc}
\hline \hline Name & Cand. & $\begin{array}{c}\text { No. of } \\
\text { Epochs }\end{array}$ & $\begin{array}{c}\Delta t \\
(\mathrm{yr})\end{array}$ & $\begin{array}{c}\chi_{v}^{2} \\
(\mathrm{BG})\end{array}$ & $\begin{array}{c}\chi_{v}^{2} \\
(\mathrm{CPM})\end{array}$ & $v$ & Comp.? \\
\hline 1RXS J193528.9+374605 & CC8 & 2 & 1.01 & 0.01 & 257.2 & 3 & BG \\
2MASS J19435432-0546363 & CC1 & 2 & 0.95 & 5.47 & 136.9 & 3 & BG \\
2MASS J19435432-0546363 & CC3 & 2 & 0.95 & 0.69 & 80.83 & 3 & BG \\
2MASS J19435432-0546363 & CC4 & 2 & 0.95 & 0.91 & 73.78 & 3 & BG \\
NLTT 48651 & CC1 & 2 & 0.92 & 0.19 & 370.9 & 3 & BG \\
NLTT 48651 & CC2 & 2 & 0.92 & 0.02 & 538371 & 3 & BG \\
2MASS J20284361-1128307 & CC1 & 2 & 1.27 & 0.06 & 12.27 & 3 & BG \\
2MASS J20284361-1128307 & CC2 & 2 & 1.27 & 0.20 & 3.80 & 3 & BG \\
2MASS J20284361-1128307 & CC3 & 2 & 1.27 & 3.33 & 28.68 & 3 & BG \\
GJ 4186 B & CC1 & 2 & 0.84 & 0.24 & 757.1 & 3 & BG \\
GJ 4185 Aab & CC1 & 2 & 0.92 & 15.43 & 988.3 & 3 & BG \\
GJ 4185 Aab & CC2 & 2 & 0.92 & 52.06 & 429.0 & 3 & BG \\
GJ 4185 Aab & CC3 & 2 & 0.92 & 38.59 & 820.0 & 3 & BG \\
NLTT 54873 & CC1 & 2 & 1.01 & 0.31 & 342.0 & 3 & BG \\
GJ 4337 A & CC1 & 2 & 1.01 & 0.95 & 2184 & 3 & BG \\
GJ 1290 & CC1 & 2 & 0.90 & 0.10 & 1249 & 3 & BG \\
G 68-46 & CC1 & 2 & 0.98 & 0.33 & 255.0 & 3 & BG \\
G 68-46 & CC2 & 2 & 0.98 & 1.80 & 1285 & 3 & BG \\
G 68-46 & CC3 & 2 & 0.98 & 0.07 & 281.2 & 3 & BG \\
G 68-46 & CC4 & 2 & 0.98 & 2.53 & 400.1 & 3 & BG \\
1RXS J235452.2+383129 & CC1 & 2 & 1.01 & 0.14 & 468.35 & 3 & BG \\
\hline
\end{tabular}

Our treatment of wide binaries follows that of Nielsen et al. (2013) and is based on simulations by Holman \& Wiegert (1999) of stability zones surrounding close-in planets with a wide stellar companion (S-type orbits) and wide-separation circumbinary planets (P-type orbits). Holmam \& Wiegert show that the region of stable orbits is a strong function of both binary eccentricity and mass ratio. The eccentricity distribution of these (very) wide-separation binaries is unknown because of their long orbital periods. However, Abt (2006) showed that eccentricities become increasingly uniform (i.e., random) at long periods $\left(10^{5}-10^{6}\right.$ days $)$, with a mean eccentricity tending to 0.5 . We therefore adopt eccentricities of 0.5 for wide binary companions in our sample. Assuming equal mass stars, the critical semi-major axis for stable S-type orbits from Holman $\&$ Wiegert (1999) is $\approx 10 \%$ of the stellar semi-major axis. For P-type orbits, the inner stability limit is $\sim 4$ times the binary semi-major axis.

Wide binaries in our sample are complicated by projection effects and their unknown current orbital phase. We therefore adopt a median conversion factor of 1.14 from Dupuy \& Liu (2011) for the case of no discovery bias to transform projected separations into semi-major axes. For our statistical analysis, we then assume that the region between $10 \%$ and $400 \%$ of the binary semi-major axis is devoid of planets and does not contribute any information to our statistical analysis. These allowable regions are listed in Table 10. As described above, these are conservative stability limits assuming a star-wide binary companion eccentricity of 0.5 and coplanar binary and planetary orbits. Relaxing these constraints would provide more room for dynamically stable planets to reside. Indeed, Tokovinin et al. (2006) found the empirical limit for dynamical stability of triple star systems is near period ratios of 5 (that is, $P_{3} / P_{1}$ $>5$ ), which may be a more realistic boundary. Note that we do not exclude regions surrounding our brown dwarf discoveries for dynamical reasons since we had no a priori knowledge of their existence. In this way our sensitivity maps for these targets contribute to the number of trials and the discoveries contribute to the number of detections in these regions (see Section 6.4).

\subsection{Mass Sensitivity}

Converting contrast curves into sensitivity limits in planet mass and separation requires the use of substellar evolutionary models. These cooling curves in turn depend on assumptions about the way in which planets form. "Hot-start" models (e.g., Burrows et al. 1997; Saumon \& Marley 2008) slowly radiate their initial gravitational potential energy over time and therefore best represent formation via disk instability (e.g., Boss 1997; Mayer 2002). On the other hand, "cold-start" and "warmstart" models (e.g., Marley et al. 2007; Spiegel \& Burrows 2012; Mollière \& Mordasini 2012; Bodenheimer et al. 2013) follow a core accretion prescription, which assumes significant loss of initial entropy at formation through punctuated energy dissipation associated with accretion events. In addition to differences in initial conditions, though to a lesser degree, assumptions about the atmospheric properties of giant planets can also influence both the rate at which planets cool and the evolution of their spectra (e.g., Chabrier et al. 2000).

We adopt four sets of evolutionary models for this survey to reflect uncertainties in the formation and atmospheric properties of giant planets. Our choices are based on the accuracy of the models in reproducing the observed colors of brown dwarfs and giant planets and on the sampling of the various publicly available grids in mass and age. The properties of all four grids are summarized in Figure 20. We selected solar-metallicity hot-start models incorporating three general prescriptions of photospheric dust: (1) the Cond models of Baraffe et al. (2003), in which dust is modeled as having already formed and settled below the photosphere; (2) the Dusty models of Chabrier et al. (2000), which present an extreme view of photospheric dust formation and retention at all temperatures; and (3) the BT-Settl isochrones from Allard et al. (2011), which simulate the growth and sedimentation of dust across the M/L/T transitions. The Cond models are well sampled from ages of $1 \mathrm{Myr}$ to $10 \mathrm{Gyr}$ and 
Table 10

Sample Multiplicity Properties

\begin{tabular}{|c|c|c|c|c|c|c|c|}
\hline Name & Type $^{\mathrm{a}}$ & $\begin{array}{l}\text { Wide Comp. } \\
\text { Name }\end{array}$ & $\begin{array}{c}\rho \\
\left({ }^{\prime \prime}\right)\end{array}$ & $\begin{array}{l}\text { Proj. Sep. } \\
\quad(A U)\end{array}$ & $\begin{array}{l}\text { Orbital Period } \\
(\mathrm{yrs})\end{array}$ & $\begin{array}{c}\text { Allowable Planet } a \\
(\mathrm{AU})\end{array}$ & $\begin{array}{l}\text { Discovery } \\
\text { Reference }\end{array}$ \\
\hline G 217-32 AB & CVB & $\ldots$ & 0.5 & 7.7 & 48 & $>35$ & B14 \\
\hline 1RXS J001557.5-163659 AB & CVB & $\ldots$ & 0.1 & 1.8 & 4.5 & $>8$ & B14 \\
\hline GJ $3030 \mathrm{AB}$ & MVB & $\ldots$ & 2.2 & 56 & 1000 & $<6,>260$ & M01 \\
\hline G 132-50 Aab & $\mathrm{PMB}+\mathrm{CVB} ; \mathrm{WVB}$ & G132-51 Bab & $0.3 ; 26$ & $8 ; 770$ & $32 ; 25000$ & $36-88,>3500$ & M05, B14; G63 \\
\hline G 271-110 & WVB & EX Cet & 610 & 14600 & 2500000 & $<1700,>67000$ & AF11 \\
\hline G 272-115 & SB2 & $\ldots$ & $\ldots$ & $\ldots$ & $\ldots$ & $\ldots$ & Sh15 \\
\hline NLTT 6549 AB & CVB & $\ldots$ & 0.05 & 2.2 & 4.2 & $>10$ & B14 \\
\hline 2MASS J03033668-2535329 AB & $\mathrm{PMB}+\mathrm{CVB}$ & $\ldots$ & 0.8 & 32 & 210 & $>150$ & M05, J12 \\
\hline 1RXS J034231.8+121622 AB & CVB & $\ldots$ & 0.8 & 19 & 76 & $\ldots$ & $\mathrm{J} 12, \mathrm{~B} 14$ \\
\hline G 160-54 ABC & $\mathrm{CVB}+\mathrm{SB} 3 ; \mathrm{MVB}$ & $\ldots$ & $0.2 ; 3.3$ & $4 ; 70$ & $17 ; 1300$ & $>320$ & B14, Sh15 \\
\hline 2MASS J04220833-2849053 AB & CVB & $\ldots$ & 0.8 & 21 & 120 & $>100$ & B14 \\
\hline 2MASS J04472312-2750358 & MVB & 2MASS J04472266-2750295 & 8.8 & 180 & 3200 & $<21,>820$ & M01 \\
\hline G 81-34 AB & CVB & $\ldots$ & 0.6 & 14 & 95 & $>64$ & B14 \\
\hline L 449-1 AB & CVB & $\ldots$ & 0.05 & 0.6 & $2.5^{\mathrm{d}}$ & $>0.3$ & Ri14 \\
\hline GJ 3371 A & WVB & GJ 3372 B & 160 & 2170 & 140000 & $<250,>9900$ & G66a \\
\hline GJ 3372 B & WVB & GJ $3371 \mathrm{~A}$ & 160 & 2170 & 140000 & $<250,>9900$ & G66a \\
\hline 2MASS J06131330-2742054 AB & $\mathrm{CVB}+\mathrm{SB} 2$ & $\ldots$ & 0.09 & 2.7 & 8.3 & $>12$ & Ri14, Sh15 \\
\hline LHS $1864 \mathrm{AB}$ & $\mathrm{MVB}+\mathrm{SB} 2$ & $\ldots$ & 1.8 & 34 & 320 & $<27,>1100$ & M01, Sh15 \\
\hline 1RXS J091744.5+461229 AB & CVB & $\ldots$ & 0.2 & 6.5 & 20 & $>30$ & $\mathrm{~J} 12$ \\
\hline GJ $354.1 \mathrm{~B}$ & WVB & DX Leo & 65 & 1160 & 52000 & $<130,>5300$ & G98 \\
\hline PYC J09362+3731 AB & SB2 & $\ldots$ & $\ldots$ & $\ldots$ & $\ldots$ & $\ldots$ & Sc12c \\
\hline NLTT 22741 A & WVB & 2MASS J09510549+3558021 & 13 & 400 & 20000 & $<46,>1800$ & $\operatorname{Re} 06$ \\
\hline GJ 3577 A & WVB & GJ 3578 B & 23 & 570 & 23000 & $<65,>2600$ & G63 \\
\hline GJ $3578 \mathrm{~B}$ & WVB & GJ 3577 A & 23 & 570 & 23000 & $<65,>2600$ & G63 \\
\hline G 196-3 A & WVB & G 196-3 B & 16 & 180 & 4000 & $<20,>820$ & $\operatorname{Re} 98$ \\
\hline GJ 2079 AB & $\mathrm{PMB}+\mathrm{CVB}+\mathrm{SB} 1$ & $\ldots$ & 0.1 & 2.3 & 4.8 & $>10$ & M05, B14, Sh12 \\
\hline GJ 3629 AB & CVB & $\ldots$ & 0.2 & 6.5 & 30 & $\ldots$ & B12a \\
\hline PYC J10571+0544 AB & MVB & $\ldots$ & 1.0 & 46 & 780 & $>210$ & B14 \\
\hline 2MASS J11240434+3808108 & MVB & 2MASS J11240487+3808054 & 8.3 & 170 & 5100 & $<19,>780$ & $\mathrm{Cr} 03$ \\
\hline TWA $30 \mathrm{~A}$ & WVB & TWA $30 \mathrm{~B}$ & 80 & 3370 & 560000 & $<380,>15000$ & Lo10 \\
\hline 2MASS J12062214-1314559 AB & CVB & $\ldots$ & 0.4 & 11 & 55 & $>50$ & $\mathrm{~J} 12$ \\
\hline G $13-33$ AB & CVB & $\ldots$ & 0.1 & 1.5 & 4.7 & $>7$ & B14 \\
\hline LP 735-48 AB & MVB & $\ldots$ & 1.9 & 46 & 470 & $>210$ & B14 \\
\hline GJ $3729 \mathrm{AB}$ & CVB & $\ldots$ & 0.05 & 0.9 & 2.4 & $>4$ & B14 \\
\hline 2MASS J12383713-2703348 AB & CVB & $\ldots$ & 0.2 & 5 & 19 & $>23$ & B14 \\
\hline GJ $490 \mathrm{Bab}$ & CVB; WVB & GJ $490 \mathrm{Aab}$ & $0.2 ; 16$ & $3.3 ; 310$ & $16 ; 13000$ & $13-35,>1400$ & B14; G64 \\
\hline GJ $490 \mathrm{Aab}$ & CVB; WVB & GJ $490 \mathrm{Bab}$ & $0.1 ; 16$ & $1.9 ; 310$ & $7.3 ; 13000$ & $8-35,>1400$ & B14; G64 \\
\hline NLTT 34410 AB & $\mathrm{CVB}^{\mathrm{c}}$ & $\ldots$ & 0.1 & 3 & 11 & $>14$ & B14 \\
\hline 2MASS J14215503-3125537 AB & CVB & $\ldots$ & 0.07 & 2.8 & 8.4 & $>13$ & B14 \\
\hline 1RXS J150907.2+590422 A & MVB & 2MASS J15090696+5904282 & 9.0 & 300 & 8500 & $<34,>1400$ & M01 \\
\hline LHS 3122 AB & CVB & $\ldots$ & 0.2 & 3.4 & 12 & $>16$ & B14 \\
\hline 2MASS J15594729+4403595 AB & MVB & $\ldots$ & 5.6 & 180 & 3800 & $<21,>820$ & $\mathrm{~J} 12$ \\
\hline 2MASS J16074132-1103073 AB & CVB & $\ldots$ & 0.7 & 27 & 240 & $>120$ & M14 \\
\hline GJ 3966 AB & CVB & $\ldots$ & 0.09 & 1.5 & 3.4 & $>7$ & B14 \\
\hline GJ 3997 AB & MVB & $\ldots$ & 1.7 & 20 & 150 & $>91$ & B14 \\
\hline LP 447-38 AB & CVB & $\ldots$ & 0.4 & 10 & 52 & $>46$ & B14 \\
\hline GJ 669 B & WVB & GJ 669 A & 17 & 200 & 4100 & $<23,>910$ & G64 \\
\hline GJ 669 A & WVB & GJ 669 B & 17 & 200 & 4100 & $<23,>910$ & G64 \\
\hline GJ 9652 A & SB1; WVB & GJ 9652 B & 40 & 780 & 30000 & $<89,>3600$ & Sh12; G66a \\
\hline 1RXS J193528.9+374605 & SB1 & $\ldots$ & $\ldots$ & $\ldots$ & $\ldots$ & $\ldots$ & Sh12 \\
\hline 2MASS J19560294-3207186 AB & CVB; WVB & TYC 7443-1102-1 & $0.2 ; 26$ & $12 ; 1530$ & $66 ; 72000$ & $55-170,>7000$ & B14; L09 \\
\hline TYC 7443-1102-1 & WVB & 2MASS J19560294-3207186 AB & 26 & 1530 & 72000 & $<170,>7000$ & L09 \\
\hline 2MASS J20003177+5921289 AB & CVB & $\ldots$ & 0.3 & 6.4 & 34 & $>29$ & $\mathrm{~J} 12$ \\
\hline 2MASS J20100002-2801410 AB & CVB & $\ldots$ & 0.7 & 31 & 290 & $>140$ & B10 \\
\hline NLTT 50066 AB & CVB & $\ldots$ & 0.14 & 5.3 & 17 & $>24$ & $\mathrm{~J} 12$ \\
\hline NLTT 50710 & SB2 & $\ldots$ & $\ldots$ & $\ldots$ & $\ldots$ & $\ldots$ & Sh15 \\
\hline GJ 4186 B & WVB & GJ 4185 Aab & 26 & 510 & 18000 & $<58,>2300$ & P94 \\
\hline GJ 4185 Aab & CVB; WVB & GJ 4186 B & $0.05 ; 26$ & $1.0 ; 510$ & $1.6 ; 18000$ & $<58,>2300$ & B14; P94 \\
\hline PYC J21376+0137 AB & CVB & $\ldots$ & 0.4 & 17 & 140 & $>78$ & B14 \\
\hline 1RXS J221419.3+253411 AB & CVB & $\ldots$ & 0.1 & 4 & 23 & $>18$ & B14 \\
\hline
\end{tabular}


Table 10

(Continued)

\begin{tabular}{|c|c|c|c|c|c|c|c|}
\hline Name & Type $^{\mathrm{a}}$ & $\begin{array}{l}\text { Wide Comp. } \\
\text { Name }\end{array}$ & $\begin{array}{c}\rho \\
(")\end{array}$ & $\begin{array}{l}\text { Proj. Sep. } \\
\text { (AU) }\end{array}$ & $\begin{array}{l}\text { Orbital Period } \\
\text { (yrs) }\end{array}$ & $\begin{array}{l}\text { Allowable Planet } a \\
\text { (AU) }\end{array}$ & $\begin{array}{l}\text { Discovery } \\
\text { Reference }\end{array}$ \\
\hline GJ 4338 Bab & CVB; WVB & GJ 4337 A & $0.05 ; 17.6$ & $0.7 ; 260$ & $1.0 ; 6200$ & $<30,>1200$ & B14; G66b \\
\hline GJ 4337 A & WVB & GJ 4338 Bab & 17.6 & 260 & 6200 & $<30,>1200$ & G66b \\
\hline GJ $4381 \mathrm{AB}$ & CVB & $\ldots$ & 0.5 & 60 & 11 & $>50$ & MZB01 \\
\hline
\end{tabular}

Notes.

${ }^{\text {a }} \mathrm{CVB}=$ close visual binary $\left(<1^{\prime \prime}\right)$; MVB = moderate visual binary $\left(1-10^{\prime \prime}\right)$; WVB = wide visual binary $\left(>10^{\prime \prime}\right)$; SB1 = single-line spectroscopic binary; SB2 = double-line spectroscopic binary; SB3 = triple-line spectroscopic binary; PMB = proper motion binary.

${ }^{\mathrm{b}}$ Dynamically stable regions from Holman \& Wiegert (1999) assuming an equal-mass binary with an eccentricity of 0.5. Stable zones within 5 AU are excluded because we lack the sensitivity to detect planets in this region.

c The Washington Double Star Catalog lists LP 232-169 as a wide companion to NLTT 34410 AB at 172". However, Scholz et al. (2005b) find LP 232-169 is a K2 subdwarf at a distance of $\sim 400-800 \mathrm{pc}$; they also find that the proper motions significantly disagree. These stars appear to be unrelated.

${ }^{\mathrm{d}}$ See Riedel et al. (2014).

References. (AF11) Alonso-Floriano et al. 2011; (B12a) Bowler et al. 2012a; (B12b) Bowler et al. 2012b; (B14) this work; (Cr03) Cruz et al. 2003; (G63) Giclas et al. 1963; (G64) Giclas et al. 1964; (G66a) Giclas et al. 1966a; (G66b) Giclas et al. 1966b; (G98) Gaidos 1998; (J12) Janson et al. 2012; (M01) Mason et al. 2001; (M05) Makarov \& Kaplan 2005; (M14) Malo et al. 2014a; (MZB01) McCarthy et al. 2001; (Lo10) Looper et al. 2010a; (L09) Lépine \& Simon 2009; (P94) Poveda et al. 1994; (Re98) Rebolo et al. 1998; (Ri14) Riedel et al. 2014; (Sc12b) Schlieder et al. 2012c; (Sh12) Shkolnik et al. 2012; (Sh15) E. L. Shkolnik et al. (in preparation); (Sk14) B. Skiff (2013, private communication).
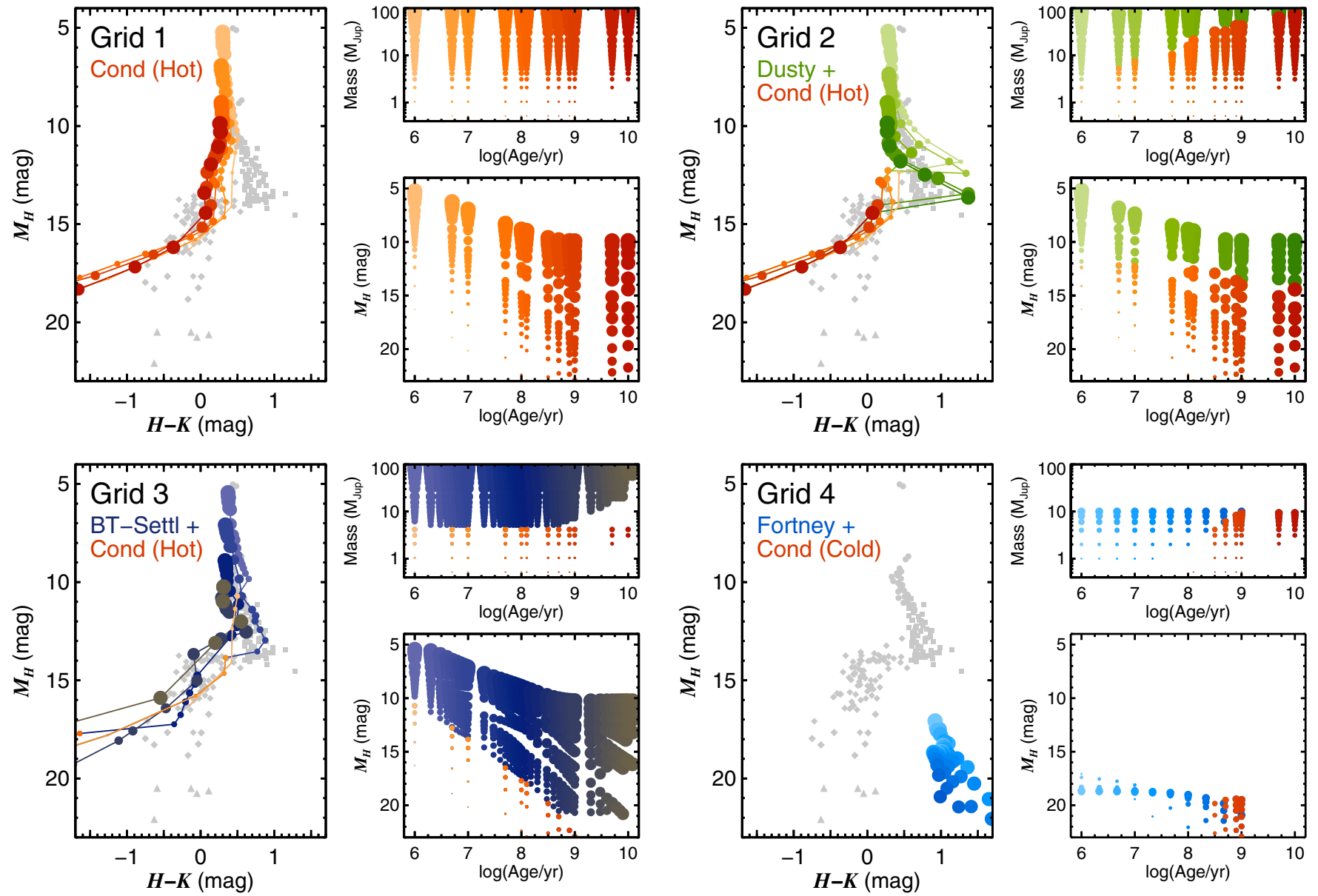

Figure 20. Evolutionary model grids used in this work. Three of the cases (Grids 1-3) are based on a hot-start formation scenario, while the fourth (Grid 4) follows a cold-start prescription. Each three-panel set shows the predicted color-magnitude sequence in $M_{H}$ vs. $H-K$ compared to the observed sequence of MLTY dwarfs (gray; from Dupuy \& Liu 2012 and Dupuy \& Kraus 2013), the grid sampling in age and mass, and the evolution of $M_{H}$ with time. Mass ranges of 0.5-100 $M_{\text {Jup }}$ (0.5-10 $M_{\text {Jup }}$ ) are shown for hot-start (cold-start) cases with symbol sizes scaling with mass. Grid 1 shows the Cond models of Baraffe et al. (2003), which poorly reproduce dusty L dwarfs and mid-to late-T dwarfs in color. Grid 2 is a hybrid of Dusty models from Chabrier et al. (2000) above $1500 \mathrm{~K}$ and Cond models at lower temperatures. Grid 3 shows the BT-Settl models from Allard et al. (2011) above $5 M_{\text {Jup }}$ and the Cond grid at lower masses, producing the best fit to the M, L, and early T sequence. The cold-start scenario with slight (5x solar) metal-enrichment from Fortney et al. (2008) is shown in Grid 4 and is supplemented with Cond models at older ages. The three hot-start cases predict similar evolution of absolute magnitude with planet mass and, overall, produce very similar statistical results in this study. 
masses of $0.5 M_{\mathrm{Jup}}$-to $0.1 M_{\odot}$. Dusty models produce better fits to the L dwarf color-magnitude sequence, but are too red below about $1500 \mathrm{~K}$ (Figure 20); we therefore supplement the Dusty grid with Cond models below that temperature, resulting in a "Dusty+Cond" combination. The BT-Settl models do a better job reproducing the $\mathrm{M}, \mathrm{L}$, and $\mathrm{T}$ sequence, but are not uniformly sampled at very low masses; we therefore supplement that grid with Cond models below $5 M_{\text {Jup }}$. For the cold-start models we adopt the grid from Fortney et al. (2008), which assumes slight metal enrichment (five times solar abundances), includes masses below $13 M_{\text {Jup }}$, and focuses on relatively young ages ( $\lesssim 1$ Gyr). At older ages, all planetary-mass objects should have temperatures below $\sim 600 \mathrm{~K}$, so we supplement the Fortney grid with Cond models in that region.

Our strategy to infer planet detectability for each target in the \{planet mass, semi-major axis \} plane is based on Monte Carlo realizations of simulated planets on random orbits. For a given target and semi-major axis $a$ we generate $10^{4}$ orbits projected onto the sky with random ascending node position angles, arguments of periastron, orbital inclinations (drawn from a $\sin i$ distribution), and periastron passage times. We consider two possible eccentricity distributions, $e=0$ and $P(e)$ $\propto 1-e$, to test whether adding modest eccentricities affects the results. This choice of the eccentricity distribution is motivated by observations of intermediate-period (100-10,000 day) $\mathrm{M}$ dwarf binaries and extrasolar giant planets measured from radial velocity surveys, which have similar distribution shapes that peak at small eccentricities and diminish roughly linearly to high values (Duchêne \& Kraus 2013; Kipping 2013).

For a given companion mass $m$ we use the star's distance and age together with evolutionary models to assign an apparent magnitude to each simulated companion. Gaussian age distributions are adopted for stars that belong to YMGs, while linearly uniform distributions are used for the rest (see Table 2). The uncertainty in the distance is also incorporated as a Gaussian distribution. This allows us to then compare the apparent magnitudes and sky-projected separations of all orbits for a given $\{m, a\}$ to our contrast curves. The fraction of simulated companions that fall above the curve (the "detections") is the overall sensitivity at that grid point. Fractional FOV coverage is also incorporated by randomly assigning "non-detections" to planets with a probability equal to 1 minus the azimuthal coverage at that separation.

These simulations are repeated for all grid steps in mass (from 0.5-100 $M_{\text {Jup }}$ ) and physical separation (1-1000 AU), all four sets of evolutionary models, both circular orbits and eccentricity distributions following $P(e) \propto 1-e$, and our three methods of PSF subtraction. For our statistical analysis we adopt contrast curves from the aggressive version of the LOCI reduction because overall they produce the best contrasts, but the resulting mass sensitivities are similar for all cases. Figure 21 shows the distribution of contrasts for our three PSF subtraction methods. At $1^{\prime \prime}$, the aggressive implementation of LOCI outperforms the scaled median subtraction and our conservative version of LOCI by $0.5_{-0.3}^{+0.7} \mathrm{mag}$ and $0.12_{-0.17}^{+0.20} \mathrm{mag}$, respectively. However, this gain in contrast is only marginal in planet mass: for the typical age of our sample $(\approx 125 \mathrm{Myr})$, the Cond models of Baraffe et al. (2003) predict an $H$-band brightness difference between a 9 and $10 M_{\text {Jup }}$ (4 and $5 M_{\text {Jup }}$ ) planet of 0.50 mag $(0.71 \mathrm{mag})$. Our three hot-start model prescriptions (Cond, Dusty+Cond, BT-Settl+Cond) produce similar sensitivity maps, so for the rest of this work we show representative results with Cond models.
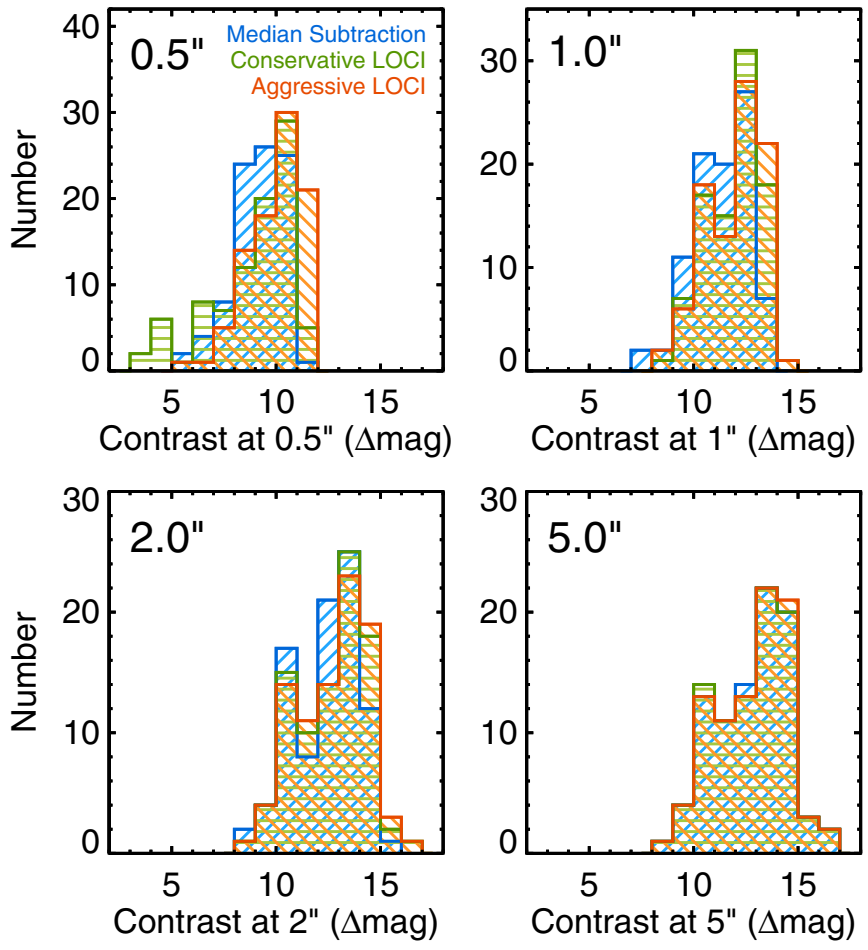

Figure 21. Comparison of three methods of PSF subtraction between 0.'5-5" Scaled median subtraction (blue) systematically produces slightly worse $(7 \sigma)$ contrasts compared to conservative (green) and aggressive (red) applications of LOCI. At separations of $0 . .5$ near the IWA, the median subtraction outperforms conservative LOCI when sky rotation is small. Beyond $3^{\prime \prime}$ where the data are no longer contrast-limited, the median scaled subtraction method is used for all contrasts and the distributions are virtually identical.

As an example, Figure 22 shows Cond and Fortney sensitivity maps for G 271-110 based on the contrasts for this target. As expected, our data are not sensitive to planetary companions within $\sim 10$ AU nor any companions beyond a few hundred AU because of the limited FOV coverage. In this case most planets in the 10-100 AU range would have been detected. In general, introducing non-zero eccentricities tends to slightly "smear out" the sensitivity plots, but the overall impact is small.

Finally, we note that our sensitivity maps are necessarily dependent on substellar cooling models, which remain poorly constrained by observations. In the few instances where they have been tested through precise dynamical mass measurements of the benchmark brown dwarf systems HD 130948 BC and Gl 417 BC, Dupuy et al. $(2009,2014)$ found that low-mass evolutionary models systematically overpredict brown dwarf masses by $\approx 15 \%-25 \%$. A similar result was found by Crepp et al. (2012) with the older HR 7672 AB system. This potential (and worrisome) uncalibrated systematic error in cooling models is much larger than any effects caused by our choice of eccentricity distribution or PSF subtraction method.

\subsection{Giant Planet Frequency at a Given Planet Mass}

Our first approach focuses on the following question: for a given planet mass and semi-major axis, what planet frequency is consistent with the non-detection from our survey? Since we did not detect any planets, this analysis is concerned with the (95\% confidence) upper limits on planet frequencies. We use the sensitivity maps for each target (Section 6.3) to compute the overall $95 \%$ confidence upper limit at each $\{m, a\}$ grid point. For a given $m$ and $a$, the number of detections $N_{\text {det }}$ is 

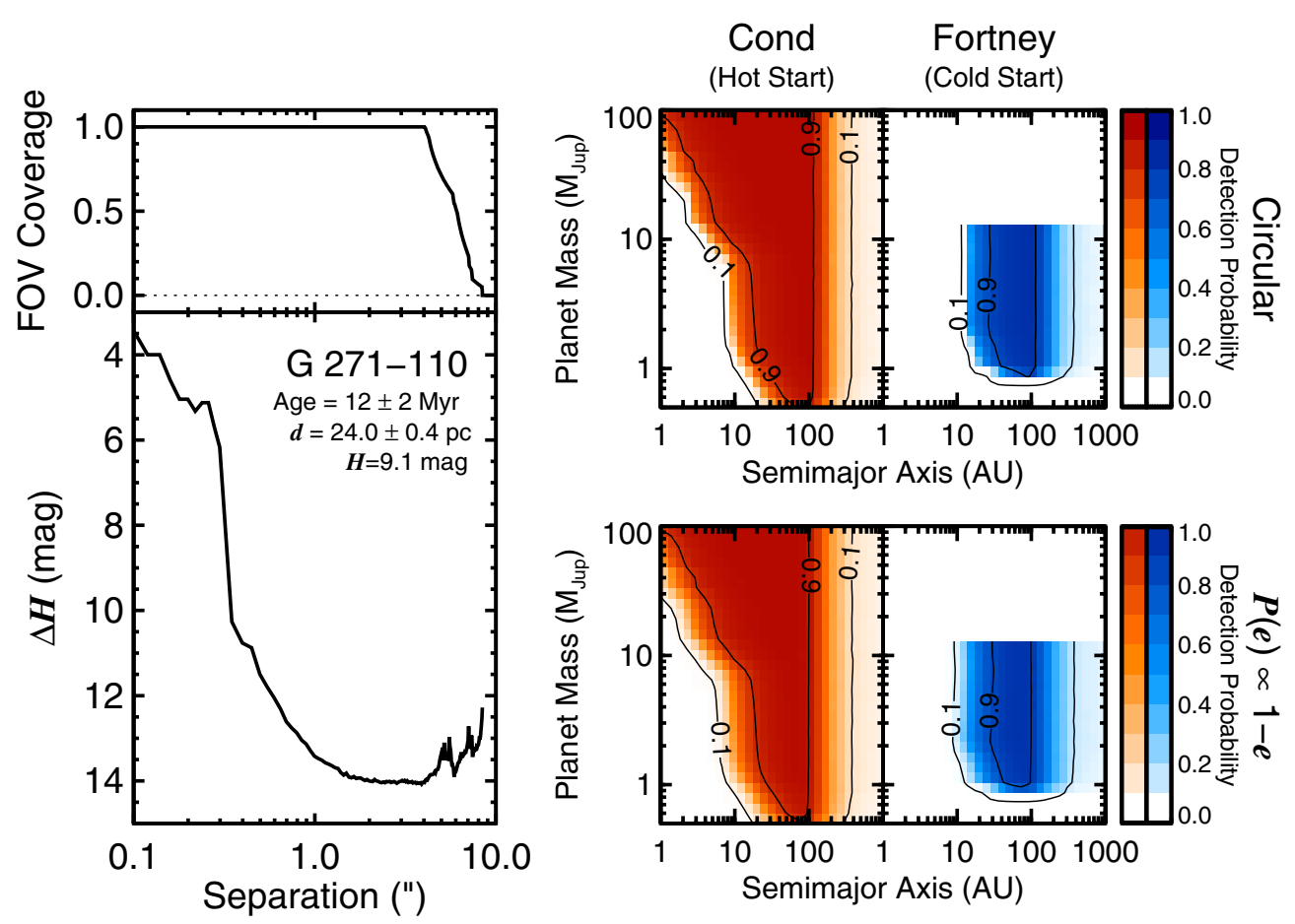

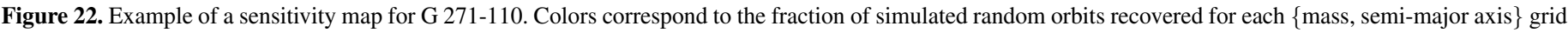

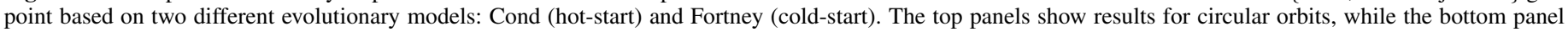
follows a $1-e$ eccentricity distribution.

uniformly zero and the effective number of trials $N_{\text {trials }}$ is simply the sum of the sensitivities at that grid point $(s(m, a))$ over all targets $N_{\text {tar }}$ :

$$
N_{\text {trials }}=\sum_{i=1}^{N_{\text {tar }}} s_{i}(m, a),
$$

where $s$ is a number from 0 to 1 derived by the methods in Section 6.3. Since these constitute Bernoulli trials we can compute the probability distribution of the occurrence rate $f$ using the binomial distribution. In a region $100 \%$ sensitive to companions for all of our targets, the number of trials would simply be equal to the number of targets, and the commonly used binomial distribution applies. On the other hand, for noninteger trials and successes the binomial coefficient can be generalized using Gamma functions. The binomial distribution then becomes

$$
P(f \mid n, k)=\frac{\Gamma(n+1)}{\Gamma(k+1) \Gamma(n-k+1)} f^{k}(1-f)^{n-k}(n+1),
$$

where $n$ is the number of trials and $k$ is the number of successes. The final $(n+1)$ factor is a normalization constant. ${ }^{11}$ This is similar to the widely used method from Nielsen et al. (2008), but here we use the more general binomial distribution instead of the Poisson distribution, which is only applicable for cases when $N_{\text {trials }}$ is large and $f$ is small. For regions in $\{m, a\}$ where the sensitivity to planets is low (small separations, large separations, and low masses), $N_{\text {trials }}$ is small so the binomial distribution must be used to accurately measure upper limits.

Figure 23 shows the results for the Cond and Fortney models with two assumptions about the planet eccentricity distributions.

\footnotetext{
11 The meaning of a "trial" and "success" becomes less intuitive with continuous rather than integer values. However, noting that $\Gamma(x+1)=x$ ! for integer values of $x$, Equation (7) reduces to its usual form when $k$ and $n$ are natural numbers.
}
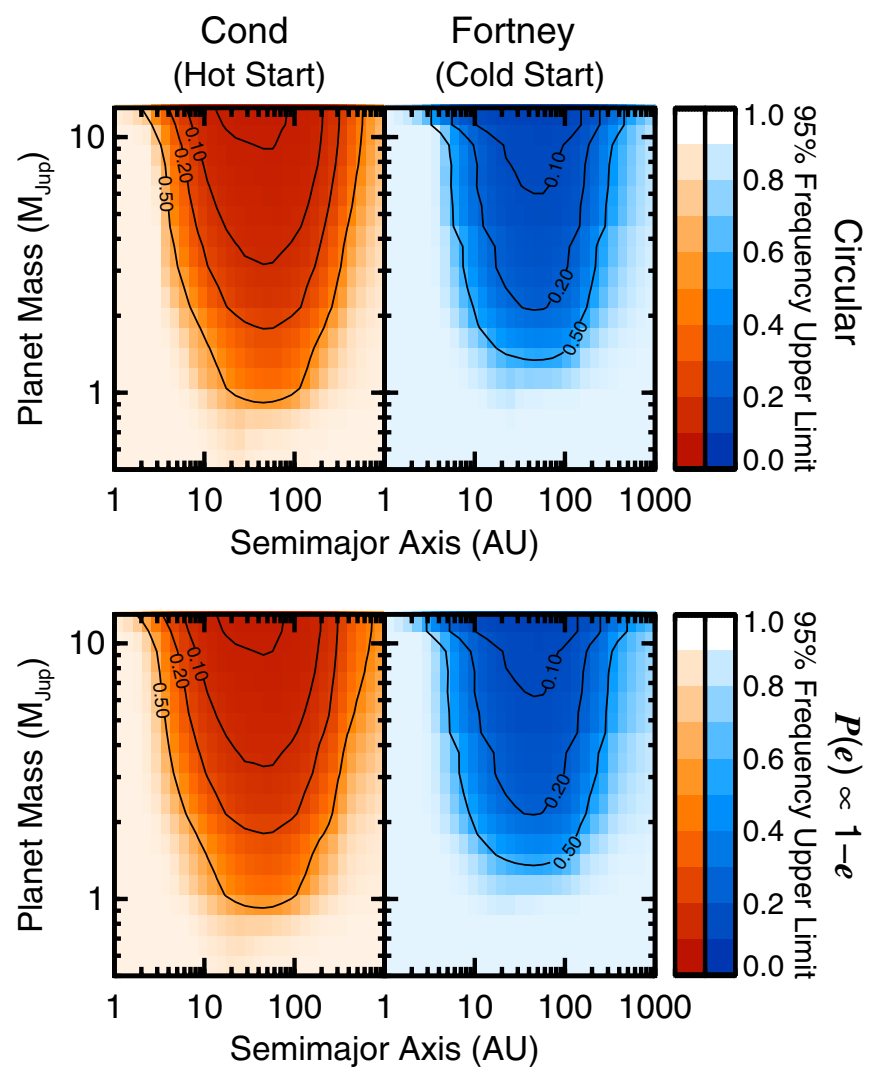

Figure 23. Upper limits on the frequency of gas giant planets. Each grid point represents the $95 \%$ confidence upper limit on the planet frequency. The strongest constraints from our survey are for massive giant planets (5-13 $M_{\mathrm{Jup}}$ ) between 10-100 AU. Contours show the 5\%,10\%, 20\%, and 50\% upper limits. 
Table 11

Giant Planet Frequency Upper Limits for a Given Planet Mass (95\% Confidence)

\begin{tabular}{|c|c|c|c|c|}
\hline $\begin{array}{l}\text { Mass } \\
\left(M_{\text {Jup }}\right)\end{array}$ & $\leqslant 5 \%$ & $\leqslant 10 \%$ & $\leqslant 20 \%$ & $\leqslant 50 \%$ \\
\hline \multicolumn{5}{|c|}{ Cond (Circular Orbits) } \\
\hline 13.0 & $13-85 \mathrm{AU}$ & $6.1-200 \mathrm{AU}$ & $3.6-320 \mathrm{AU}$ & $1.8-570 \mathrm{AU}$ \\
\hline 10.0 & 36-61 AU & 8.5-190 AU & $5.6-300 \mathrm{AU}$ & $3.3-540 \mathrm{AU}$ \\
\hline 7.0 & $\ldots$ & $11-160 \mathrm{AU}$ & $6.6-260 \mathrm{AU}$ & $3.9-470 \mathrm{AU}$ \\
\hline 5.0 & $\ldots$ & $13-140 \mathrm{AU}$ & 7.3-240 AU & $4.0-440 \mathrm{AU}$ \\
\hline 3.0 & $\ldots$ & $\ldots$ & $11-170 \mathrm{AU}$ & $5.0-330 \mathrm{AU}$ \\
\hline 2.0 & $\ldots$ & $\ldots$ & $18-120 \mathrm{AU}$ & 7.0-260 AU \\
\hline 1.0 & $\cdots$ & $\ldots$ & $\ldots$ & 18-110 AU \\
\hline \multicolumn{5}{|c|}{ Cond $(P(e) \propto 1-e)$} \\
\hline 13.0 & $13-77 \mathrm{AU}$ & 5.7-200 AU & $3.3-300 \mathrm{AU}$ & $1.6-730 \mathrm{AU}$ \\
\hline 10.0 & 36-57 AU & $8.1-180 \mathrm{AU}$ & 5.0-290 AU & $3.0-660 \mathrm{AU}$ \\
\hline 7.0 & $\ldots$ & $11-150 \mathrm{AU}$ & $6.2-260 \mathrm{AU}$ & $3.4-520 \mathrm{AU}$ \\
\hline 5.0 & $\ldots$ & $13-130 \mathrm{AU}$ & $6.7-240 \mathrm{AU}$ & $3.6-460 \mathrm{AU}$ \\
\hline 3.0 & $\ldots$ & $\ldots$ & $10-160 \mathrm{AU}$ & 4.6-320 AU \\
\hline 2.0 & $\ldots$ & $\ldots$ & $17-110 \mathrm{AU}$ & $6.3-260 \mathrm{AU}$ \\
\hline 1.0 & $\cdots$ & $\cdots$ & $\ldots$ & $17-100 \mathrm{AU}$ \\
\hline \multicolumn{5}{|c|}{ Fortney (Circular Orbits) } \\
\hline 13.0 & $\ldots$ & $10-150 \mathrm{AU}$ & 5.6-240 AU & $3.3-450 \mathrm{AU}$ \\
\hline 10.0 & $\ldots$ & 21-96 AU & 9.7-180 AU & $5.2-340 \mathrm{AU}$ \\
\hline 7.0 & $\ldots$ & $33-67$ AU & $11-160 \mathrm{AU}$ & 5.4-300 AU \\
\hline 5.0 & $\ldots$ & $\ldots$ & $12-150 \mathrm{AU}$ & 5.7-290 AU \\
\hline 3.0 & $\ldots$ & $\ldots$ & $17-110 \mathrm{AU}$ & $7.4-220 \mathrm{AU}$ \\
\hline 2.0 & $\ldots$ & $\ldots$ & 33-63 AU & 9.6-180 AU \\
\hline 1.0 & $\cdots$ & $\cdots$ & $\ldots$ & $\ldots$ \\
\hline \multicolumn{5}{|c|}{ Fortney $(P(e) \propto 1-e)$} \\
\hline 13.0 & $\ldots$ & $10-140 \mathrm{AU}$ & 5.3-250 AU & 2.9-490 AU \\
\hline 10.0 & $\ldots$ & $20-85$ AU & $9.0-180 \mathrm{AU}$ & $4.8-330 \mathrm{AU}$ \\
\hline 7.0 & $\ldots$ & $36-60 \mathrm{AU}$ & $10-150 \mathrm{AU}$ & $5.0-300 \mathrm{AU}$ \\
\hline 5.0 & $\ldots$ & $\ldots$ & $11-140 \mathrm{AU}$ & $5.2-290 \mathrm{AU}$ \\
\hline 3.0 & $\ldots$ & $\ldots$ & 17-98 AU & $6.7-220 \mathrm{AU}$ \\
\hline 2.0 & $\ldots$ & $\ldots$ & $35-54 \mathrm{AU}$ & 8.7-170 AU \\
\hline 1.0 & $\ldots$ & $\ldots$ & $\ldots$ & $\ldots$ \\
\hline
\end{tabular}

Each colored grid point reflects the $95 \%$ confidence upper limit on the planet frequency, and contours show the $50 \%, 20 \%, 10 \%$, and 5\% upper limits on planet frequency. Table 11 summarizes the semi-major axes corresponding to these upper limits for each planet mass. The best constraints are for high-mass planets between 10-100 AU, while the worst constraints are for small separations below $\sim 5 \mathrm{AU}$, large separations beyond $\sim 500 \mathrm{AU}$, and planet masses below $\sim 1 M_{\text {Jup }}$. Assuming circular orbits and hot-start cooling models, we find that fewer than $10 \%$ of single M dwarfs harbor $10 M_{\text {Jup }}\left(5 M_{\text {Jup }}\right)$ planets between 8.1-180 AU (13-130 AU). These results are insensitive to the choice of the hot-start model grid. Naturally, cold-start models produce poorer constraints; fewer than $10 \%(20 \%)$ of M dwarfs harbor $10 M_{\text {Jup }}\left(5 M_{\text {Jup }}\right)$ planets between 21-96 AU (12-150 AU) using the Fortney models.

\subsection{Giant Planet Frequency Over a Range of Planet Masses and Semi-major Axes}

Our second approach focuses on a related but slightly different question: what is the frequency of giant planets over a range of planet masses and semi-major axes? This can be addressed with our sensitivity maps and assumptions about the form of the underlying distributions of planet masses and semimajor axes. For the following analysis we adopt logarithmically flat distributions in mass from $0.5-100 M_{\mathrm{Jup}}$ and semi-major axis from 1-1000 $\mathrm{AU}: d N /(d \log a d \log m) \propto m^{\alpha} a^{\beta}$, where $\alpha=0.0$ and $\beta=0.0$. The choice of power-law representations is partly motivated (but not defined) by planet populations at smaller separations $(<10 \mathrm{AU})$, which have mass and period distributions that are well-reproduced with this functional form (e.g., Cumming et al. 2008; Howard et al. 2010). Moreover, the logarithmically flat forms are broadly consistent with the projected separation distribution and mass distribution for planets around $\mathrm{M}$ dwarfs found in microlensing surveys (Gould et al. 2010; Cassan et al. 2012). This particular case of a logarithmically flat distribution in semi-major axis corresponds to "Öpik's law" (Öpik 1924) which is a good representation of visual binaries in some circumstances (see Duchêne \& Kraus 2013 for a summary). ${ }^{12}$

In this case the number of trials for a given target is the average value over $a$ and $m$ :

$$
N_{\text {trials }}=\frac{\sum_{i=1}^{N_{\mathrm{tar}}} \sum_{j=1}^{N_{a}} \sum_{k=1}^{N_{m}} s_{i}\left(m_{k}, a_{j}\right)}{N_{a} N_{m}},
$$

where $N_{a}$ and $N_{m}$ are the number of grid points in $a$ and $m$ in the region of interest. The number of detections is zero and, once again, the binomial distribution can be used to compute an upper limit on the planet fraction at the desired level.

Table 12 summarizes the results for a various ranges of mass and semi-major axis for the Cond and Fortney models with circular and eccentric distributions. Overall our survey is most sensitive to the 10-100 $M_{\text {Jup }}$ range (Figures 24 and 25), so we would expect the tightest constraints in this region. For masses between 1 and $13 M_{\text {Jup }}$, semi-major axes between 10-100 AU, circular orbits, and a hot-start formation, $N_{\text {trials }}=26.6$, which translates into a $95 \%$ frequency upper limit of $<10.3 \%$. That is, fewer than $10.3 \%$ of $\mathrm{M}$ dwarfs harbor giant planets between 10-100 AU at the 95\% confidence level. For the cold-start models, $N_{\text {trials }}$ is reduced to 16.1 , and the upper limit is weakened to $<16.0 \%$. If we instead isolate the high-mass planet population of 5-13 $M_{\text {Jup }}, N_{\text {trials }}$ grows to 47.0 (27.7) and the upper limits tighten to $<6.0 \%(<9.9 \%)$ for the Cond (Fortney) cooling models.

As expected, exploring broader ranges of physical separation lowers $N_{\text {trials }}$ and the constraints weaken since we begin to sample regions with poor sensitivity, diluting each target's average sensitivity. For the Cond case with circular orbits between 1-10 AU, the upper limit over the entire planetary-mass range is $<51 \%$ and from $100-1000 \mathrm{AU}$ it is $<29 \%$. Likewise, for the entire 1-1000 $\mathrm{AU}, 1-13 M_{\text {Jup }}$ range, the planet frequency is $<20.0 \%$. Adding modest eccentricities tends to dilute these statistics, but overall the effect is small.

\subsection{The Frequency of Brown Dwarf Companions to M Dwarfs}

Measuring the frequency of brown dwarfs over various ranges of $a$ involves the additional step of de-projecting the observed (sky-projected) separations onto the semi-major axis plane. Like

\footnotetext{
12 Although it is a common practice in the analysis of direct imaging surveys to extrapolate power-law distributions from radial velocity-detected planets, it is not clear that extending the population of giant planets from within a few AU out to hundreds of AU is any more informative than the logarithmically uniform, scale-invariant Jeffrey's prior we have adopted. In fact, it is conceivable that giant planets are better represented by other more complex functional forms, like a power-law distribution in semi-major axis at small separations and a log-normal form at wide separations, especially if there are two modes of planet formation (e.g., Boley 2009).
} 


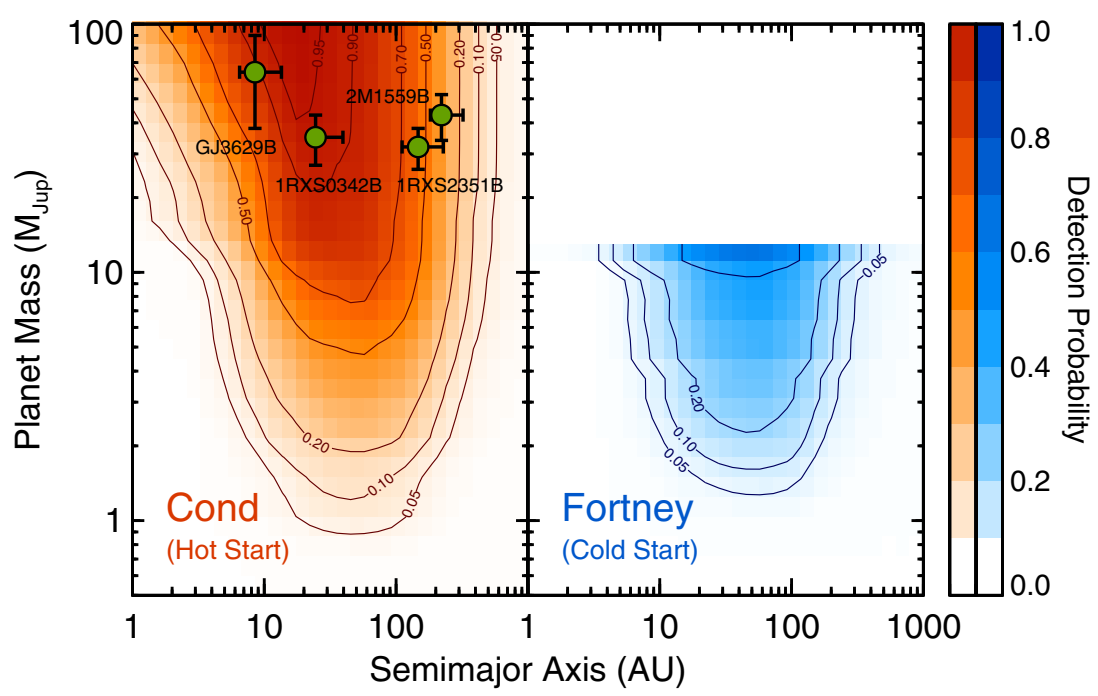

Figure 24. Survey sensitivity map showing the fraction of targets sensitive to companions between semi-major axes of $1-1000$ AU and masses of $0.5-100 \mathrm{M}$ Jup. Our four brown dwarf discoveries are shown as green circles. Contours show the 5\%,10\%, 20\%, 50\%, 90\%, and 95\% levels for the Cond (left) and Fortney (right) evolutionary models.

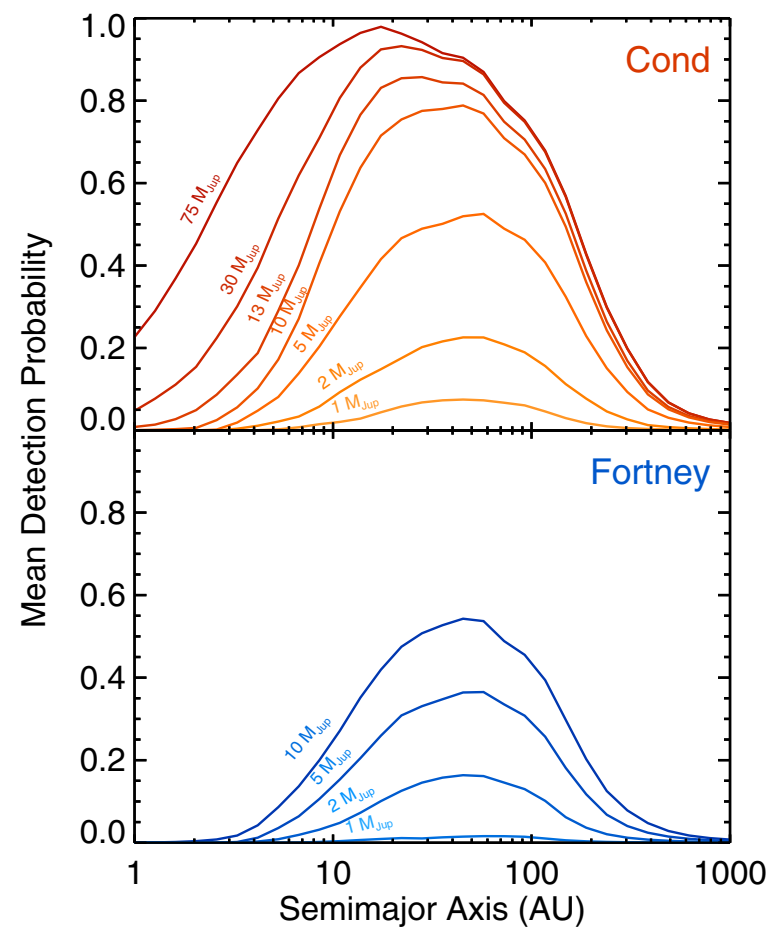

Figure 25. Survey sensitivity map showing the mean detection probability as a function of semi-major axis for varying planet masses.

the above analysis, this involves assumptions about the form of the semi-major axis distribution of substellar companions and their eccentricities (which can be defined or parameterized and freely fit). Here we adopt the same logarithmically flat distribution in $a$ assuming both circular and mildly eccentric orbits (following 1-e). Our approach is to simulate random skyprojected orbits at each step in a grid of semi-major axes, here 1-1000 AU. The number of planets at each grid point is scaled according to the power-law index used, resulting in a distribution of projected separations at each step in $a$. The cumulative distribution of projected separations over the entire range of $a$ is then used to infer the original semi-major axis distribution based on the location a companion has been observed. Uncertainties in the measured projected separation due to errors in the target's distance and angular separation measurement are incorporated in a Monte Carlo fashion.

The results of these simulations for our four brown dwarf discoveries are shown in Figure 26. In general the eccentricity distributions "smear out" to smaller physical separations, which is expected since planets can reach larger projected separations when they are on eccentric orbits. The inferred median $a$ and $68.3 \%$ confidence range about the median for GJ 3629 B, 1RXS J034231.8+121622 B, 1RXS J235133.3+ $312720 \mathrm{~B}$, and 2MASS J15594729+4403595 B is $8.5(6.5-$ 13.5) AU, $24.5(21.5,39.5) \mathrm{AU}, 145$ (105-235) AU, and 225 (182-345) AU, respectively (for circular orbits). These a priori semi-major axis distributions can then be used to compute the fraction that fall within a given range of $a$, or $N_{\text {det }}$.

If the mass of a brown dwarf companion is near the hydrogenburning limit, or if its mass uncertainty is large enough, then it is possible to overestimate the inferred substellar occurrence rate since there is a chance that object might be a low-mass star. To take this into account we weigh each of our four discoveries by the probability they are substellar using the mass distributions we derived from their age and luminosity. This mostly affects GJ $3629 \mathrm{~B}$, which has a probability of $62 \%$ of falling below the hydrogen-burning limit. The corresponding probability for 2MASS J15594729+4403595 B is $99.1 \%$, and is $100 \%$ for both 1RXS J034231.8+121622 B or 1RXS J235133.3+312720 B. Once properly weighted by their substellar probabilities, the fractional detections within some range of semi-major axis can be summed to determine $N_{\text {det }}$. Figure 26 exemplifies this for the 10-100 AU region; for circular orbits, the total contribution from each companion is $0.17,0.988,0.079$, and 0.000 , which sums to 1.23 "detections."

Applying the same analysis as in Section 6.4 to compute $N_{\text {trials }}$ between $\left\{13-75 M_{\text {Jup }}, 10-100 \mathrm{AU}\right\}$ yields 66.8 "trials," implying a substellar companion frequency of $2.8_{-1.5}^{+2.4} \%$. Similarly, we measure a frequency of $3.9_{-2.6}^{+4.8} \%$ for brown dwarfs between 1-10 AU. Over the entire range of 1-1000 AU (encompassing all four weighted detections), we find a frequency of $11.1_{-4.3}^{+5.7} \%$. Results for all permutations of $a$ are listed in Table 12. 


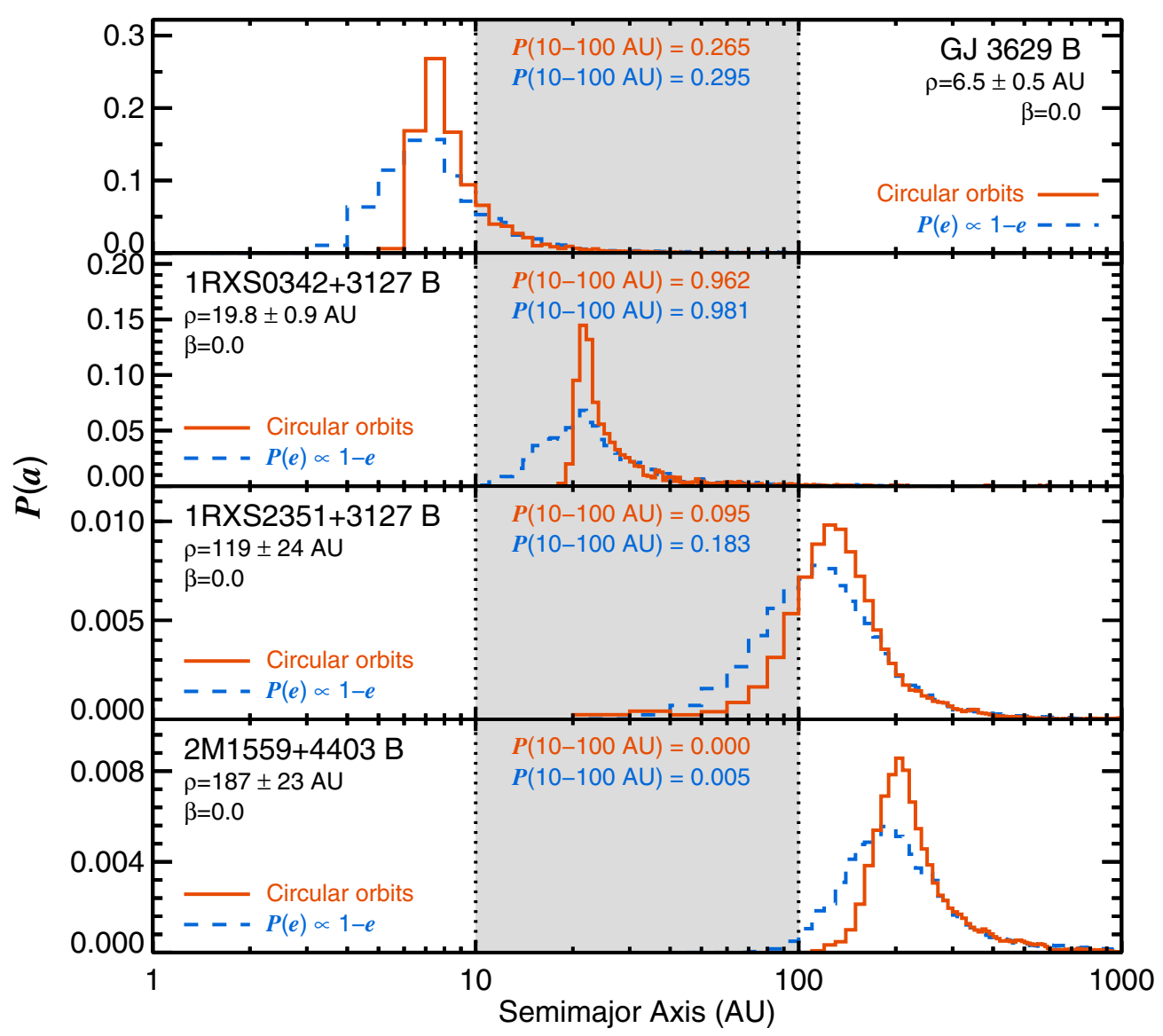

Figure 26. Semi-major axis probability distributions for the four brown dwarfs discovered in our survey based on their observed sky-projected separations. Two underlying eccentricity distributions are assumed: circular orbits and $P(e) \propto 1-e$. The latter creates a broader shape since smaller semi-major axes can reproduce the observed projected separations. The gray shaded region shows our method for computing $N_{\text {det }}$, the number of detected companions. In this example, $N_{\text {det }}$ between $10-100 \mathrm{AU}$ is the sum of all four probability distributions falling in that region (weighted by their likelihood of being substellar). Here the semi-major axis power-law index $\beta$ is flat (equal to 0.0 ) in $\log$ space.

\section{DISCUSSION}

The well-established correlation between stellar host mass and giant planet frequency offers one of the strongest cases for core accretion at small separations $(\lesssim 2.5 \mathrm{AU}$; Johnson et al. 2007,2010 ). Since orbital period scales as $M_{*}^{-1 / 2}$, the timescale associated with planetesimal coagulation (a few Myr) is faster for high-mass stars so more cores are able to form and accrete gaseous envelopes before protoplanetary disks disperse (e.g., Laughlin et al. 2004; Kennedy \& Kenyon 2008). In addition, there is now ample observational evidence that protoplanetary disk masses scale with stellar host mass, resulting in increased raw material for giant planet formation around high-mass stars compared to low-mass stars (Andrews et al. 2013; Mohanty et al. 2013).

Much less is known about the dependence of wide-separation ( $>10 \mathrm{AU}$ ) giant planet frequency on stellar host mass. Like core accretion at small separations, disk instability predicts a positive trend with primary mass assuming protoplanetary disk masses scale with protostellar mass (Boss 2011). A total of seven gasgiant planets have been directly imaged to date around three high-mass $\left(1.2-1.9 M_{\odot}\right)$ young A-type stars and one $\mathrm{G}$ star (HR 8799, $\beta$ Pic, HD 95086, GJ 504; Marois et al. 2008, 2010b; Rameau et al. 2013b; Kuzuhara et al. 2013). Around low-mass stars, companions near the deuterium-burning limit $\left(\approx 13 M_{\text {Jup }}\right)$ have been found at close separations within 100 AU (e.g.,
2MASS J01033563-5515561 C, Delorme et al. 2013; 2MASS J01225093-2439505 B, Bowler et al. 2013), and a growing population of planetary-mass objects on extreme orbits beyond 100 AU has been identified (e.g., GU Psc b, Naud et al. 2014). However, no companions below $10 M_{\text {Jup }}$ have been imaged at $<100 \mathrm{AU}$ around stars between $0.1-1.0 M_{\odot},{ }^{13}$ perhaps pointing to a correlation between stellar mass and giant planet occurrence rate (Crepp \& Johnson 2011).

On the other hand, this apparent trend can also be explained by a selection bias since nearly all large direct imaging planet searches are focusing on high-mass stars. For example, the NICI Planet-Finding Campaign (Liu et al. 2010a), Gemini Planet Imager Exoplanet Survey (Macintosh et al. 2014), SEEDS (Tamura et al. 2006), LBTI Exozodi Exoplanet Common Hunt (Skemer et al. 2014), and the International Deep Planet Search (Vigan et al. 2012) concentrate on AFGK stars $\left(\approx 0.6-2 M_{\odot}\right)$, so a paucity of imaged planets around low-mass stars is not surprising.

The only way to test whether giant planet frequency correlates with stellar host mass is to compare the statistical properties of long-period planets in different stellar mass regimes. The largest imaging program targeting high-mass stars is the NICI

\footnotetext{
13 Interestingly, several planetary-mass companions are known around brown dwarfs (Chauvin et al. 2004; Todorov et al. 2010; Liu et al. 2012; Han et al. 2013), indicating an alternative formation mechanism of planetary-mass companions around very low host masses.
} 
Table 12

The Frequency of Brown Dwarfs and Giant Planets Around Low-mass Stars

\begin{tabular}{|c|c|c|c|c|c|}
\hline $\begin{array}{l}\text { Mass } \\
\left(M_{\text {Jup }}\right)\end{array}$ & $\begin{array}{c}a \\
(\mathrm{AU})\end{array}$ & $\begin{array}{l}\text { Eccentricity } \\
\text { Distribution }\end{array}$ & $N_{\text {det }}$ & $N_{\text {trials }}$ & Frequency \\
\hline \multicolumn{6}{|c|}{ Brown Dwarf Frequency (Cond) } \\
\hline $13-75$ & $1-10$ & Circ & 0.41 & 26.6 & $4_{-3}^{+5} \%$ \\
\hline $13-75$ & $1-10$ & $1-e$ & 0.41 & 28.0 & $4_{-2}^{+4} \%$ \\
\hline $13-75$ & $1-20$ & Circ & 0.59 & 36.0 & $3_{-2}^{+4} \%$ \\
\hline $13-75$ & $1-20$ & $1-e$ & 0.59 & 37.0 & $3_{-2}^{+4} \%$ \\
\hline $13-75$ & $1-100$ & Circ & 1.68 & 46.7 & $5_{-3}^{+4} \%$ \\
\hline $13-75$ & $1-100$ & $1-e$ & 1.68 & 47.0 & $5_{-3}^{+4} \%$ \\
\hline $13-75$ & $1-1000$ & Circ & 3.61 & 37.4 & $11_{-4}^{+6} \%$ \\
\hline $13-75$ & $1-1000$ & $1-e$ & 3.61 & 37.4 & $11_{-4}^{+6} \%$ \\
\hline $13-75$ & $10-100$ & Circ & 1.23 & 66.8 & $3_{-2}^{+2} \%$ \\
\hline $13-75$ & $10-100$ & $1-e$ & 1.23 & 66.0 & $3_{-2}^{+3} \%$ \\
\hline $13-75$ & $10-200$ & Circ & 2.18 & 61.4 & $5_{-2}^{+3} \%$ \\
\hline $13-75$ & $10-200$ & $1-e$ & 2.18 & 60.1 & $5_{-2}^{+3} \%$ \\
\hline $13-75$ & $10-1000$ & Circ & 3.17 & 42.7 & $9_{-4}^{+5} \%$ \\
\hline $13-75$ & $10-1000$ & $1-e$ & 3.17 & 42.0 & $9_{-4}^{+5} \%$ \\
\hline $13-75$ & $100-1000$ & Circ & 1.88 & 18.7 & $13_{-6}^{+9} \%$ \\
\hline $13-75$ & $100-1000$ & $1-e$ & 1.88 & 18.0 & $13_{-6}^{+9} \%$ \\
\hline \multicolumn{6}{|c|}{ Giant Planet Frequency (95\% Confidence; Cond) } \\
\hline $1-13$ & $1-10$ & Circ & 0.00 & 3.1 & $<51 \%$ \\
\hline $1-13$ & $1-10$ & $1-e$ & 0.00 & 3.7 & $<47 \%$ \\
\hline $1-13$ & $1-20$ & Circ & 0.00 & 7.3 & $<30 \%$ \\
\hline $1-13$ & $1-20$ & $1-e$ & 0.00 & 7.8 & $<29 \%$ \\
\hline $1-13$ & $1-100$ & Circ & 0.00 & 14.9 & $<17 \%$ \\
\hline $1-13$ & $1-100$ & $1-e$ & 0.00 & 15.1 & $<17 \%$ \\
\hline $1-13$ & $1-1000$ & Circ & 0.00 & 12.4 & $<20 \%$ \\
\hline $1-13$ & $1-1000$ & $1-e$ & 0.00 & 12.5 & $<20 \%$ \\
\hline $1-13$ & $10-100$ & Circ & 0.00 & 26.6 & $<10 \%$ \\
\hline $1-13$ & $10-100$ & $1-e$ & 0.00 & 26.4 & $<10 \%$ \\
\hline $1-13$ & $10-200$ & Circ & 0.00 & 24.8 & $<11 \%$ \\
\hline $1-13$ & $10-200$ & $1-e$ & 0.00 & 24.3 & $<11 \%$ \\
\hline $1-13$ & $10-1000$ & Circ & 0.00 & 17.2 & $<15 \%$ \\
\hline $1-13$ & $10-1000$ & $1-e$ & 0.00 & 16.9 & $<15 \%$ \\
\hline $1-13$ & $100-1000$ & Circ & 0.00 & 7.7 & $<29 \%$ \\
\hline $1-13$ & $100-1000$ & $1-e$ & 0.00 & 7.4 & $<30 \%$ \\
\hline $5-13$ & 1-10 & Circ & 0.00 & 6.2 & $<34 \%$ \\
\hline $5-13$ & $1-10$ & $1-e$ & 0.00 & 7.3 & $<30 \%$ \\
\hline $5-13$ & $1-20$ & Circ & 0.00 & 13.9 & $<18 \%$ \\
\hline $5-13$ & $1-20$ & $1-e$ & 0.00 & 14.9 & $<17 \%$ \\
\hline $5-13$ & $1-100$ & Circ & 0.00 & 26.6 & $<10 \%$ \\
\hline $5-13$ & $1-100$ & $1-e$ & 0.00 & 26.9 & $<10 \%$ \\
\hline $5-13$ & $1-1000$ & Circ & 0.00 & 22.5 & $<12 \%$ \\
\hline $5-13$ & $1-1000$ & $1-e$ & 0.00 & 22.6 & $<12 \%$ \\
\hline $5-13$ & $10-100$ & Circ & 0.00 & 47.0 & $<6.0 \%$ \\
\hline $5-13$ & $10-100$ & $1-e$ & 0.00 & 46.6 & $<6.1 \%$ \\
\hline $5-13$ & 10-200 & Circ & 0.00 & 43.9 & $<6.4 \%$ \\
\hline $5-13$ & 10-200 & $1-e$ & 0.00 & 43.0 & $<6.6 \%$ \\
\hline $5-13$ & $10-1000$ & Circ & 0.00 & 30.6 & $<9.0 \%$ \\
\hline $5-13$ & $10-1000$ & $1-e$ & 0.00 & 30.2 & $<9.1 \%$ \\
\hline $5-13$ & $100-1000$ & Circ & 0.00 & 14.3 & $<18 \%$ \\
\hline $5-13$ & $100-1000$ & $1-e$ & 0.00 & 13.8 & $<18 \%$ \\
\hline \multicolumn{6}{|c|}{ Giant Planet Frequency (95\% Confidence; Fortney) } \\
\hline $1-13$ & $1-10$ & Circ & 0.00 & 1.4 & $<71 \%$ \\
\hline $1-13$ & $1-10$ & $1-e$ & 0.00 & 1.7 & $<67 \%$ \\
\hline $1-13$ & $1-20$ & Circ & 0.00 & 3.7 & $<47 \%$ \\
\hline $1-13$ & $1-20$ & $1-e$ & 0.00 & 4.1 & $<45 \%$ \\
\hline $1-13$ & $1-100$ & Circ & 0.00 & 8.7 & $<26 \%$ \\
\hline $1-13$ & $1-100$ & $1-e$ & 0.00 & 8.8 & $<26 \%$ \\
\hline $1-13$ & $1-1000$ & Circ & 0.00 & 7.1 & $<31 \%$ \\
\hline $1-13$ & $1-1000$ & $1-e$ & 0.00 & 7.1 & $<31 \%$ \\
\hline
\end{tabular}

Table 12

(Continued)

\begin{tabular}{lccccc}
\hline \hline $\begin{array}{l}\text { Mass } \\
\left(M_{\text {Jup }}\right)\end{array}$ & $\begin{array}{c}a \\
\mathrm{AU})\end{array}$ & $\begin{array}{c}\text { Eccentricity } \\
\text { Distribution }\end{array}$ & $N_{\text {det }}$ & $N_{\text {trials }}$ & Frequency \\
\hline $1-13$ & $10-100$ & Circ & 0.00 & 16.1 & $<16 \%$ \\
$1-13$ & $10-100$ & $1-e$ & 0.00 & 15.9 & $<16 \%$ \\
$1-13$ & $10-200$ & Circ & 0.00 & 14.6 & $<17 \%$ \\
$1-13$ & $10-200$ & $1-e$ & 0.00 & 14.3 & $<18 \%$ \\
$1-13$ & $10-1000$ & Circ & 0.00 & 9.9 & $<24 \%$ \\
$1-13$ & $10-1000$ & $1-e$ & 0.00 & 9.8 & $<24 \%$ \\
$1-13$ & $100-1000$ & Circ & 0.00 & 3.8 & $<46 \%$ \\
$1-13$ & $100-1000$ & $1-e$ & 0.00 & 3.7 & $<47 \%$ \\
$5-13$ & $1-10$ & Circ & 0.00 & 2.8 & $<54 \%$ \\
$5-13$ & $1-10$ & $1-e$ & 0.00 & 3.3 & $<50 \%$ \\
$5-13$ & $1-20$ & Circ & 0.00 & 7.0 & $<31 \%$ \\
$5-13$ & $1-20$ & $1-e$ & 0.00 & 7.6 & $<30 \%$ \\
$5-13$ & $1-100$ & Circ & 0.00 & 15.3 & $<17 \%$ \\
$5-13$ & $1-100$ & $1-e$ & 0.00 & 15.4 & $<17 \%$ \\
$5-13$ & $1-1000$ & Circ & 0.00 & 12.5 & $<20 \%$ \\
$5-13$ & $1-1000$ & $1-e$ & 0.00 & 12.6 & $<20 \%$ \\
$5-13$ & $10-100$ & Circ & 0.00 & 27.7 & $<9.9 \%$ \\
$5-13$ & $10-100$ & $1-e$ & 0.00 & 27.5 & $<10 \%$ \\
$5-13$ & $10-200$ & Circ & 0.00 & 25.4 & $<11 \%$ \\
$5-13$ & $10-200$ & $1-e$ & 0.00 & 24.9 & $<11 \%$ \\
$5-13$ & $10-1000$ & Circ & 0.00 & 17.4 & $<15 \%$ \\
$5-13$ & $10-1000$ & $1-e$ & 0.00 & 17.7 & $<15 \%$ \\
$5-13$ & $100-1000$ & Circ & 0.00 & 7.1 & $<31 \%$ \\
$5-13$ & $100-1000$ & $1-e$ & 0.00 & 6.8 & $<32 \%$ \\
\hline & & & & &
\end{tabular}

Planet-Finding Campaign (Liu 2010a)Liu Wahhaj). From their subsample of 70 young B- and A-type stars, Nielsen et al. (2013) find that fewer than $20 \%$ of $1.5-2.5 M_{\odot}$ stars harbor $>4 M_{\text {Jup }}$ planets between 59-460 AU. Other smaller surveys have mostly resulted in upper limits or, in some cases, weak constraints if the HR 8799 and/or $\beta$ Pic systems are included (Ehrenreich et al. 2010; Janson et al. 2011; Rameau et al. 2013a). For example, Vigan et al. (2012) targeted 38 A stars and 4 F stars and arrived at a frequency of $4.3_{-1.3}^{+9.1} \%$ when $\beta$ Pic $\mathrm{b}$ is excluded (a priori knowledge of its existence can strongly bias the way the observations are conducted).

Several large $(N>50)$ direct imaging surveys have focused on young Sun-like stars. The analysis of 100 FGK stars by Nielsen \& Close (2010), which combined the surveys of Masciadri et al. (2005), Biller et al. (2007), and Lafrenière et al. (2007a), is the largest study of wide-period planets around $0.6-1.2 M_{\odot}$ host stars to date. No planets were detected, yielding an upper limit of $<20 \%$ (at the $95 \%$ confidence level) for the frequency of $>4 M_{\text {Jup }}$ planets between $\approx 40-470 \mathrm{AU}$. More recently, Chauvin et al. (2014) measured similar constraints of $<15 \%$ for $>5 M_{\text {Jup }}$ planets between 100-300 AU in their sample of 51 Sun-like stars.

We find an upper limit of $<6.0 \%$ in this survey of 78 single young $\mathrm{M}$ dwarfs, which is by far the most substantial program to date in the low-mass regime. Taken together with similarly large surveys targeting A and FGK stars, there is currently no statistical evidence for a dependency of giant planet frequency with stellar host mass. In the future, larger sample sizes will be needed to distinguish between small differences in the relative occurrence rates of long-period giant planets around A stars and M dwarfs. 


\subsection{A Constant Substellar Companion Fraction with Host Mass}

The relative occurrence rates of brown dwarf companions as a function of stellar host mass also provides clues about their formation. Large-scale hydrodynamical simulations of fragmenting molecular clouds by Bate $(2009,2012)$ produced brown dwarf companion frequencies with no discernible dependency on the primary host star mass. This seems to be consistent with observations: Vigan et al. (2012) find a frequency of $2.8_{-0.9}^{+6.0} \%$ between 5-320 AU for massive A and F stars, Metchev \& Hillenbrand (2009) find a frequency of $3.2_{-2.7}^{+3.1} \%$ ( $2 \sigma$ limits) between 28-1590 AU around FGK stars, and we infer a rate of $2.8_{-1.5}^{+2.4} \%$ $\left(4.5_{-2.1}^{+3.1} \%\right)$ between $10-100 \mathrm{AU}(10-200 \mathrm{AU})$ for M dwarfs. Although the ranges of semi-major axes being considered are different in these studies, they all point to comparable rates of a few percent across all separations.

Metchev \& Hillenbrand (2009) compared all published direct imaging searches for brown dwarf companions as of 2009 and found a tentative trend between the frequency of brown dwarf companions and both stellar host mass and separation. Surveys targeting low-mass stars $\left(\approx 0.2-0.6 M_{\odot}\right)$ at small separations $(\lesssim 150 \mathrm{AU})$ found a paucity of brown dwarfs compared to those focusing on more massive stars $\left(\gtrsim 0.7 M_{\odot}\right)$ and wide separations ( $\gtrsim 150 \mathrm{AU})$. However, our results do not support this correlation; our brown dwarf companion frequency of a few percent is similar to the higher-mass, wide-separation surveys. As emphasized by Metchev \& Hillenbrand, most of these previous surveys did not correct for incompleteness in their observations, so the inferred substellar frequencies should be treated with caution. On the other hand, our deep observations probe the entire substellar regime and we correct for incompleteness in the regions in which we are not sensitive. Incidentally, two additional brown dwarfs were previously known at separations of $\approx 350-400$ AU around single stars in our sample (G 196-3 and NLTT 22741; Rebolo et al. 1998; Reid $\&$ Walkowicz 2006). Neither were detected in our data so they were not included in our statistical results, but together they imply that at least six out of 78 single $M$ dwarfs in our sample host substellar companions, a rate much higher than inferred from previous, less sensitive surveys targeting low-mass stars listed in Metchev \& Hillenbrand. Our results are supported by the HST multiplicity survey by Dieterich et al. (2012), which found a multiplicity rate of $2.3_{-0.7}^{+5.0} \%$ for L0-T9 companions to field $\mathrm{M}$ dwarfs. ${ }^{14}$

An ongoing debate over whether gas giants can form via direct gravitational collapse of a massive protoplanetary disk has consumed much of the discussion about planet formation for the past decade, especially after the discovery of the HR 8799 planets (e.g., Boss 2007; Durisen et al. 2007; Boley 2009; Dodson-Robinson et al. 2009; Nero \& Bjorkman 2009; Kratter et al. 2010). Simulations show that protoplanetary disks can collapse when conditions are both cool enough and disk surface densities are high enough. The region between a few tens to a few hundreds of AU occupies this "sweet spot" and is the most likely place for giant planets to form from this mechanism (e.g., Stamatellos \& Whitworth 2009; Vorobyov \& Basu 2010; Boss 2011). Our constraints on the frequency of giant planets in this region for the most common type of star imply that, overall,

\footnotetext{
14 Note that old low-mass stars have effective temperatures reaching early L spectral types, so this frequency is slightly different from the substellar companion fraction.
}

disk instability is not an efficient mechanism for producing gas giants around low-mass stars.

\section{SUMMARY AND CONCLUSIONS}

We have carried out a deep direct imaging search for giant planets around nearby $(\lesssim 40 \mathrm{pc})$ young $(\lesssim 300 \mathrm{Myr})$ lowmass stars with Keck and Subaru. Out of 122 targets, 44 are resolved into close visual binaries with separations ranging from $\approx 0$ '. $05-2^{\prime \prime} ; 27$ of these are new or spatially resolved for the first time. Because known binaries were removed prior to the start of this survey, we infer a minimum stellar companion frequency of $>35.4 \pm 4.3 \%$ within $100 \mathrm{AU} .38 \%$ of our sample are confirmed or likely members of YMGs spanning ages of 8-620 Myr and 57\% of our targets have measured parallaxes. Below we summarize results of our deep imaging search for planets around the 78 single $\mathrm{M}$ dwarfs in our sample:

1. Four comoving brown dwarfs with masses between 30-70 $M_{\text {Jup }}$ and projected separations of 6-190 AU were discovered in our survey: 1RXS J235133.3+312720 B (Bowler et al. 2012b), GJ 3629 B (Bowler et al. 2012a), 1RXS J034231.8+121622 B, and 2MASS J15594729+4403595 B. 1RXS J235133.3+312720 is likely a member of the $\approx 120$ Myr AB Dor moving group.

2. Taking into account our detection limits, we measure a brown dwarf companion fraction of $2.8_{-1.5}^{+2.4} \%\left(4.5_{-2.1}^{+3.1} \%\right)$ between 10-100 AU (10-200 AU) around single M dwarfs. These results are consistent with the brown dwarf occurrence rate found around high- and intermediate-mass primaries, which is also in general agreement with hydrodynamical simulations of turbulent fragmentation by Bate (2009).

3. No planets were confirmed in our survey. Among 102 candidates detected around 38 single stars in our deep imaging, 60 are shown to be stationary background stars. The status of the remaining 42 candidates with only a single epoch of astrometry is unclear, but only 8 of these are located at projected separations less than $100 \mathrm{AU}$.

4. Our null detection of planets implies that $<10.3 \%(<6.0 \%)$ of single $\mathrm{M}$ dwarfs harbor 1-13 $M_{\text {Jup }}\left(5-13 M_{\text {Jup }}\right)$ planets between 10-100 AU assuming hot-start evolutionary models and logarithmically uniform distributions in planet mass and semi-major axis.

5. The dearth of massive planets at tens to hundreds of AU around the most common type of star in our galaxy implies that, overall, disk instability is not a common mechanism of giant plant formation.

6. Finally, comparing the largest direct imaging planet searches in three mass regimes (A, FGK, and M stars), there is currently no statistical evidence for a correlation between stellar host mass and giant planet frequency at large separations (>10 AU).

In the future, much larger samples of several hundred stars in each stellar mass bin will be needed to discriminate differences in the relative frequencies of giant planets at $\gtrsim 10 \mathrm{AU}$. We caution that for large homogeneous analyses incorporating our contrast curves and those of any other surveys, not all planet candidates have been rejected as background stars and so targets and contrast curves must be carefully selected on a case-by-case basis. Ultimately, large statistical comparisons with the current generation of instruments on 8-10 meter class telescopes will set the stage-and statistical baseline-for the next generation 
of 30 meter telescopes to image true Jupiter analogs in the 3-10 AU region.

We are grateful to our anonymous referee for helpful comments, Katelyn Allers for the low gravity spectral templates used in this work, Adam Kraus and Trent Dupuy for assistance with some of the observations, John Johnson for constructive comments on this paper, and Kimberly Aller for measuring gravity indices. It is a pleasure to thank the telescope operators and support astronomers Jun Hashimoto, Alan Hatakeyama, Ryo Kandori, Tomoyuki Kudo, Nobahiko Kusakabe, and Joshua Williams at Subaru Telescope and Joel Aycock, Randy Campbell, Al Conrad, Heather Hershley, Marc Kassis, Jim Lyke, Jason McIlroy, Barbara Schaefer, Terry Stickel, Hien Tran, and Cynthia Wilburn at Keck Observatory for their support with the observations. B.P.B. and M.C.L. have been supported by NASA grant NNX11AC31G and NSF grant AST09-09222. We utilized data products from the Two Micron All Sky Survey, which is a joint project of the University of Massachusetts and the Infrared Processing and Analysis Center/California Institute of Technology, funded by the National Aeronautics and Space Administration and the National Science Foundation. NASA's Astrophysics Data System Bibliographic Services together with the VizieR catalogue access tool and SIMBAD database operated at CDS, Strasbourg, France, were invaluable resources for this work. This research has made use of the Washington Double Star Catalog maintained at the U.S. Naval Observatory. Finally, mahalo nui loa to the kama'āina of Hawai'i for their support of Keck and the Mauna Kea observatories. We are grateful to conduct observations from this mountain.

Facilities: Keck:II (NIRC2), Subaru (HiCIAO), Keck:II (OSIRIS), IRTF (SpeX)

\section{APPENDIX A}

\section{HICIAO DISTORTION CORRECTION}

The HiCIAO optical distortion, plate scale, and orientation were measured using $H$-band images of the globular cluster M5 obtained on the nights of 2011 January 27 UT and 2012 May 10 UT. We targeted $\mathrm{a} \approx 20^{\prime \prime} \times 20^{\prime \prime}$ region near the center of the cluster covering the same dense stellar field as in Cameron et al. (2009). The HiCIAO camera lens was changed in 2011 April, so we generated two distortion maps: one for our 2011 January observing run, and one for our 2011 December/2012 January and 2012 May runs. NIRC2 images of the same field obtained on 2006 February 7 UT using the wide camera (A. Kraus 2013, private communication) were used as an absolute reference frame. We first corrected the NIRC2 optical distortions using the solution created by B. Cameron (2007, private communication). Stars were then identified in the images from both instruments with the DAOPHOT photometry package (Stetson 1987). Finally, the AMOEBA downhill simplex algorithm was used to fit for relative $x / y$ offsets (in pixel coordinates) between the two systems, a relative magnification scale for HiCIAO, an overall rotation of HiCIAO, and 18 coefficients comprising a 2D, thirdorder polynomial fit following Anderson \& King (2003). A total of 297 and 344 stars are used for our 2011 and 2012 calibration measurements, respectively.

The best-fit distortion solutions are shown in Figures 27 and 28. The upgraded camera lens created a significant qualitative difference in the optical distortion, with most of the optical aberrations occurring in the $y$-direction along the detector columns with the new lens in place. The uncorrected optical dis- tortion produces significant positional offsets of up to 30 pixels near the edges of the detector at both epochs. After applying our solution, the average total residual displacement between the HiCIAO and NIRC2 positions is 0.8 pixel and 1.2 pixel for the 2011 and 2012 calibration data sets, respectively, showing little dependence on spatial position across the entire $2048 \times$ 2048 pixel array. We therefore adopt 1 pixel as a typical systematic positional uncertainty caused by optical distortions $\left(\sigma_{d}\right)$ for our HiCIAO observations. The best-fit solutions give magnification scales of 4.103 and 4.100 times smaller than the NIRC2 wide camera for the 2011 and 2012 data. We also solved for HiCIAO distortion solutions at each epoch using the same field and instrument setup, except with the coronagraph slide in place to test its influence on the astrometry. The results are virtually identical to the solutions without the coronagraph in place, giving magnification scales of 4.104 and 4.101 at each epoch. Because of these similarities, we assume identical magnification factors of $4.102 \pm 0.002$. Based on the NIRC2 wide camera plate scale of $39.884 \pm 0.039$ mas pixel ${ }^{-1}$ measured by Pravdo et al. (2006), this implies a HiCIAO $H$-band plate scale of $9.723 \pm$ 0.011 mas pixel $^{-1}$. The HiCIAO detector appears to be aligned very closely with celestial north; the best-fit solutions imply rotations of $-0.01(-0.09)$ and $+0.03(+0.03)$ for the 2011 and 2012 data sets without (with) the coronagraph (positive is east from north). We conservatively adopt a detector orientation of $0.0 \pm 0.1$.

Our observations of the 5".6 pair 2MASS J15594729+ $4403595 \mathrm{AB}$ in the $Y, J, H$, and $K_{S}$ filters at the same position on the detector show that the HiCIAO plate scale varies significantly with wavelength. The separation in $H$ band is $580.8 \pm$ 1.4 pixel, or $5647 \pm 15$ mas using our plate scale measurement, which is in excellent agreement with the value of $5638 \pm 0$ '.004 measured by Janson et al. (2012, no orbital motion is expected given the system's $\sim 190$ AU separation). On the other hand, the separations in $Y, J$, and $K_{S}$ bands are $575.5 \pm 1.4$ pixel, $579.2 \pm$ 1.4 pixel, and $583.7 \pm 1.4$ pixel, respectively, implying plate scales of $9.81 \pm 0.04$ mas pixel ${ }^{-1}, 9.75 \pm 0.04$ mas pixel ${ }^{-1}$, $9.67 \pm 0.03$ mas pixel $^{-1}$. We adopt these wavelength-dependent plate scales for our astrometry. On the other hand, the P.A.s are consistent within 0.01 , so we do not make any corrections to that.

\section{APPENDIX B}

\section{NIRC2 600 MAS CORONAGRAPH ATTENUATION}

NIRC2 has several circular, partly transmissive occulting spots located on a clear slide in its first focal plane. We made use the 600 mas diameter spot for our survey. To calibrate the transmission, we observed the young, 2".9 separation M1.5+M4.0 binary NLTT 32659 (Shkolnik et al. 2009) on 2012 May 21 UT with and without the primary under the spot. The observations and resulting flux ratios are listed in Table 13. Aperture photometry using an extraction radius of 5 pixel yields a transmission of $7.51 \pm 0.14 \mathrm{mag}(0.099 \pm 0.013 \%)$ in $H$ and $6.65 \pm$ $0.10 \mathrm{mag}(0.22 \pm 0.02 \%)$ in $K_{S}$. Incidentally, our P.A. and separation measurements are in excellent agreement with Shkolnik et al. (2012).

\section{APPENDIX C}

\section{NOTES ON INDIVIDUAL OBJECTS}

GJ 3030 AB (2MASS J00215781+4912379). GJ 3030 AB was first identified as a visual binary in the Washington 

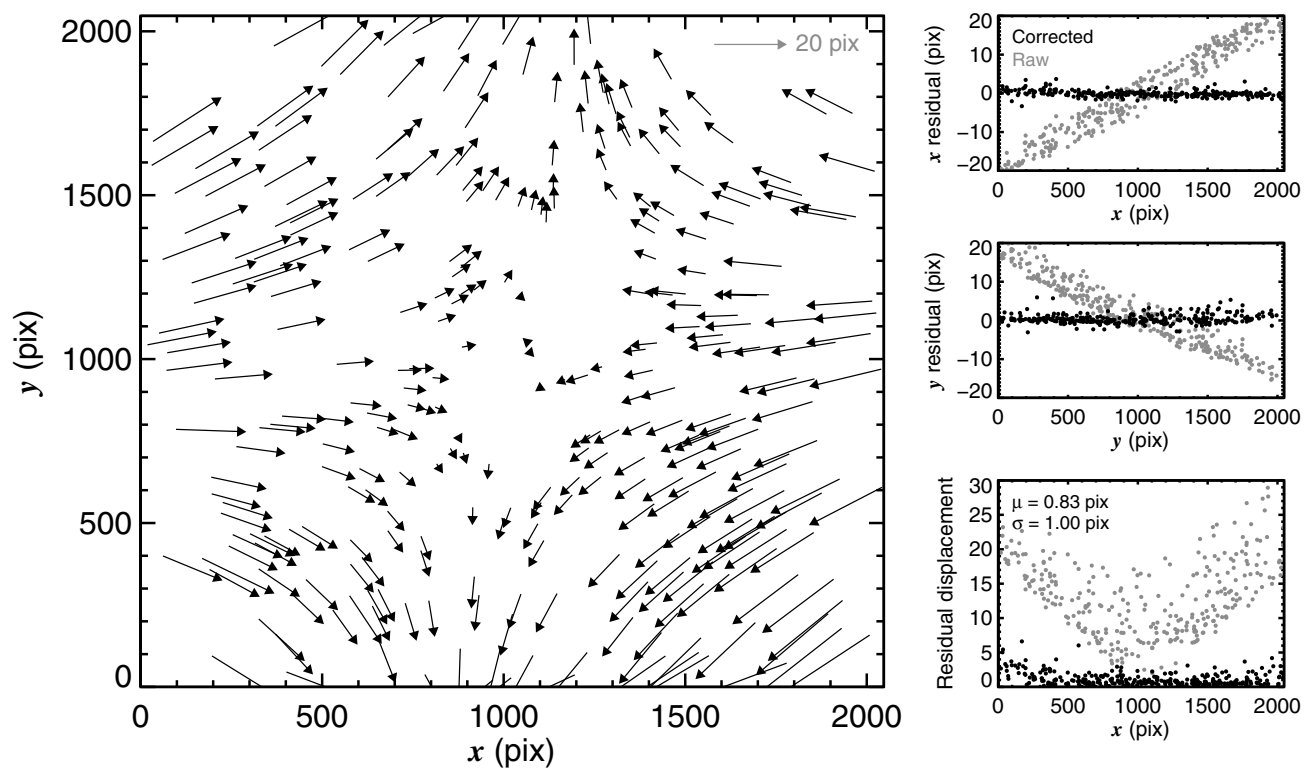

Figure 27. Our HiCIAO distortion solution from 2011 January. Arrow bases and heads indicate the measured and corrected stellar positions, respectively, in our images of M5 after applying third-order polynomial polynomial fit in $x$ and $y$. For visual purposes all arrow lengths have been increased by a factor of 10 . The average residual displacement after correction is 0.8 pixel ( 7.8 mas in $H$ band).
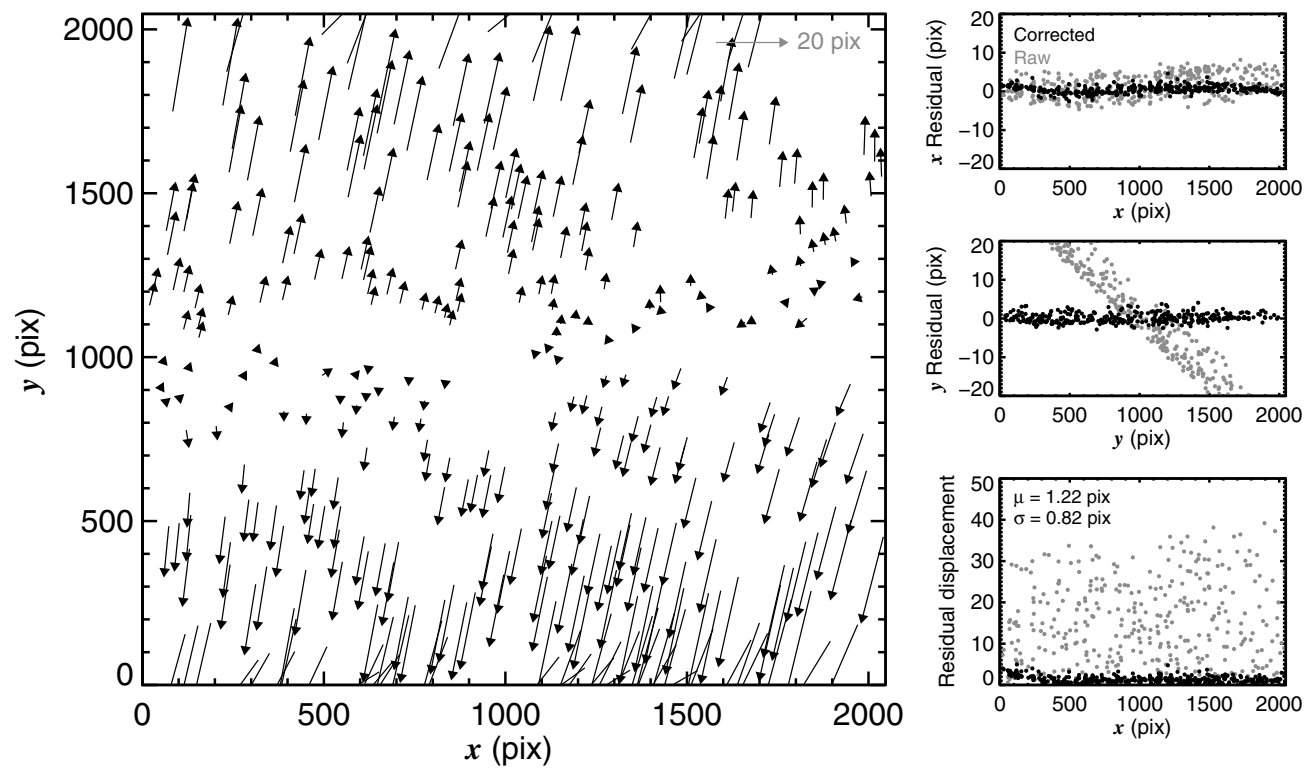

Figure 28. Our HiCIAO distortion solution from 2012 May. In 2011 April the HiCIAO camera lens was replaced, creating a substantially different distortion map compared to the 2011 January one shown in Figure 27. After correction, the typical residual displacement is 1.2 pixel ( 11.7 mas in $H$ band). Arrow lengths have been increased by a factor of 10 for visual purposes.

Table 13

NIRC2 Coronagraph Calibration Measurements of NLTT 32659 AB

\begin{tabular}{|c|c|c|c|c|c|c|c|c|}
\hline $\begin{array}{l}\text { Date } \\
\text { (UT) }\end{array}$ & Coronagraph & Filter & $\begin{array}{c}N \times \text { Coadds } \times \\
\text { Exp. Time }(\mathrm{s})\end{array}$ & $\begin{array}{l}\text { FWHM } \\
\text { (mas) }\end{array}$ & Strehl & $\begin{array}{l}\text { Separation } \\
(")\end{array}$ & $\begin{array}{l}\text { P.A. } \\
\left({ }^{\circ}\right)\end{array}$ & $\Delta$ mag \\
\hline 2012 May 21 & None & $K_{S}$ & $28 \times 10 \times 0.15$ & $61 \pm 7$ & $0.16 \pm 0.08$ & $2.899 \pm 0.002$ & $88.54 \pm 0.03$ & $2.03 \pm 0.04$ \\
\hline 2012 May 21 & 600 mas & $K_{S}$ & $13 \times 1 \times 3.0$ & $62 \pm 4$ & $0.11 \pm 0.03$ & $2.898 \pm 0.002$ & $88.64 \pm 0.03$ & $4.60 \pm 0.09$ \\
\hline 2012 May 21 & None & $H$ & $17 \times 1 \times 0.15$ & $50 \pm 4$ & $0.10 \pm 0.04$ & $2.897 \pm 0.003$ & $88.59 \pm 0.06$ & $2.18 \pm 0.07$ \\
\hline 2012 May 21 & 600 mas & $H$ & $12 \times 1 \times 2.5$ & $53 \pm 4$ & $0.08 \pm 0.02$ & $2.902 \pm 0.004$ & $88.59 \pm 0.07$ & $5.30 \pm 0.11$ \\
\hline
\end{tabular}

Double Star Catalog (WDS; Mason et al. 2001) and later by McCarthy \& Zuckerman (2004) in their coronagraphic search for brown dwarf companions, although no astrometry is provided in the latter. The WDS catalog lists a companion to the M2.4 primary with a contrast of 2.9 mag in the optical at a separation of $2^{\prime \prime} .5-2^{\prime \prime} .9$ and a position angle of $290^{\circ}-291^{\circ}$ from two epochs in 1995 and 1998. We confirm the physical separation of the pair and detect modest orbital motion. Unresolved light curves of GJ $3030 \mathrm{AB}$ from the HATNet survey (Hartman et al. 2011) reveal a photometric period of $6.166 \pm$ 
0.014 days likely corresponding to the rotation period of the primary.

NLTT 1875 (2MASS J00350487+5953079). Shkolnik et al. (2012) proposed this M4.3e star as a candidate kinematic member of IC 2391 based on their measured radial velocity $\left(-1.0 \pm 0.1 \mathrm{~km} \mathrm{~s}^{-1}\right)$ and its photometric distance of $26 \pm$ 6 pc. Recently, Dittmann et al. (2013) presented a trigonometric parallax of $38.3 \pm 2.2 \mathrm{pc}$ to NLTT 1875 . At this revised distance, the star's $U, V$, and $W$ space velocities are $\{-34 \pm 2,-21.8 \pm$ $\left.1.2,-3.1 \pm 0.3 \mathrm{~km} \mathrm{~s}^{-1}\right\}$, respectively, which do not correspond to any known YMGs. Moreover, it does not appear overluminous compared to normal main-sequence stars on the $M_{V}$ vs. $V-K_{S}$ diagram. We therefore adopt a wider age range of 100-500 Myr for this star.

G 271-110 (2MASS J01365529-0647363). This active M4 star is a very wide (14,600 AU) companion to EX Cet (AlonsoFloriano et al. 2011), a young G5 star with an Hipparcos distance of $24.0 \pm 0.4 \mathrm{pc}$. One faint $(\Delta K=13.8 \pm 1.0 \mathrm{mag})$ point source was identified at a separation of 6.'662 \pm 0.003 (160 AU) and a P.A. of $23.18 \pm 0.20$ from this star in our first epoch of imaging, but we were unable to recover it in several follow-up attempts because conditions were worse or integration times were insufficient.

1RXS J022735.8+471021 (2MASS J02273726+4710045). Based on the radial velocity of $-6.0 \pm 0.7$ from Shkolnik et al. (2012) and the parallactic distance of $27.4 \pm 1.7 \mathrm{~km}$ $\mathrm{s}^{-1}$ from Dittmann et al. (2013), the $U V W$ space velocities for the M4.6 star 1RXS J022735.8+471021 are $\{-7.9 \pm 1.2$, $\left.-23.9 \pm 1.6,-14.7 \pm 1.4 \mathrm{~km} \mathrm{~s}^{-1}\right\}$. These agree well with the AB Dor moving group (e.g., Torres et al. 2008), so we assign 1RXS J022735.8+471021 as a probable member of this moving group.

2MASS J03033668-2535329 AB. Makarov \& Kaplan (2005) first noted this M0 star as a likely binary from significant differences between Hipparcos and Tycho proper motions. This 0.83 binary $\left(\Delta K_{S}=2.99 \pm 0.06 \mathrm{mag}\right)$ was later resolved as part of the Astralux Lucky imaging survey by Bergfors et al. (2010) and Janson et al. (2012). Their astrometry from $2008.88(\rho=0.234 \pm 0.005, \theta=7.6 \pm 0.3)$ and $2010.08(\rho=$ $0.834 \pm 0$. '.005, $\theta=3.5 \pm 0.3$ ) together with our measurements from 2011 reveal a constant separation but a P.A. changing by $\approx 3^{\circ} \mathrm{yr}^{-1}$.

2MASS J04220833-2849053 AB. This star is a 0.74 equalflux K7Ve binary system. Torres et al. (2006) found strong $\mathrm{H} \alpha$ emission $(\mathrm{EW}=12 \AA)$ and $\mathrm{Li} \lambda 6708$ absorption $(E W=70 \mathrm{~m} \AA)$. Based on the stars' $V-I$ color of 1.2 mag from UCAC4, the Li depletion implies an age consistent with the Pleiades (Torres et al. 2008). We therefore adopt a conservative age range of 50-200 Myr for this system.

2MASS J04472312-2750358 and 2MASS J044722662750295. 2MASS J04472312-2750358 is the M2Ve secondary companion to the bright M0V star 2MASS J04472266-2750295 separated by 8 .'8. The stars share similar radial velocities and proper motions (Torres et al. 2006), and imaging dating to the early twentieth century shows some orbital motion (Mason et al. 2001). The system was detected by ROSAT and both components are detected in GALEX. The primary shows no $\mathrm{H} \alpha$ emission but Torres et al. (2006) found the companion is in emission, suggesting an upper age limit of $\sim 1.2$ Gyr. We therefore adopt the lower limit of 400 Myr from Shkolnik et al. (2009) and an upper limit of 1.2 Gyr for the system.

$L$ 449-1 AB (2MASS J05172292-3521545). This nearby (11.9 pc; Riedel et al. 2014) active pair of mid-M dwarfs was first noted by Scholz et al. (2005a). Riedel et al. (2014) identify a close stellar companion to the M4.0e primary at a separation of 47 mas from HST Fine Guidance Sensor interferometry from 2008. We did not resolve the companion in our HiCIAO observations from 2011. Interestingly, deep VLT/SINFONI observations from 1.4-2.5 $\mu \mathrm{m}$ by Janson et al. (2008) revealed a candidate marginally resolved ( $\approx 50$ mas) lowcontrast companion, though they attribute it to a PSF artifact. Riedel et al. (2014) find no evidence the system is particularly young and tentatively associate it with the UMa moving group based on its kinematics.

AP Col (2MASS J06045215-3433360). Scholz et al. (2005a) first drew attention to this active, optically variable M4.5 star because of its strong X-ray emission and proximity to the Sun. Riedel et al. (2011) measured a parallactic distance of $8.4 \mathrm{pc}$ and kinematically associate it with the young ( $40-50 \mathrm{Myr})$ Argus or IC 2391 moving groups. The origin and relationship of these two groups remains ambiguous (see Section 4.1 of Riedel et al. 2011 for a detailed discussion), but because of its proximity to Earth compared to typical IC 2391 members $(\sim 150 \mathrm{pc})$, we adopt Argus as the physical association. Deep AO imaging of AP Col by Quanz et al. (2012) did not reveal any planetary companions down to contrasts of $\Delta L^{\prime} \sim 11 \mathrm{mag}$ at $0^{\prime \prime} .5$, corresponding to planetary masses near $1 M_{\text {Jup }}$. Our HiCIAO observations in $H$ band reach a sensitivity of $13.5 \mathrm{mag}$ at $1^{\prime \prime}$ and we identify a single wide candidate companion at 7".1 (60 AU). Our follow-up astrometry at Keck shows it is a background star.

$1 R X S \quad J 091744.5+461229 \quad A B \quad(2 M A S S \quad J 09174473+$ 4612246). This M2.5 star was resolved into a 0.25 binary by Janson et al. (2012), who also confirmed the physical nature of the pair from two epochs of astrometry in 2008 and 2009. We detect modest orbital motion with our new astrometry. A rotational period of 0.562 days for the unresolved system was measured in the HATNet survey (Hartman et al. 2011).

GJ $354.1 B$ (2MASS J09324827+2659443). This star is a widely separated $\left(72^{\prime \prime}, \approx 1300 \mathrm{AU}\right)$ M5.5 companion to the young K0 star DX Leo (Gaidos 1998; Montes et al. 2001; Lowrance et al. 2005). The primary has a long history of potential kinematic matches to YMGs: Gaidos et al. (2000) list it as a candidate member of the Pleiades; Montes et al. (2001) and Maldonado et al. (2010) broadly associate it with the Local Association; Gaidos (1998) and Fuhrmann (2004) link it with the Her-Lyr group (though this is refuted by López-Santiago et al. 2006); Nakajima \& Morino (2012) find Tuc-Hor to be the best match; and Brandt et al. (2014) link it with Columba. While the $U V W$ kinematics of DX Leo are in good agreement with members of the Carina, Tuc-Hor, and Columba YMGs, its $X Y Z$ space positions do not entirely agree with a single group. Because of its close kinematic agreement with Carina members, we adopt that association and age ( $\approx 30 \mathrm{Myr})$ for GJ $354.1 \mathrm{~B}$, though a complete kinematic traceback analysis is needed to confirm this.

PYC J09362+3731 AB (2MASS J09361593+3731456; HIP 47133). This star is an equal-mass M0.5 SB2 system identified by Schlieder et al. (2012b) and Schlieder et al. (2012c) as a likely member of the $\beta$ Pic moving group based on its $U V W$ kinematics. However, Malo et al. (2013) note that the spatial position of PYC J09362+3731 AB disagrees with established members by $\sim 40 \mathrm{pc}$. This casts doubt on the membership of PYC J09362+3731 AB, especially since the activity detected by ROSAT and GALEX could be a result of tidal interactions rather than youth. We therefore assume it is a member of 
the field for this study and adopt a conservative age range of $10 \mathrm{Myr}-10 \mathrm{Gyr}$.

NLTT 22741 A (2MASS J09510459+3558098). LP 261-75 A is an active M4.5e star with an L6.5 companion separated by $12^{\prime \prime}$ (Reid \& Walkowicz 2006). At a distance of 33 pc (Bowler et al. 2013; Dittmann et al. 2013; F. Vrba, in preparation), this corresponds to $\approx 360 \mathrm{AU}$ in projected separation. Combining its distance and radial velocity from Shkolnik et al. (2012) gives $U V W$ space velocities of $\{-14.1 \pm 0.7,-24.3 \pm 1.5,-1.1 \pm$ $0.7\} \mathrm{km} \mathrm{s}^{-1}$. The $U$ and $V$ velocities are consistent with the Columba association, but differ by $\approx 5 \mathrm{~km} \mathrm{~s}^{-1}$ in $W$. Note, however, that the NIR spectrum of NLTT 22741 B (L4.5 \pm 1.0 spectral type) from Bowler et al. (2013) does not have the angular $H$-band features expected for a young $(\lesssim 100 \mathrm{Myr})$ brown dwarf. Lacking a likely YMG match, we therefore adopt the age estimate of 100-200 Myr from Reid \& Walkowicz (2006) for this system.

GJ $2079 A B$ (2MASS J10141918+2104297). This active M0.5 \pm 0.5 star (also known as DK Leo, HIP 50156) has a parallactic distance of $23.1 \pm 0.1 \mathrm{pc}$ (Perryman et al. 1997; van Leeuwen 2007). Makarov \& Kaplan (2005) and Frankowski et al. (2007) found evidence for a close astrometric companion based on differences between Hipparcos and Tycho-2 proper motions. Similarly, Shkolnik et al. (2012) identified GJ 2079 as an SB1 from variable radial velocity measurements spanning a decade. We resolved the likely culprit with $\mathrm{AO}$ imaging at Keck: a tight ( $~ 90$ mas) companion with a $K_{S}$-band contrast of $1.8 \mathrm{mag}$. The system is unresolved in our 2011 December 28 UT Subaru data, but two epochs at Keck (obtained before and after our HiCIAO data on 2011 March 25 UT and 2013 February 4 UT) separated by $\sim 2$ yr show substantial orbital motion. GJ 2079 was also imaged by the Subaru SEEDS program on 2011 December 24 UT - just a week before our own nondetection with HiCIAO reported here-and the companion was not resolved; Brandt et al. (2014) report an upper limit of $\sim 20$ mas, suggesting GJ 2079 B had moved too close to the primary to resolve at that epoch.

Schlieder et al. (2012b) identify GJ 2079 as a probable member of the $\beta$ Pic YMG, but Shkolnik et al. (2012) suggest the Carina YMG is more likely based on their more recent radial velocity measurement, the lack of $\mathrm{Li}$ absorption, and weak $\mathrm{H} \alpha$ emission (see note $k$ in their Table 6). Similarly, Malo et al. (2013) propose GJ 2079 is a member of the Columba group regardless of its (varying) radial velocities. Since GJ 2079 is a close binary, continued monitoring is clearly needed to derive a systemic radial velocity before reassessing its kinematic membership to YMGs. For this work we follow Shkolnik et al. in adopting GJ $2079 \mathrm{AB}$ as a member of the Carina YMG with an age of $\sim 30 \mathrm{Myr}$.

2MASS J11240434+3808108. This M4.5 star has a known M8.5 companion located at $8.3(\approx 170$ AU given its photometric distance of $\approx 20$ pc), 2MASS J11240487+3808054 (Close et al. 2003; Cruz et al. 2003). In addition to their common proper motion, the radial velocity of the companion $\left(-14 \pm 3 \mathrm{~km} \mathrm{~s}^{-1}\right)$ measured by Reiners \& Basri (2009) agrees with that of the primary $\left(-11.5 \pm 0.5 \mathrm{~km} \mathrm{~s}^{-1}\right.$ ) from Shkolnik et al. (2012). Shkolnik et al. (2009) assign an age range of 40-300 Myr for the primary from its high X-ray emission and lack of spectroscopic indicators of youth, while Shkolnik et al. (2012) tentatively assign it to the Ursa Major moving group $(\sim 500 \pm$ 100 Myr; King et al. 2003) from its kinematics. Burgasser et al. (2004) obtained a low-resolution near-infrared spectrum of the companion, which does not show obvious signs of low gravity in the form of an angular $H$-band shape, supporting an age $\gtrsim 100$ Myr (e.g., Allers \& Liu 2013). The HATNet survey measured a fast rotation period of 0.475 days for the primary (Hartman et al. 2011); unfortunately, rotation periods become unreliable age indicators for stars that are fully convective (Irwin et al. 2011).

The 2MASS $H$-band spectrophotometric distance to the companion 2MASS J11240487+3808054 B is $20.3 \pm 1.3 \mathrm{pc}$ using the relations from Dupuy \& Liu (2012). (This error incorporates the rms spread from Dupuy \& Liu, a spectral type uncertainty of 0.5 subclasses, and the photometric uncertainty.) At this distance, the $U V W$ space velocities of the system $\left(\{14.8 \pm 0.7,2.8 \pm 0.3,-6.7 \pm 0.5\} \mathrm{km} \mathrm{s}^{-1}\right)$ are an excellent kinematic match with Ursa Major (see Table 2 of Eiff \& Guenther 2009). We therefore adopt an age of $500 \pm 100 \mathrm{Myr}$ for this system. The corresponding luminosity of the companion is $\log L_{\mathrm{Bol}} / L_{\odot}=-3.35 \pm 0.06 \mathrm{dex}$, which translates into a mass of $81 \pm 5 M_{\text {Jup }}$ using evolutionary models from Burrows et al. (1997). This is very near the substellar boundary; however, the probability that the mass is below $75 M_{\text {Jup }}$ is only $15 \%$. Regardless, it was not detected in our high-contrast imaging due to its large angular separation so does not enter into our statistical analysis.

TWA 30 A (2MASS J11321831-3019518). This young M5 star was identified as a new member of the TWA moving group by Looper et al. (2010b). It exhibits Li I $\lambda 6708$ absorption and forbidden optical line emission, probably a result of outflow activity. Looper et al. (2010a) identified a very wide ( 3400 AU) companion, which shows similar forbidden lines emission. Although TWA $30 \mathrm{~B}$ is much fainter (5 mag in $K$ band), its earlier spectral type (M4) and variable reddening suggests it harbors an edge-on disk. Our NIRC 2 data show that TWA $30 \mathrm{~A}$ is single down to $\approx 0$ '.06 (2.5 AU), and deep imaging did not reveal any substellar candidates. Note that TWA $30 \mathrm{~A}$ is strongly variable in the NIR (Looper et al. 2010a). This affects the conversion of relative contrast curves to absolute contrasts and companion mass sensitivities. For this work we have adopted the 2MASS $K_{S}$-band magnitude for the primary, which may not accurately represent the apparent brightness of TWA 30 A during our deep ADI observation.

2MASS J12062214-1314559 AB. This M3.5 system was first resolved from a single epoch of imaging by Janson et al. (2012) into a $\approx 0$ ". 4 binary with a $z$-band contrast of 2.2 mag. We confirm the physical nature of the pair and detect orbital motion relative to the astrometry from Janson et al. at epoch $2010.11(\rho=$ $0.420 \pm 0$.'003, $\theta=65.9 \pm 0.3$ ). Riaz et al. (2006) identified 2MASS J12062214-1314559 AB as a chromospherically active $\operatorname{star}(\mathrm{EW}(\mathrm{H} \alpha)=-4.9 \AA)$.

LHS 2613 (2MASS J12424996+4153469). This single, Xray active M4.0 dwarf has been identified by E. L. Shkolnik et al. (in preparation) as a possible nearby young star. Its parallactic distance of $10.6 \pm 1.3 \mathrm{pc}$ (van Altena et al. 1995) combined with its measured radial velocity of $-4.0 \pm 0.2 \mathrm{~km}$ $\mathrm{s}^{-1}$ (E. L. Shkolnik et al., in preparation) imply $U V W$ kinematics of $\{-23 \pm 3,-13.7 \pm 1.6,-5.2 \pm 0.3\} \mathrm{km} \mathrm{s}^{-1}$, which agree well with the Argus YMG. The large uncertainty in $U$ is mostly influenced by the error in the distance to the system. Association with the $\sim 40$ Myr Argus group agrees with the star's placement on the color-magnitude diagram; with $V=12.4$ mag (Zacharias et al. 2013) and $M_{V}=12.3 \mathrm{mag}$, LHS 2613 lies $\sim 0.5-1 \mathrm{mag}$ above the main sequence given its $V-K_{S}$ color of 5.16 (see, e.g., Figure 4 from Riedel et al. 2014). This is further bolstered by an $86 \%$ membership probability by the BANYAN II web tool from 
Gagné et al. (2014). ${ }^{15}$ If confirmed with a more precise distance, LHS 2613 will be among the nearest pre-main-sequence stars.

GJ 1167 A (2MASS J13093495+2859065). A 194" companion to the M3.5 star GJ 1167 A at a P.A. of $23^{\circ}$ (LP 322-835; GJ 1167 B) is listed in the Washington Double Star catalog (Mason et al. 2001). However, the proper motion of GJ 1167

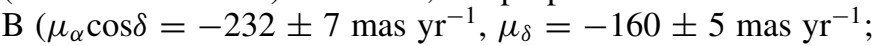
Monet et al. 2003) disagrees with GJ $1167 \mathrm{~A}\left(\mu_{\alpha} \cos \delta=-338 \pm\right.$

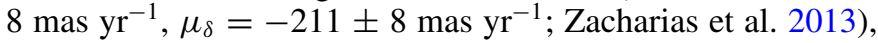
so the pair are unlikely to be physically related.

$G$ 227-22 (2MASS J18021660+6415445). The parallactic distance of $8.5 \pm 0.3$ pc to G 227-22 from Dittmann et al. (2013) combined with the radial velocity measurement of $-1.2 \pm$ $0.2 \mathrm{~km} \mathrm{~s}^{-1}$ from Shkolnik et al. (2012) yield $U V W$ space velocities of $\{15.0 \pm 0.6,4.2 \pm 0.3,-8.3 \pm 0.4\} \mathrm{km} \mathrm{s}^{-1}$. These are in excellent agreement with the Ursa Major moving group (Eiff \& Guenther 2009), so we assign G 227-22 as a likely member of that association and adopt the group age of $500 \pm$ 100 Myr for this star.

2MASS J20003177+5921289 AB. This near equal-flux M4.1type 0'.3 binary was first identified by Janson et al. (2012) from imaging in 2008 and 2009. Our 2010 data show continued outward orbital motion by $\approx 20$ mas in separation and $\approx 5^{\circ}$ in P.A.

NLTT 48651 (2MASS J20043077-2342018). NLTT 48651 is a single M4.5 dwarf detected by ROSAT and GALEX and identified by E. L. Shkolnik et al. (in preparation) as a possible nearby young star. The radial velocity of $-7.5 \pm 0.7 \mathrm{~km} \mathrm{~s}^{-1}$ measured by Shkolnik et al. enable partial constraints on the star's kinematics, which agree well with AB Dor moving group members at a distance of $\sim 18 \mathrm{pc}$. Indeed, the BANYAN II web tool suggests an AB Dor membership probability of 93\%, so we adopt the AB Dor age of $120 \pm 10 \mathrm{Myr}$ for this star. A parallax will be needed for unambiguous confirmation of group membership.

2MASS J20284361-1128307. This X-ray active M3.5 dwarf has mostly gone unnoticed in the literature. Riaz et al. (2006) measured moderately strong $\mathrm{H} \alpha$ emission $(6.3 \AA)$ and, more recently, Riedel et al. (2014) presented a trigonometric distance of $18.8 \pm 0.6 \mathrm{pc}$. Although lacking a radial velocity, Riedel et al. argue that this star is a probable member of the Argus association based on its position on the HR diagram and partially constrained kinematics. Assuming membership to Argus, they predict a radial velocity of $-25.4 \mathrm{~km} \mathrm{~s}^{-1}$. E. L. Shkolnik et al. (in preparation) measure a radial velocity of $-25.2 \pm 0.3 \mathrm{~km}$ $\mathrm{s}^{-1}$ implying $U V W$ kinematics of $\{-24.4 \pm 0.4,-17.7 \pm 0.5$, $-3.8 \pm 0.7\} \mathrm{km} \mathrm{s}^{-1}$. These are in good agreement with the Argus moving group, so we consider 2MASS J20284361-1128307 a likely member.

NLTT 50066 AB (2MASS J20531465-0221218). This M3.0 equal flux binary $(\Delta H=0.1 \mathrm{mag})$ was first resolved by Janson et al. (2012). The pair has undergone significant orbital evolution since the Janson et al. first epoch in 2008. Its parallactic distance of $37.9 \pm 5.7 \mathrm{pc}$ (Shkolnik et al. 2012) implies a physical separation of $~ 3-5$ AU. With an expected orbital period of $\sim 10-20 \mathrm{yr}$, astrometric monitoring should continue in order to yield a dynamical mass.

G 68-46 (2MASS J23512227+2344207). Lacking a parallax for $\mathrm{G}$ 68-46, previous studies have tentatively associated this active M4.0e star with the $\beta$ Pic (Malo et al. 2013) and ChaNear (Shkolnik et al. 2012) moving groups. However, based

\footnotetext{
15 http://www.astro.umontreal.ca/ gagne/banyanII.php
}

on the trigonometric distance of $21.0 \pm 1.3 \mathrm{pc}$ from Dittmann et al. (2013) and radial velocity of $-2.1 \pm 0.5 \mathrm{~km} \mathrm{~s}^{-1}$ from Shkolnik et al. (2012), we find that the $U V W$ kinematics of $\mathrm{G}$ $68-46\left(\{-19.4 \pm 1.5,-16.4 \pm 1.1,-10.5 \pm 1.0\} \mathrm{km} \mathrm{s}^{-1}\right) \mathrm{do}$ not match those of any nearby YMGs. We therefore adopt the age estimate of 35-300 Myr from Shkolnik et al. (2009).

\section{REFERENCES}

Abazajian, K. N., Adelman-McCarthy, J. K., Agüeros, M. A., et al. 2009, ApJSS, 182,543

Abt, H. A. 2006, ApJ, 651, 1151

Alibert, Y., Mordasini, C., Benz, W., \& Winisdoerffer, C. 2005, A\&A, 434, 343 Allard, F., Homeier, D., \& Freytag, B. 2011, in ASP Conf Ser. 448, 16th Cambridge Workshop on Cool Stars, Stellar Systems, and the Sun, ed. C. Johns-Krull, M. K. Browning, \& A. A. West (San Francisco, CA: ASP), 91 Allen, P. R., \& Reid, I. N. 2008, AJ, 135, 2024

Allers, K. N., Jaffe, D. T., Luhman, K. L., et al. 2007, ApJ, 657, 511

Allers, K. N., \& Liu, M. C. 2013, ApJ, 772, 79

Alonso-Floriano, F. J., Caballero, J. A., \& Montes, D. 2011, in Stellar Clusters \& Associations: A RIA Workshop on Gaia, ed. E. J. Alfaro Navarro, A. T. Gallego Calvente, \& M. R. Zapatero Osorio, 344

Anderson, J., \& King, I. R. 2003, PASP, 115, 113

Andrews, S. M., Rosenfeld, K. A., Kraus, A. L., \& Wilner, D. J. 2013, ApJ, 771,129

Baraffe, I., Chabrier, G., Allard, F., \& Hauschildt, P. H. 1998, A\&A, 337, 403

Baraffe, I., Chabrier, G., Barman, T. S., Allard, F., \& Hauschildt, P. H. 2003, A\&A, 402, 701

Barenfeld, S. A., Bubar, E. J., Mamajek, E. E., \& Young, P. A. 2013, ApJ, 766,6

Barrado y Navascués, D. 1998, A\&A, 339, 831

Bate, M. R. 2009, MNRAS, 392, 590

Bate, M. R. 2012, MNRAS, 419, 3115

Bergfors, C., Brandner, W., Janson, M., et al. 2010, A\&A, 520, A54

Berta, Z. K., Irwin, J., \& Charbonneau, D. 2013, ApJ, 775, 91

Biller, B., Artigau, E., Wahhaj, Z., et al. 2008, Proc. SPIE, 7015, 70156Q

Biller, B. A., Close, L. M., Masciadri, E., et al. 2007, ApJSS, 173, 143

Biller, B. A., Liu, M. C., Wahhaj, Z., et al. 2013, ApJ, 777, 160

Binks, A. S., \& Jeffries, R. D. 2014, MNRAS, 438, L11

Bochanski, J. J., Hawley, S. L., Covey, K. R., et al. 2010, AJ, 139, 2679

Bodenheimer, P., D'angelo, G., Lissauer, J. J., Fortney, J. J., \& Saumon, D. 2013, ApJ, 770, 120

Boley, A. C. 2009, ApJL, 695, L53

Bonfils, X., Delfosse, X., Udry, S., et al. 2013, A\&A, 549, 109

Boss, A. P. 1997, Sci, 276, 1836

Boss, A. P. 2007, ApJL, 661, L73

Boss, A. P. 2011, ApJ, 731, 74

Bowler, B. P., Johnson, J. A., Marcy, G. W., et al. 2010, ApJ, 709, 396 Bowler, B. P., Liu, M. C., Shkolnik, E. L., \& Dupuy, T. J. 2013, ApJ, 774, 55 Bowler, B. P., Liu, M. C., Shkolnik, E. L., \& Tamura, M. 2012a, ApJ, 756, 69 Bowler, B. P., Liu, M. C., Shkolnik, E. L., et al. 2012b, ApJ, 753, 142 Brandt, T. D., Kuzuhara, M., McElwain, M. W., et al. 2014, ApJ, 786, 1 Brandt, T. D., McElwain, M. W., Turner, E. L., et al. 2013, ApJ, 764, 183 Burgasser, A. J., McElwain, M. W., Kirkpatrick, J. D., et al. 2004, AJ, 127, 2856 Burrows, A., Marley, M., Hubbard, W. B., et al. 1997, ApJ, 491, 856 Buscombe, W. 1998, yCat (Evanston, IL: Northwestern Univ.) Butler, R. P., Johnson, J. A., Marcy, G. W., et al. 2006, PASP, 118, 1685 Butler, R. P., Vogt, S. S., Marcy, G. W., et al. 2004, ApJ, 617, 580 Cameron, P. B., Britton, M. C., \& Kulkarni, S. R. 2009, AJ, 137, 83 Cantrell, J. R., Henry, T. J., \& White, R. J. 2013, AJ, 146, 99 Cassan, A., Kubas, D., Beaulieu, J.-P., et al. 2012, Natur, 481, 167 Chabrier, G., Baraffe, I., Allard, F., \& Hauschildt, P. 2000, ApJ, 542, 464 Charbonneau, D., Berta, Z. K., Irwin, J., et al. 2009, Natur, 462, 891 Chauvin, G., Lagrange, A.-M., Bonavita, M., et al. 2010, A\&A, 509, A52 Chauvin, G., Lagrange, A.-M., Dumas, C., et al. 2004, A\&A, 425, L29 Chauvin, G., Vigan, A., Bonnefoy, M., et al. 2014, arXiv:1405.1560 Clanton, C., \& Gaudi, B. S. 2014, ApJ, 791, 91

Close, L. M., Siegler, N., Freed, M., \& Biller, B. 2003, ApJ, 587, 407 Conroy, C., \& van Dokkum, P. G. 2012, ApJ, 760, 71

Crepp, J. R., \& Johnson, J. A. 2011, ApJ, 733, 126

Crepp, J. R., Johnson, J. A., Fischer, D. A., et al. 2012, ApJ, 751, 97

Crepp, J. R., Pueyo, L., Brenner, D., et al. 2011, ApJ, 729, 132

Cruz, K. L., Reid, I. N., Liebert, J., Kirkpatrick, J. D., \& Lowrance, P. J. 2003, AJ, 126,2421

Cumming, A., Butler, R. P., Marcy, G. W., et al. 2008, PASP, 120, 531 
Cushing, M. C., Rayner, J. T., \& Vacca, W. D. 2005, ApJ, 623, 1115 Cushing, M. C., Vacca, W. D., \& Rayner, J. T. 2004, PASP, 116, 362 Daemgen, S., Siegler, N., Reid, I. N., \& Close, L. M. 2007, ApJ, 654, 558 Degennaro, S., Hippel, T. V., Jefferys, W. H., et al. 2009, ApJ, 696, 12 Delfosse, X., Beuzit, J.-L., Marchal, L., et al. 2004, in ASP Conf. Ser. 318, Spectroscopically and Spatially Resolving the Components of Close Binary Stars, ed. R. W. Hilditch, H. Hensberge, \& K. Pavlovski (San Francisco, CA: ASP), 166

Delorme, P., Gagné, J., Girard, J. H., et al. 2013, A\&A, 553, L5

Delorme, P., Lagrange, A. M., Chauvin, G., et al. 2012, A\&A, 539, A72

Dieterich, S. B., Henry, T. J., Golimowski, D. A., Krist, J. E., \& Tanner, A. M. 2012, AJ, 144, 64

Diolaiti, E., Bendinelli, O., Bonaccini, D., et al. 2000, A\&AS, 147, 335

Dittmann, J. A., Irwin, J. M., Charbonneau, D., \& Berta-Thompson, Z. K. 2014, ApJ, 784, 156

Dodson-Robinson, S. E., Veras, D., Ford, E. B., \& Beichman, C. A. 2009, ApJ, 707, 79

Dressing, C. D., \& Charbonneau, D. 2013, ApJ, 767, 95

Duchêne, G. 2010, ApJL, 709, L114

Duchêne, G., \& Kraus, A. 2013, ARA\&A, 51, 269

Dupuy, T. J., \& Kraus, A. L. 2013, Sci, 341, 1492

Dupuy, T. J., \& Liu, M. C. 2011, ApJ, 733, 122

Dupuy, T. J., \& Liu, M. C. 2012, ApJSS, 201, 19

Dupuy, T. J., Liu, M. C., \& Ireland, M. J. 2009, ApJ, 692, 729

Dupuy, T. J., Liu, M. C., \& Ireland, M. J. 2014, ApJ, 790, 133

Durisen, R. H., Boss, A. P., Mayer, L., et al. 2007, in Protostars and Planets V, ed. e. B. Reipurth, D. Jewitt, \& K. Keil (Tucson, AZ: Univ. Arizona Press), 607

Eggen, O. J. 1958, MNRAS, 118, 65

Eggen, O. J. 1983, AJ, 88, 642

Eggen, O. J. 1998, AJ, 116, 284

Egner, S., Ikeda, Y., Watanabe, M., et al. 2010, Proc. SPIE, 7736, 77364V

Ehrenreich, D., Lagrange, A.-M., Montagnier, G., et al. 2010, A\&A, 523, A73

Eiff, M. A.-V., \& Guenther, E. W. 2009, A\&A, 508, 677

Endl, M., Cochran, W. D., Kürster, M., et al. 2006, ApJ, 649, 436

Famaey, B., Jorissen, A., Luri, X., et al. 2005, A\&A, 430, 165

Fischer, D. A., \& Marcy, G. W. 1992, ApJ, 396, 178

Fortney, J. J., Marley, M. S., Saumon, D., \& Lodders, K. 2008, ApJ, 683, 1104 Frankowski, A., Jancart, S., \& Jorissen, A. 2007, A\&A, 464, 377

Fu, H., Cooray, A., Feruglio, C., et al. 2014, Natur, 498, 338

Fuhrmann, K. 2004, AN, 325, 3

Gagné, J., LaFreniere, D., Doyon, R., Malo, L., \& Artigau, E. 2014, ApJ, 783,121

Gaidos, E., Fischer, D. A., Mann, A. W., \& Howard, A. W. 2013, ApJ, 771, 18

Gaidos, E. J. 1998, PASP, 110, 1259

Gaidos, E. J., Henry, G. W., \& Henry, S. M. 2000, AJ, 120, 1006

Giclas, H. L., Burnham, R., \& Thomas, N. G. 1963, LowOB, 6, 1

Giclas, H. L., Burnham, R., \& Thomas, N. G. 1964, LowOB, 6, 135

Giclas, H. L., Burnham, R., \& Thomas, N. G. 1966a, LowOB, 6, 271

Giclas, H. L., Burnham, R., \& Thomas, N. G. 1966b, LowOB, 6, 233

Golimowski, D. A., Leggett, S. K., Marley, M. S., et al. 2004, AJ, 127, 3516

Gould, A., Dong, S., Gaudi, B. S., et al. 2010, ApJ, 720, 1073

Gould, A., \& Loeb, A. 1992, ApJ, 396, 104

Han, C. 2006, ApJ, 644, 1232

Han, C. 2009, ApJ, 700, 945

Han, C., Jung, Y. K., Udalski, A., et al. 2013, ApJ, 778, 38

Hartman, J. D., Bakos, G. Á., Noyes, R. W., et al. 2011, AJ, 141, 166

Hawley, S. L., Gizis, J. E., \& Reid, I. N. 1996, AJ, 112, 2799

Hayano, Y., Takami, H., Oya, S., et al. 2010, Proc. SPIE, 7736, 77360N

Henry, T. J., Jao, W.-C., Subasavage, J. P., et al. 2006, AJ, 132, 2360

Hinkley, S., Oppenheimer, B. R., Soummer, R., et al. 2007, ApJ, 654, 633

Hodapp, K. W., Suzuki, R., Tamura, M., et al. 2008, Proc. SPIE, 7014, 701419

Holman, M. J., \& Wiegert, P. A. 1999, AJ, 117, 621

Howard, A. W., Marcy, G. W., Johnson, J. A., et al. 2010, Sci, 330, 653

Ida, S., \& Lin, D. N. C. 2005, ApJ, 626, 1045

Irwin, J., Berta, Z. K., Burke, C. J., et al. 2011, ApJ, 727, 56

Janson, M., Bonavita, M., Klahr, H., et al. 2011, ApJ, 736, 89

Janson, M., Brandner, W., \& Henning, T. 2008, A\&A, 478, 597

Janson, M., Hormuth, F., Bergfors, C., et al. 2012, ApJ, 754, 44

Johnson, J. A., Aller, K. M., Howard, A. W., \& Crepp, J. R. 2010, PASP, 122,905

Johnson, J. A., Butler, R. P., Marcy, G. W., et al. 2007, ApJ, 670, 833

Joshi, M. M., Haberle, R. M., \& Reynolds, R. T. 1997, Icar, 129, 450

Kalas, P., Graham, J. R., Chiang, E., et al. 2008, Sci, 322, 1345

Kennedy, G. M., \& Kenyon, S. J. 2008, ApJ, 673, 502

King, J. R., Villarreal, A. R., Soderblom, D. R., Gulliver, A. F., \& Adelman, S. J. 2003, AJ, 125, 1980
Kipping, D. M. 2013, MNRAS, 434, L51

Kirkpatrick, J. D., Cruz, K. L., Barman, T. S., et al. 2008, ApJ, 689, 1295

Kirkpatrick, J. D., Gelino, C. R., Cushing, M. C., et al. 2012, ApJ, 753, 156

Kiss, L. L., Moór, A., Szalai, T., et al. 2010, MNRAS, 411, 117

Kratter, K. M., Murray-Clay, R. A., \& Youdin, A. N. 2010, ApJ, 710, 1375

Kraus, A. L., Ireland, M. J., Hillenbrand, L. A., \& Martinache, F. 2012, ApJ, 745,19

Kraus, A. L., Shkolnik, E. L., Allers, K. N., \& Liu, M. C. 2014, AJ, 147, 146

Kraus, A. L., Tucker, R. A., Thompson, M. I., Craine, E. R., \& Hillenbrand, L. A. 2011, ApJ, 728, 48

Kuzuhara, M., Tamura, M., Kudo, T., et al. 2013, ApJ, 774, 11

Lada, C. J. 2006, ApJL, 640, L63

Lafrenière, D., Doyon, R., Marois, C., et al. 2007a, ApJ, 670, 1367

Lafrenière, D., Marois, C., Doyon, R., Nadeau, D., \& Artigau, É. 2007b, ApJ, 660,770

Lagrange, A.-M., Bonnefoy, M., Chauvin, G., et al. 2010, Sci, 329, 57

Larkin, J., Barczys, M., Krabbe, A., et al. 2006, Proc. SPIE, 6269, 42

Laughlin, G., Bodenheimer, P., \& Adams, F. C. 2004, ApJL, 612, L73

Law, N. M., Hodgkin, S. T., \& Mackay, C. D. 2008, MNRAS, 384, 150

Lebreton, Y., Fernandes, J., \& Lejeune, T. 2001, A\&A, 374, 540

Leggett, S. K., Currie, M. J., Varricatt, W. P., et al. 2006, MNRAS, 373, 781

Lépine, S., \& Gaidos, E. 2011, AJ, 142, 138

Lépine, S., Hilton, E. J., Mann, A. W., et al. 2013, AJ, 145, 102

Lépine, S., \& Simon, M. 2009, AJ, 137, 3632

Liu, M. C. 2004, Sci, 305, 1442

Liu, M. C. 2010a, Proc. SPIE, 7736, 77361K

Liu, M. C., Dupuy, T. J., Bowler, B. P., Leggett, S. K., \& Best, W. M. J. 2012, ApJ, 758,57

Liu, M. C., Dupuy, T. J., \& Ireland, M. J. 2008, ApJ, 689, 436

Liu, M. C., Dupuy, T. J., \& Leggett, S. K. 2010b, ApJ, 722, 311

Looper, D. L., Bochanski, J. J., Burgasser, A. J., et al. 2010a, AJ, 140, 1486

Looper, D. L., Burgasser, A. J., Kirkpatrick, J. D., \& Swift, B. J. 2007, ApJL, 669, L97

Looper, D. L., Mohanty, S., Bochanski, J. J., et al. 2010b, ApJ, 714, 45

López-Santiago, J., Montes, D., Crespo-Chacón, I., \& Fernández-Figueroa, M. J. 2006, ApJ, 643, 1160

Lovis, C., \& Mayor, M. 2007, A\&A, 472, 657

Lowrance, P. J., Becklin, E. E., Schneider, G., et al. 2005, AJ, 130, 1845

Luhman, K. L., Stauffer, J. R., \& Mamajek, E. E. 2005, ApJL, 628, L69

Macintosh, B., Graham, J. R., Ingraham, P., et al. 2014, PNAS, 111, 12661

Macintosh, B., Poyneer, L., Sivaramakrishnan, A., \& Marois, C. 2005, Proc. SPIE, 5903, 170

Makarov, V. V., \& Kaplan, G. H. 2005, AJ, 129, 2420

Maldonado, J., Martínez-Arnáiz, R. M., Eiroa, C., Montes, D., \& Montesinos, B. 2010, A\&A, 521, A12

Malo, L., Artigau, E., Doyon, R., et al. 2014a, ApJ, 788, 81

Malo, L., Doyon, R., Feiden, G. A., et al. 2014b, ApJ, 792, 37

Malo, L., Doyon, R., Lafrenière, D., et al. 2013, ApJ, 762, 88

Mamajek, E. E. 2005, ApJ, 634, 1385

Mamajek, E. E. 2012, ApJL, 754, L20

Mamajek, E. E., \& Bell, C. P. M. 2014, MNRAS, 445, 2169

Marley, M. S., Fortney, J. J., Hubickyj, O., Bodenheimer, P., \& Lissauer, J. J. 2007, ApJ, 655, 541

Marois, C., Doyon, R., Nadeau, D., et al. 2005, PASP, 117, 745

Marois, C., Doyon, R., Racine, R., \& Nadeau, D. 2000, PASP, 112, 91

Marois, C., Lafrenière, D., Doyon, R., Macintosh, B., \& Nadeau, D. 2006, ApJ, 641, 556

Marois, C., Macintosh, B., Barman, T., et al. 2008, Sci, 322, 1348

Marois, C., Macintosh, B., \& Véran, J.-P. 2010a, Proc. SPIE, 7736, 77361J

Marois, C., Zuckerman, B., Konopacky, Q. M., Macintosh, B., \& Barman, T. 2010b, Natur, 468, 1080

Masciadri, E., Mundt, R., Henning, T., Alvarez, C., \& Navascués, D. B. Y. 2005, ApJ, 625, 1004

Mason, B. D., Wycoff, G. L., Hartkopf, W. I., Douglass, G. G., \& Worley, C. E. 2001, AJ, 122, 3466

Mayer, L. 2002, Sci, 298, 1756

Mayor, M., Bonfils, X., Forveille, T., et al. 2009, A\&A, 507, 487

McCarthy, C., \& Zuckerman, B. 2004, AJ, 127, 2871

McCarthy, C., Zuckerman, B., \& Becklin, E. E. 2001, AJ, 121, 3259

McLean, I. S., Wilcox, M. K., Becklin, E. E., et al. 2000, ApJL, 533, L45

Metchev, S. A., \& Hillenbrand, L. A. 2009, ApJSS, 181, 62

Mieda, E., Wright, S. A., Larkin, J. E., et al. 2014, PASP, 126, 250

Mohanty, S., Ercolano, B., \& Turner, N. J. 2013, ApJ, 764, 65

Mollière, P., \& Mordasini, C. 2012, A\&A, 547, A105

Monet, D. G., Levine, S. E., Canzian, B., et al. 2003, AJ, 125, 984

Montes, D., López-Santiago, J., Fernández-Figueroa, M. J., \& Gálvez, M. C. 2001, A\&A, 379, 976 
Montet, B. T., Crepp, J. R., Johnson, J. A., Howard, A. W., \& Marcy, G. W. 2014, ApJ, 781, 28

Morton, T. D., \& Swift, J. 2014, ApJ, 791, 10

Muirhead, P. S., Johnson, J. A., Apps, K., et al. 2012, ApJ, 747, 144

Nakajima, T., \& Morino, J.-I. 2012, AJ, 143, 2

Naud, M.-E., Artigau, É., Malo, L., et al. 2014, ApJ, 787, 5

Navascués, D. B. Y. 2006, A\&A, 459, 511

Nero, D., \& Bjorkman, J. E. 2009, ApJL, 702, L163

Nielsen, E. L., \& Close, L. M. 2010, ApJ, 717, 878

Nielsen, E. L., Close, L. M., Biller, B. A., Masciadri, E., \& Lenzen, R. 2008, ApJ, 674, 466

Nielsen, E. L., Liu, M. C., Wahhaj, Z., et al. 2013, ApJ, 776, 4

Öpik, E. 1924, in Publications de L'Observatoire Astronomique de l'Universite de Tartu, Vol. 25, No. 6

Oppenheimer, B. R., \& Hinkley, S. 2009, ARA\&A, 47, 253

Ortega, V. G., Jilinski, E., Reza, R. D. L., \& Bazzanella, B. 2007, MNRAS, 377,441

Perryman, M. A. C., Brown, A. G. A., Lebreton, Y., et al. 1998, A\&A, 331, 81

Perryman, M. A. C., Lindegren, L., Kovalevsky, J., et al. 1997, A\&A, 323, L49

Pollack, J. B., Hubickyj, O., Bodenheimer, P., et al. 1996, Icar, 124, 62

Poveda, A., Herrera, M. A., Allen, C., Cordero, G., \& Lavalley, C. 1994, RMxAA, 28, 43

Pravdo, S. H., Shaklan, S. B., Wiktorowicz, S. J., et al. 2006, ApJ, 649, 389

Pueyo, L., Crepp, J. R., Vasisht, G., et al. 2012, ApJSS, 199, 6

Quanz, S. P., Crepp, J. R., Janson, M., et al. 2012, ApJ, 754, 127

Quintana, E. V., Barclay, T., Raymond, S. N., et al. 2014, Sci, 344, 277

Racine, R., Walker, G. A. H., Nadeau, D., Doyon, R., \& Marois, C. 1999, PASP, 111,587

Rameau, J., Chauvin, G., Lagrange, A.-M., et al. 2013a, A\&A, 553, A60

Rameau, J., Chauvin, G., Lagrange, A.-M., et al. 2013b, ApJL, 779, L26

Rayner, J. T., Cushing, M. C., \& Vacca, W. D. 2009, ApJSS, 185, 289

Rayner, J. T., Toomey, D. W., Onaka, P. M., et al. 2003, PASP, 115, 362

Rebolo, R., Osorio, M. R. Z., Madruga, S., et al. 1998, Sci, 282, 1309

Reid, I. N., Cruz, K. L., \& Allen, P. R. 2007, AJ, 133, 2825

Reid, I. N., Hawley, S. L., \& Gizis, J. E. 1995, AJ, 110, 1838

Reid, I. N., \& Walkowicz, L. M. 2006, PASP, 118, 671

Reiners, A., \& Basri, G. 2009, ApJ, 705, 1416

Riaz, B., Gizis, J. E., \& Harvin, J. 2006, AJ, 132, 866

Ribas, I. 2003, A\&A, 400, 297

Riedel, A. R., Finch, C. T., Henry, T. J., et al. 2014, AJ, 147, 85

Riedel, A. R., Murphy, S. J., Henry, T. J., et al. 2011, AJ, 142, 104

Riedel, A. R., Subasavage, J. P., Finch, C. T., et al. 2010, AJ, 140, 897

Rodriguez, D. R., Zuckerman, B., Kastner, J. H., et al. 2013, ApJ, 774, 101

Roeser, S., Demleitner, M., \& Schilbach, E. 2010, AJ, 139, 2440

Röser, S., Schilbach, E., Piskunov, A. E., Kharchenko, N. V., \& Scholz, R.-D. 2011, A\&A, 531, A92

Röser, S., Schilbach, E., Schwan, H., et al. 2008, A\&A, 488, 401

Saumon, D., \& Marley, M. S. 2008, ApJ, 689, 1327

Schlieder, J. E., Lépine, S., Rice, E., et al. 2012a, AJ, 143, 114

Schlieder, J. E., Lépine, S., \& Simon, M. 2010, AJ, 140, 119

Schlieder, J. E., Lépine, S., \& Simon, M. 2012b, AJ, 143, 80

Schlieder, J. E., Lépine, S., \& Simon, M. 2012c, AJ, 144, 109

Scholz, R.-D., Curto, G. L., Méndez, R. A., et al. 2005a, A\&A, 439, 1127

Scholz, R.-D., Meusinger, H., \& Jahreiß, H. 2005b, A\&A, 442, 211

Shkolnik, E., Liu, M. C., \& Reid, I. N. 2009, ApJ, 699, 649

Shkolnik, E. L., Anglada-Escudé, G., Liu, M. C., et al. 2012, ApJ, 758, 56

Shkolnik, E. L., Hebb, L., Liu, M. C., Reid, I. N., \& Cameron, A. C. 2010, ApJ, 716,1522
Shkolnik, E. L., Liu, M. C., Reid, I. N., Dupuy, T., \& Weinberger, A. J. 2011, ApJ 727, 6

Silva, G. M. D., D’Orazi, V., Melo, C., et al. 2013, MNRAS, 431, 1005

Simons, D. A., \& Tokunaga, A. 2002, PASP, 114, 169

Skemer, A. J., Hinz, P., Esposito, S., et al. 2014, Proc. SPIE, 9148, 91480L

Skrutskie, M. F., Cutri, R. M., Stiening, R., et al. 2006, AJ, 131, 1163

Slesnick, C. L., Hillenbrand, L. A., \& Carpenter, J. M. 2004, ApJ, 610,1045

Soderblom, D. R., \& Mayor, M. 1993, AJ, 105, 226

Soummer, R., Hagan, J. B., Pueyo, L., et al. 2011, ApJ, 741, 55

Sparks, W. B., \& Ford, H. C. 2002, ApJ, 578, 543

Spiegel, D. S., \& Burrows, A. 2012, ApJ, 745, 174

Stamatellos, D., \& Whitworth, A. P. 2009, MNRAS, 392, 413

Stephenson, C. B. 1986, AJ, 92, 139

Stetson, P. B. 1987, PASP, 99, 191

Sumi, T., Kamiya, K., Bennett, D. P., et al. 2011, Natur, 473, 349

Suzuki, R., Kudo, T., Hashimoto, J., et al. 2010, Proc. SPIE, 7735, 773530

Swift, J. J., Johnson, J. A., Morton, T. D., et al. 2013, ApJ, 764, 105

Tamura, M., Hodapp, K., Takami, H., et al. 2006, Proc. SPIE, 6269, 62690V

Thatte, N., Abuter, R., Tecza, M., et al. 2007, MNRAS, 378, 1229

Todorov, K., Luhman, K. L., \& Mcleod, K. K. 2010, ApJL, 714, L84

Tokovinin, A., Thomas, S., Sterzik, M., \& Udry, S. 2006, A\&A, 450, 681

Tokunaga, A. T., \& Vacca, W. D. 2005, PASP, 117, 421

Torres, C. A. O., da Silva, L., Quast, G. R., de la Reza, R., \& Jilinski, E. 2000, AJ, 120,1410

Torres, C. A. O., Quast, G. R., da Silva, L., et al. 2006, A\&A, 460, 695

Torres, C. A. O., Quast, G. R., de la Reza, R., et al. 2003, in Open Issues in Local Star Formation, ed. J. Lepine \& J. Gregorio-Hetem (Astrophysics and Space Science Library, Vol. 299; Dordrecht: Kluwer), 83

Torres, C. A. O., Quast, G. R., Melo, C. H. F., \& Sterzik, M. F. 2008, in Young Nearby Loose Associations, ed. B. Reipurth (San Francisco, CA: ASP), 757

Torres, G., Neuhäuser, R., \& Guenther, E. W. 2002, AJ, 123, 1701

Torres, G., \& Ribas, I. 2002, ApJ, 567, 1140

Udry, S., Bonfils, X., Delfosse, X., et al. 2007, A\&A, 469, L43

Vacca, W. D., Cushing, M. C., \& Rayner, J. T. 2003, PASP, 115, 389

van Altena, W. F., Lee, J. T., \& Hoffleit, E. D. 1995, The General Catalogue of Trigonometric Stellar Parallaxes (New Haven, CT: Yale University Observatory)

van Dam, M. A., Bouchez, A. H., Le Mignant, D., et al. 2006, PASP, 118, 310

van Dokkum, P. G., \& Conroy, C. 2010, Natur, 468, 940

van Leeuwen, F. 2007, A\&A, 474, 653

Vigan, A., Patience, J., Marois, C., et al. 2012, A\&A, 544, 9

Vorobyov, E. I., \& Basu, S. 2010, ApJL, 714, L133

Wahhaj, Z., Liu, M. C., Biller, B. A., et al. 2013a, ApJ, 779, 80

Wahhaj, Z., Liu, M. C., Nielsen, E. L., et al. 2013b, ApJ, 773, 179

Webb, R. A., Zuckerman, B., Platais, I., et al. 1999, ApJL, 512, L63

Weinberger, A. J., Anglada-Escudé, G., \& Boss, A. P. 2013, ApJ, 762, 118

West, A. A., Hawley, S. L., Bochanski, J. J., et al. 2008, AJ, 135, 785

Wizinowich, P., Acton, D. S., Shelton, C., et al. 2000, PASP, 112, 315

Wizinowich, P. L., Le Mignant, D., Bouchez, A. H., et al. 2006, PASP, 118, 297

Yee, J. C., \& Jensen, E. L. N. 2010, ApJ, 711, 303

Yelda, S., Lu, J. R., Ghez, A. M., et al. 2010, ApJ, 725, 331

Zacharias, N., Finch, C. T., Girard, T. M., et al. 2013, AJ, 145, 44

Zacharias, N., Monet, D. G., Levine, S. E., et al. 2005, yCat, 1297, 0

Zapatero Osorio, M. R., Bejar, V. J. S., Miles-Páez, P., et al. 2014, A\&A, 568, A6

Zuckerman, B., Song, I., \& Bessell, M. S. 2004, ApJL, 613, L65

Zuckerman, B., Song, I., \& Webb, R. A. 2001, ApJ, 559, 388 